\title{
Role of BRD4 and its target Ptp1b in Endothelial cells and in cardiovascular disease models
}

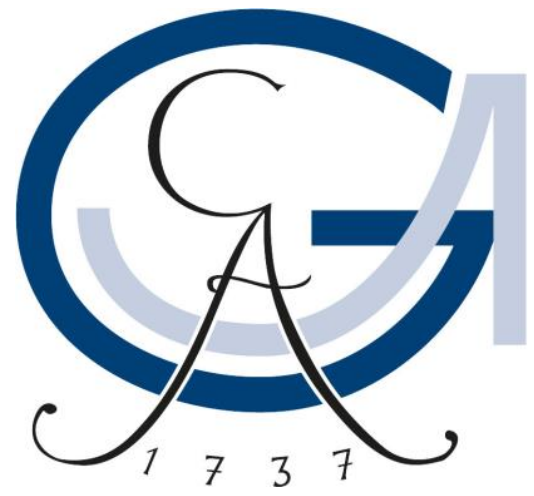

Dissertation

For the award of the degree

"Doctor of Philosophy (Ph.D.)"

at the Georg-August University Goettingen

Within the doctoral Programme GAUSS-Biology

of the Georg-August University School of Sciences (GAUSS)

Submitted by

Sidra Shahid

From Lahore, Pakistan

Goettingen 2019 


\section{Thesis Advisory Committee}

1. Prof. Dr. Ibrahim Adham Institute of Human Genetics, University Medical Centre Goettingen (UMG) Georg-August University Goettingen Germany.

2. Prof. Dr. Sigrid Hoyer-Fender

Department of Development Biology, Georg-August University Goettingen Germany.

\section{Dr. Krishna Pantakani}

Institute for Clinical Chemistry, University Medical Centre Goettingen (UMG) Georg-August University Goettingen Germany.

\section{Members of examination board}

Referee: $\quad$ Prof. Dr. Ibrahim Adham

Institute of Human Genetics, University Medical Centre Goettingen (UMG), Georg-August University Goettingen Germany.

Co-referee: Prof. Dr. Sigrid Hoyer-Fender

Department of Developmental Biology, Georg-August University Goettingen Germany.

\section{Other members of examination board}

1.

Prof. Dr. Uwe Groß

Institute for Medical Microbiology, University Medical Centre Goettingen.

2.

\section{Prof. Dr. Ahmed Mansouri}

Max Planck Institute for Biophysical Chemistry, Goettingen.

3.

$$
\text { Prof. Dr. Ralf Dressel }
$$

Institute for Cellular and Molecular Immunology, University Medical Centre Goettingen.

4.

$$
\text { PD, Dr. Michael Hoppert }
$$

Department of General Microbiology, Georg August University Goettingen.

Date of oral examination: 13.02 .2019 


\section{Declaration}

I hereby declare that the Ph.D. thesis entitled "Role of BRD4 and its target Ptp1b in Endothelial cells and in cardiovascular disease models" has been written independently, with no other sources than quoted, and has not been used or submitted in support of an application for another degree.

Sidra Shahid

17.01.2019 
Dedicated to my parents, family and friends for their love, affection, encouragement and support 


\section{Table of Contents}

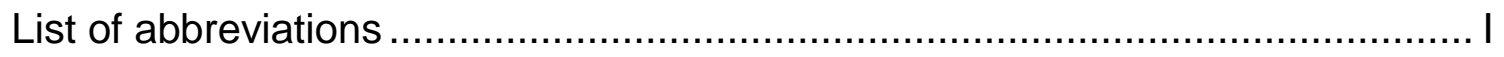

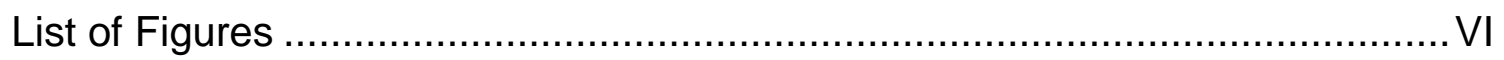

List of Tables..................................................................................

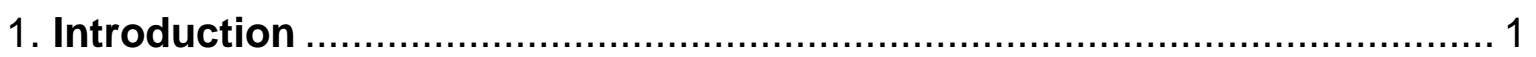

1.1 Modifiable risk factors in CVDs ......................................................... 2

1.1.1 Diabetes.................................................................................. 3

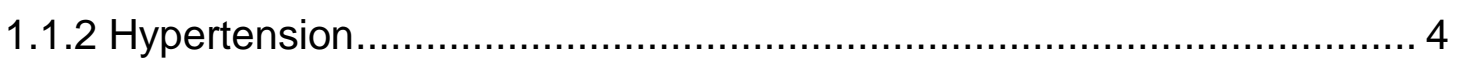

1.1.3 Elevated levels of Cholesterol...................................................... 4

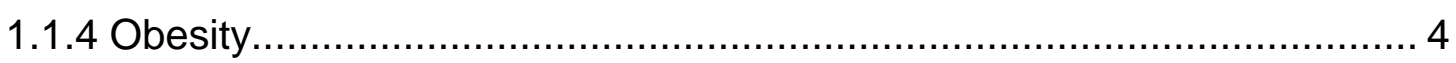

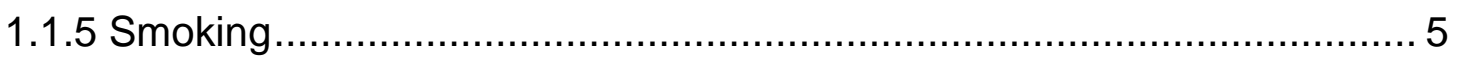

1.2 Non-modifiable Risk factors in CVDs ................................................ 5

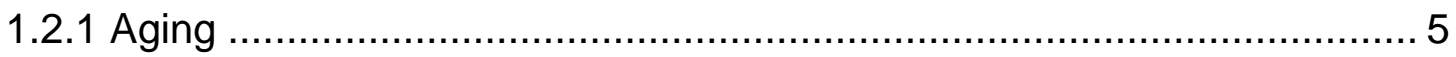

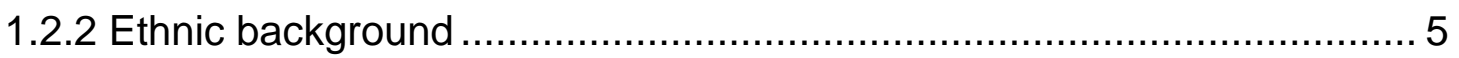

1.2.3 Family History/Genetic factor ................................................ 5

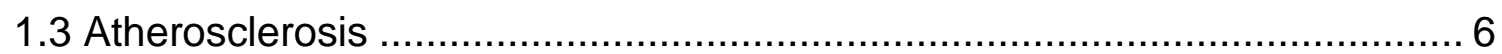

1.3.1 Stages of Atherosclerosis ............................................................ 6

1.3.2 Signalling Pathways and transcription factors involved in Atherosclerosis

1.3.3 Role of BRD4 in atherosclerosis .............................................. 10

1.4 Cardiac Hypertrophy ............................................................... 13

1.4.1 Signalling Pathways in Cardiac Hypertrophy .................................. 14

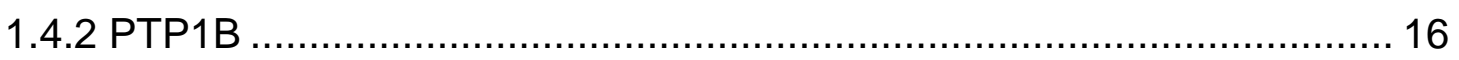

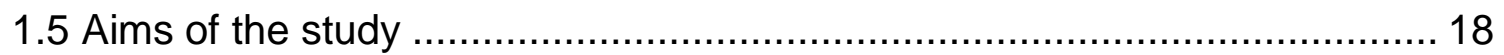

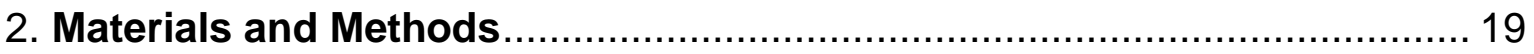

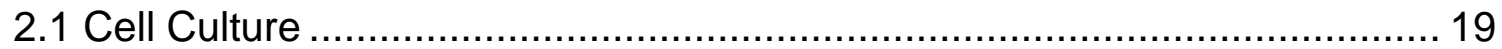

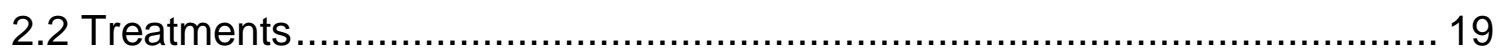

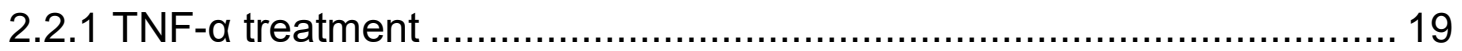

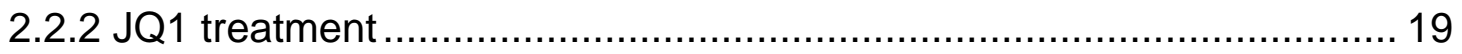

2.2.3 RVX208 treatment ..................................................................... 19

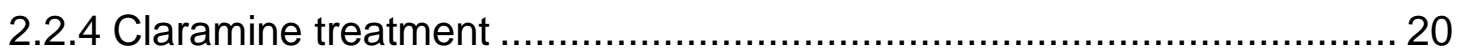

2.3 Immunofluorescence staining of HUVECs ........................................ 20

2.4 RNA expression analysis ............................................................ 20

2.4.1 RNA extraction from Cells/Tissues .......................................... 20 
2.4.3 Quantitative Real Time Polymerase Chain Reaction (qRT-PCR) ......... 21

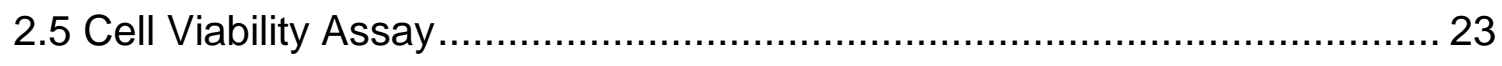

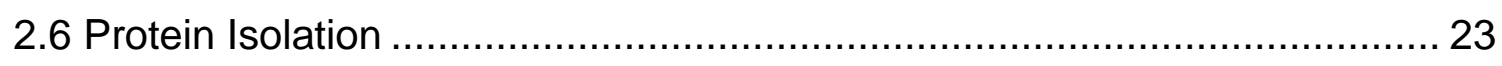

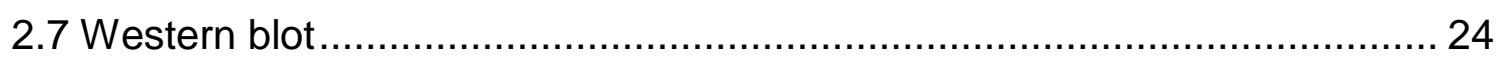

2.8 Trans-Endothelial/Epithelial Electrical Resistance (TEER) ....................... 24

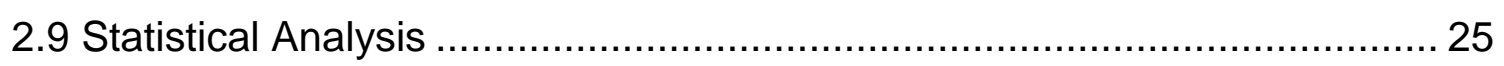

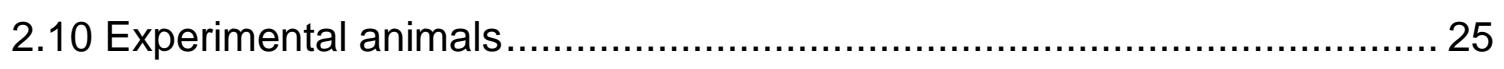

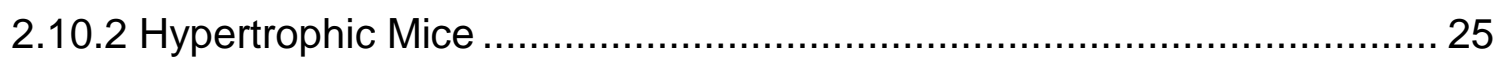

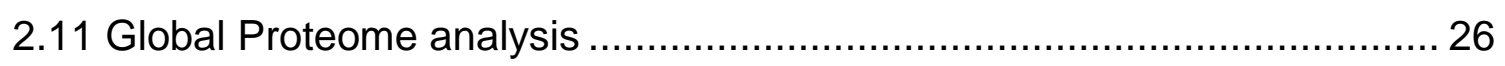

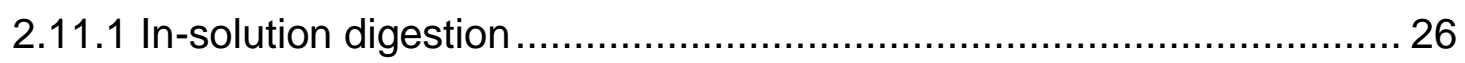

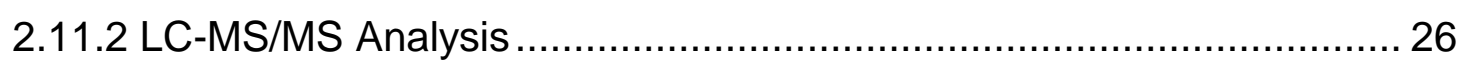

2.11.3 Bioinformatic analysis of mass spectrometry data ........................... 27

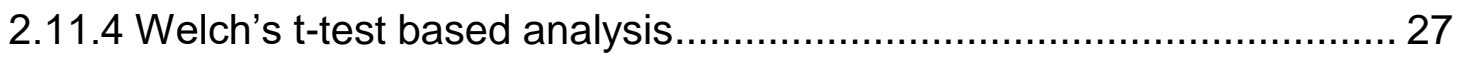

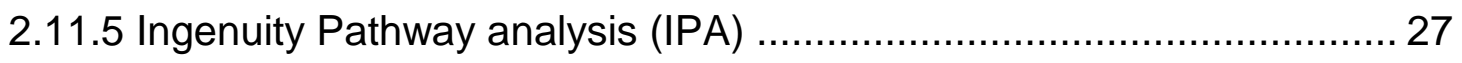

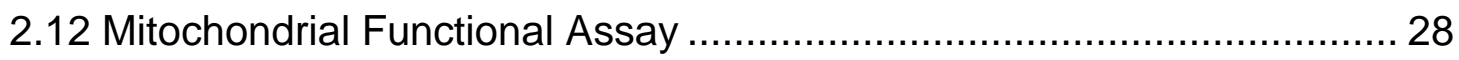

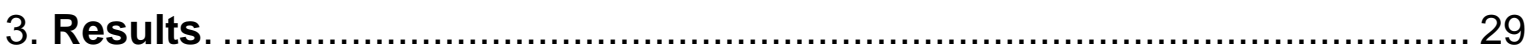

3.1 Establishment of in-vitro inflammatory model using HUVECs.................... 29

3.2 Role of BRD4 during inflammation........................................................ 30

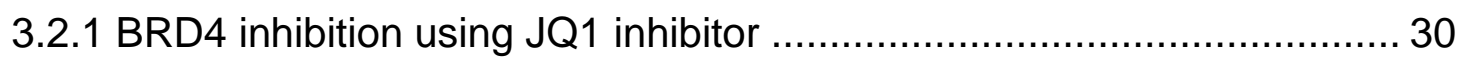

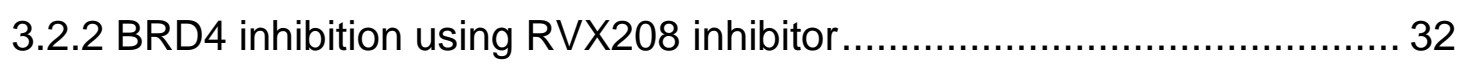

3.3 Elucidating the role of BRD4 isoforms in endothelial dysfunction and in

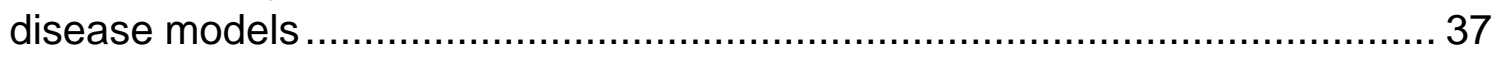

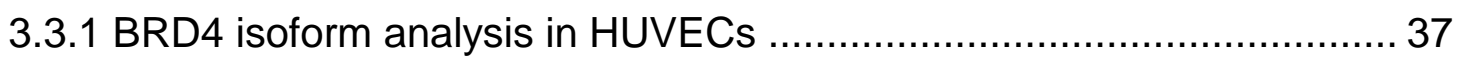

3.3.2 BRD4 isoforms expression analysis in CVDs models .......................... 40

3.4 Identifying the role of BRD4 and Midkine in monolayer integrity of

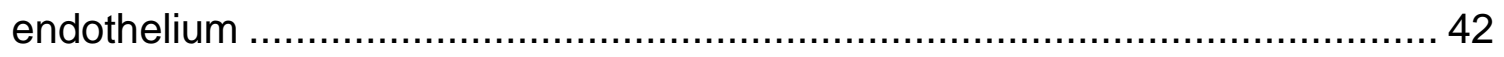

3.4.1 Effect of inflammatory phenotype on HUVECs monolayer integrity ...... 42

3.4.2 BRD4 inhibition protects HUVECs monolayer integrity ........................ 43

3.4.3 TNF- $\alpha$ induces the expression of Midkine ............................................ 45

3.4.4 BRD4 inhibition reduces the expression of Midkine ............................ 47

3.5 Expression of PTP1B increases in ECs during inflammation ...................... 49

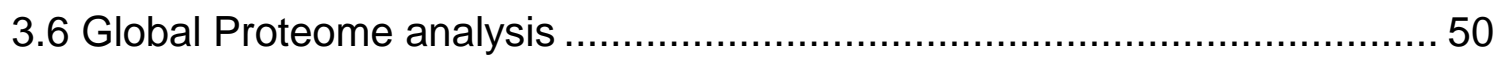

3.6.1 Statistical Analysis using Perseus Software ………......................... 53

3.6.2 Functional characterization of proteome data using STRING analysis . 67 
3.6.3 Ingenuity Pathway Analysis (IPA) for significantly differentiated proteins

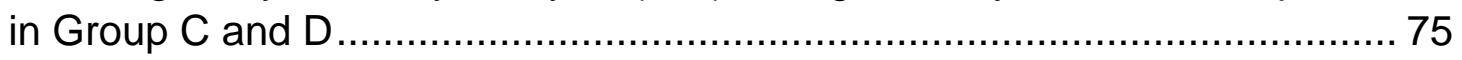

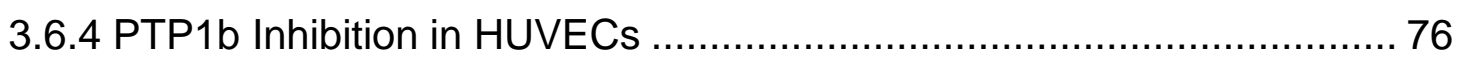

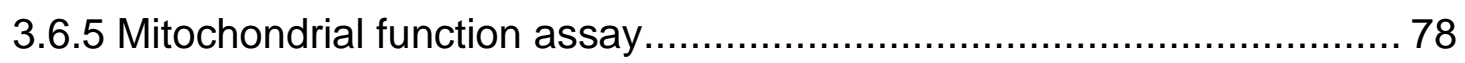

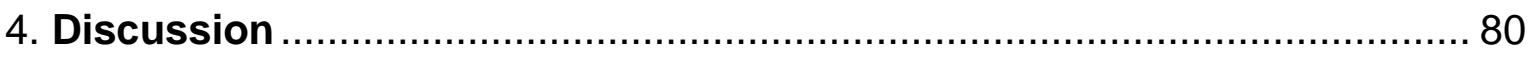

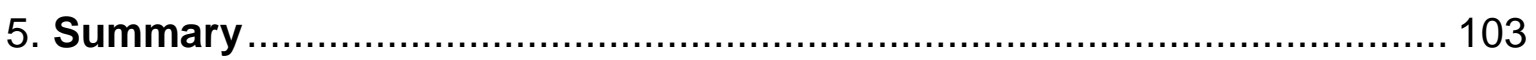

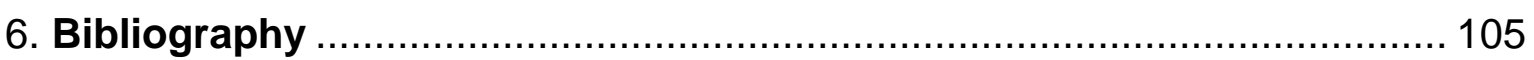

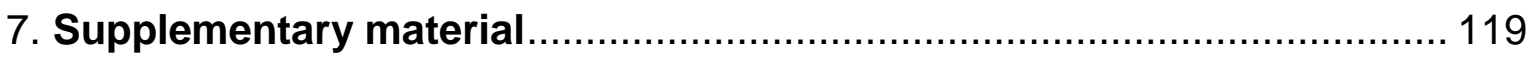

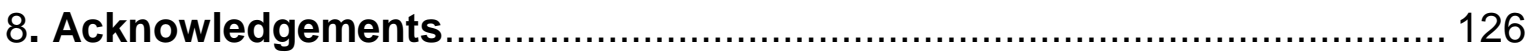

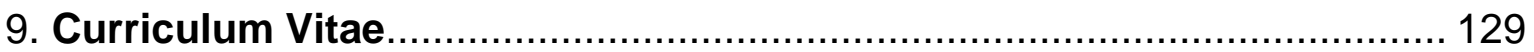




\section{List of abbreviations}

Ang-II

ANOVA

Apo-A1

ATP

BD1

BD2

BET

$\mathrm{BH} 4$

BRD4

BRD4-L

BRD4-S

BSA

$\mathrm{C} 2 \mathrm{C} 12$

Caco-2

Cam

CAs

CA-I

CA-II

Cal27

Cav-I

ChIP

ChIP-seq

CnA

$\mathrm{CnB}$

$\mathrm{CO}_{2}$

CSFR-1

CTD

CVDs

$\mathrm{Da}$

DAPI

DEPC
Angiotension II

Analysis of variance

Apolipoprotein-A1

Adenosine triphosphate

Bromodomain 1

Bromodomain 2

Bromodomain and extra terminal domain

(6R)-I-erythro-5,6,7,8-tetrahydrobiopterin

Bromodomain containing protein 4

Bromodomain containing protein 4 long isoform

Bromodomain containing protein 4 short isoform

Bovine serum albumin

Muscle myoblasts cell line

Human epithelial colorectal adenocarcinoma cells

Calmodulin

Carbonic anhydrases

Carbonic anhydrase-I

Carbonic anhydrase-II

Oral carcinoma cell line

Caveolin I

Chromatin immunoprecipitation

Chromatin immunoprecipitation with massively parallel

DNA sequencing

Calcineurin $A$

Calcineurin B

Carbon dioxide

Colony stimulating factor 1 receptor

Carboxy-terminal domain

Cardiovascular diseases

Dalton

4 ,6-Diamidino-2Phenylindole, Dihydrochloride

Diethylpyrocarbonate 


$\begin{array}{ll}\text { DIA } & \text { Data independent acquisition } \\ \text { DMEM } & \text { Dulbecco's modified eagle medium } \\ \text { DMSO } & \text { Dimethyl sulfoxide } \\ \text { dNTPs } & \text { deoxyribonucleotide triphosphate } \\ \text { DPBS } & \text { Dulbecco's phosphate-buffered saline } \\ \text { DTT } & \text { Dithiothreitol } \\ \text { ECM } & \text { Extracellular matrix } \\ \text { ECs } & \text { Endothelial cells } \\ \text { EDTA } & \text { Ethylenediaminetetraacetic acid } \\ \text { EGTA } & \text { Egtazic acid } \\ \text { Endo.Ptp1b.KO } & \text { Mice with endothelial-specific deletion of PTP1B } \\ \text { Endo.Ptp1b.WT } & \text { Wild Type mice } \\ \text { eNO } & \text { Endothelial specific nitric oxide } \\ \text { eNOS } & \text { Endothelial specific nitric oxide synthase } \\ \text { ER } & \text { Endoplasmic reticulum } \\ \text { ET } & \text { Extra terminal domain } \\ \text { et al., } & \text { "and others." } \\ \text { ETC } & \text { Electron transport chain } \\ \text { ET-I } & \text { Endothelin-1 } \\ \text { EVOM2 } & \text { Epithelial voltammeter } \\ \text { FCS } & \text { Foetal calf serum } \\ \text { FDR } & \text { False discovery rate } \\ \text { FGF } & \text { Fibroblast growth factors } \\ \text { FHL1 } & \text { Four and a half LIM domains 1 } \\ \text { FWHM } & \text { Full width at half maximum } \\ \text { GAPDH } & \text { Glyceraldehyde 3-phosphate dehydrogenase } \\ \text { GJA1 } & \text { Gap junction alpha-1 protein } \\ \text { GLUT4 } & \text { Glucose transporter } \\ \text { GO } & \text { Gene ontology } \\ \text { GPCRs } & \\ \text { Gpx } & \text { Gonstriction, used as an experimental control } \\ \text { KO-SHAM } & \end{array}$


KO-TAC

H3K14

H4K12

H4K5

HATs

HDACs

HDL

HRP-conjugated

$\mathrm{hr}$

hrs

HUVECS

IAA

ICAM-1

IGF

IGFR

IKB- $\alpha$

IKK

IL1

IL1 $\beta$

IL6

IL8

IPA

IRAK1

JAK

JQ1

$\mathrm{kDa}$

LC-MS/MS

LDL

LDL-Cholesterol

LPS

MAPKs
Ptp1b knockout mice with transverse aortic constriction

Histone 3 lysine 14

Histone 4 lysine 12

Histone 4 lysine 5

Histone acetyltransferases

Histone deacetylases

High-density lipoprotein

Horseradish peroxidase-conjugated

Hour

Hours

Human Umbilical cord derived vascular endothelial cells lodoacetamide

Intercellular adhesion molecule 1

Insulin growth factors

Insulin growth factor receptor

NF-kB inhibitor

IKB-Kinase

Interleukin-1

Interleukin $1 \beta$

Interleukin-6

Interleukin-8

Ingenuity pathway analysis

Interleukin receptor associated kinase 1

Janus-Kinase

(6S)-4-(4-chlorophenyl)-2,3,9-trimethyl-6H-thieno[3,2-

f][1,2,4]triazolo[4,3-a][1,4]diazepine-6-acetic acid, 1,1-

dimethylethyl ester

Kilo Dalton

Liquid chromatography-tandem mass spectrometry

Low-density lipoprotein

Low-density lipoprotein cholesterol

Lipopolysaccharides

Mitogen activated protein kinases 


$\begin{array}{ll}\text { MHCs } & \text { Myosin heavy chains } \\ \text { MI } & \text { Myocardial Infarction } \\ \text { mins } & \text { minutes } \\ \text { MLCs } & \text { Myosin light chains } \\ \text { mM } & \text { Mili molar } \\ \text { M-MLV-RT } & \text { Moloney murine leukemia virus reverse transcriptase } \\ \text { mTOR } & \text { Mammalian target of rapamycin } \\ \text { MyD88 } & \text { Myeloid differentiation primary response 88 } \\ \text { Na2EDTA } & \text { Disodium ethylenediaminetetraacetic acid } \\ \text { Na3VO4 } & \text { Sodium orthovanadate } \\ \text { NFAT } & \text { Nuclear factor of activated T cells } \\ \text { NF-KB } & \text { Nuclear transcription factor kB } \\ \text { nM } & \text { Nano molar } \\ \text { NO } & \text { Nitric oxide } \\ \text { p65 } & \text { RelA subunit of NF-kB } \\ \text { PAK-1 } & \text { p21-activated kinase-1 } \\ \text { PCA } & \text { Principal component analysis } \\ \text { PCR } & \text { Polymerase chain reaction } \\ \text { PDGFR } & \text { Platelet derived growth factor receptor } \\ \text { PFA } & \text { Paraformaldehyde } \\ \text { PI3K } & \text { Phosphoinositol-3-kinase } \\ \text { PMSF } & \text { Phenylmethylsulfonyl fluoride } \\ \text { P-TEFB } & \text { Positive transcription elongation factor } \\ \text { PTP1B } & \text { Protein tyrosine phosphatase 1B } \\ \text { Ptp1b-KO } & \text { Mice with PTP1B deficiency } \\ \text { PTPs } & \text { Protein tyrosine phosphatases } \\ \text { qRT-PCR } & \text { Quantitative real time polymerase chain reaction } \\ \text { RNA Pol-II } & \text { RNA polymerase II } \\ \text { ROS } & \text { Reactive oxygen species } \\ \text { RT } & \text { Room temperature } \\ \text { RTKs } & \text { RVX-208 } \\ \text { RVydroxyethoxy)-3,5-dimethylphenyl]-5,7- } \\ \end{array}$


Sodium dodecyl sulfate

SDS-PAGE

Sodium dodecyl sulfate polyacrylamide gel

electrophoresis

SELE

E-Selectin

SEM

Standard error of mean

SEs

Super enhancers

shRNA

Short hairpin RNA

SMCs

Smooth muscle cells

STAT

Signal transducer activator of transcription

STRING

Search Tool for the Retrieval of Interacting

Genes/Proteins

SWATH

Sequential Window Acquisition of all Theoretical

fragment ion spectra

TAC

Transverse aortic constriction

TBST

Tris-buffered saline with tween

TEER

Trans-endothelial/epithelial electrical resistance

TGF $\beta$

Transforming growth factor $\beta$

Tie2.ERT2.Cre

Mice expressing a fusion protein of $C r e$ recombinase-

estrogen receptor under the control of endothelial receptor tyrosine kinase promoter

TLR2TL-2

Toll like receptor-2

TLR4/TL-4

Toll like receptor-4

TNF- $\alpha$

Tumor necrosis factor $\alpha$

TNFR

Tumor necrosis factor receptor

VCAM-I

Vascular cell adhesion molecule 1

VEGFR

Vascular endothelial growth factor receptor

WT

WT-SHAM

Wild type

WT-TAC

Wild type mice without transverse aortic constriction

$\mu \mathrm{g}$

Wild type mice with transverse aortic constriction

$\mu \mathrm{l}$

Micro gram

Micro liter

$\mu \mathrm{M}$

Micro molar 


\section{List of Figures}

Figure 1.1 Risk factors in CVDs 3

$\begin{array}{lll}\text { Figure } 1.2 & \text { Stages of Atherosclerosis } & 7\end{array}$

Figure 1.3 NF-kB Signalling pathway 9

Figure 1.4 Binding of BRD4 on chromatin and acting as HAT and Kinase 10

Figure 1.5 Human BRD4 protein isoforms structure 11

Figure 1.6 Redistribution of BRD4 on chromatin during Inflammatory 12 stimulus

Figure 1.7 The structure of a normal and cardiac hypertrophy heart 13

Figure 1.8 Signalling pathways in Cardiac hypertrophy 15

Figure 1.9 Domain architecture of PTP1B 16

Figure 3.1 Establishment of inflammatory phenotype in HUVECs 29

Figure 3.2 BRD4 inhibition using small molecule inhibitor JQ1 31

Figure 3.3 Optimization of RVX208 treatment for inhibition of BRD4 33

Figure 3.4 BRD4 inhibition using RVX208 and JQ1 35

Figure 3.5 Expression analysis of BRD4 isoforms in HUVECs 38

Figure 3.6 Expression analysis of BRD4 protein in HUVECs 39

Figure 3.7 Expression of BRD4 isoforms in mice hearts 41

Figure 3.8 Disruption of monolayer integrity in HUVECs 43

Figure 3.9 BRD4 inhibition, protects monolayer integrity in HUVECs 44

Figure 3.10 Induction of MIDKINE and p38MAPK expression in HUVECs 45

Figure 3.11 Midkine expression in HUVEC monolayer during TNF- $\alpha \quad 46$ treatment

Figure 3.12 Midkine inhibition in HUVECs with BRD4 inhibitors 47-48

Figure 3.13 Induction of PTP1B expression in HUVECs 50

Figure 3.14 Schematic illustration of strategy for global proteome analysis 51

Figure 3.15 Global proteome analysis 52

Figure 3.16 Statistical summary of Global proteome analysis 54

$\begin{array}{lll}\text { Figure 3.17 Distribution of identified proteins in global proteome analysis } & 67\end{array}$

Figure 3.18 Characterization for Group A, WT-SHAM vs KO-SHAM 69 proteome

Figure 3.19 Characterization for Group B, WT-SHAM vs WT-TAC 70 
proteome.

Figure 3.20 Characterization for Group C, KO-SHAM vs KO-TAC proteome

Figure 3.21 Characterization for Group D, WT-TAC vs KO-TAC proteome

Figure 3.22 Ingenuity pathway analysis (IPA) of KO-TAC vs WT-TAC proteins

Figure 3.23 PTP1B inhibition in HUVECs

Figure 3.24 Evaluation of mitochondrial toxicity in in-vitro inflammatory model

Figure 4.1 Factors involved in ECs activation, inflammation, and in atherosclerotic plaque development

Figure 4.2 LPS mediated NF-KB activation

Figure 4.3 TNF- $\alpha$ mediated NF-KB activation contributes in ECs dysfunction

Figure 4.4 BRD4 genome binding in absence and presence of TNF- $\alpha$ 85

Figure 4.5 Summary of inflammatory response in ECs 89

Figure 4.6 BRD4 inhibition reduces the expression of pro-inflammatory markers

Figure 4.7 PTP1B target receptors

Figure 4.8 Mitochondrial dysfunction in CVDs 95

Figure 4.9 IPA analysis of WT-TAC proteins compared to WT-SHAM 96 proteins

Figure 4.10 Summary of global proteome comparison in WT-TAC and 101 KO-SHAM

Figure 4.11 Conclusion: TNF- $\alpha$, BRD4 and PTP1B function on ECs and CVDs

Figure S1 Full view of STRING analysis of Group A, WT-SHAM vs KO- 119 SHAM proteome

Figure S2 Full view of STRING analysis of Group B, WT-SHAM vs WT- 120 TAC proteome (up-regulated).

Figure S3 Full view of STRING analysis of Group B, WT-SHAM vs WT121 TAC proteome (down-regulated). 
Figure S4 Full view of STRING analysis of Group C, KO-SHAM vs KO- 122 TAC proteome (up-regulated).

Figure S5 Full view of STRING analysis of Group C, KO-SHAM vs KO- 123 TAC proteome (down-regulated).

Figure S6 Full view of STRING analysis of Group D, WT-TAC vs KO-TAC 124 proteome (up-regulated).

Figure S7 Full view of STRING analysis of Group D, WT-TAC vs KO-TAC 125 proteome (down-regulated).

\section{List of Tables}

Table 2.1 Human mRNA expression primer list 22

Table 2.2 Mouse mRNA expression primer list 23

Table 3.1 Group A: Up-regulated Proteins in KO-SHAM vs WT- 54

Table 3.2 Group A: Down-regulated Proteins in KO-SHAM vs WT- 55 SHAM

Table 3.3 Group B: Up-regulated Proteins in WT-TAC vs WT-

SHAM

Table 3.4 Group B: Down-regulated Proteins in WT-TAC vs WT-

SHAM

Table 3.5 Group C: Up-regulated Proteins in KO-TAC vs KO-SHAM

Table 3.6 Group C: Down-regulated Proteins KO-TAC vs KO-SHAM

Table 3.7 Group D: Up-regulated Proteins in KO-TAC vs WT-TAC

Table 3.8 Group D: Down-regulated Proteins in KO-TAC vs WT-TAC

Table 4.1 Comparison of our proteomic results with different

published LC-MS/MS based proteomic studies on TAC animals 


\section{Introduction}

Cardiovascular diseases (CVDs), relating to heart and circulatory system, are among the leading causes of death worldwide. In developing countries, $31 \%$ of mortality rate is due to CVDs, and it has been estimated that it will become the major cause of deaths by 2020 (Stewart et al., 2017). A wide spectrum of cardiac and vascular pathologies such as cardiac fibrosis, reduced angiogenesis after myocardial infarction (MI), atherosclerosis and cardiac hypertrophy are some important forms of CVDs (World Health organization report 20142017) (Banquet et al., 2011; Carmeliet, 2005).

- Cardiac fibrosis is referred as abnormal thickening of cardiac muscles with deposition of extracellular matrix (ECM), which reduces the mechanical function of heart (Tian et al., 2017). In normal cardiac function, cardiac fibroblasts maintain the ECM secretion like Collagen I and III in cardiac tissues (Nicoletti and Michel, 1999). This homeostatic balance of ECM is necessary to maintain mechanical force and electrical conductance in heart tissue (Travers et al., 2016). Hypertension, hyperglycemia and ischemia effect these fibroblasts leading to transforming them into myofibroblasts which secrete altered ECM proteins, mainly high amount of Collagen I (Nicoletti and Michel, 1999). This altered ECM reduces the cardiac tissue efficiency and promotes fibrosis, which leads to cardiac failure (Nicoletti and Michel, 1999; Travers et al., 2016).

- Myocardial infarction (MI), also known as heart attack, occurs when there is insufficient blood supply to cardiac tissues (ischemia) (Kobayashi et al., 2017). Angiogenesis, formation of new capillaries is a response of cardiac tissue to compensate the ischemic condition (Hueso et al., 2017; Kobayashi et al., 2017). Reduced angiogenesis is a common pathological condition in CVDs (Kobayashi et al., 2017). Prolonged ischemic conditions can lead to metabolic imbalance in ischemic tissues, which leads to cardiac failure (Hueso et al., 2017). Therapeutic studies focused on improving cardiac angiogenesis after $\mathrm{Ml}$ shows that it results in reduced risk of cardiac failure (Hueso et al., 2017; Kobayashi et al., 2017).

- Atherosclerosis is another common pathological condition associated with prognosis of multiple CVDs. Atherosclerosis is a disease, in which plaques develop inside the arterial walls (Verstraete, 1990; Zampetaki et al., 2010). Disturbed blood flow, hypertension and high cholesterol levels in blood exerts stress on arterial wall, 
which leads to endothelial cells (ECs) dysfunction (Kwak et al., 2014; Zampetaki et al., 2010). ECs dysfunction leads to atherosclerotic plaque development (Kwak et al., 2014). Unstable plaques in atherosclerosis can rupture and activate the immune system, which develops a thrombus (Kwak et al., 2014).

- Cardiac hypertrophy is defined as abnormal enlargement of cardiac muscles due to increased size of cardiomyocytes and deposition of ECM as a result of prolonged cardiac fibrosis (Hara et al., 2017). Cardiac chambers, specifically left ventricle, become enlarge due to deposition of excess ECM, which leads to cardiomyocyte death (Samak et al., 2016). Hypertrophic tissues become stiff and reduce the blood pumping efficiency of heart, which can lead to cardiac failure (Heineke and Molkentin, 2006; Samak et al., 2016).

Among several forms of CVDs, atherosclerosis and cardiac hypertrophy are the focus of this thesis and will be discussed further in detail. Multiple environmental and biological factors contribute in the development of CVDs. Major risk factors for the development of atherosclerosis and cardiac hypertrophy can be divided into two categories: 1 . Modifiable risk factors (diabetes, hypertension, elevated levels of cholesterol, obesity, and smoking) and 2. Non-modifiable risk factors (Aging, ethnicity, family history/genetic and gender).

\subsection{Modifiable risk factors in CVDs}

Modifiable risk factors include those factors, which are preventable and can reduce the risk of developing atherosclerosis or cardiac hypertrophy. Major modifiable risk factors are diabetes, hypertension, elevated levels of cholesterol in blood, obesity and smoking (Fig. 1.1) (Deaton et al., 2011). 


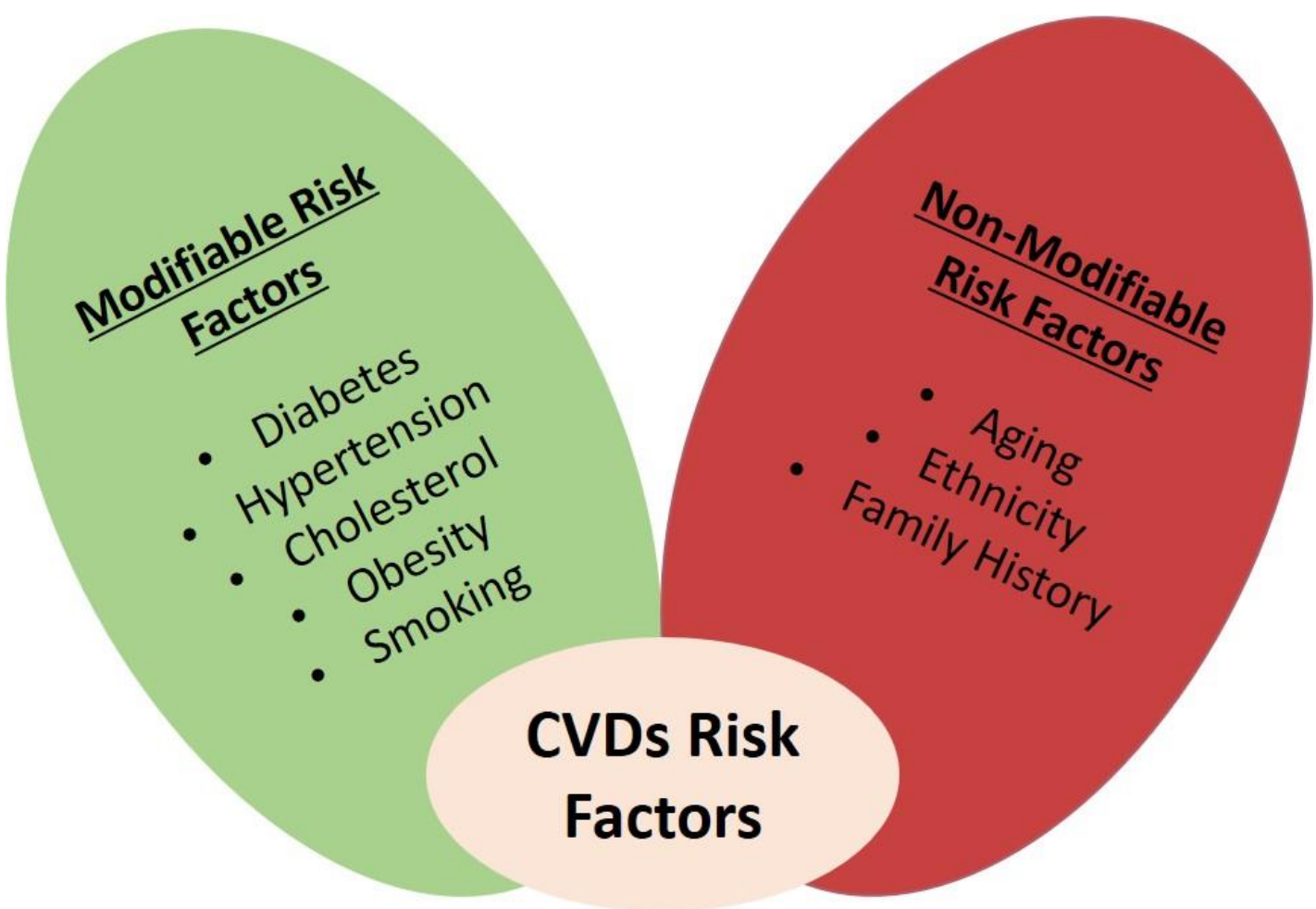

Figure 1.1. Risk Factors in CVDs. Modifiable risk factors (green) are those that can be controlled or improved. These include diabetes, hypertension, cholesterol, obesity and smoking. Non-modifiable factors (red) are those that cannot be altered like age, ethnicity and family history (Genetic contribution) of disease.

\subsubsection{Diabetes}

Diabetes, also known as hyperglycemia (high blood sugar level) is classified as a modifiable risk factor. Once developed, it cannot be cured completely. However controlling blood sugar levels can reduce the risk of CVDs development (Leon and Maddox, 2015). Hyperglycemia leads to glycation of cholesterol molecule, which enhances its oxidation thereby leads to ECs dysfunction and promotes CVDs (Rosiek and Leksowski, 2016). The endothelium (ECs) is not only important to maintain homeostatic balance between blood and tissues (Fig. 1.2A) (Hadi et al., 2005; Messner and Bernhard, 2014), but also for nonadhesive, anticoagulant, anti-hypertrophic and anti-inflammatory functions of endothelium dependent vasodilation (Hadi et al., 2005; Michaelis, 2014). Intriguingly, Insulin resistance and hyperglycemia contributes to the development of atherosclerosis and ECs dysfunction via reducing the production of endothelial Nitric Oxide Synthase (eNOS) and by enhanced production of Reactive Oxygen Species (ROS) (Leon and Maddox, 2015). The low levels of eNOS production leads to reduced ECs-dependent-vasodilation. While elevated levels 
of ROS cause cell death and injury in ECs, which leads to inflammatory response and the development of CVDs (Leon and Maddox, 2015).

\subsubsection{Hypertension}

Hypertension, or high blood pressure, is developed by environmental and genetic factors and contributes significantly to mortality associated with CVDs. It is known that persons with hypertension have $50 \%$ more chances to develop heart diseases compared to nonhypertensive person (Huq et al., 2018). Hypertension increases the risk of ECs dysfunction and atherosclerosis probably by increasing ROS production and various vasoconstrictors such as endothelin-1 (ET-I) and Angiotension II (Ang-II), production (Higashi et al., 2012; Rosiek and Leksowski, 2016). Elevated levels of ROS and vasoconstrictors disrupt ECs dependent vasodilation and leads to the development of CVDs (Bleakley et al., 2015; Higashi et al., 2012).

\subsubsection{Elevated levels of Cholesterol}

Cholesterol is classified as one of the major contributing factor in CVDs development (Rosiek and Leksowski, 2016). High levels of low-density lipoprotein cholesterol (LDLCholesterol) and triglycerides, collectively known as dyslipidemia, impairs ECs function by perturbing the eNOS signalling pathway (Dart and Chin-Dusting, 1999; Kim et al., 2012). Nitric Oxide (NO), a vasodilatory gas, derived from eNOS signalling pathway, is responsible for maintaining anti-apoptotic and anti-proliferative environment in blood vessels (Sessa, 2004). Dyslipidemia is also known to stimulate leukocyte adhesion to ECs, therefore, contributes in atherosclerosis development (Dart and Chin-Dusting, 1999). LDLCholesterol also contributes in progression of atherosclerotic lesions formation and is a dominating contributing molecule in lipid core of atherosclerotic lesions (Hadi et al., 2005; Rosiek and Leksowski, 2016).

\subsubsection{Obesity}

Obesity is a multifactorial disorder, in which both genetic and environmental factors are involved (Wang and Nakayama, 2010). Obesity indirectly increases the risk for CVDs development as a result of the development of diabetes, hypertension, and high levels of cholesterol in blood (Kachur et al., 2017). It has been reported that obese children have impaired contractile function and develop left ventricular hypertrophy (Jing et al., 2016). Obesity leads to disruption in lipid metabolism and increases ECs dysfunction due to 
increased inflammation (Lovren et al., 2015). These attributes of obesity make a person more prone to develop atherosclerosis as compared to non-obese persons (Lovren et al., 2015).

\subsubsection{Smoking}

Smoking is directly linked to multiple diseases like lung cancer, ischemia, atherosclerosis, endothelial dysfunction, hypoxemia, and thrombosis (Hadi et al., 2005). Smoking impairs the balance between endothelium specific vasoconstrictor and vasodilatory chemical production (Messner and Bernhard, 2014; Michael Pittilo, 2000). Smoking has been also reported as a contributing factor in lipid profile alteration and to increase the expression of adhesion molecules on ECs and reduce eNO production, which leads to endothelial dysfunction and atherosclerosis development (Messner and Bernhard, 2014).

\subsection{Non-modifiable Risk factors in CVDs}

Non-modifiable risk factors are those, which cannot be controlled or modified. These include Aging, ethnic background and family history.

\subsubsection{Aging}

Age is an independent risk factor that increases the risk of CVDs development in together with other risk factors such as diabetes, hypertension and high cholesterol levels (Dhingra and Vasan, 2011). Advanced age is associated with cellular senescence of ECs, loss of blood vessels elasticity (Hadi et al., 2005; Stewart et al., 2017) higher ROS production, which results in endothelial dysfunction and loss of ECs vasodilation ability (Higashi et al., 2012; Matz and Andriantsitohaina, 2003).

\subsubsection{Ethnic background}

It has been reported that South Asian population has higher risk of CVDs development as compared to European population. This is mainly due to life style differences, which lead to the development of diabetes, high LDL-Cholesterol levels and hypertension (Chaturvedi, 2003). Mexicans and African Americans are also reported to have higher risk of CVDs development (Chaturvedi, 2003; Forouhi and Sattar, 2006).

\subsubsection{Family History/Genetic factor}

Linkage analysis and genetic association studies have been one of a successful approach in determining CVDs development (Kathiresan and Srivastava, 2012). Risk of developing 
CVDs increases if first-degree and second-degree relatives are affected (Imes and Lewis, 2014). Risk of atherosclerosis development increases up to 3-fold if parents are atherosclerotic (Kathiresan and Srivastava, 2012). There is a $40 \%$ higher chance of CVDs development if a sibling is affected, while children of CVDs patients have $75 \%$ higher chance of developing CVDs (Imes and Lewis, 2014; Kolber and Scrimshaw, 12014).

Taken together, all the above-mentioned factors independently and synergistically contribute in the development of CVDs, including atherosclerosis and cardiac hypertrophy (Hadi et al., 2005; Rosiek and Leksowski, 2016).

\subsection{Atherosclerosis}

Atherosclerosis refers to the thickening of arterial wall, which occurs due to the accumulation of lipids and fibrous materials in major arteries. Mostly the branched areas, which are exposed to disturbed blood flow are more prone to plaque development (Chien, 2008; Zampetaki et al., 2010). There are three main layers in artery (Fig. 1.2A). The tunica intima is the inner most layer that is lined by monolayer of ECs. The middle layer, tunica media, mainly consists of smooth muscle cells (SMCs) that are embedded in ECM. The outer most layer, adventitia, contains fibroblasts, micro vessels, mast cells and nerve endings (Fig. 1.2A) (Chien, 2008; Libby, 2000; Zampetaki et al., 2010). The accumulation of lipids and fibrous material occurs mostly at branched areas, which are exposed to disturbed blood flow and ultimately leads to activation of pro-inflammatory signalling pathways (Chien, 2008). Lipid accumulation is also associated with higher apoptotic activity of ECS, reduced eNOS production, increased ROS production and responsible for increased permeability of ECs, which eventually leads to endothelial dysfunction and atherosclerosis (Zampetaki et al., 2010). Atherosclerotic lesion development starts with accumulation of monocytes into intima due to endothelial dysfunction.

\subsubsection{Stages of Atherosclerosis}

Atherosclerosis can be divided into following stages

1. Endothelial dysfunction

2. Migration and maturation of monocytes

3. Plaque formation

4. Thrombosis 

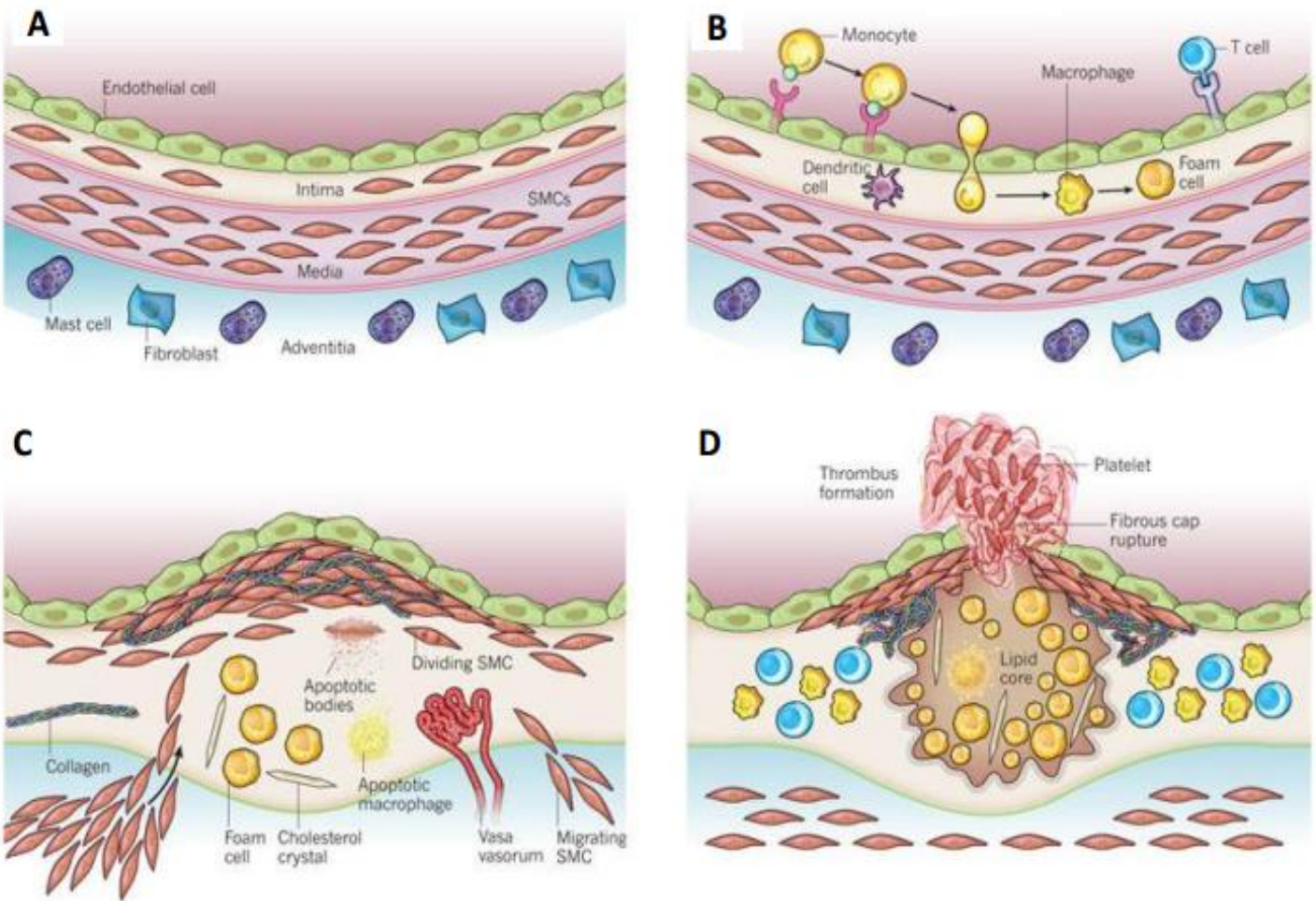

Figure 1.2. Stages of Atherosclerosis. A. The innermost layer of artery is lined by ECs, which maintain the homeostatic balance between blood and artery. Middle layer consists mainly of SMCs, while the outer most layer contains nerve cell endings, fibroblast cells and mast cells. B. Inflammatory response leads to activation of ECs, which primes binding of monocytes to ECs, and their migration into intima. Inside intima, monocytes mature into macrophages, take up lipid molecules, and form foam cells. C. SMCs along with collagen fibers migrate to intima and make thick fibrous cap. Lipid molecules accumulate in centre of the plaque and form lipid core. D. Plaque cap ruptures due to disturbed blood flow, which triggers the immune response, and a thrombus is formed. Thrombus can block the artery partially or completely. (Figure published by Libby et al., 2011).

\subsubsection{Endothelial Dysfunction}

Endothelium is important to maintain homeostatic balance between blood and tissues (Fig. 1.2A) (Chien, 2008; Zampetaki et al., 2010). Different environmental factors and signalling pathways are involved in protection and activation of ECs. Increased levels of homocysteine, LDL, micro-organisms, smoking and diabetes are considered as triggering factors of ECs dysfunction (Hadi et al., 2005; Higashi et al., 2012; Hueso et al., 2017; Messner and Bernhard, 2014). Cytokines like interleukin- $1 \beta$ and tumor necrosis factor $\alpha$ (TNF- $\alpha$ ) are responsible for the initiation of ECs dysfunction by activating nuclear transcription factor $\mathrm{KB}$ (NF-KB) and protein kinase $\mathrm{C}$ pathways (Brown et al., 2014; Xiao et al., 2014). NF-KB is a key regulator of inflammation during atherosclerosis (Brown et al., 2014). Activation of NF-KB leads to translocation of NF-KB from cytoplasm to the nucleus, which initiates transcription of downstream target genes including cytokines, chemokines and adhesion molecules like E-Selectin (SELE) and Vascular Cell Adhesion Molecule 1 
(VCAM-1) (Gerhardt and Ley, 2015). ECs express adhesion molecules on their surface, which starts interacting with different blood cells like monocytes.

\subsubsection{Migration and maturation of Monocytes}

Migration of monocytes into intima is a multistep process that involves capturing of monocytes on activated ECs, slow rolling on the surface of ECs, firm binding to ECs and then migration into intima (Gerhardt and Ley, 2015). SELE mediates the capturing and slow rolling of monocyte and T-cells on ECs, while VCAM-1 is responsible for firm adhesion of leukocytes on ECs, which facilitates its migration into intima (Fig. 1.2B) (Gerhardt and Ley, 2015; Libby, 2000). Migrated monocytes mature into macrophages, which fuse with lipoproteins to make foam cells (Gerhardt and Ley, 2015; Libby, 2000).

\subsubsection{Plaque Formation}

Foam cells accumulate to make a lipid core of lesion. SMCs migrate from tunica media to intima and accumulate in lesions along with cellular and ECM like collagen, proteoglycans, and elastin and form thick fibrous cap (Gerhardt and Ley, 2015; Libby, 2000; Xiao et al., 2014). The lesion protected by this thick fibrous cap is referred as atheroma or atherosclerotic plaque. Central lipid core of plaque, known as necrotic core, consists mainly of lipid molecules and cholesterol crystals (Fig. 1.2C) (Libby, 2000).

\subsubsection{Thrombosis}

Atherosclerotic plaques are of two types stable and unstable, based on their composition (Falk, 1992). Stable plaques are rich in ECM and SMCs and have thick stable cap. While unstable plaques are rich in soft extracellular lipids and highly infiltrated with foam cells leading to be more prone to rupture (Falk, 1992). Fibrous cap of unstable plaques ruptures due to physical abrasion from blood flow and activates acute thrombosis response that can lead to blood coagulation and thrombus formation (Fig. 1.2D). Thrombus can extend ,restrict the lumen of the artery, and can cause further complications like angina, myocardial infarction, dysrhythmia and sudden cardiac arrest (Libby, 2000). Atherosclerosis reduces the normal blood flow, which can cause cardiac arrest and death.

\subsubsection{Signalling Pathways and transcription factors involved in Atherosclerosis}

Atherosclerosis is categorized as an inflammatory disease. Inflammation is the important step in initiation and progression of plaque formation (Conti and Shaik-Dasthagirisaeb, 2015). Multiple signalling pathways have been identified as regulators of inflammation such as mitogen activated protein kinases (MAPKs), signal transducer activator of transcription 
(STAT), Janus-Kinase (JAK) and NF-KB (Montecucco et al., 2012; von der Thusen et al., 2003). Among these signalling pathways, NF-kB is the main signalling pathway of inflammation (Hajra et al., 2000; Xiao et al., 2014).

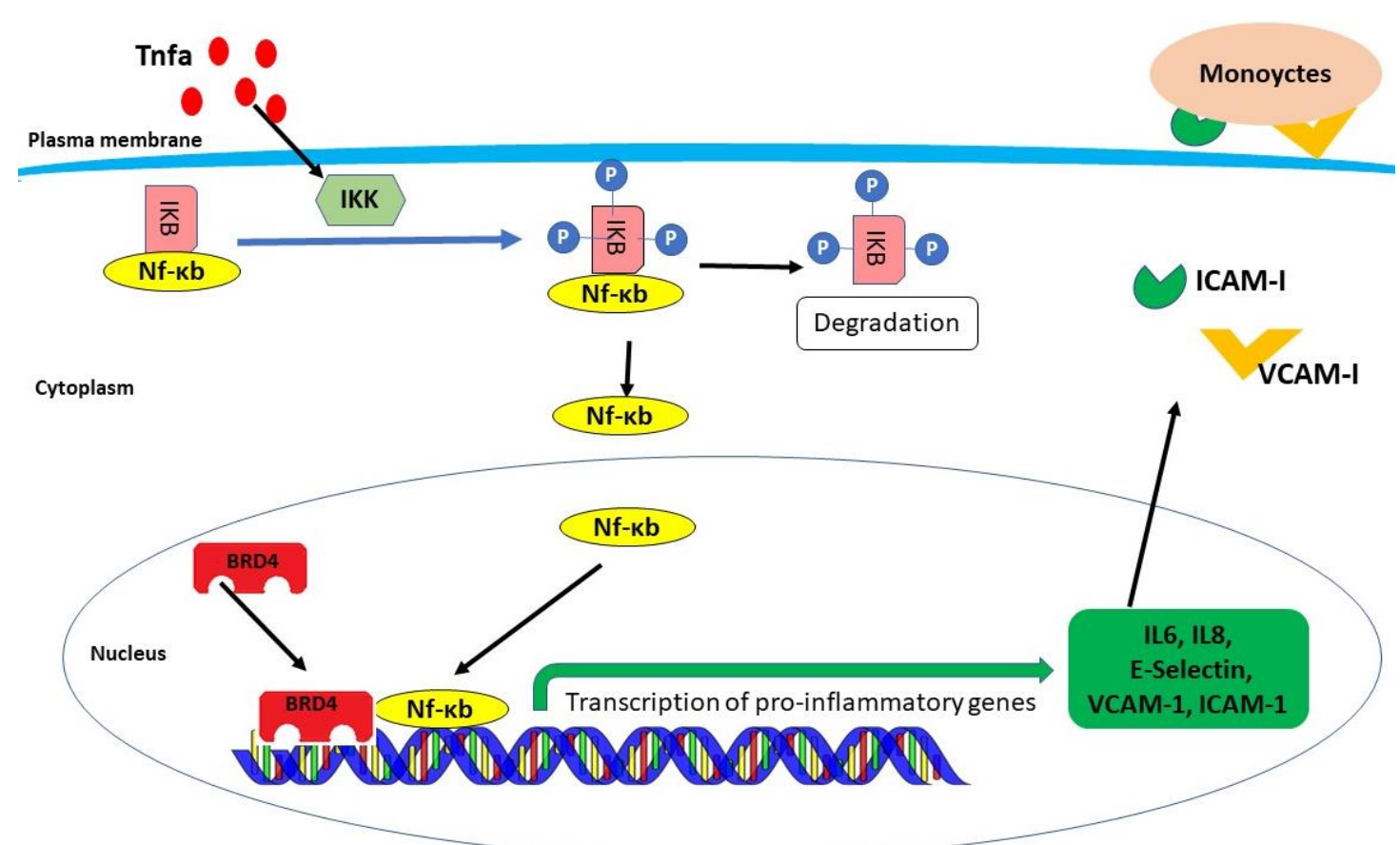

Figure 1.3. NF-kB Signalling pathway. NF-KB heterodimer (RelA and p50) is present in cytoplasm bound with IKB- $\alpha$ in an inactive state. When there is an inflammatory signal, IKK is activated and phosphorylates the IKBa, which is then degraded by the proteasome assembly. The activated NF-KB dimer migrates into the nucleus and binds to chromatin and initiates the transcription of inflammatory markers (E-Selectin, VCAM-I, ICAM-I, IL6, IL8). Adhesion molecules (VCAM-I, ICAM-I) are expressed on activated ECs and binds to monocytes.

Activation of NF-kB pathway is reported in multiple inflammatory diseases like Rheumatoid arthritis, atherosclerosis, multiple sclerosis, asthma and inflammatory bowel disease (Tak and Firestein, 2001). NF-kB is a heterodimer consisting of p50 and p65 (RelA) subunits. In resting state ECs, NF-kB binds to its inhibitor (IKB- $\alpha$ ), which keeps NF-kB in an inactive form (Tanaka and lino, 2016; Xiao et al., 2014). Central step of NF-kB cascade is liberation of NF-kB from IkB- $\alpha$. Inflammatory mediators like TNF- $\alpha$, lipopolysaccharides (LPS) and Interleukin-1 (IL-1) binds to toll like receptors (TL4, TL2) on plasma membrane and activates IKB-Kinase (IKK) (Monaco and Paleolog, 2004). IKK phosphorylates IKB-a, which is subsequently ubiquitinated and degraded by proteasome assembly, and liberates NFKB for nuclear translocation (Monaco and Paleolog, 2004; Xiao et al., 2014). Inside the nucleus, NF-KB interacts with different chromatin modifiers (Histone deacetylases HDACs, p300, BRD4), transcription factors (c-Myc, p53) and binds to enhancer regions of approximately 400 genes to induce their expression (Fig. 1.3) (Tanaka and lino, 2016). 
Multiple pro-inflammatory genes such as cytokines Interleukin 6 and 8 (IL6, IL8), TNF- $\alpha$ and adhesion molecules like SELE, Intercellular Adhesion Molecule 1 (ICAM-1), VCAM-1, which contribute for progression of inflammation and ECs dysfunction are all activated via NF-kB signalling pathway (Tanaka and lino, 2016; Xiao et al., 2014). Among the regulatory partners of NF-KB, Bromodomain containing protein 4 (BRD4), a chromatin modifier has been recently highlighted more due to its important role during inflammation and in atherosclerosis (Picaud et al., 2013; Xu and Vakoc, 2014b).

\subsubsection{Role of BRD4 in atherosclerosis}

NF-KB interacting partners include different transcription factors and chromatin modifiers including BRD4 (Tanaka and lino, 2016). BRD4, an epigenetic regulator, is a member of BET (Bromodomain and extra terminal domain) tandem bromodomain containing family. BET family members contain two N-terminal bromodomains, Bromodomain 1 (BD1) and Bromodomain 2 (BD2) and an Extra terminal (ET) domain (Najafova et al., 2017). BRD4 is known for its role in multiple cellular activities like inflammation, DNA damage repair, spermatogenesis, and cell cycle (Devaiah et al., 2016; Devaiah et al., 2012; Najafova et al., 2017). It is also involved in transcription of oncogenes (Crowe et al., 2016; Najafova et al., 2017). BRD4 has both histone acetyl transferase (HAT) and kinase activity (Devaiah et al., 2016; Devaiah et al., 2012). BRD4 recognizes and binds to acetylated lysine residues of histone proteins, H3 (H3K14 - histone 3 lysine 14) and H4 (H4K5 - histone 4 lysine 5 or H4K12 - histone 4 lysine 12) via BD1 and BD2 (Devaiah et al., 2016).

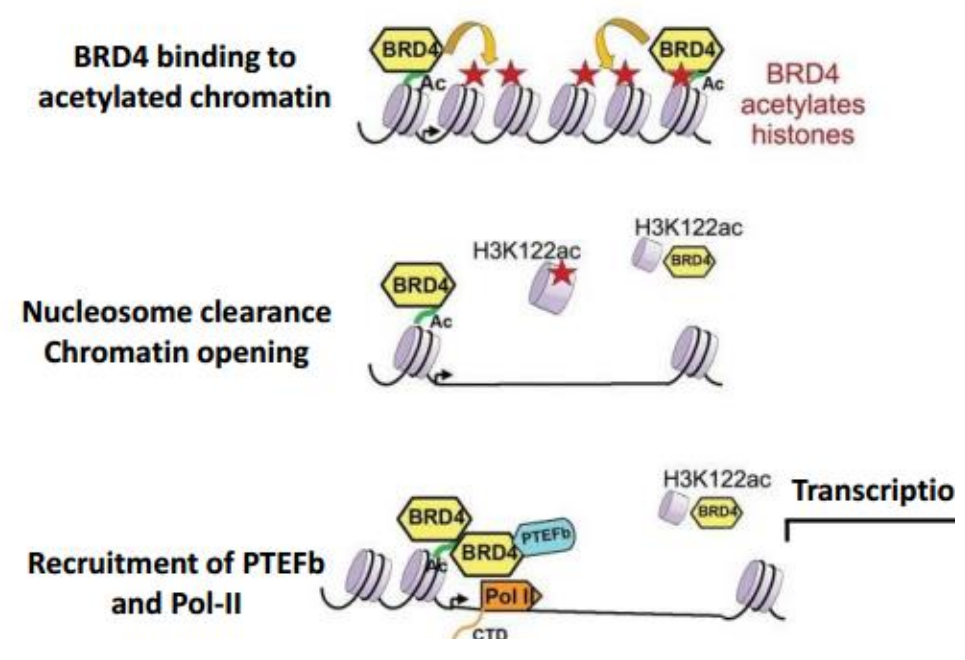

Figure 1.4. Binding of BRD4 on chromatin and acting as HAT and Kinase. BRD4 protein binds to the acetylated chromatin and acetylates histones, leading to DNA-Histone disassembly and opening of chromatin. BRD4 recruits positive transcription elongation factor (P-TEFb) and RNA polymerase 2. BRD4 also acts as atypical kinase and phosphorylates CTD of RNA-Pol II to initiate transcription of target genes (Figure is published by Devaiah et al., 2016). 
After binding to chromatin, BRD4 acetylates the core of histones at $\mathrm{H} 3 \mathrm{~K} 122$ residue and leads to nucleosome (histone-DNA complex) disassembly (Fig. 1.4), followed by recruitment of RNA Polymerase II (RNA Pol-II) (Devaiah et al., 2016). Then C-terminal end of BRD4 protein recruits positive transcription elongation factor (P-TEFB) (Crowe et al., 2016; Devaiah et al., 2012). It has been reported that BRD4 phosphorylates the Serine residue at position 2 (Ser2) of Carboxy-terminal domain (CTD) of RNA Pol-II ensuring proper transcriptional initiation and elongation (Fig. 1.4) (Crowe et al., 2016; Devaiah et al., 2012).

Among the BET protein family members, BRD4 has higher expression in ECs than other BET proteins (Huang et al., 2016). There are two naturally existing splice variants/isoforms of BRD4 known as BRD4 Long (BRD4-L) and BRD4 short (BRD4-S) (Alsarraj et al., 2013; Alsarraj et al., 2011; Crawford et al., 2008). Both BRD4-L and BRD4-S proteins share the same $\mathrm{N}$-terminal region except for last three amino acids, while the $\mathrm{C}$ terminal domain is absent from BRD4-S isoform (Fig. 1.5) (Chiang, 2009). BRD4 isoforms have been reported to act antagonistically in breast cancer progression, where the BRD4-L isoform reduces cancer metastasis, while the BRD4-S enhances the cancer metastasis (Alsarraj et al., 2013). Moreover, BRD4-S isoform is overexpressed in colon cancer cell lines (Alsarraj et al., 2011).

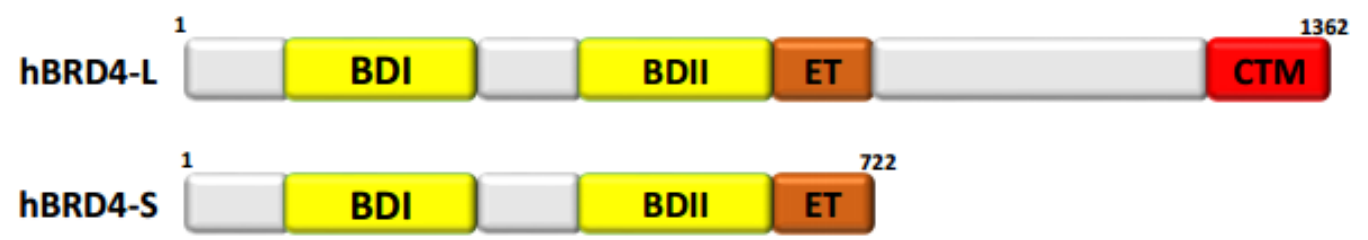

Figure 1.5. Human BRD4 protein isoforms structure. The long isoform of human BRD4 (hBRD4-L) is 1362 amino acid long (152kDa), while the short isoform of human BRD4 (hBRD4-S) is 722 amino acid long (80kDa). Both isoforms share similarity in structure concerning bromodomain I (BDI), bromodomain II (BDII) and an extra terminal domain (ET). However, the long isoform has extra C-terminal region (CTM) which is absent in short isoform.

The evidence that BRD4 has influence on inflammatory response, specifically in atherosclerosis, came up in 2013. In this year, two independent studies revealed that BRD4 was inhibited by a chemical compound, RVX-208, that was already tested for treatment of atherosclerosis by enhancing HDL components APO-A1, but whose exact molecular target till then remained unclear (Picaud et al., 2013; Xu and Vakoc, 2014b). RVX 208 is in clinical 
trials for treatment of diseases like atherosclerosis, Diabetes, Alzheimer's disease, and chronic kidney disease (Drugs.R.D, 2011; McLure et al., 2013; Vidler et al., 2013).

After identifying BRD4 as a target of RVX208, much research was done to identify the exact contribution of BRD4 to atherosclerosis as well as to other inflammatory diseases. In quiescent ECs, BRD4 is known to interact with super enhancers (SEs) of genes that encode for proteins with basal cellular functions (Fig. 1.6). As described above, TNF- $\alpha$ mediated inflammatory stimuli results in the translocation of the NF-kB from cytoplasm to nucleus, which enhances the transcription of pro-inflammatory genes (Brown et al., 2014). Recent studies have uncovered that upon activation of NF-KB, BRD4 redistributes on chromatin from SEs of resting state genes to SEs of pro-inflammatory genes along with NF-KB (Drugs.R.D, 2011; McLure et al., 2013; Xu and Vakoc, 2014b). BRD4 interacts with acetylated Lysine residue (Lysine-310) of the RelA subunit of NF-kB (Huang et al., 2009). NF-KB and BRD4 have been reported to initiate an inflammatory response through up regulation of adhesion molecules and inflammatory cytokines (Fig. 1.6). Chromatin immunoprecipitation (ChIP) with massively parallel DNA sequencing (ChIP-seq) studies on ECs reported that SEs and promoter regions of 271 genes were enriched for RelA and BRD4 after inflammatory stimuli including SELE and VCAM1 (Brown et al., 2014; Xu and Vakoc, 2014b).

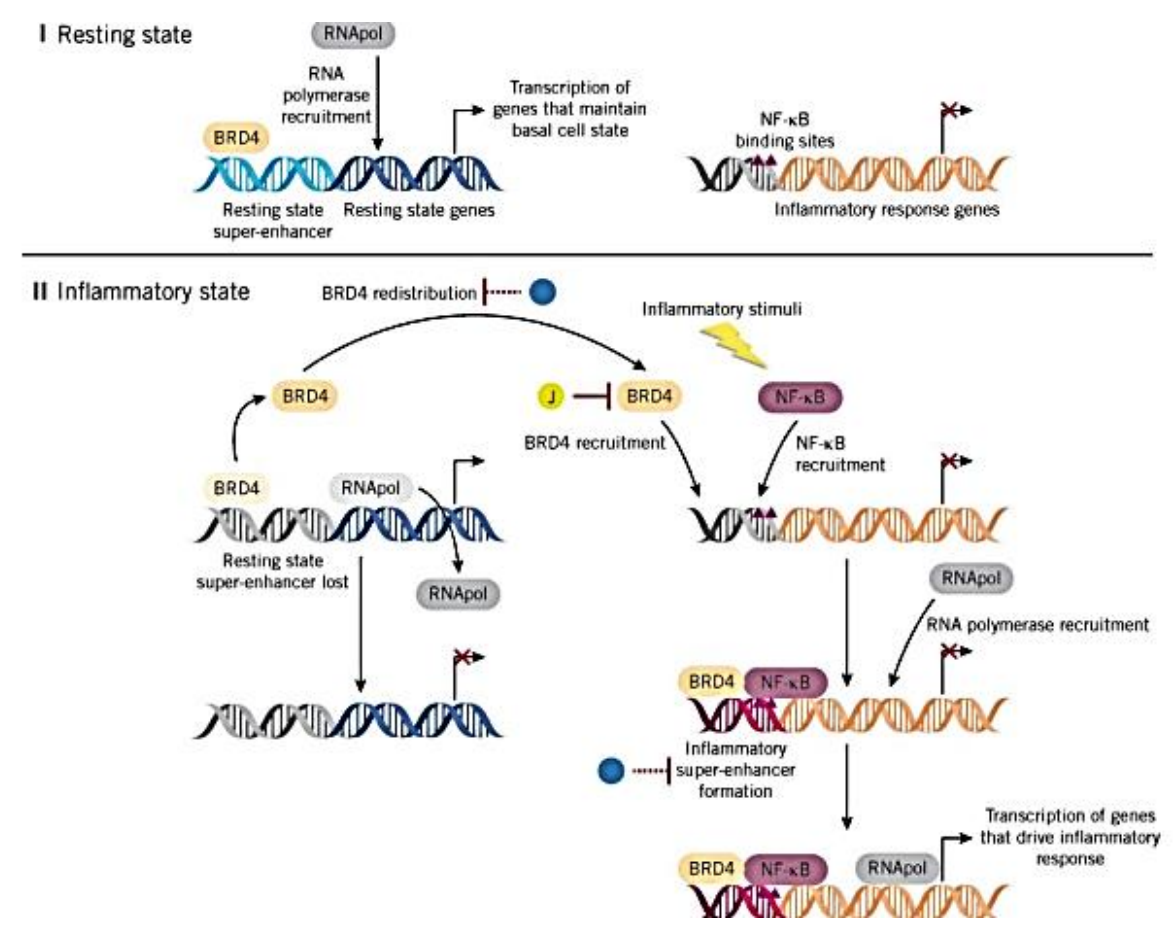

Figure 1.6. Redistribution of BRD4 on chromatin during Inflammatory stimulus. I in normal resting state ECs, BRD4 binds to SEs of resting state genes. II During inflammatory state, BRD4 redistributes on chromatin and bind to SEs of pro-inflammatory genes along with NF-KB. (Figure published by Lou et al., 2014). 


\subsection{Cardiac Hypertrophy}

Cardiac hypertrophy is characterised by thickening and enlargement of heart muscles and shortening of ventricular chambers (Tardiff, 2006). Cardiac hypertrophy occurs due to inflammation and cardiomyocyte growth exceeding the capacity of capillaries to sufficiently supply nutrients and oxygen thus, leading to cardiac hypoxia and cardiomyocytes death. Furthermore, deposition of ECM and fibrosis leads to reduced blood pumping ability of heart (Shimizu and Minamino, 2016; Tardiff, 2006). There are two types of cardiac hypertrophy namely physiological and pathological hypertrophy, which are developed due to extra work load on heart (Tardiff, 2006). Physiological hypertrophy develops in response to pregnancy and excessive exercise, in which cardiac function is enhanced to fulfil the energy demand (Shimizu and Minamino, 2016). Pathological hypertrophy is characterised by myocardial dysfunction, change in sarcomeric structure, hypertrophic and fibrotic ventricle (Fig. 1.7) (Samak et al., 2016).

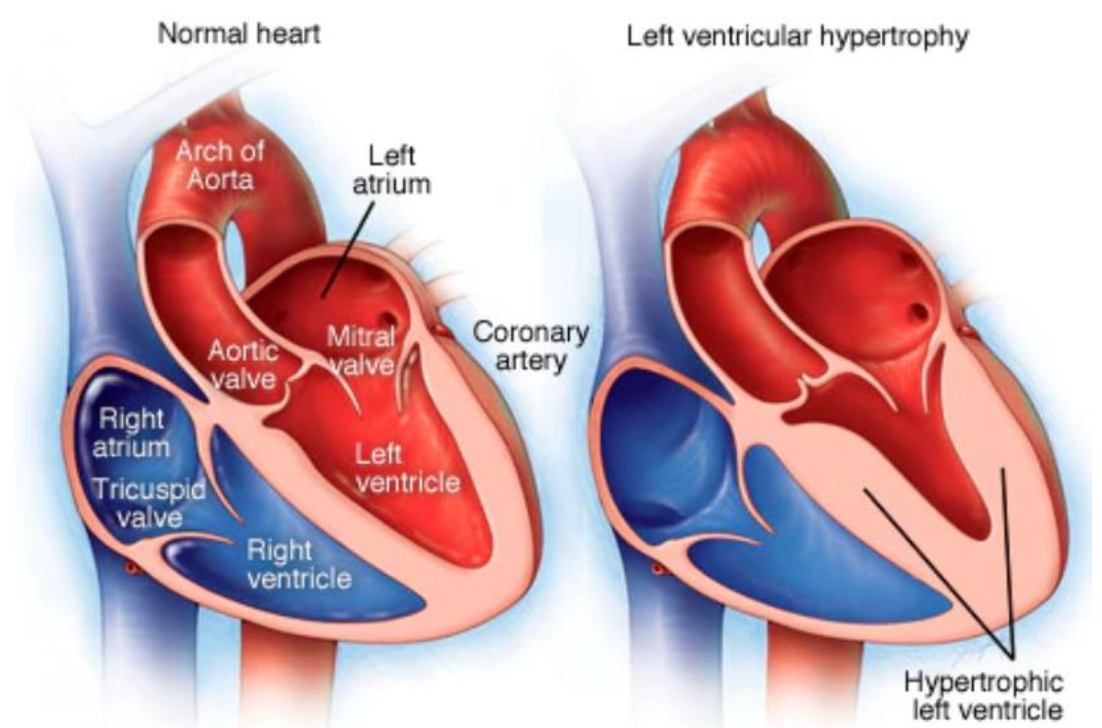

D MAYO FOUNDATION FOR MEDICAL EDUCATION AND RESEARCH. ALL RIGHTS RESERVED.

Figure 1.7. The structure of a normal and cardiac hypertrophy heart. The structure of a normal heart (left) with a normal left ventricular size is shown. The structure of a left ventricular hypertrophic heart (right) showing reduced size of ventricular chamber due to increased fibrosis, increased size of cardiomyocytes and deposition of ECM. (Figure published by Mayo foundation for medical education and research https://www.mayoclinic.org/diseasesconditions/hypertrophic-cardiomyopathy/symptoms-causes/syc-20350198 ).

Multiple pathological conditions such as hypertension, valvular defects, ischaemia, coronary artery disease and diabetes, which increase the energy demand are responsible for the development of cardiac hypertrophy (Heineke and Molkentin, 2006). Initially, cardiac hypertrophy is a compensatory mechanism in response to increased work demand due to 
mechanical and biochemical stress. During this phase, cardiomyocytes become enlarge and increase protein synthesis along with increase in cardiac fibroblasts proliferation (Heineke and Molkentin, 2006). Cardiac fibroblasts, under stress conditions, change their morphology between fibroblast and SMCs and are known as myofibroblasts (Manabe et al., 2002). Myofibroblast cells produce inflammatory chemokines, cytokines and play a significant role in the development of inflammation in hypertrophic heart (Manabe et al., 2002). Prolonged hypertrophy results in cardiac cell death and leads to fibrosis, a condition in which collagen fibres deposits in ventricular chambers, stiffens the ventricles and reduced the contraction and relaxation of heart (Carreno et al., 2006; Manabe et al., 2002). Increased fibrosis leads to reduced oxygen diffusion, which create hypoxic environment resulting in cardiac cell death (Manabe et al., 2002). Stiffness of cardiac tissues and apoptotic conditions leads to impaired cardiac functions (Talman and Ruskoaho, 2016).

\subsubsection{Signalling Pathways in Cardiac Hypertrophy}

Multiple proteins and signalling pathways are known to act as stimulus for cardiac hypertrophy. Activated signalling pathways in physiological and pathological hypertrophy are different. In physiological hypertrophy, fibroblast growth factors (FGF), Ang II, insulin growth factors (IGF) and transforming growth factor $\beta$ (TGF $\beta$ ) activate tyrosine kinase receptors, which further on initiate the phosphoinositol-3-kinase (PI3K) and mammalian target of rapamycin (mTOR) signalling pathways (Fig. 1.8) (Samak et al., 2016). The activation of these signalling pathways leads to increased protein synthesis to compensate work load (Samak et al., 2016).

On the other hand, in pathological hypertrophy activated signalling pathways include MAPKs and JAK, which activate the transcription of maladaptive genes responsible for hypertrophic growth (Carreno et al., 2006; Samak et al., 2016). G-protein-coupled receptors (GPCRs) are also activated in pathological hypertrophy through Ang-II, ET-I (Carreno et al., 2006; Samak et al., 2016). GPCRs activate multiple downstream transcription factors, specifically NF-kB and nuclear factor of activated T cells (NFAT) (Fig. 1.8) (Samak et al., 2016). GPCRs activate voltage-gated calcium ion channels, which leads to elevated levels of calcium ions $\left(\mathrm{Ca}^{+2}\right)$ inside cardiomyocytes (Carreno et al., 2006; Samak et al., 2016). Increased $\mathrm{Ca}^{+2}$ levels are detected by calmodulin (Cam), which leads to dephosphorylation and nuclear translocation of NFAT via activation of calcineurin $A$ and B (CnA, CnB) (Fig. 1.8) (Samak et al., 2016). 


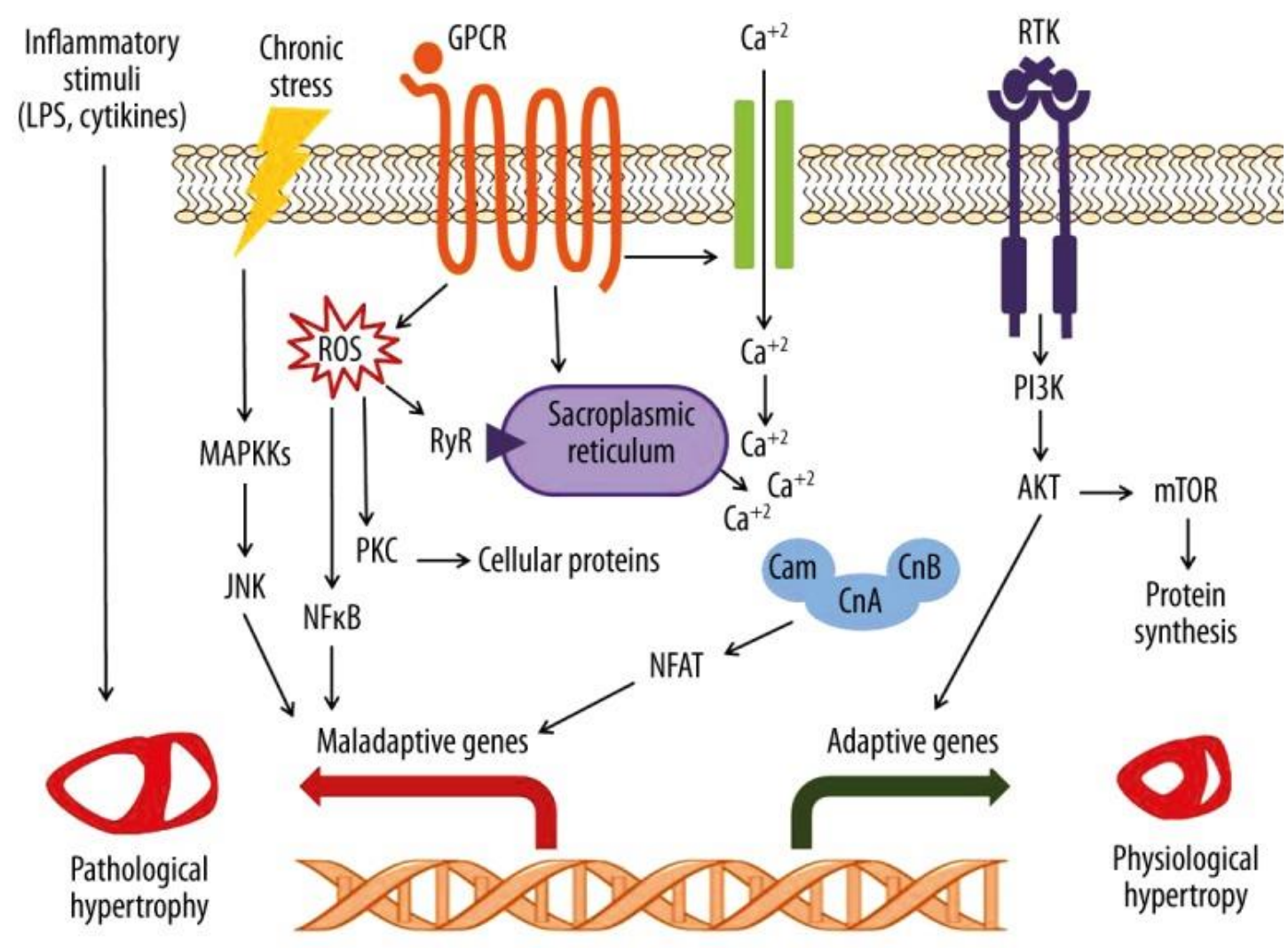

Figure 1.8. Signalling pathways in Cardiac hypertrophy. PI3K and mTOR signalling pathways are activated in physiological hypertrophy while MAPKs, JNK, NFAT and NF-KB are involved in the development of pathological cardiac hypertrophy. (Figure published by Samak et al., 2016)

ROS also contributes to cardiac hypertrophy by inducing apoptosis and activating NF-KB signalling pathway (Fig. 1.8) (Samak et al., 2016). Activation of NF-kB signalling pathway and inflammatory cytokines expression, such as IL6, TNF- $\alpha$ and IL-1 $\beta$, is a hallmark of hypertrophy (Carreno et al., 2006; Samak et al., 2016). The role of NF-kB signalling pathway is not clearly understood, however it is known that it enhances the infiltration of inflammatory cells such as macrophages and T-cells during fibrosis (Samak et al., 2016). Among the targets of NF-KB signalling pathway, Protein Tyrosine Phosphatase 1B (PTP1B) is a protein, reported to have higher expression during inflammation (Zabolotny et al., 2008). 


\subsubsection{PTP1B}

Protein kinases, which are responsible for phosphorylations at serine, tyrosine, threonine, and lysine residues, constitute one of the largest enzyme family encoded by human genome (Manning et al., 2002). Tyrosine phosphorylation is required for activation of receptor tyrosine kinases (RTKs), while protein tyrosine phosphatases (PTPs) are negative regulators of this activation. PTPs were first identified in 1988 when, PTP1B was purified from human placenta (Tonks et al., 1988). The PTP family of enzymes have four classes, classes I, II and III are known as cysteine-based PTPs, whereas class IV is known as aspartate-based PTPs (Alonso et al., 2004; Low et al., 2014). The class I PTPs are further divided into two subgroups namely transmembrane- or receptor-type PTPs and the intracellular PTPs (Barr, 2010). The first intracellular PTP to be purified and characterized was PTP1B (Tonks et al., 1988).

PTP1B has three major domains on the basis of structure and function. The N-terminal catalytic domain is responsible for the phosphatase activity of the enzyme and two flanking Proline rich domains are responsible for protein-protein interactions, while the C-terminal ER targeting domain anchors the enzyme to endoplasmic reticulum (ER) (Fig. 1.9) (Yip et al., 2010).

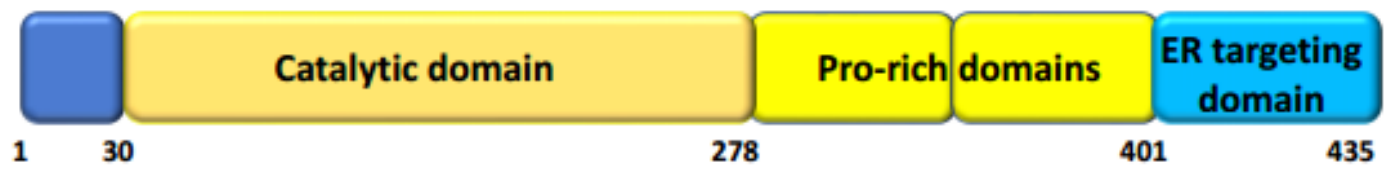

Figure 1.9. Domain architecture of PTP1B. The PTP1b enzyme has three major structural domains namely: Catalytic domain- the main central domain of the enzyme; Proline rich domain- responsible for binding of enzymes to its targets; and ER targeting domain- responsible for attachment of enzyme to ER.

Expression of PTP1B is induced by TNF-a both in vivo and in vitro (Panzhinskiy et al., 2013; Zabolotny et al., 2008). Multiple substrates of PTP1B have been identified and the prominent ones are insulin receptor tyrosine kinase, leptin signalling and vascular endothelial growth factor receptor (VEGFR) (Lanahan et al., 2014; Nakamura et al., 2008). Multiple studies have shown that Ptp1b-knockout (Ptp1b-KO) mice have higher insulin sensitivity, resistant to weight gain even when fed with high fat diet, and have lower level of triglycerides in blood, which is due to higher leptin sensitivity (Elchebly et al., 1999; Tamrakar et al., 2014). Neuronal Ptp1b KO mice are resistant to obesity and have higher 
physical activities, as compared to wild type mice, which become obese and slow, showing that leptin action is controlled by neuronal PTP1B (Bence et al., 2006). Pre-natal and postnatal angiogenesis is a crucial biological process for the development of blood vessels and in wound healing (Ambler et al., 2001; Dormond et al., 2001; Isogai et al., 2001). VEGFR2, a tyrosine kinase receptor, is mainly involved in ECs migration during angiogenesis (Holderfield and Hughes, 2008; Lamalice et al., 2007). Interestingly, PTP1B is a negative regulator of VEGFR2 signalling in ECs (Lanahan et al., 2014; Nakamura et al., 2008). Both in vivo and in vitro studies showed that inhibition of PTP1B function enhances ECs migration and angiogenesis (Besnier et al., 2014; Lanahan et al., 2014).

It has been confirmed that overexpression of PTP1B results in inhibition of VEGFR2 phosphorylation, while endothelial-specific deletion of PTP1B (Endo.Ptp1b.KO) improves VEGF signal transduction (Lanahan et al., 2014; Nakamura et al., 2008). Genome wide gene expression analysis has confirmed the enhanced expression of PTP1B in case of high cardiac afterload (Toischer et al., 2010). Long term application of PTP1B inhibitor or short hairpin RNA (shRNA) mediated inhibition in mice improves angiogenesis, ECs function and reduces cardiac remodelling, making PTP1B as a promising new target to treat CVDs (Gomez et al., 2012).

Previous studies used systemic pharmacological inhibition or genetic deletion of Ptp $1 b$ in mice model, which protects the mice from heart failure after $\mathrm{Ml}$ and improves ECs dysfunction. However, this inhibition or deletion can affect other cell types (cardiomyocytes, fibroblasts) in cardiac remodelling. Therefore, role of ECs remained unclear. To overcome this problem, ECs specific Ptp1b KO (Endo.Ptp1b.KO) mice were generated (Gogiraju et al., 2016). These mice, upon cardiac overload by transverse aortic constriction (TAC), showed reduced oxidative stress, cardiac hypoxia, fibrosis, enhanced angiogenesis, which resulted in improved survival. In this regard, it is interesting to identify novel proteins/pathways, which are involved in PTP1B deficient hearts to device efficient therapies for CVDs. 


\subsection{Aims of the study}

The present study is designed to better understand the role of BRD4 and its isoforms in ECs during inflammation by using in-vitro model of Human umbilical cord derived vascular endothelial cells (HUVECs). The aim is to investigate role of BRD4 isoforms in heart tissues of mice models of aging and cardiac hypertrophy. Moreover, we want to investigate role of BRD4 in monolayer integrity of ECs during inflammation. We also want to analyse PTP1B expression during inflammation and identify the possible mechanism responsible for cardio protection in Endo.Ptp1b.KO hearts using proteomics approach. 


\section{Materials and Methods}

\subsection{Cell Culture}

All in-vitro experiments were performed using primary Human Umbilical cord derived Vascular Endothelial Cells (HUVECs) under sterile conditions. HUVECs were purchased from Promo Cell and cultured using growth medium prepared by mixing Endothelial cells basal medium (Promo Cell) and Endothelial Cell Growth Medium Supplement Pack (Promo Cell), $1 \%$ Penicillin/Streptomycin (Sigma). Cells were cultured on $0.2 \%$ Gelatin coated flasks $/$ plates at $37^{\circ} \mathrm{C}$ in a humidified incubator with $5 \% \mathrm{CO}_{2}$. For trypsinisation, cells were washed with DDPBS (PAN-Biotechnologies) and incubated in Trypsin-EDTA (Gibco) at $37^{\circ} \mathrm{C}$ for 3-5 min. Trypsin activity was inhibited with DMEM medium (PAN-Biotechnologies) containing 10\% FCS (Gibco) and 1\% Penicillin/Streptomycin. Trypsin was removed by centrifugation at $1000 \times \mathrm{g}$ for $5 \mathrm{~min}$. Cells were resuspended in growth medium and transferred to a new flask. Cell counting was performed, when necessary, by using Neubaeur improved chamber. The cells which are at passages between 7 and 9 were used for all the experiments. $5 \times 10^{4} \mathrm{cells} / \mathrm{cm}^{2}$ were used for all treatments unless otherwise stated.

\subsection{Treatments}

\subsubsection{TNF- $\alpha$ treatment}

Human recombinant TNF- $\alpha$ (Peprotech) stock solution $(2.5 \mu \mathrm{g} / \mathrm{ml})$ was prepared in $0.1 \%$ BSA solution. Cells were treated with $20 \mathrm{ng} / \mathrm{ml}$ of TNF- $\alpha$ for $2 \mathrm{hrs}$, then washed with DPBS before fixation or trypsinisation.

\subsubsection{JQ1 treatment}

$5 \mathrm{mg}$ of Bromodomain Inhibitor JQ1 (ApexBio technologies) was dissolved in $109.41 \mu \mathrm{l}$ DMSO (Sigma) to prepare a stock solution of $100 \mu \mathrm{M}$. Cells were treated with $500 \mathrm{nM}$ JQ1 for 4 hrs or 12 hrs depending upon experimental set up. Control cells were treated with equal volume of DMSO, then washed with DPBS before fixation or trypsinisation.

\subsubsection{RVX208 treatment}

$5 \mathrm{mg}$ of Bromodomain inhibitor RVX208 (ApexBio technologies) was dissolved in $1 \mathrm{ml}$ of DMSO (Sigma) to prepare a stock solution of $13.498 \mathrm{mM}$. Cells were treated with 50-, 100- 
, 150- and 200- $\mu \mathrm{M}$ RVX208 for 4 hrs or 30- and 60- $\mu \mathrm{M} 12 \mathrm{hrs}$ depending upon experimental set up. Control cells were treated with equal volume of DMSO, then washed with DPBS before fixation or trypsinisation.

\subsubsection{Claramine treatment}

$5 \mathrm{mg}$ of PTP1B inhibitor Claramine trifluoroacetate salt (Sigma) was dissolved in $1 \mathrm{ml}$ of water to prepare a stock solution of $8.49 \mathrm{mM}$. Cells were serum starved for $2 \mathrm{hrs}$ in ECs medium in absence of serum followed by treatment with 10-, 50- and 100- $\mu \mathrm{M}$ of Claramine for 30 mins, then washed with DPBS before fixation or trypsinisation.

\subsection{Immunofluorescence staining of HUVECs}

Cells were grown on small cover slips coated with $0.2 \%$ Gelatin and treated, as indicated. After completion of the experiment, the culture media was removed, and cells were washed with DPBS. Cells were fixed with 4\% PFA for 30 mins. followed by washing with DPBS 2 times for 10 min each ( $2 \times 10 \mathrm{~min}$ ). Afterwards, blocking was done with $50 \mathrm{mM}$ Ammonium Chloride $\left(\mathrm{NH}_{4} \mathrm{Cl}\right)$ for 10 min at room temperature $(\mathrm{RT})$. Cells were washed ( $3 \times 4 \mathrm{~min}$ ) with permeabilization buffer (0.2\% Triton X-100 (Sigma) in DPBS) followed by incubation at RT with anti NF-KB antibody (cell signalling) prepared at 1:100 dilutions in permeabilization buffer. After $1 \mathrm{hr}$ of incubation, cells were again washed $3 \times 4 \mathrm{~min}$ each with permeabilization buffer. Afterwards, cells were incubated with 1:300 diluted secondary antibody, anti-rabbit IgG conjugated with Alexa 488 (Life Technologies), for $1 \mathrm{hr}$ at RT followed by washing again with permeabilization buffer ( $3 \times 4 \mathrm{~min}$ ) to remove excessive antibody. Finally, cells were washed twice with DPBS and mounted with 4 ,6-Diamidino2Phenylindole, Dihydrochloride (DAPI) (Vector Laboratories). Fluorescent cells were visualised using Axiovert 200M confocal Microscopy (Carl Zeiss).

\subsection{RNA expression analysis}

\subsubsection{RNA extraction from Cells/Tissues}

For total RNA extraction, Trizol (Invitrogen) was used according to manufacturer's instructions. Briefly, HUVECs were treated, washed with DPBS twice and then harvested by trypsinization. $500 \mu \mathrm{l}$ of Trizol reagent was added $/ 10 \mathrm{~cm}^{2}$ and cells were homogenized by vortexing, and total RNA was precipitated using chloroform/isopropanol extraction 
method (Rio et al., 2010). RNA was washed with $70 \%$ Ethanol and air dried, then dissolved in Diethylpyrocarbonate (DEPC) (Sigma) treated $\mathrm{dH}_{2} \mathrm{O}$ and stored at $-80^{\circ} \mathrm{C}$. RNA was quantified using NanoDrop 2000C (PeqLab, ThermoFisher Scientific).

RNA extraction form mice heart tissues was done by pulverizing the tissue in liquid nitrogen using conventional mortar and pistol. Afterwards, pulverized tissues were dissolved in Trizol using a tissuelyser bead mill (Qiagen). The remaining RNA extraction protocol was as described above.

\subsection{2 cDNA synthesis}

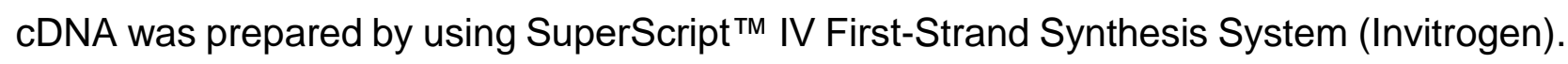
DNase digestion was performed on total RNA before cDNA synthesis. Briefly, $2 \mu \mathrm{g}$ of RNA ideally in $8 \mu \mathrm{l}$ of DEPC water was incubated with $1 \mu \mathrm{l}$ of Amplification grade DNase enzyme (Sigma) and $1 \mu \mathrm{l}$ of 10x DNase reaction buffer (Sigma) for $15 \mathrm{~min}$ at RT. $1 \mu \mathrm{l}$ of Stop solution (Sigma) was added to inhibit the reaction and heated at $70^{\circ} \mathrm{C}$ for $5 \mathrm{~min}$. Then $1 \mathrm{X}$ Reverse Transcriptase buffer (Invitrogen), $0.5 \mu \mathrm{Mol} / \mathrm{L}$ each dNTP (Roche), 13.3U M-MLVRT (Invitrogen) enzyme and $1 \mathrm{U} / \mu \mathrm{l}$ RNase inhibitor (Promega) and $0.15 \mu \mathrm{M}$ Primer $\mathrm{p}(\mathrm{dT}){ }_{15}$ for CDNA synthesis (Roche) were added and incubated at $42^{\circ} \mathrm{C}$ for $1 \mathrm{hr}$. Inactivation of enzymes was done by heating the reaction mix at $70^{\circ} \mathrm{C}$ for $10 \mathrm{~min}$. CDNA was stored at $20^{\circ} \mathrm{C}$.

\subsubsection{Quantitative Real Time Polymerase Chain Reaction (qRT-PCR)}

Primers were designed by using online Primer 3 software. Details of primers used in this study are given in tables 2.1 and 2.2. qRT-PCR reaction mixture was prepared by mixing $1 \mu \mathrm{l}$ of $10 \mathrm{X}$ PCR reaction buffer (Roche), $1 \mu \mathrm{l}$ of DMSO (Sigma), $0.5 \mu \mathrm{l}$ of 1:1000 dilutions of SYBR Green (sigma), $0.2 \mathrm{mmol}$ of each dNTP (Roche), $0.15 \mathrm{U}$ of Taq-Polymerase (produced In-house), $10 \mathrm{pmol} / \mu \mathrm{l}$ of Forward and Reverse primers and $1 \mu \mathrm{l}$ of cDNA (1:10 diluted), volume was made up to $20 \mu$ l with $\mathrm{H}_{2} \mathrm{O}$. PCR conditions to amplify cDNA were set as

- Denaturation, $95^{\circ} \mathrm{C}$ for 2 min one cycle,

- PCR-40 cycles

$>$ denaturation $95^{\circ} \mathrm{C}$ for $30 \mathrm{sec}$,

> Annealing $56^{\circ} \mathrm{C}$ for $30 \mathrm{sec}$,

$>$ Extension $72^{\circ} \mathrm{C}$ for $30 \mathrm{sec}$. 
- Acquisition temperature was different for each primer set (Table 2.1).

PCR reaction were run and analysed using LightCycler 480 (Roche). Data was analysed with LightCycler 480 software SW 1.5.1 (Roche) and values were normalised to housekeeping gene human/mice GAPDH/Gapdh and in case of isoform study BRD4-Total/Brd4total. Comparative Ct method $\left(2^{-\Delta \Delta C t}\right)$ was used to measure relative fold change mRNA expression (Schmittgen and Livak, 2008).

Table 2.1 Human mRNA expression primer list

\begin{tabular}{|c|c|c|c|c|}
\hline Gene name & Direction & Sequence & $\begin{array}{l}\text { Product } \\
\text { Size (bp) }\end{array}$ & $\begin{array}{l}\text { Acquisition } \\
\text { Temp }\left({ }^{\circ} \mathrm{C}\right)\end{array}$ \\
\hline \multirow[b]{2}{*}{ GAPDH } & Forward & TGGGTGTGAACCATGAGAAGTA & \multirow[b]{2}{*}{120} & \multirow{2}{*}{81} \\
\hline & Reverse & GAGTCCTTCCACGATACCAAAG & & \\
\hline \multirow[b]{2}{*}{ SELE } & Forward & СТСTCССTCCTGACATTAGCAC & \multirow[b]{2}{*}{123} & \multirow{2}{*}{81} \\
\hline & Reverse & AGGCTTTTGGTAGCTTCCATCT & & \\
\hline \multirow[b]{2}{*}{ VCAM-I } & Forward & GGAAAAACAGAAAAGAGGTGGA & \multirow[b]{2}{*}{125} & \multirow{2}{*}{79} \\
\hline & Reverse & GCCCATGACACTACATGTCAAC & & \\
\hline \multirow[b]{2}{*}{ IL6 } & Forward & AGTGAGGAACAAGCCAGAGC & \multirow[b]{2}{*}{99} & \multirow{2}{*}{79} \\
\hline & Reverse & GTCAGGGGTGGTTATTGCAT & & \\
\hline \multirow[b]{2}{*}{ BRD4-Common } & Forward & TCCAACCCTAACAAGCCCAA & \multirow[b]{2}{*}{110} & \multirow{2}{*}{81} \\
\hline & Reverse & GAAAGGCCATGCAAACTGGT & & \\
\hline \multirow[b]{2}{*}{ BRD4-Long } & Forward & AGCGAAGACTCCGAAACAGA & \multirow[b]{2}{*}{97} & \multirow{2}{*}{81} \\
\hline & Reverse & TCTGCTGATGGTGGTGATGA & & \\
\hline \multirow[b]{2}{*}{ BRD4-Short } & Forward & TCCTCCAAGATGAAGGGCTT & \multirow[b]{2}{*}{156} & \multirow{2}{*}{81} \\
\hline & Reverse & AGCTTGCTGGGAAGGAATCT & & \\
\hline \multirow[b]{2}{*}{ MIDKINE } & Forward & ACCAGTGCCTTCTGTCTGCT & \multirow[b]{2}{*}{176} & \multirow{2}{*}{81} \\
\hline & Reverse & ATTGTGGGGAAGAACAAAAGC & & \\
\hline \multirow[b]{2}{*}{ p38MAPK } & Forward & CCAGCTTCAGCAGATTATGC & \multirow[b]{2}{*}{246} & \multirow{2}{*}{81} \\
\hline & Reverse & TGGTACTGAGCAAAGTAGGCA & & \\
\hline \multirow[b]{2}{*}{ PTP1B } & Forward & TGGGAAATGCAGGGAGTTCT & \multirow[b]{2}{*}{135} & \multirow{2}{*}{81} \\
\hline & Reverse & TGACTCATGCTTTCGATGCC & & \\
\hline
\end{tabular}


Table 2.2 Mouse mRNA expression primer list

\begin{tabular}{|c|c|c|c|c|}
\hline Gene name & Direction & Sequence & $\begin{array}{l}\text { Product } \\
\text { Size (bp) }\end{array}$ & $\begin{array}{l}\text { Acquisition } \\
\text { Temp }\left({ }^{\circ} \mathrm{C}\right)\end{array}$ \\
\hline \multirow[b]{2}{*}{ Gapdh } & Forward & AGGTCGGTGTGAACGGATTTG & \multirow[b]{2}{*}{129} & \multirow{2}{*}{81} \\
\hline & Reverse & TGTAGACCATGTAGTTGAGGTCA & & \\
\hline \multirow[b]{2}{*}{ Brd4-Common } & Forward & AAGCCTGGAGATGACATCGT & \multirow[b]{2}{*}{148} & \multirow{2}{*}{81} \\
\hline & Reverse & TTGCTGCCCCTGTTTCTTTC & & \\
\hline \multirow[b]{2}{*}{ Brd4-Long } & Forward & AGCCAAGTCCTCAAGTGACA & \multirow[b]{2}{*}{107} & \multirow[b]{2}{*}{81} \\
\hline & Reverse & ТСTССTTСTCTGСАTGCTCA & & \\
\hline \multirow[b]{2}{*}{ Brd4-Short } & Forward & ACGTGATTGCTGGTTCTTCC & \multirow[b]{2}{*}{123} & \multirow{2}{*}{81} \\
\hline & Reverse & CCGTGTCCAATGATTAGGCA & & \\
\hline
\end{tabular}

\subsection{Cell Viability Assay}

Cell viability assay for each experimental set was performed using trypan red cir dye exclusion assay. Briefly, cells were trypsinised and resuspended in $1 \mathrm{ml}$ of HUVEC medium. $5 \mu \mathrm{l}$ of resuspended cells were mixed with $20 \mu \mathrm{l}$ of media and $25 \mu \mathrm{l}$ of $0.5 \%$ trypan blue dye. Then, $10 \mu \mathrm{l}$ of mixture was loaded onto haemocytometer. Viable cells (colourless) and non-viable cells (blue coloured) were counted and percentage of viable cells was calculated.

\subsection{Protein Isolation}

HUVECs were grown and treated as described above. Cells were washed with DPBS and trypsinised. Cell pellets were resuspended by using lysis buffer (Cell Signalling) containing final concentration: $2 \mathrm{mM}$ Tris/ $\mathrm{HCl}, 15 \mathrm{mM} \mathrm{NaCl}, 0.1 \mathrm{mM} \mathrm{Na} 2 E D T A, 0.1 \mathrm{mM}$ Egtazic acid (EGTA), 0.1\% Triton X-100, $0.25 \mathrm{mM}$ sodium pyrophosphate, $0.1 \mathrm{mM} \beta$-glycerophosphate, $0.1 \mathrm{mM}$ Sodium orthovanadate $\left(\mathrm{Na}_{3} \mathrm{VO}_{4}\right), 0.1 \mu \mathrm{g} / \mathrm{ml}$ leupeptin and $1 \mathrm{mM}$ Phenylmethylsulfonyl fluoride (PMSF), EDTA-free protease inhibitor cocktail (Sigma) and Phospho-stop phosphatase inhibitor (Sigma) were added freshly. Lysates were centrifuged at 13000 rpm for $15 \mathrm{~min}$ and clear supernatants (total proteins) were separated. Proteins quantification was done by Bradford method using protein reagent dye (Bio-Rad). 


\subsection{Western blot}

Equal amounts of protein $(35 \mu \mathrm{g})$ were resolved on $4-12 \%$ Bis-Tris SDS-PAGE (sodium dodecyl sulfate-polyacrylamide gel electrophoresis) gels (Thermofisher Scientific) together with molecular weight standards, Proteins were transferred onto nitrocellulose membrane of $0.4 \mu \mathrm{M}$ pore size (Millipore) by using Trans-blot SD cell system (Bio-Rad) for one hour. To avoid non-specific binding, membranes were blocked in blocking buffer: $5 \%$ milk in Trisbuffered saline, containing $50 \mathrm{mM}$ Tris- $\mathrm{HCl}, 200 \mathrm{mM} \mathrm{NaCl}$ and $0.1 \%$ Tween-20 (TBST). Blocked membranes were incubated overnight at $4^{\circ} \mathrm{C}$ with blocking buffer containing indicated primary antibodies against, anti-HSP70-antibody (Cells Signaling \#4872) in 1:1000 dilution, anti-BRD4-antibody (Abcam \# ab84776) in 1:1000 dilution, anti- $\beta$-actinantibody (Sigma \# A5316) in 1:10,000 dilution, anti-MIDKINE-antibody (Abcam \#ab36038) in 1:500 dilution, anti-PTP1B-antibody (Cell Signaling \#5311) in 1: 1000 dilution, anti-AKTantibody (Cell Signaling \#9272) in 1:1000 dilution and anti-p-AKT (Ser 473)-antibody (Cell Signaling \#9271) in 1:500 dilution. Next day, membranes were washed in blocking buffer for 3 times 15 min each followed by incubation with horseradish peroxidase-conjugated (HRP-conjugated) secondary antibody (Bio-Rad) in 1:10,000 dilution for $1 \mathrm{hr}$ at room temperature. Membranes were washed in blocking buffer 3 times, 15 min each followed by washing with DPBS. Protein bands were detected by using chemiluminescence detection method using Chemi-Doc (Bio-Rad). Protein bands were quantified by Image lab software and normalized to either HSP70 or $\beta$-actin.

\subsection{Trans-Endothelial/Epithelial Electrical Resistance (TEER)}

In order to analyse the intactness of HUVECs monolayer, TEER was measured across the monolayer as described previously (Callahan et al., 2004; Kazakoff et al., 1995; Man et al., 2008). Briefly, HUVECs were plated on $0.4 \mu \mathrm{M}$ transwell inserts (Corning CoStar cooperation), coated with collagen, and plated cells at a density $1 \times 10^{5}$ cells/well at passage 4. Achievement of monolayer was confirmed by measuring the TEER across the monolayer. Experiments were performed only when TEER reached the reference value of $>70 \Omega$. $\mathrm{cm}^{2}$ as described previously (Callahan et al., 2004; Kazakoff et al., 1995; Man et al., 2008; Srinivasan et al., 2015). Established monolayers were treated with DMSO (control) for 12 hrs, TNF- $\alpha$ for 12 hrs, RVX208/JQ1 for 12 hrs, pre-treatment with RVX208/JQ1 for 
12 hrs followed by TNF-a for 12 hrs. TEER was measured after 1-, 3-, 6-, 12- and 24-hrs of treatments using the epithelial volt/Ohm meter (EVOM2). Values were normalized against the resistance of blank well coated with collagen and containing only medium.

\subsection{Statistical Analysis}

Statistical analysis of real time, western blot and TEER data was performed using GraphPad Prism 5 software. Three or more independent biological replicates of all experiments were analysed by analysis of variance (ANOVA) and were compared to control groups by using Bonferroni post-test. Statistical significance was analysed by using Student's t-test. All results were plotted in graphs as mean and error bars represent \pm standard error mean (SEM). Probability of less than 0.05 was considered significant (* $\left.p<0.05,{ }^{* *} p<0.01,{ }^{* * *} p<0.001\right)$.

\subsection{Experimental animals}

\subsubsection{Aging Mice}

Wild-type C57BL/6 mice (courtesy of Dr. Muzna Zahur, Viral Vector Lab, Department of Neurobiology, UMG) which were bred under sterile conditions and animals were sacrificed at the age of 1-, 3-, 6-, 8-, 12-, 15- and 18-months. Hearts were immediately frozen in liquid nitrogen.

\subsubsection{Hypertrophic Mice}

The generation and maintenance of inducible Endothelial cell-specific Ptp $1 b$ knock-out (Endo.Ptp1b.KO) and the corresponding control (Endo.Ptp1b.WT) mice were reported previously (Gogiraju et al., 2016). Likewise, the transverse aortic constriction (TAC) or SHAM surgery on 10-12 week old female mice (Endo.Ptp1b.KO and Endo.Ptp1b.WT) was reported previously (Gogiraju et al., 2016). Briefly, mice with loxP flanked Ptp1b allele $\left(P t p 1 b^{f / / f)}\right.$ were bred either with Wild type mice or with transgenic mice expressing a fusion protein of Cre recombinase-estrogen receptor under the control of endothelial receptor tyrosine kinase promoter (Tie2.ERT2.Cre). The off-spring with desired genotype were then fed with Tamoxifen diet to induce Cre recombinase activity. Afterwards, 10-12 weeks-old females with either KO or WT genotype were subjected to TAC or SHAM surgery. In TAC group, a band is placed around the aorta of mice to induce pressure on heart, while SHAM 
(an experimental control) group also underwent surgery, however, aortic arch was exposed but no band was placed around the aorta. This strategy gave rise to four groups of animals namely: 1. Wild-type SHAM (WT-SHAM), 2. Wild-type TAC (WT-TAC), 3. Endothelialspecific Ptp1b knockout SHAM (KO-SHAM), and 4. Endothelial-specific Ptp1b knockout TAC (KO-TAC). After 20 weeks of TAC/SHAM surgery, five independent animals were taken from each group as biological replicates and whole heart tissue lysates were prepared in the presence of protease and phosphatase inhibitors. Afterwards, $50 \mu \mathrm{g}$ of proteins were tryptic digested and the resulting peptides were subjected to liquid chromatography-tandem mass spectrometry (LC-MS/MS) analysis.

\subsection{Global Proteome analysis}

\subsubsection{In-solution digestion}

We performed the in-solution digestion of total proteins essentially as described previously (Leon et al., 2013), with minor modifications. Equal amounts of proteins (50 $\mu \mathrm{g}$ ) from each animal were precipitated with 5 volumes of ice cold $100 \%$ acetone. Pellets were washed with $1 \mathrm{ml}$ of ice cold $80 \%$ ethanol (Waters), dried and resuspended in $0.1 \%$ Rapigest (Waters) solution (Rapigest prepared in $25 \mathrm{mM}$ ammonium bicarbonate solution). Proteins were then reduced using dithiothreitol (DTT) and alkylated with iodoacetamide (IAA). Trypsin concentration of $0.1 \mu \mathrm{g} / \mu \mathrm{l}$ (Promega) was used at 1:40 ratio to Protein: Trypsin (For $1 \mathrm{mg}$ protein $25 \mu \mathrm{g}$ trypsin) to digest the protein. Proteins were digested overnight at $37^{\circ} \mathrm{C}$ with constant shaking. After overnight incubation, formic acid was added to reduce the $\mathrm{pH}$ and to stop the trypsin reaction as well as to degrade Rapigest. Samples were centrifuged at $13000 \mathrm{rpm}$ for $5 \mathrm{~min}$ to pellet down undigested proteins and degraded Rapigest. Supernatants were dried in the speed vacuum concentrator and processed for mass spectrometry analysis.

\subsubsection{LC-MS/MS Analysis}

For mass spectrometric analysis, samples were reconstituted in $2 \%$ acetonitrile/0.1\% formic acid ( $v: v)$ and were analysed as described previously (Khan et al., 2016). Briefly, reconstituted samples were analysed at Q-Exactive hybrid quadrupole mass spectrometer (ThermoFisher Scientific) by using Excallibur 2.4 software. 70,000 full width at half maximum (FWHM) resolution settings were used to acquire MS scan across $350-1600 \mathrm{~m} / \mathrm{z}$. Peptides were selected on basis of following criteria: charge state 2 , or higher, up to 12 
most abundant peptide, intensity threshold of $>2^{*} 10^{-4}$. Followed by isolation at 2.0 FWHM width, fragmentation was done with nitrogen at $25 \%$ collision energy. In order to record the resulted product ion spectra resolution setting was 17,5000 FWHM. Three technical replicates per sample were acquired.

\subsubsection{Bioinformatic analysis of mass spectrometry data}

Peak lists were extracted with Raw2MSMS software in data independent acquisition (DIA) model and the obtained mass spec data was processed using Sequential Window Acquisition of all THeoretical fragment ion spectra (SWATH) analysis. Difference of expression between the groups was observed by Principle Component Analysis (PCA) using MarkerView Software 1.2.1.1 (AB SCIEX). In order to perform cluster analysis, we used Search Tool for the Retrieval of Interacting Genes/Proteins (STRING) database to analyse functional annotation of differentially expressed proteins.

\subsubsection{Welch's t-test based analysis}

After validation of different clusters in PCA, data was further analysed by Welch's t-test to generate lists of differentially expressed proteins (significance level $p<0.05$ ) between all groups. Comparison was done first between WT-SHAM and KO-SHAM groups and then between WT-TAC and KO-TAC groups. Detailed analysis of proteomic data set with Welch's t-test generated lists of upregulated and downregulated proteins in all groups. Volcano plots were generated by using -Log10 of $p$ values (Welch's $t$ test), which were associated with individual proteins, against $\log _{2}$ of fold change in abundance between the WT-SHAM and KO-SHAM, WT-SHAM and WT-TAC, KO-SHAM and KO-TAC, WT-TAC and KO-TAC.

\subsubsection{Ingenuity Pathway analysis (IPA)}

In order to analyse the proteome data with reference to functions of proteins, pathways involved, upstream regulators, downstream effectors, we decided to use Ingenuity Pathway analysis (IPA- Qiagen) in collaboration with Prof. Tuula Anneli Nyman, University of Oslo. The data was uploaded to IPA after calculating $p$-values and fold change using Perseus software. Direct and indirect relationship between the differentially regulated proteins were analysed and supported by previously reported experimentally observed data from human and mice studies. We considered proteins with a $p$ values $<0.05$ and a fold change cut-off value of 1.5 as significant and generated the lists of top canonical 
pathways, differentially abundant proteins and potential upstream regulators based on statistical measurement of differential expression based on the statistical effect size, log2 ratio, and the $\mathrm{P}$ value (Z-Score).

\subsection{Mitochondrial Functional Assay}

In order to analyse the effect of PTP1B inhibition on HUVECs mitochondrial function, we used mitochondrial ToxGlo ${ }^{\mathrm{TM}}$ assay kit (Promega). Briefly, $1 \times 10^{4} \mathrm{cells} / \mathrm{well}$ were plated in 96 well culture plates. Cells were allowed to adhere and grow over night. Next day, cells

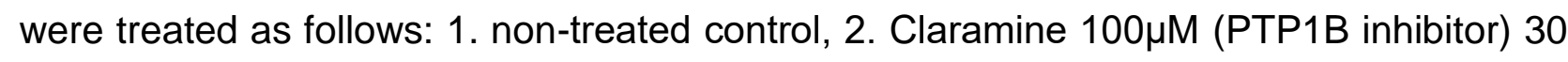
min, 3. TNF- $\alpha$ only (1500/ml for 6hrs), 4. Pre-treatment with Claramine for $30 \mathrm{~min}$ followed by TNF- $\alpha$ treatment for $6 \mathrm{hrs}, 5$. Sodium Azide $100 \mu \mathrm{M}$ for $3 \mathrm{hrs}$.

After completion of treatments, cell membrane integrity was analysed via detection of dead cell protease activity. $5 \mathrm{X}$ cytotoxicity detection reagent was prepared by mixing $10 \mu \mathrm{l}$ of fluorogenic peptide substrate (Promega) with $2 \mathrm{ml}$ of assay buffer (Promega). $20 \mu \mathrm{l}$ of cytotoxic detection reagent was added and incubated at $37^{\circ} \mathrm{C}$ for $30 \mathrm{~min}$. Cytotoxic detection reagent analyse membrane integrity and give fluorescence while interacting with necrosis associated protease. Fluorescence was measured with Victor multilabel counter (Wallac) at $485 \mathrm{nmEx} / 520-530 \mathrm{~nm}$ Em. Average of measured values of non-treated controls were subtracted from average of all treatments and percentage response of each test was calculated.

Next, 2X ATP detection reagent was prepared by mixing $10 \mathrm{ml}$ of ATP detection buffer containing ATPase inhibitors and Luciferase (Promega) with lyophilised ATP detection substrate, luciferin (Promega). $100 \mu \mathrm{l}$ of ATP detection reagent was added to lyse the cells. ATP generates the luminescent signal which was measured with Victor multilabel counter (Wallac). Average of measured values of non- treated controls were subtracted from average of treatments and percentage response of each test was measured. To minimise the ATP production from glycolysis, all treatments were done in DMEM containing Galactose and in the absence of serum to avoid Glucose. 


\section{Results.}

\subsection{Establishment of in-vitro inflammatory model using HUVECs}

In order to establish the in-vitro inflammatory model, HUVECs were treated with or without (control) 20ng/ml TNF-a for 2 hrs. Afterwards, cells were fixed in 4\% PFA, stained with anti-NF-kB antibody and analysed for cellular localisation of NF-kB. As expected, NF-kB was localised in cytoplasm of control cells, however, it translocated inside the nucleus in TNF- $\alpha$ treated cells (Fig. 3.1A). It is known, that upon activation by inflammatory stimuli, NF-kB binds to Super Enhancers (SEs) of pro-inflammatory genes regulatory regions and turn-on the transcription of pro-inflammatory genes like VCAM-I, SELE, IL6 and IL8. Hence, we performed mRNA expression analysis for VCAM-I and SELE, two well-known markers of inflammation, and found significantly induced expression of them in TNF-a treated cells as compared to control cells (Fig. 3.1B). These results confirm the successful establishment of in-vitro inflammatory phenotype in HUVECs.

A

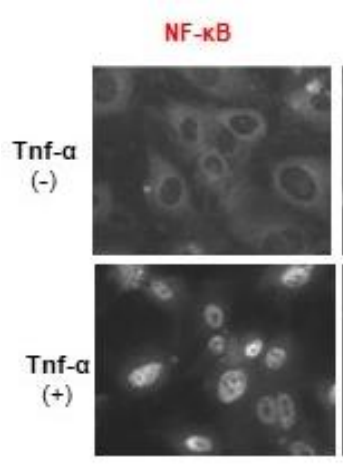

DAPI

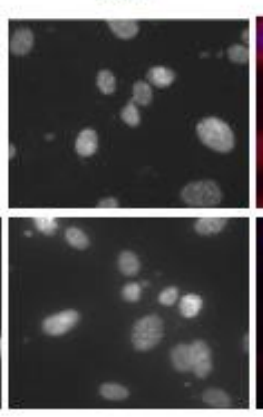

$N F-K B+D A P I$

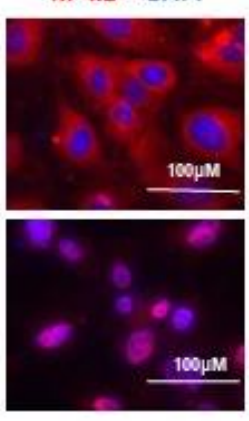

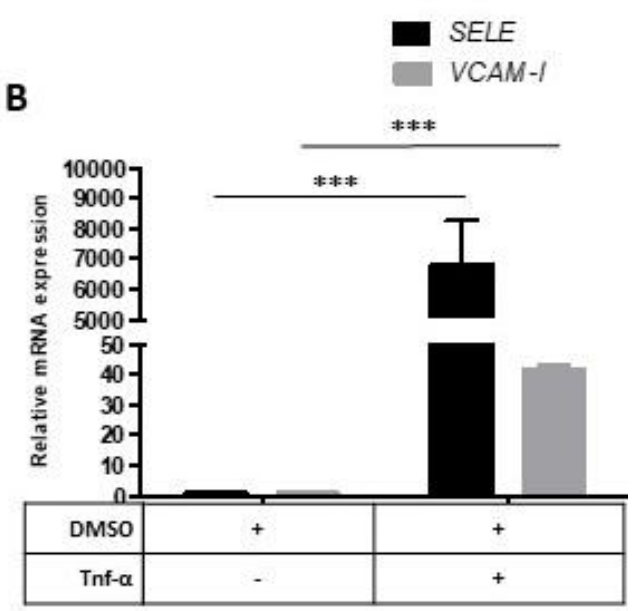

Figure 3.1. Establishment of inflammatory phenotype in HUVECs. A, Immunofluorescence images showing control (-) and TNF- $\alpha$ treated (+) HUVECs which were stained with anti-NF-KB antibody (red) and nuclei were counter-stained with DAPI (blue). B, bar graph showing relative mRNA levels of VCAM-I and SELE after normalization to house-keeping gene GAPDH, in control and TNF- $\alpha$ treated cells. Values are mean \pm SEM of three biological replicates and asterisks indicate statistical significance of ${ }^{* * *} p<0.001$.

After establishment of in-vitro inflammatory model, we decided to analyse the effect of BRD4 inhibition on HUVECs with and without inflammatory stimuli. We wanted to analyse, whether this inhibition is rescuing the HUVECs from inflammatory stress. 


\subsection{Role of BRD4 during inflammation}

It is recently reported that BRD4 interacts with NF-KB and the resulted complex binds to SEs of pro-inflammatory gene regulatory regions during inflammatory response (Brown et al., 2014; Xu and Vakoc, 2014a). Therefore, it is interesting to analyse whether BRD4 inhibition has any significant effect on inflammatory response. Towards this end, we comparatively studied two small molecule inhibitors of BRD4, namely JQ1 and RVX208.

\subsubsection{BRD4 inhibition using JQ1 inhibitor}

The small molecule thienotriazolodiazepine, widely known as JQ1, binds to bromodomains of BET family proteins BRD2, BRD3, BRD4 and BRDT, and inhibits their activity (Bid and Kerk, 2016). Among the BET family members, BRD4 is highly expressed in HUVECs; hence the results obtained with JQ1 might be directly attributable to BRD4 inhibition in these cells (Huang et al., 2016). To establish the concentration of JQ1 required to efficiently inhibit the BRD4 function, we treated HUVECs with different concentrations of JQ1 (50 nM to $1 \mu \mathrm{M}$ ) for $4 \mathrm{hrs}$ followed by 2 hrs of TNF- $\alpha$ treatment. Thereafter, cells were harvested and analysed for the expression levels of pro-inflammatory marker VCAM-I. Our analysis indicated that treatment with $500 \mathrm{nM}$ of JQ1 was more effective in suppressing proinflammatory marker expression in our system (Fig. 3.2A). As these preliminary results are in agreement with the literature (Brown et al., 2014), we decided to use $500 \mathrm{nM}$ of JQ1 in all our further experiments, unless otherwise stated.

Next, we designed an experimental set-up in which HUVECs were treated in four groups:1: Control - cells were treated with DMSO for 4 hrs, 2: TNF- $\alpha$-only - cells were treated with DMSO for 4 hrs followed by TNF-a for 2 hrs, 3: JQ1-only - cells were treated with JQ1 for $4 \mathrm{hrs}$, and 4: JQ1+TNF- $\alpha$ - cells were treated with JQ1 for 4hrs followed by TNF- $\alpha$ for 2 hrs. In order to check any possible cytotoxic effect of TNF- $\alpha$ and JQ1 treatments on HUVECs, we checked the viability of cells after treatments by using Trypan blue dye exclusion assay. These results showed that none of the treatments had any significant cytotoxic effect as compared to control cells (Fig. 3.2B). RNA expression analysis revealed that the control as well as JQ1-only treated cells have basal levels of pro-inflammatory 
markers, whereas TNF- $\alpha$ treated cells showed significantly higher expression of inflammatory markers (Fig. 3.2C).

A

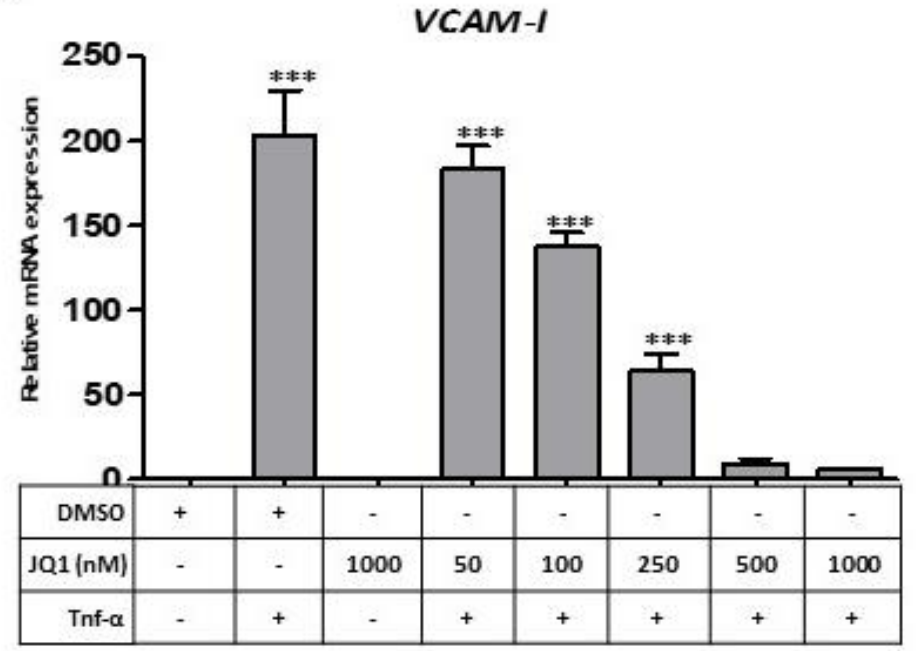

B

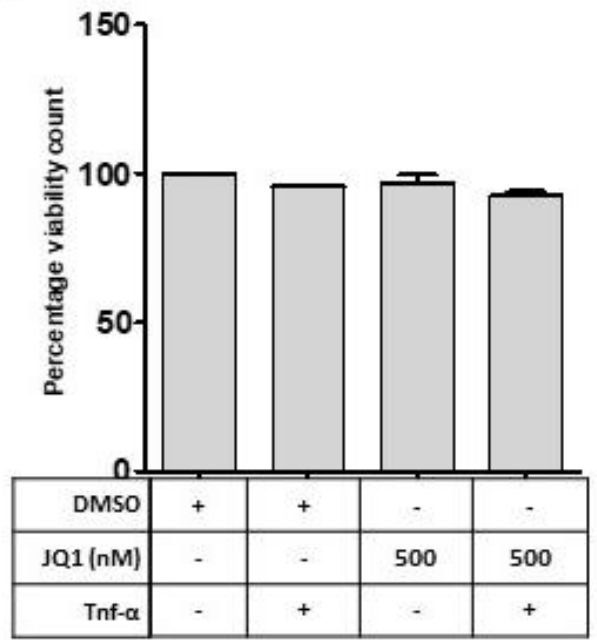

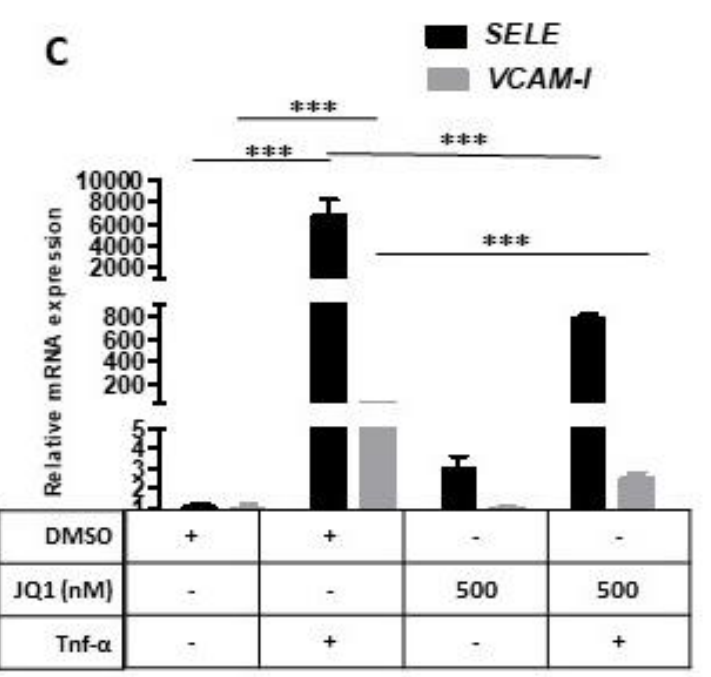
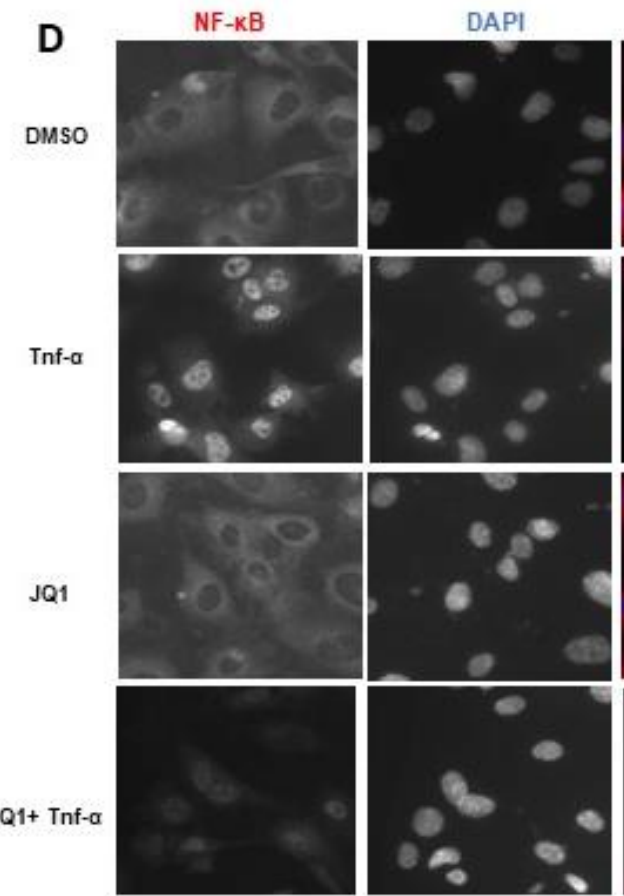

$\mathrm{NF}-\mathrm{KB}+\mathrm{DAPI}$
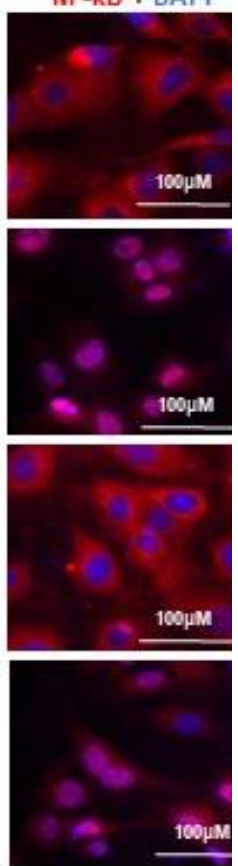

Figure 3.2. BRD4 inhibition using small molecule inhibitor JQ1. A, bar graph showing relative mRNA expression of VCAM-I after normalization to house-keeping gene GAPDH, in HUVECs treated with DMSO (control)-, TNF-a-only, JQ1only- $(1 \mu \mathrm{M})$, and various concentrations of JQ1 (50-, 100-, 250-, $500 \mathrm{nM}$, and $1 \mu \mathrm{M})$ followed by TNF- $\alpha$ treatment. B, bar graph showing viability of cells (\% viability unit) after treated with TNF- $\alpha$-only, JQ1-only and JQ1+TNF- $\alpha$. C, bar graph showing relative mRNA levels of VCAM-I and SELE after normalization to house-keeping gene GAPDH, in DMSO (control), TNF- $\alpha$ treatment, JQ1-only treatment and JQ1 followed by TNF- $\alpha$ treatment. Values in A and C are mean \pm SEM of three biological replicates and asterisks indicate statistical significance of ${ }^{* *} p<0.001$, and ${ }^{* *} p<0.01$. $\mathbf{D}$, Immunofluorescence images of HUVECs treated with DMSO (control)- , TNF- $\alpha$-only- , JQ1-only and JQ1+TNF- $\alpha$ were stained with anti-NF-KB antibody (red) and nuclei were stained with DAPI (blue). 
Interestingly, pre-treatment with JQ1 significantly blocked the expression of proinflammatory markers VCAM-I and SELE by 20 -fold and 9.5 -fold, respectively, as compared to TNF- $\alpha$-only treated cells (Fig. 3.2C). These results indicate successful inhibition of BRD4 function, which results in blocking of its binding to SEs of proinflammatory genes and subsequent inhibition of their expression.

As a next step, we analysed NF-KB localisation in above mentioned treatments. As expected, NF-KB is present in the cytoplasm of control and JQ1-only treated cells, whereas it translocated into the nucleus in TNF- $\alpha$ treated cells. Notably, we found that JQ1 pretreatment did not affect the nuclear translocation of NF-KB (Fig. 3.2D).

Collectively, these results indicate that JQ1 inhibits the function of BRD4 in HUVECs and thereby alleviates the inflammatory phenotype. Although JQ1 has been used in almost all BRD4 inhibition studies, its non-specificity to BRD family proteins and short half-life makes it unsuitable for clinical use (Wadhwa and Nicolaides, 2016). Recent studies identified RVX208 as another BRD4 inhibitor, which covalently binds to BD2-domain of BRD4 (Picaud et al., 2013; Wadhwa and Nicolaides, 2016). Interestingly, RVX208, whose molecular target was not known until recently, was already used in clinical trials for treatment of different diseases like atherosclerosis, diabetes, Alzheimer's disease, and chronic kidney disease (Nikolic et al., 2015; Picaud et al., 2013). Hence, we comparatively studied the efficiency and effectivity of BRD4 inhibition using JQ1 and RVX208 in our inflammatory model.

\subsubsection{BRD4 inhibition using RVX208 inhibitor}

To establish the concentration of RVX208 required to efficiently inhibit the BRD4 function, we treated HUVECs with different concentrations of RVX208 (50-, 100-, 150-, and $200 \mu \mathrm{M})$ for 4 hrs followed by 2 hrs of TNF- $\alpha$ treatment as we established for JQ1 experiments. Thereafter, cells were harvested and analysed for the expression level of pro-inflammatory marker SELE. This analysis showed no expression reduction of SELE in TNF- $\alpha$ treated cells (Fig. 3.3A), indicating that RVX208 probably could not inhibit BRD4 function, even at higher concentration, and after $4 \mathrm{hrs}$ of treatment. Hence, we decided to use time dependent strategy to identify the time and concentration of RVX208 required to inhibit BRD4 function. For this reason, we treated the cells with $30 \mu \mathrm{M}$ and $60 \mu \mathrm{M}$ of RVX208 for 12-, 24-, and 48-hrs followed by treatment with TNF-a. In order to check any possible cytotoxic effect of TNF- $\alpha$ and RVX208 treatment on HUVECs, we checked the viability of 
cells after treatments by using Trypan blue dye exclusion assay. Our results showed that cell viability after 12 hrs of DMSO and RVX208 treatment was better than 24 hrs and 48 hrs (Fig. 3.3B).

A

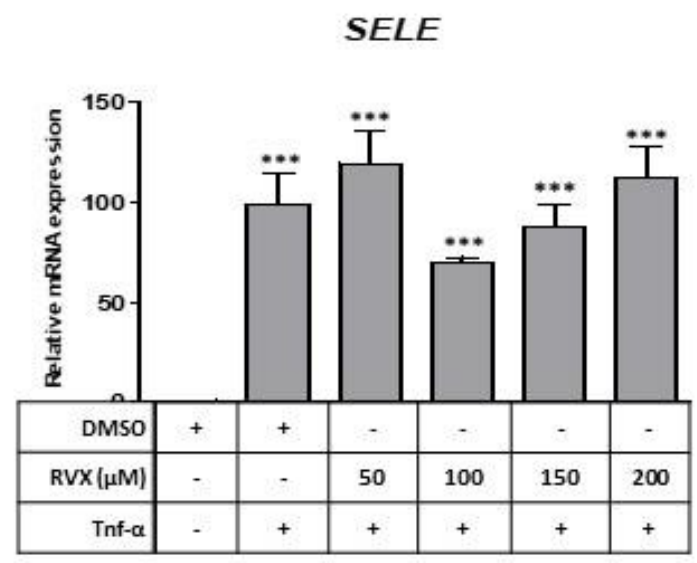

B

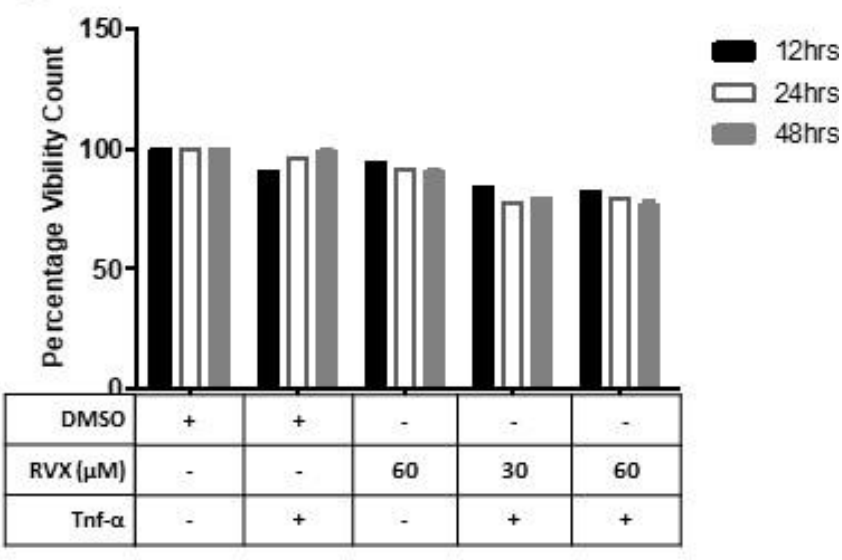

C
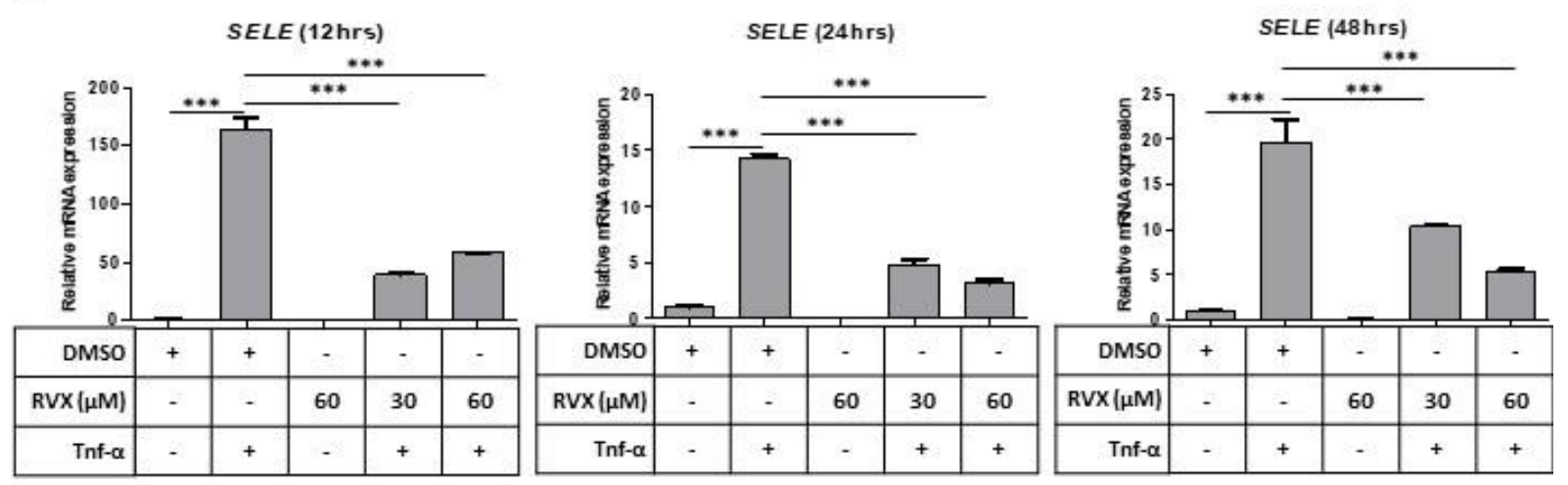

D

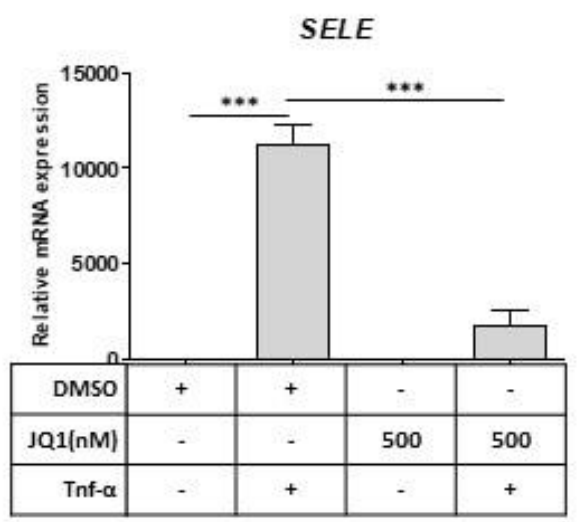

Figure 3.3. Optimization of RVX208 treatment for inhibition of BRD4 function. A, bar graph showing relative mRNA levels of SELE after normalisation to house-keeping gene GAPDH in DMSO (control), TNF- $\alpha$ treatment, RVX208 (50-, 100-, 150-, and $200 \mu \mathrm{M}$ ) followed by TNF-a treatment. B, bar graph shows percentage cell viability count in three different sets 1: 12 hrs set. DMSO (control) 12 hrs, TNF- $\alpha$-only 12 hrs, RVX208-only 12 hrs, RVX208 (30 and $60 \mu \mathrm{M}) 12$ hrs followed by TNF- $\alpha 12$ hrs. 2: 24 hrs set. DMSO (control) 24 hrs, TNF-a-only 12 hrs, RVX208-only 24 hrs, RVX208 (30 and $60 \mu \mathrm{M}$ ) 24 hrs followed by TNF- $\alpha 12$ hrs. 3.48 hrs set. DMSO (control) 48 hrs, TNF- $\alpha$-only 12 hrs, RVX208-only 48 
hrs, RVX208 (30 and $60 \mu \mathrm{M}) 48$ hrs followed by TNF- $\alpha 12$ hrs. C, bar graph showing relative mRNA levels of SELE after normalisation to house-keeping gene GAPDH, in DMSO control, TNF- $\alpha$-only, RVX208-only $30 \mu \mathrm{M}$ and $60 \mu \mathrm{M}(12 \mathrm{hrs}$ to $48 \mathrm{hrs}$ ), and RVX208 (30 $\mu \mathrm{M}$ and $60 \mu \mathrm{M})$ followed by TNF- $\alpha$ treatment. D. bar graph showing relative mRNA levels of SELE after normalisation to house-keeping gene GAPDH, in DMSO control, TNF-a-only, JQ1-only for 12 hrs, JQ1 12 hrs followed by TNF- $\alpha$ treatment for $12 \mathrm{hrs}$. A,C, D, values are mean \pm SEM of three biological replicates and asterisks indicate statistical significance of ${ }^{* *} p<0.001$.

RNA expression analysis indicated that the $12 \mathrm{hrs}$ pre-treatment with RVX208 significantly reduces the expression of pro-inflammatory marker SELE by 4-fold, as compared to TNFa treatment (Fig. 3.3C). Based on these results, we decided to use $60 \mu \mathrm{M}$ of RVX208 for $12 \mathrm{hrs}$ in all our further experiments, unless otherwise stated. As we wanted to perform the comparative studies on RVX208 and JQ1, we first established whether JQ1 could also be used at $500 \mathrm{nM}$ for $12 \mathrm{hrs}$. We treated the cells with $500 \mathrm{nM}$ of JQ1 for $12 \mathrm{hrs}$, TNF- $\alpha$ for $12 \mathrm{hrs}$, and JQ1 for $12 \mathrm{hrs}$ followed by TNF-a $12 \mathrm{hrs}$. Then we performed the RNA expression analysis and found that pre-treatment with JQ1 for $12 \mathrm{hrs}$ could reduce the expression of pro-inflammatory marker SELE by 5-fold (Fig. 3.3D).

After establishing both time and concentrations of RVX208 and JQ1 required to efficiently inhibit the inflammatory response, we designed the following experimental setup in which both RVX208 and JQ1 were compared simultaneously. 1: Control -cells were treated with

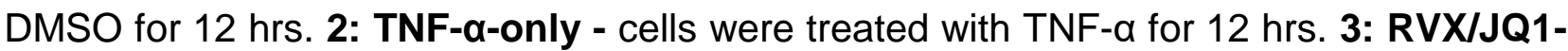
only - cells were treated with either RVX or JQ1 for 12 hrs. 4: RVX/JQ1+TNF- $\alpha$ - cells were pre-treated with either RVX or JQ1 for $12 \mathrm{hrs}$ then with TNF-a for $12 \mathrm{hrs}$. Here we would like to emphasize that, we used the above experimental setup to study the function of BRD4 in almost all treatments to HUVECs, unless otherwise stated. We firstly checked the cell viability of both sets of treatments to confirm that treatments do not have significant cytotoxic effect. This analysis indicates that treatments alone or in combinations were not significantly toxic for cells (Fig. 3.4 A and B). 
A

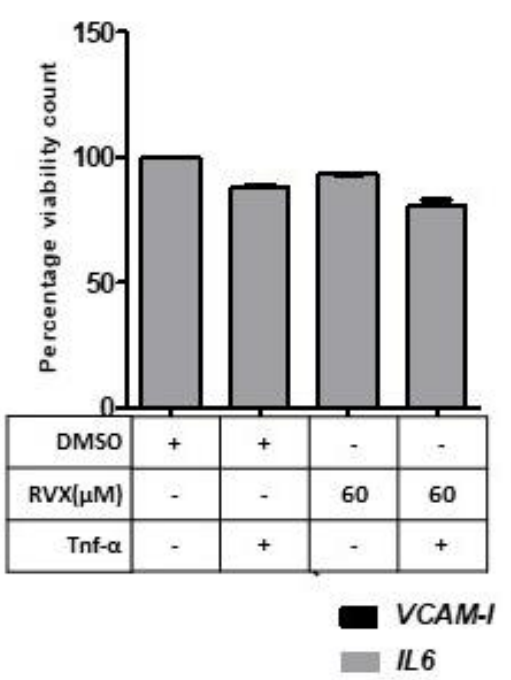

C

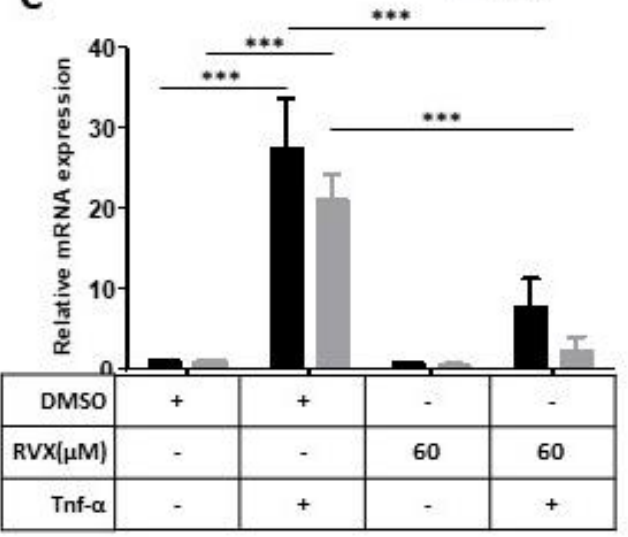

B

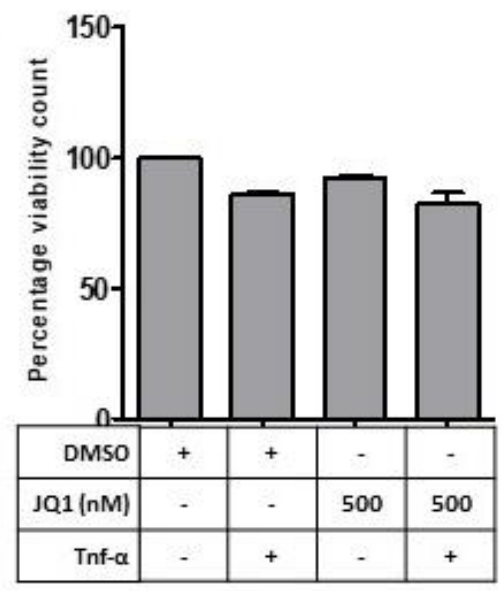

D

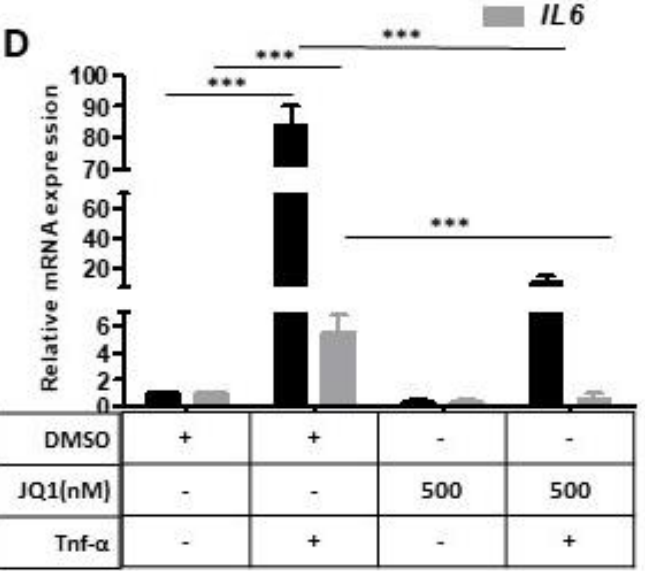

$\mathbf{E}$

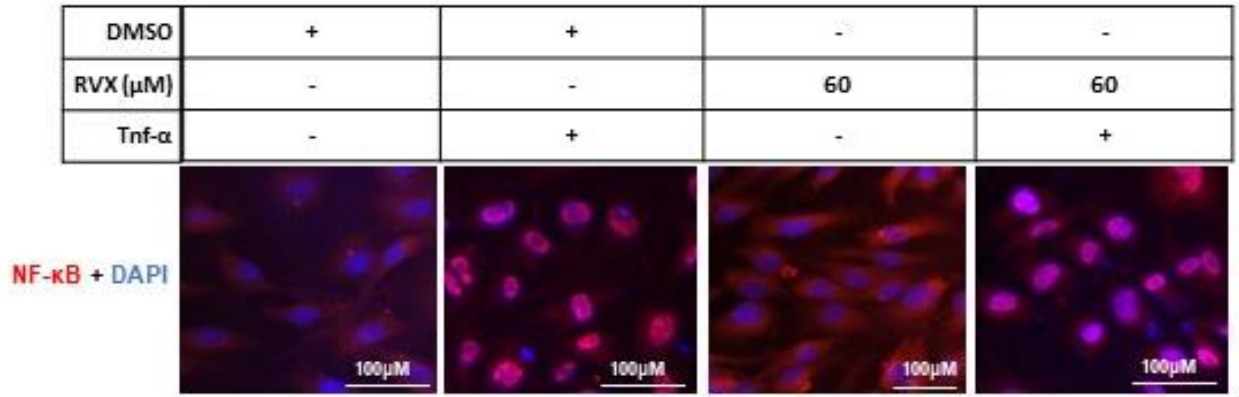

$\mathbf{F}$

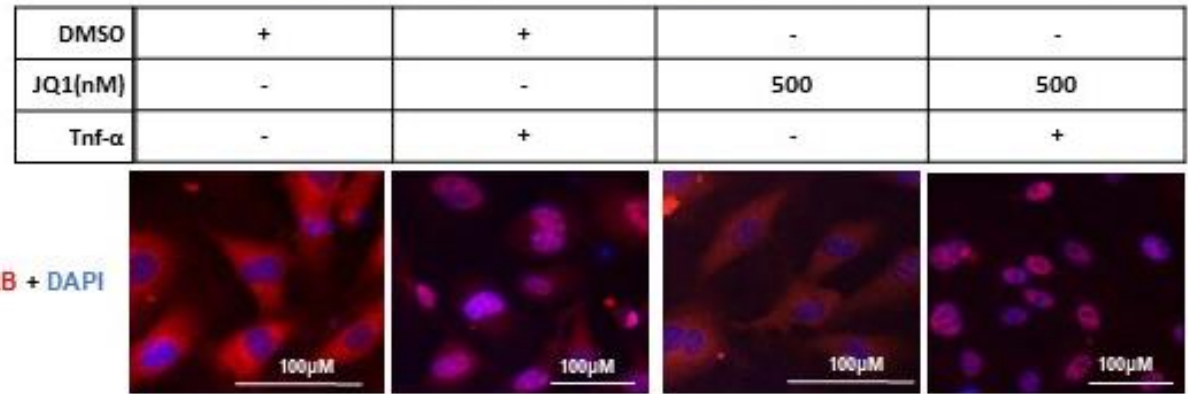

Figure 3.4 BRD4 inhibition using RVX208 and JQ1. A, bar graph shows percentage cell viability count in treatments with RVX208 after 12 hrs treatments. B, bar graph shows percentage cell viability count in treatments with JQ1 after 12 hrs treatments. C, bar graph showing relative mRNA levels of inflammatory markers VCAM-I and IL6 after normalisation to house-keeping gene GAPDH, in HUVECs treated with DMSO, TNF- $\alpha$-only, RVX208-only and RVX208+TNF- $\alpha$ D, bar graph showing relative mRNA levels of inflammatory markers VCAM-I and ILG after normalisation to house-keeping gene GAPDH, in HUVECs treated with DMSO, TNF- $\alpha$-only, JQ1-only and JQ1+TNF- $\alpha$. C-D, values are mean \pm SEM of three 
biological replicates and asterisks indicate statistical significance of ${ }^{* *} p<0.001$. E and $F$, immunofluorescence images for the effect of RVX (E) and JQ1 (F) on the cellular localization of NF-KB in response to indicated treatments. HUVECs were stained with anti-NF-kB antibody (red) and DAPI (blue).

Then we performed the RNA expression analysis and found that pre-treatment with RVX208 could reduce the expression of inflammatory markers by 3 -fold and 7 -fold for VCAM-1 and IL6, respectively, as compared to TNF- $\alpha$ treatment (Fig. 3.4 C). Similarly, pretreatment with JQ1 could reduce the inflammatory markers by 8 -fold and 10 -fold for VCAM$I$ and IL6, respectively, as compared to TNF- $\alpha$ treatment (Fig. 3.4 D). This analysis indicates that both JQ1 and RVX208 could efficiently inhibit the inflammatory response. Moreover, we also noted that the basal expression levels of inflammatory markers VCAM1 and IL6 were reduced by 3-fold and 5-fold, respectively, in JQ1-only treatment as compared to DMSO (Fig. 3.4 C and D). Similarly, RVX208-only treatment is also reducing the basal expression level of inflammatory markers VCAM-1 and IL 6 by 1.4 -fold and 2-fold, respectively, as compared to DMSO.

To confirm that longer incubation (12 hrs) with RVX208 or JQ1 does not have any effect on translocation of NF-KB into the nucleus, we performed the immunostaining for NF-KB localization after above mentioned treatments. This analysis showed that NF-KB is present in the cytoplasm of control and RVX208/JQ1-only treated cells, whereas it translocated into the nucleus in TNF- $\alpha$ treated as well as in RVX208/JQ1+TNF- $\alpha$ treated cells (Fig. 3.4 $E$ and F). Hence, these results proved that RVX208/JQ1 pre-treatment followed by TNF- $\alpha$ does not affect the nuclear translocation of NF-KB.

Collectively, our results show that BRD4 inhibition with either RVX208 or JQ1 suppresses the inflammatory response in HUVECs. In this context, it is interesting to note that BRD4 has two different isoforms namely, BRD4-long (BRD4-L) and BRD4-short (BRD4-S), which have differential roles in different diseases. In particular, the differential expression of BRD4 isoforms is associated with metastasis in colon and breast cancers, in which BRD4$S$ isoform enhances the breast cancer metastasis whereas BRD4-L isoform inhibits metastasis (Alsarraj et al., 2013; Hu et al., 2015). We were interested to determine the expression of BRD4 isoforms in normal as well as in inflammatory states of ECs. Hence, we wanted to study the expression pattern of BRD4 isoforms not only in HUVECs in normal and inflammatory conditions but also during BRD4 inhibition studies and in CVDs models like cardiac hypertrophy and aging hearts. 


\subsection{Elucidating the role of BRD4 isoforms in endothelial dysfunction and in disease models}

\subsubsection{BRD4 isoform analysis in HUVECs}

In order to determine the expression pattern of BRD4 isoforms in tissues of different diseases, we first decided to check the total mRNA levels of BRD4 (BRD4-Total) in our established system. HUVECs were treated as in above described experiments. We found that there is higher expression of BRD4-Total by 1.3-fold to 1.4-fold after 12 hrs of TNF- $\alpha$ treatment as compared control (DMSO). Treatments with either of RVX208 or JQ1 only, reveals a reduced expression of BRD4-Total by 1.6-fold and 1.8-fold in RVX208 and JQ1, respectively (Fig. 3.5A and $\mathrm{B}$ ). These results led us to analyse the isoform specific expression of BRD4 during Inflammatory response or during treatment with RVX28 or JQ1. In order to analyse relative mRNA expression of BRD4- $L$ and BRD4-S isoforms, Isoform specific primers were designed. BRD4- $L$ and $B R D 4-S$ expression was first normalised to housekeeping gene GAPDH and then to BRD4-Total expression. Our data shows that there is no significant difference in expression levels of BRD4- $L$ and BRD4-S isoforms during inflammatory response (TNF- $\alpha$ treatment) (Fig. $3.5 \mathrm{C}$ and D). However, we observed that treatment with RVX208-only or JQ1-only results in reduced expression of BRD4- $L$ by 1.75 -fold and 2.5-fold, respectively (Fig. 3.5 C-D). These results suggest, that the observed reduction in expression levels of BRD4-Total could be due to the reduced expression of BRD4-L. 
A

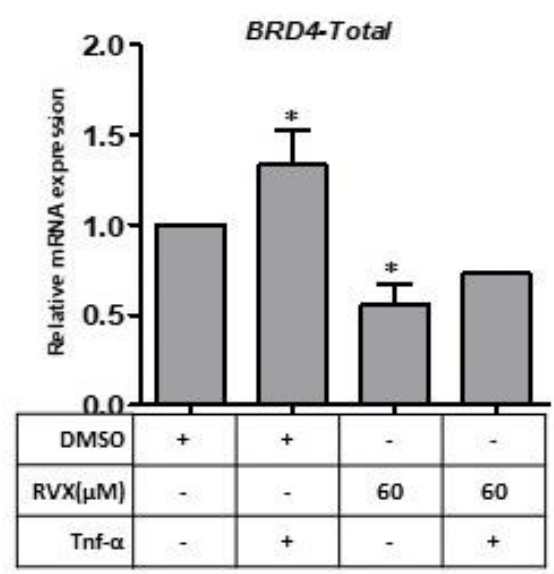

C

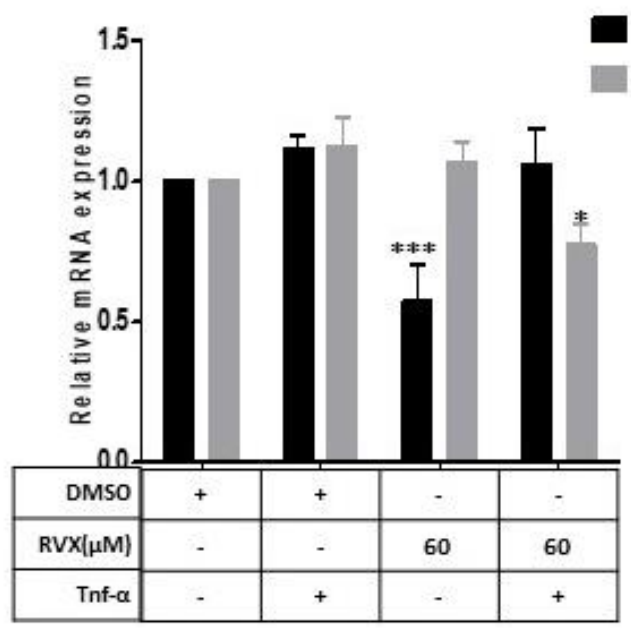

B

BRD4-Total

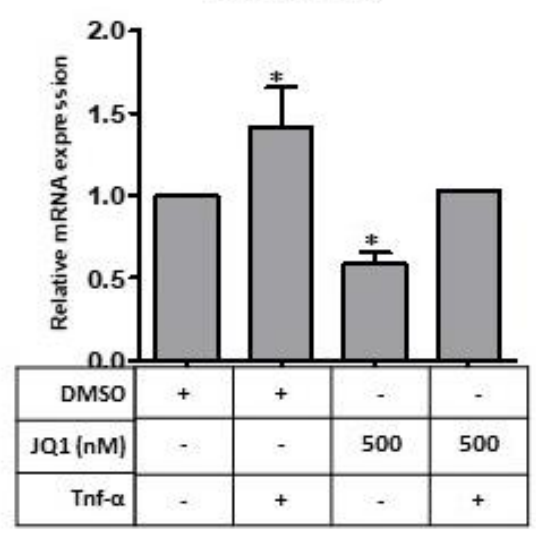

D
BRDAL

BRDAS

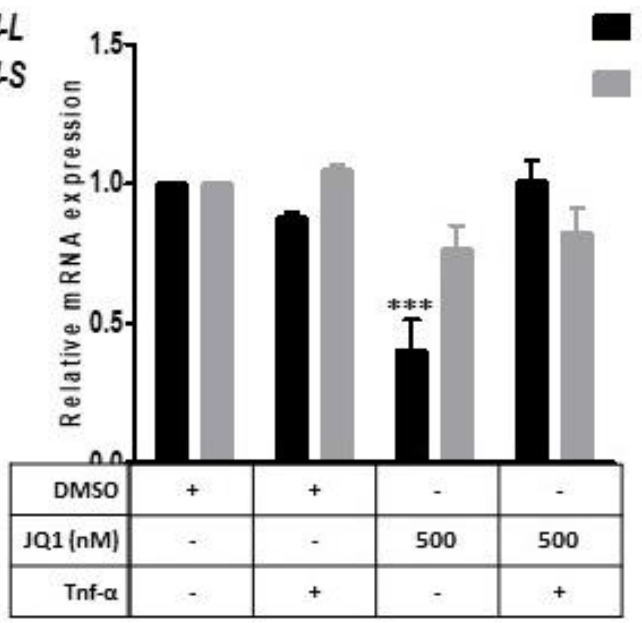

BRD4-L

BRD4-S

Figure 3.5. Expression analysis of BRD4 isoforms in HUVECs. A and B, Bar graph showing relative mRNA levels of $B R D 4-T o t a l$ normalised to house-keeping gene, GAPDH in cells treated with DMSO-only (control), TNF- $\alpha$-only, RVX208only, RVX208+TNF- $\alpha$ (A) or in cells treated with DMSO-only (control), TNF- $\alpha$-only, JQ1-only, JQ1+TNF- $\alpha$ (B). C and D, Bar graph showing relative mRNA levels of $B R D 4-L$ and $B R D 4-S$ isoforms normalised first to house-keeping gene GAPDH and then normalised to BRD4-total in in cells treated with DMSO-only (control), TNF- $\alpha$-only, RVX208-only, RVX208+TNF- $\alpha$ (C) or in cells treated with DMSO-only (control), TNF-a-only, JQ1-only, JQ1+TNF- $\alpha$ (D). Values are mean \pm SEM of two biological replicates and asterisks indicate statistical significance of ${ }^{* *} p<0.001,{ }^{* *} p<0.01$, and * $\mathrm{p}<0.05$.

We have then analysed the expression of BRD4 isoforms at protein level. We treated the cells as mentioned above and performed the Western blot analysis using anti-BRD4 antibody. We found that the cells treated with TNF-a-only have higher expression of BRD4$S$ as compared to control. Similarly, we also found that HUVECs pre-treated with RVX208 
or JQ1 followed by TNF- $\alpha$ treatment show higher expression of BRD4-S isoforms by 1.6fold and 1.4-fold, respectively (Fig. 3.6A-B).

A

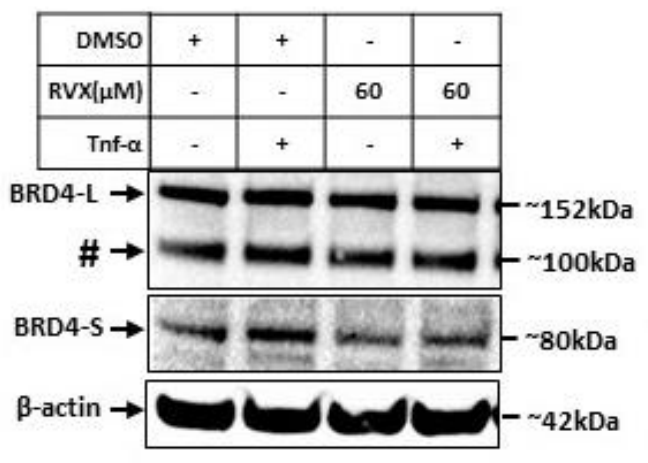

B

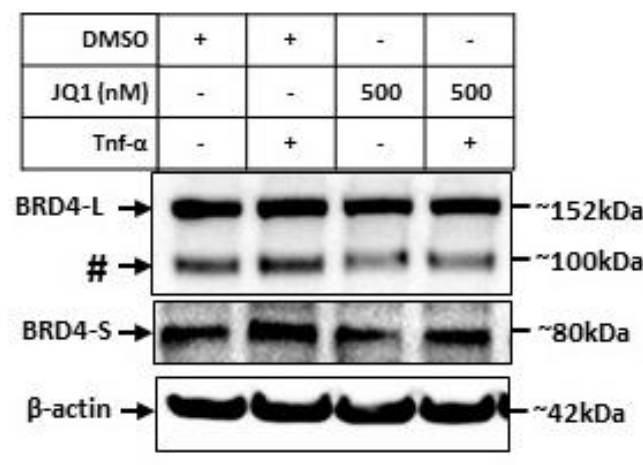

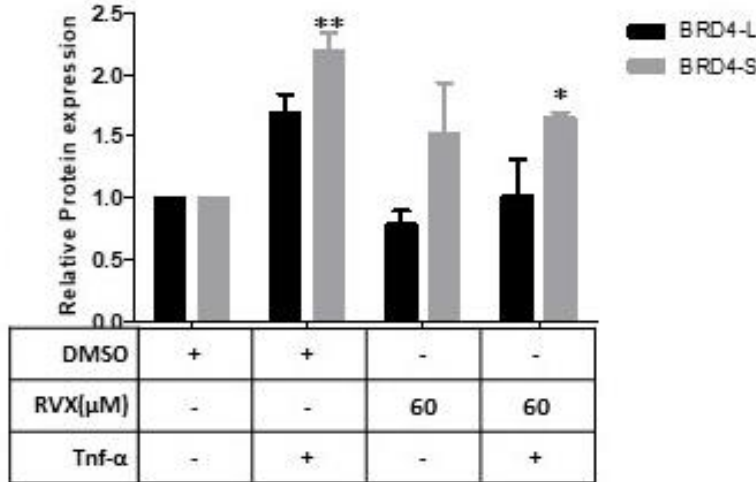

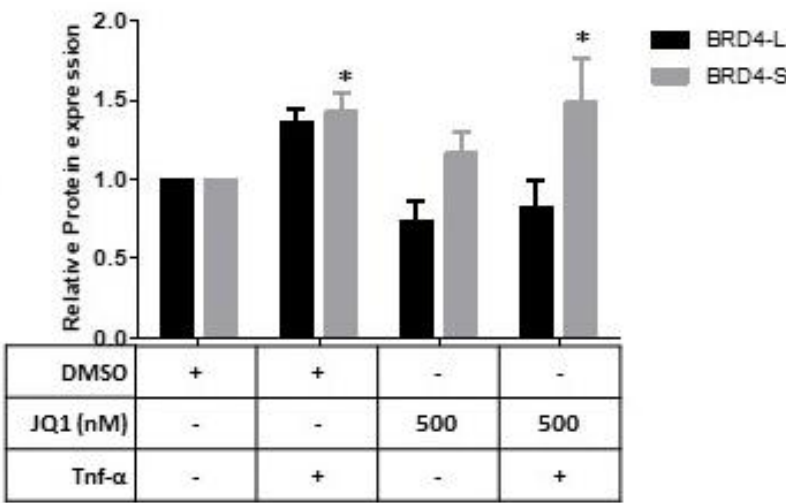

Figure 3.6. Expression analysis of BRD4 protein in HUVECs A-B. Western blot image (left Panels) and the corresponding relative quantification bar graph (right panels) showing the protein expression of BRD4-L and BRD4-S. Immunoblot was stripped and reprobed $\beta$-actin antibody. The $\beta$-actin $(\sim 42 \mathrm{kDa})$ was used as a loading control. The band intensities of BRD4-L and BRD4-S were normalized to $\beta$-actin. The number symbol (\#) indicates a band of 100kDa with an un-known identity detected using BRD4 antibody. Values are mean \pm SEM of two biological replicates and asterisks indicate statistical significance of ${ }^{* * *} p<0.001,{ }^{* *} p<0.01$ and ${ }^{*} p<0.05$.

Collectively, our results indicate that mRNA levels, but not protein levels, of $B R D 4-L$ are reduced when treated with either RVX208- or JQ1-only. In contrast to the RNA data, protein levels of BRD4-S isoform were slightly increased during inflammatory response and it was still high when there is pre-treatment with BRD4 inhibitors, RVX208 or JQ1. Hence, it would be interesting to analyse whether BRD4 isoforms show any specific expression pattern in long-term disease conditions/models as opposed to short-term in vitro studies. Towards this end, we wanted to study the BRD4 isoform expression in CVDs like aging mice hearts and in hypertrophic mice hearts. 


\subsubsection{BRD4 isoforms expression analysis in CVDs models}

It is known that aging increases the risk of CVDs and about $90 \%$ of heart failures are associated with patient older than 70 years (Strait and Lakatta, 2012). Patients older than 65 years have high risk of inflammatory diseases. Expression analyses of inflammatory markers like IL6 and TNF- $\alpha$ are elevated with aging (Kritchevsky et al., 2005). Results showing a high expression levels of BRD4-S isoform during inflammation in HUVECs, led us to analyse expression pattern of BRD4 isoforms in hearts old aging mice. We collected hearts from wild-type C57BL/6 mice at age of 1-, 3-, 6-, 8-, 12-, 15-, and 18-months. Hearts were pulverized in liquid nitrogen and used for RNA isolation. We analysed mRNA expression levels of Brd4-Total (Fig. 3.7A) and Brd4 isoforms (Fig. 3.7B). We found that there is no significant difference of expression of BRD4-Total in hearts of 1- to 18-monthsold mice (Fig. 3.7A). Analysis of isoform-specific expression revealed no significant difference in mRNA expression of both isoforms, except for 6-month-old mice heart, which showed significantly higher Brd4-S expression (Fig. 3.7B). At the protein level, BRD4-L expression was similar in all samples, but significantly higher in hearts of 18-month-old mice (Fig. 3.7C). However, BRD4-S expression is only detectable till 8-months old mice heart (Fig. 3.7C).

It is known that there is an increase in expression of inflammatory genes in pressure overloaded hearts (Chen et al., 2015; Souders et al., 2012). In this context, it would be interesting to analyse whether BRD4 has any expression changes in pressure overloaded TAC and its experimental control, SHAM, mice hearts. To this end, total proteins were isolated from TAC and SHAM mice hearts 20 weeks after surgery and subsequently subjected to western blotting analysis. We found that there was no significant expression difference in BRD4-L and BRD4-S isoforms in TAC and SHAM mice hearts (Fig. 3.7D). 
A

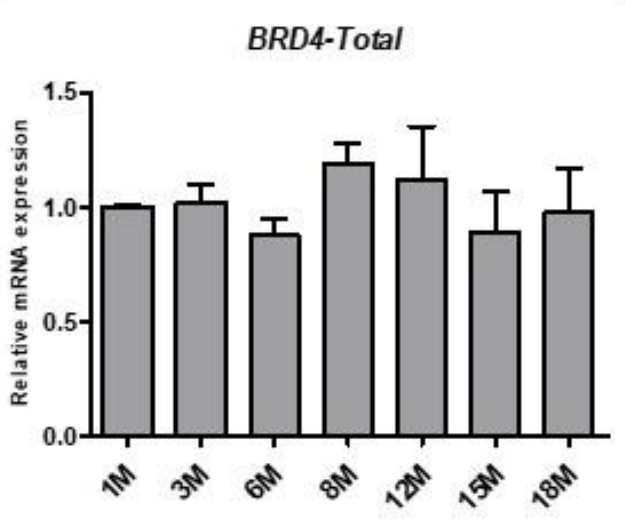

B

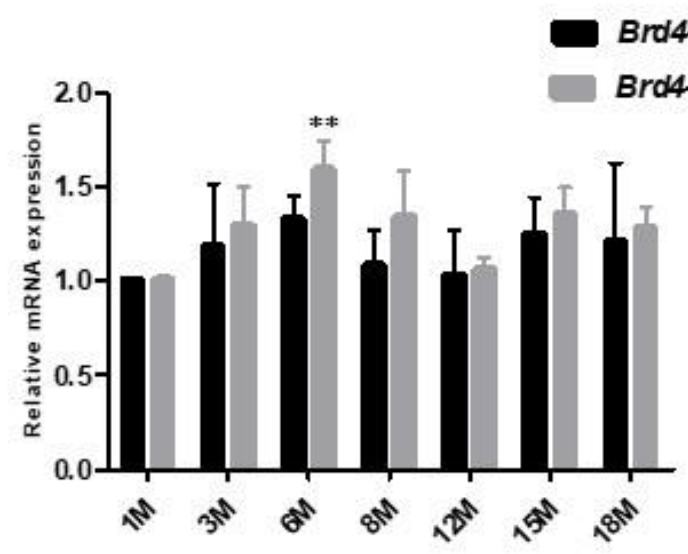

Aging mice hearts
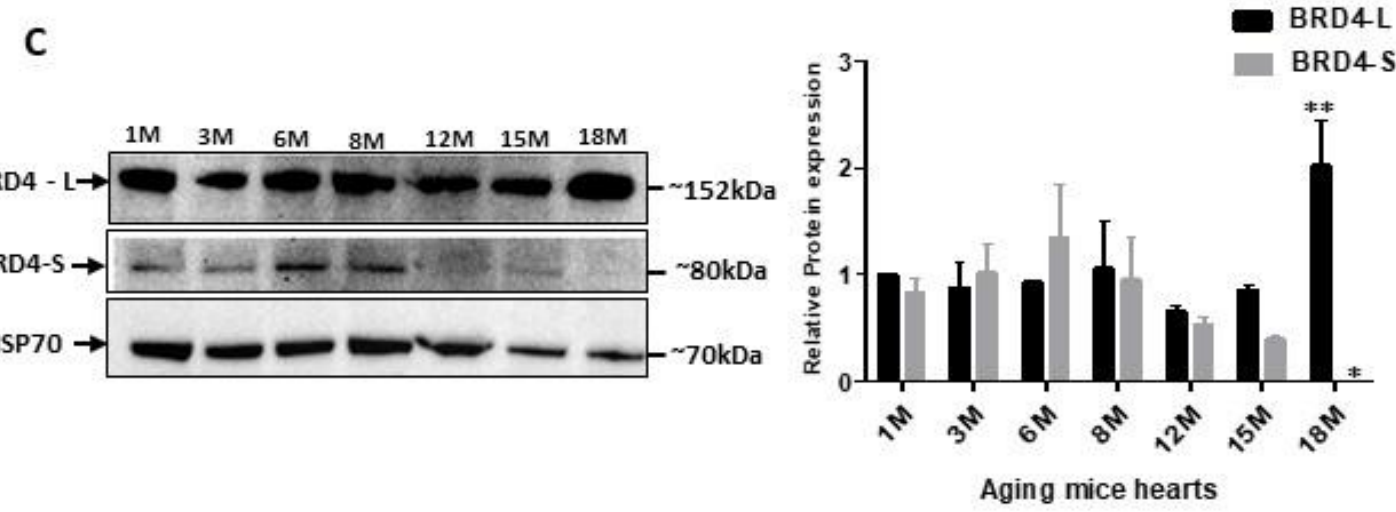

D
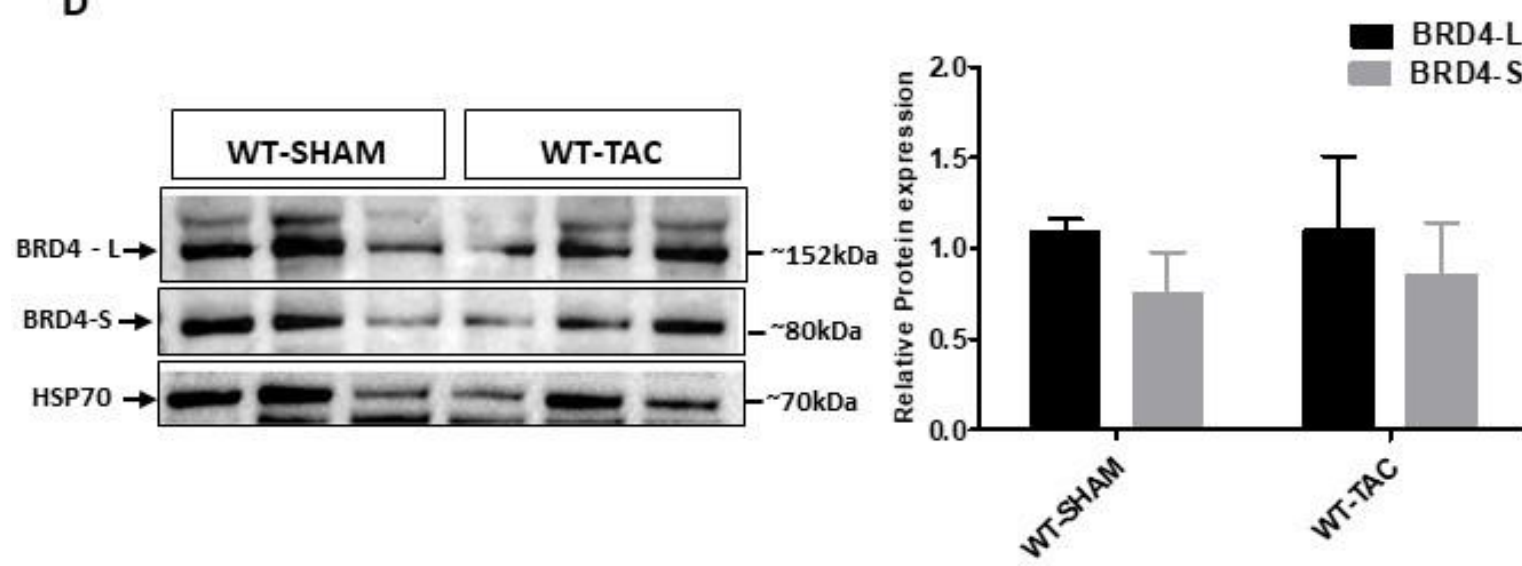

Figure 3.7. Expression of BRD4 isoforms in mice hearts. Bar graph showing relative mRNA level of Brd4-Total normalised to house-keeping gene, Gapdh $(\mathbf{A})$ and, $B r d 4-L$ and $B r d 4-S$ isoforms normalised first to house-keeping gene Gapdh and then normalised to Brd4 total (B) in 1-, 3-, 6-, 8-, 12-, 15-, and 18- months old mice hearts. C and D, Western blot image (left Panels) and the corresponding relative quantification bar graph (right Panels) showing the protein expression of BRD4-L and BRD4-S in 1- to 18-months old mice (C) and in WT-SHAM and WT-TAC mice hearts (D). Immunoblotting for $\beta$-actin was used as a loading control. The band intensities of BRD4-L and BRD4-S were normalized to $\beta$-actin. Values are mean \pm SEM of three (two in $B$ ) biological replicates and asterisks indicate statistical significance of ${ }^{* *} p<0.01$ and ${ }^{*} p<0.05$.

In mouse models of CVDs, we found indecisive results that BRD4-L isoform expression is high in $18 \mathrm{M}$ old mice as compared to earlier stages. However, BRD4-S was not detectable 
after 8-months of age. Likewise, there was no significant difference of BRD4-L and BRD4$S$ isoforms in cardiac hypertrophic mice hearts (TAC) as compared to SHAM mice. This data may indicate that BRD4 isoforms expression do not vary during cardiac remodelling of hearts in hypertrophy. After expression analysis of BRD4 isoforms, we further investigated the role of BRD4 in monolayer integrity of ECs.

\subsection{Identifying the role of BRD4 and Midkine in monolayer integrity of endothelium}

\subsubsection{Effect of inflammatory phenotype on HUVECs monolayer integrity}

It has been reported that TNF- $\alpha$ treatment results in increased permeability and monolayer disruption in vascular ECs (Friedl et al., 2002; Royall et al., 1989). Likewise, retinal ECs also lose monolayer integrity because of disruption of tight junctions via activation of NFKB signalling (Aveleira et al., 2010). In order to analyse the effect of TNF- $\alpha$ treatment on HUVECs monolayer integrity, we used Trans-Endothelial/Epithelial Electrical Resistance (TEER) measurement test and the change in electrical resistance as an indicator of monolayer integrity. To perform TEER measurements, HUVECs were grown on $0.4 \mu \mathrm{M}$ pore size transwell membranes for 3-5 days until cells formed the monolayer and showed a reference value of $\sim 80 \Omega . \mathrm{cm}^{2}$ (Callahan et al., 2004; Kazakoff et al., 1995; Man et al., 2008; Srinivasan et al., 2015). Afterwards, HUVEC monolayers were treated with either with DMSO or TNF-a for 12 hrs and measured for electrical resistance at different time intervals (0-, 3-, 6-, and $12 \mathrm{hrs}$ ). There was a significantly reduced electrical resistance across TNF- $\alpha$ treated monolayer compared to control (DMSO) treated monolayer, indicating increased permeability and loss of monolayer integrity in TNF- $\alpha$ treated cells (Fig. 3.8). 


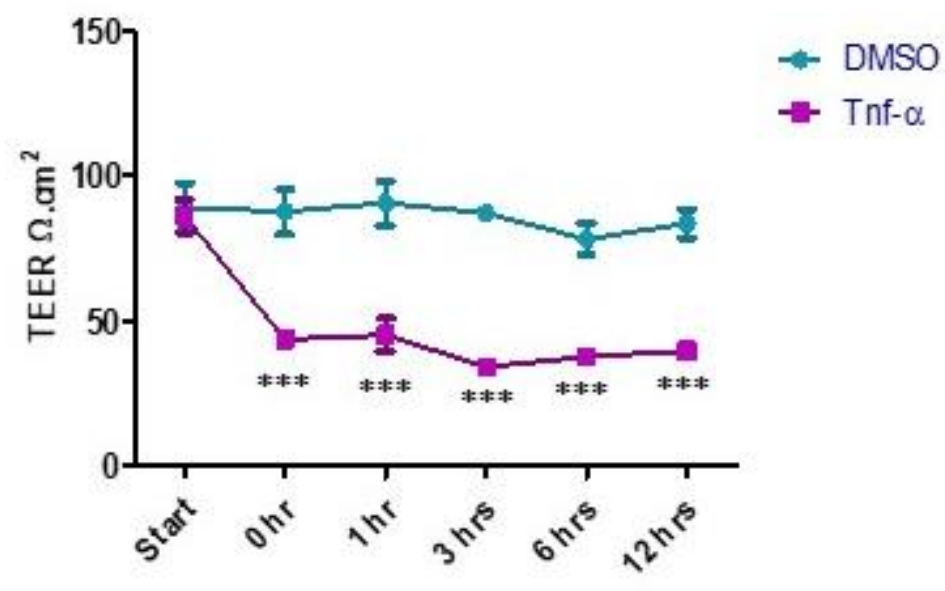

Figure 3.8. Disruption of monolayer integrity in HUVECs : HUVECs were grown on $0.45 \mu \mathrm{M}$ transwell membrane for 5 days to establish the monolayer. Line graph shows Transepithelial/endothelial electrical resistance (TEER) measured in HUVECs monolayer treated with DMSO and TNF- $\alpha$ for $12 \mathrm{hrs}$. Values are mean \pm SEM of three biological replicates and asterisks indicate statistical significance of ${ }^{* *} p<0.001$.

Next, we were interested to study whether BRD4 inhibition could preserve the monolayer integrity in TNF- $\alpha$ treated monolayer as BRD4 inhibition is shown to reduce the inflammatory response, which is known to be associated with monolayer disruption.

\subsubsection{BRD4 inhibition protects HUVECs monolayer integrity}

To analyse the effect of BRD4 inhibition on monolayer integrity, HUVECs monolayers were treated with DMSO-only-, TNF- $\alpha$-only-, RVX208-only-, and RVX208 followed by TNF- $\alpha$. Similarly, the second set of monolayers was treated with JQ1 instead of RVX208. After the indicated treatments, the monolayers were subjected to TEER measurement at different time intervals. As expected, TNF- $\alpha$ treated cells showed significantly reduced electrical resistance as compared to control (DMSO). Surprisingly, when HUVEC monolayers were pre-treated with JQ1 or RVX208 followed by treatment with TNF- $\alpha$, we found no change in 
resistance across the monolayer (Fig. 3.9A-B). Hence, these results indicate that BRD4 inhibition is also protecting the HUVECs monolayer integrity from inflammatory response.

A

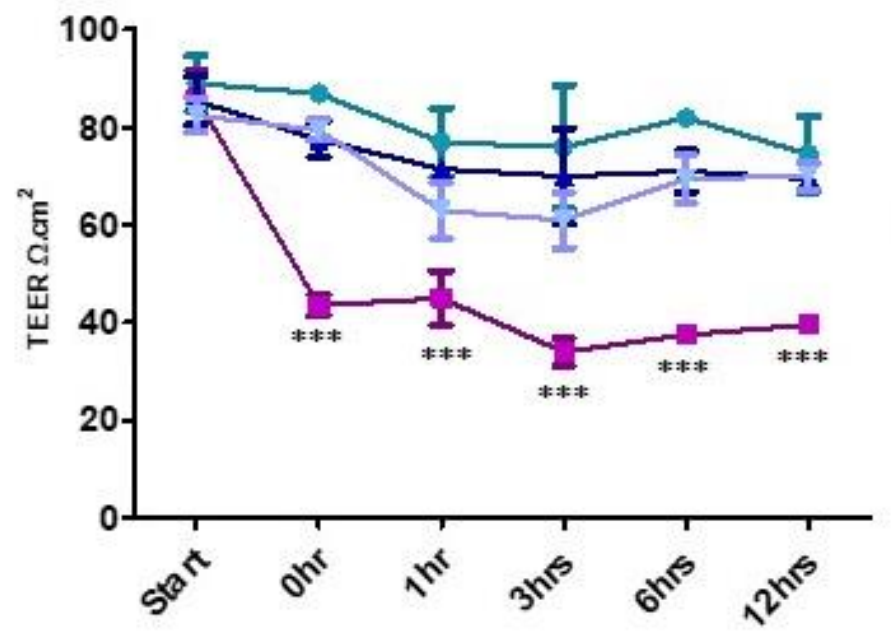

B

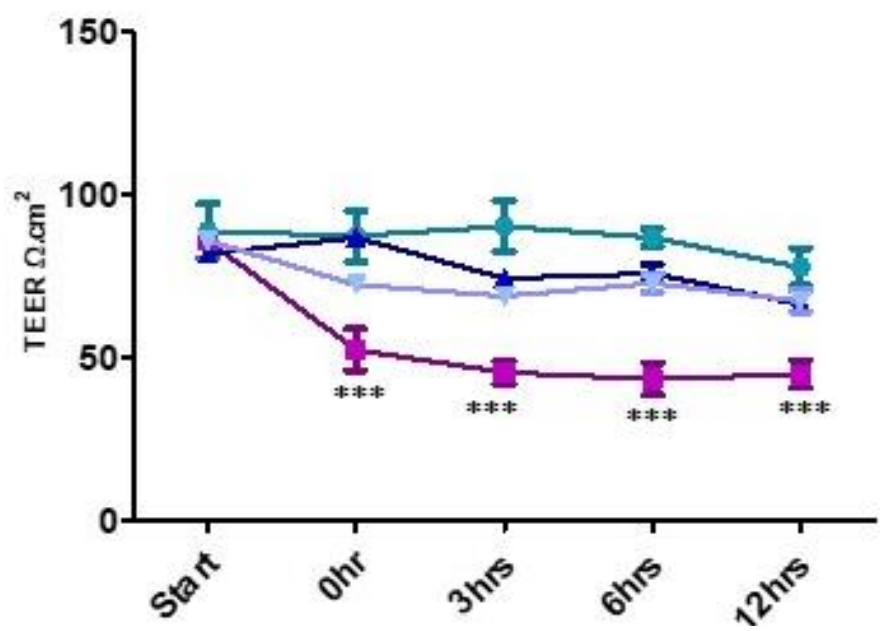

- DMSO

$\rightarrow$ Tnf- $\alpha$

+ RVX208

$\rightarrow$ RVX208+Tnf- $\alpha$

Figure 3.9. BRD4 inhibition, protects monolayer integrity in HUVECs. HUVECs were grown on $0.45 \mu \mathrm{M}$ transwell membrane for 5 days to establish the monolayer Line graphs showing Transepithelial/endothelial electrical resistance (TEER) measured in HUVEC monolayers treated with DMSO, TNF- $\alpha-0$ ly, RVX208-only and RVX208+TNF- $\alpha$ (A), and with DMSO, TNF- $\alpha$-only, JQ1-only and JQ1+TNF- $\alpha(B)$. Values are mean \pm SEM of three biological replicates and asterisks indicate statistical significance of ${ }^{* * *} p<0.001$.

Next, we wanted to investigate the mechanism through which BRD4 inhibition is preserving the monolayer integrity. Here we would like to highlight that our group has previously shown that increased monolayer permeability is associated with Midkine/PI3K pathway in human epithelial colorectal adenocarcinoma cells (Caco-2 cells) (Khan et al., 2017). To evaluate whether the same mechanism/pathway is responsible for the loss of monolayer integrity in HUVECs, we decided first to analyse mRNA expression of these genes during inflammation. HUVECs were grown for 4-5 days to attain monolayer and then were treated 
with TNF- $\alpha$ for 2 and $12 \mathrm{hrs}$. Induced mRNA expression levels of pro-inflammatory gene (SELE) in TNF- $\alpha$ treated monolayers confirmed the inflammatory phenotype as compared to non-treated control monolayers (Fig. 3.10A). Then the expression of p38MAP Kinase (p38MAPK) and MIDKINE was measured (Fig. 3.10B). As our data indicated higher expression of $p 38 M A P K$ and MIDKINE in TNF- $\alpha$ treated monolayers, we decided to explore the role of MIDKINE, which is an upstream regulator of P38MAPK, in conjunction with BRD4 in our experimental setup.

A

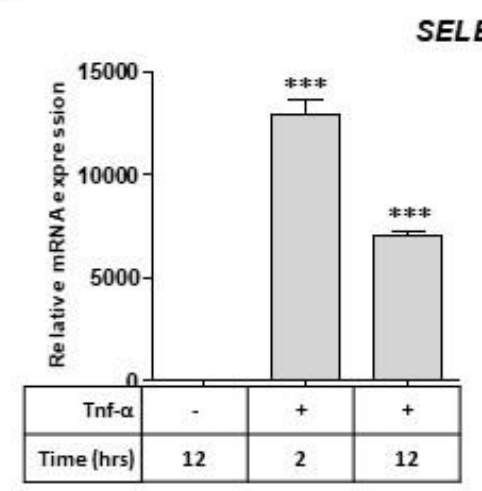

B

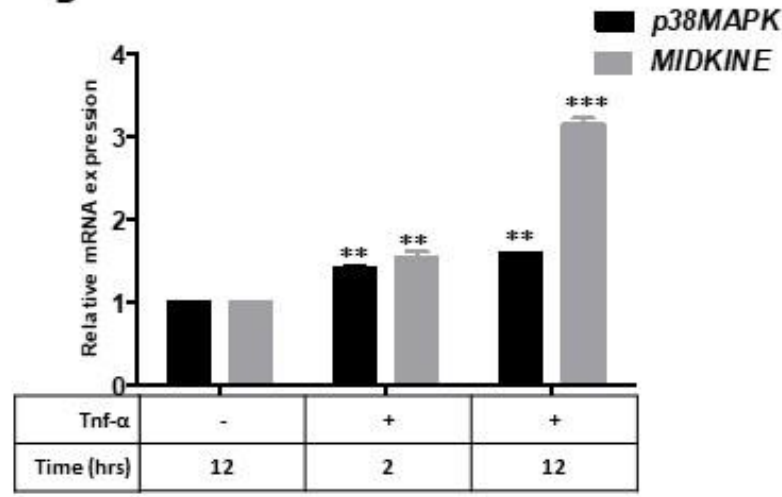

Figure 3.10. Induction of MIDKINE and P38MAPK expression in HUVECs: A, Bar graph showing mRNA levels of SELE after normalisation to house-keeping gene GAPDH in Control (non-treated) and in TNF- $\alpha$ treated (2hrs and $12 \mathrm{hrs})$ monolayers. B, Bar graph showing mRNA levels of P38MAPK and MIDKINE after normalisation to house-keeping gene GAPDH in non-treated control and in TNF- $\alpha$ treated ( $2 \mathrm{hrs}$ and $12 \mathrm{hrs}$ ) monolayers. Values are mean \pm SEM of two biological replicates and asterisks indicate statistical significance of ${ }^{* \star *} p<0.001$.

\subsubsection{TNF- $\alpha$ induces the expression of Midkine}

Endothelium releases a basal level of Midkine, a cytokine, in normal healthy state, where it is known to play a role in angiogenesis, apoptosis, growth and repair of damaged tissues (Kadomatsu et al., 2014; Salaru et al., 2016). Increased expression of Midkine has been reported in inflammatory kidney disease and atherosclerosis (Kadomatsu et al., 2014; Salaru et al., 2013; Salaru et al., 2016). Hence, we decided to check Midkine expression in HUVEC monolayers treated with $20 \mathrm{ng} / \mathrm{ml}$ TNF- $\alpha$ for 6-, 12-, 24-, 48-, and 72- hrs. The mRNA expression of MIDKINE was significantly high after 6 hrs of TNF- $\alpha$ treatment and it steadily increased until $72 \mathrm{hrs}$ of treatment (Fig. 3.11A).

Western blot analysis on total protein extracts of above treatments failed to detect the band corresponding to MIDKINE (Fig. 3.11B). Midkine is released from cell and acts as a signalling molecule, so we checked the cell culture medium supernatants of HUVEC 
monolayer for MIDKINE protein. This analysis indicated that Midkine protein secretion was significantly high in HUVECs treated with TNF-a for 48 hrs-72 hrs (Fig. 3.11C).

A

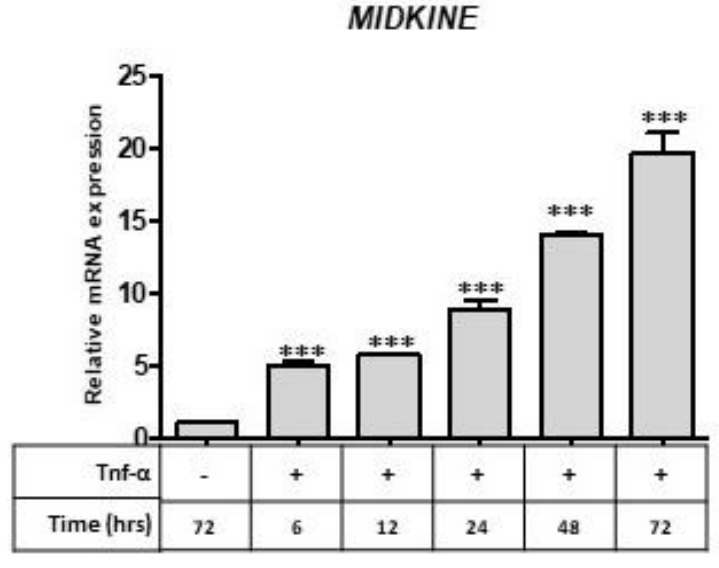

B

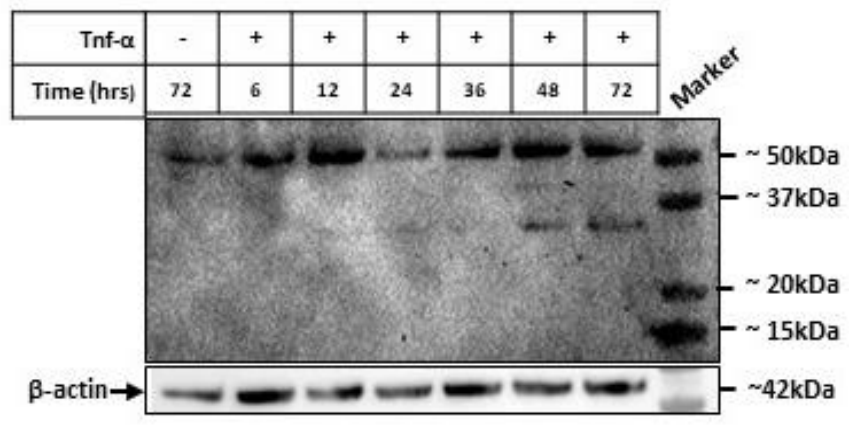

C

\begin{tabular}{r|r|r|r|r|r|r|r|}
\hline Tnf- $\alpha$ & - & + & + & + & + & + & + \\
\hline Time (hrs) & 72 & 6 & 12 & 24 & 36 & 48 & 72 \\
MIDKINE $\rightarrow$ & & & \\
Unknown $\rightarrow$ &
\end{tabular}

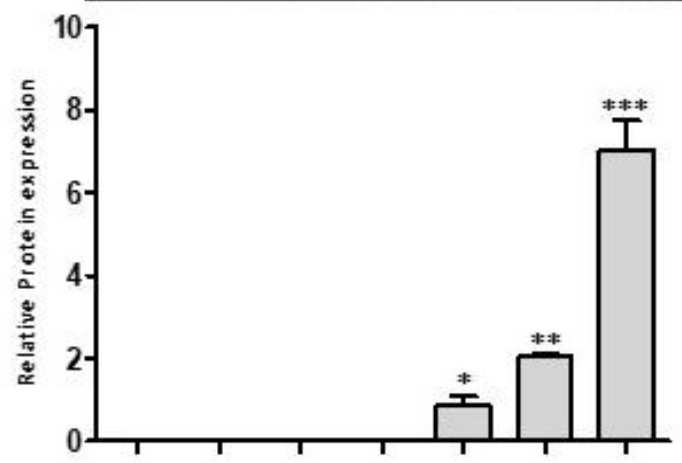

Figure 3.11. Midkine expression in HUVEC monolayer during TNF- $\alpha$ treatment: $A$, Bar graph showing relative mRNA levels of MIDKINE after normalization to house-keeping gene, GAPDH, in control and after different times of TNF- $\alpha$ treatments (6-, 12-, 24-, 48- and 72-hrs). B, Western Blot showing MIDKINE and $\beta$-actin expression in control and TNFa treatments (6-, 12-, 24-, 48- and 72-hrs). C, Western blot image (upper panel) and the corresponding relative quantification bar graph showing the protein expression of MIDKINE secretion (lower panel) in control and TNF-a treatments (6-, 12-, 24-, 36-, 48-, and 72hrs). Ponceau staining of the Western blot membrane showing a band of unknown identity at $\sim 55 \mathrm{kDa}$ as a loading control. Values are mean \pm SEM of three biological replicates and asterisks indicate statistical significance of ${ }^{* *} p<0.001$ and ${ }^{* *} p<0.01$.

This finding together with our previous observation that Midkine disrupts tight junctions and monolayer integrity in Caco-2 monolayer (Khan et al., 2017), made us to conclude that 
Midkine could be the reason for disruption of tight junctions in HUVEC monolayers, as well. Hence, it would be interesting to analyse the effect of BRD4 inhibition on MIDKINE expression and the monolayer integrity properties of HUVECs.

\subsubsection{BRD4 inhibition reduces the expression of Midkine}

To study the effect of BRD4 inhibition on Midkine expression, HUVEC monolayers were pre-treated either with or without RVX208 or JQ1 for 12 hrs before treating them with TNFa for 6-, 12-, 24-, 36-, 48- and 72-hrs. RNA expression analysis showed significantly reduced expression of MIDKINE in monolayers pre-treated with Inhibitor (RVX208 or JQ1) followed by TNF- $\alpha$ treatment compared to TNF- $\alpha$-only treated monolayers (Fig. 3.12A and $B$ respectively).

A

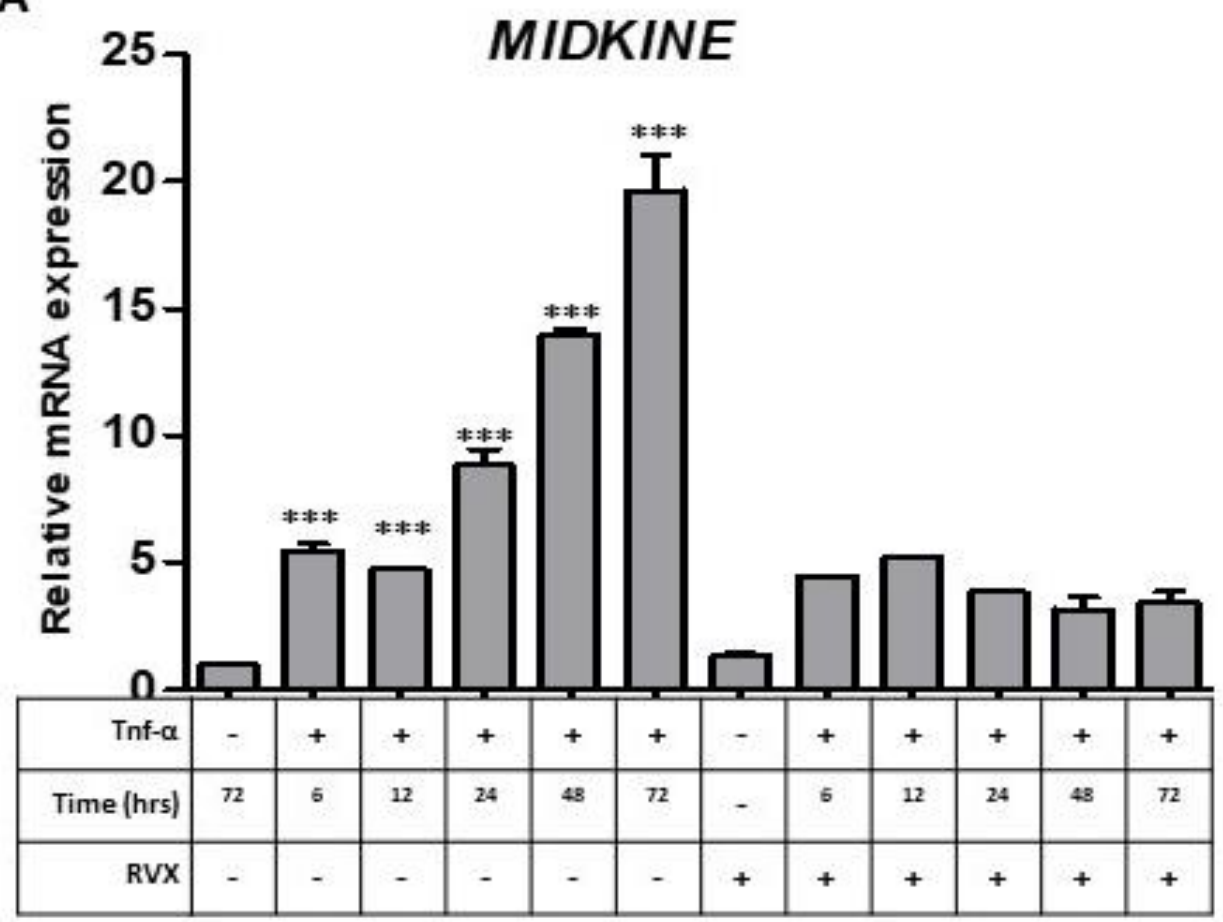


B MIDKINE

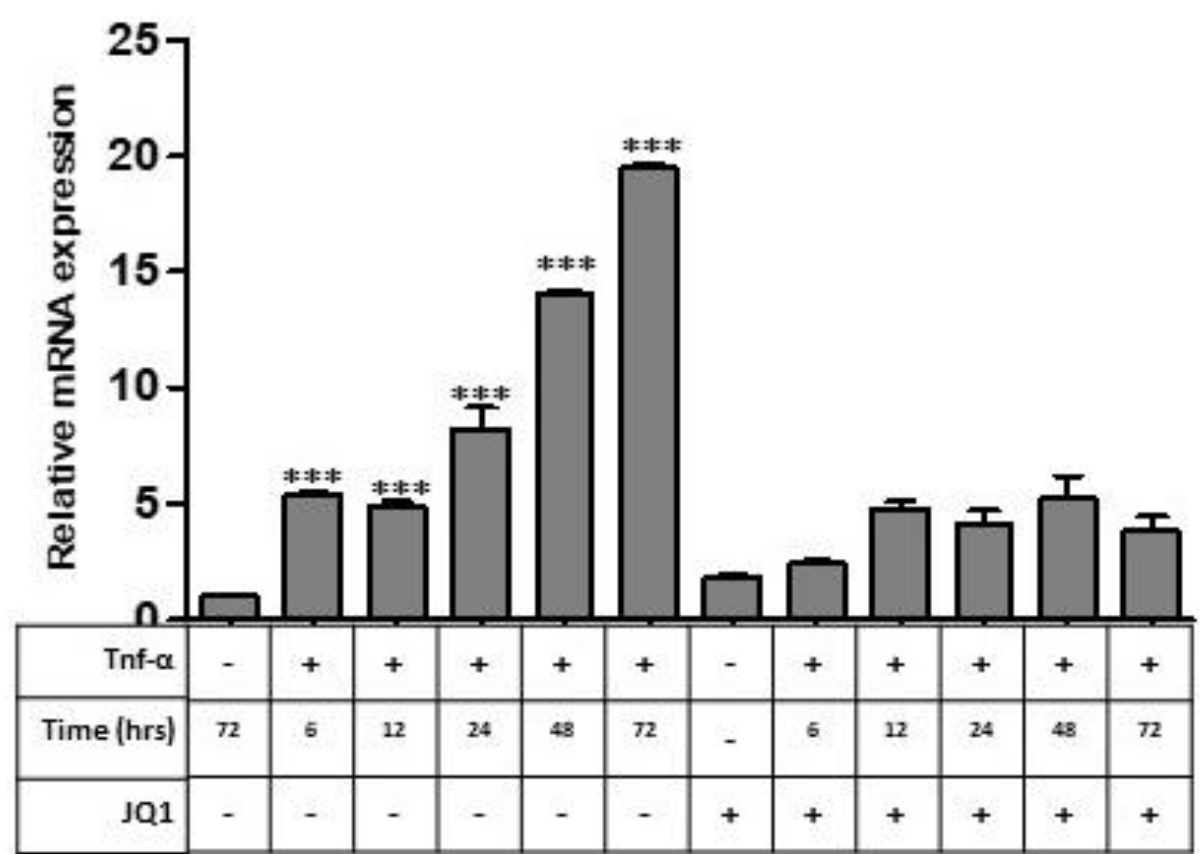

C

\begin{tabular}{|c|c|c|c|c|c|c|c|c|c|}
\hline Tnf- $\alpha$ & - & + & + & + & + & + & + & + & + \\
\hline Time (hrs) & 72 & 6 & 12 & 36 & 48 & 72 & 48 & 72 & 72 \\
\hline $\mathrm{RVX} / \mathrm{JQ} 1$ & - & - & - & - & - & - & $\mathrm{RVX}$ & $\mathrm{R} V \mathrm{X}$ & JQ1 \\
\hline & 2 & & A. & the & $=$ & $=$ & monet & - & $y^{2} 8$ \\
\hline
\end{tabular}

Figure 3.12. Midkine inhibition in HUVECs with BRD4 inhibitors A, Bar graph showing relative mRNA levels of MIDKINE after normalization to house-keeping gene GAPDH, in control and TNF- $\alpha$ treatments (6-, 12-, 24-, 48- and 72hrs), RVX 208 treatment and pre-treatment with RVX208 followed by TNF- $\alpha$ (6-, 12-, 24-, 36-, 48-, and 72-hrs). B, Bar graph showing relative mRNA levels of MIDKINE after normalization to house-keeping gene GAPDH, in control and TNFa treatments (6-, 12-, 24-, 48- and 72-hrs), JQ1 treatment and pre-treatment with JQ1 followed by TNF-a (6-, 12-, 24-, 36-, 48-, and 72-hrs) .C, Western blot image showing the protein expression of MIDKINE secretion (upper panel) in control, TNF- $\alpha$ treatments (36-, 48-, and 72-hrs), Pre-treatment with RVX208 or JQ1 followed by 48- and 72-hrs of TNFa treatment. Ponceau staining (lower panel) of the Western blot membrane showing a band of unknown identity at $\sim 55 \mathrm{kDa}$ as a loading control. Values are mean \pm SEM of three biological replicates and asterisks indicate statistical significance of ${ }^{* * *} p<0.001,{ }^{* *} p<0.01$ and ${ }^{*} p<0.05$.

Next, we checked the supernatants of HUVEC monolayer cultures for secreted Midkine protein. Monolayers were treated either with RVX208 or JQ1 or were treated with RVX208 or JQ1 followed by TNF- $\alpha$ treatment for 48 and 72 hrs. Protein secretion of MIDKINE was significantly reduced in cells pre-treated with BRD4 inhibitor followed by TNF- $\alpha$ treatment, as compared to TNF- $\alpha$-only treatment (Fig. 3.12C). Our results indicate that Midkine 
expression and secretion was significantly high in response to TNF- $\alpha$ treatment. However, BRD4 inhibition via JQ1 or RVX208, decreases the expression of Midkine during TNF- $\alpha$ treatment. These results indicate that MIDKINE might be another target of BRD4 during inflammatory response in HUVECs and inhibition of BRD4 results in decreased expression of MIDKINE.

Collectively, we can conclude that BRD4 inhibition not only alleviates the inflammatory response in HUVECs but also preserves endothelial monolayer integrity via reducing the Midkine expression.

\subsection{Expression of PTP1B increases in ECs during inflammation}

Protein Tyrosine Phosphatase 1B (PTP1B) is another protein which has higher expression during inflammation (Zabolotny et al., 2008). And enhanced expression of PTP1B in ECs is associated with reduced angiogenesis and reduced expression of endothelial specific nitric oxide synthase (eNOS) (Besnier et al., 2014; Lanahan et al., 2014). Then we want to analyse whether PTP1B is highly expressed also in our in-vitro inflammatory model. For this reason, we treated the HUVECs with TNF- $\alpha$ for 2 hrs and analysed the mRNA level of PTP1B. We found no significant expression difference of PTP1B in control and TNF- $\alpha$ treated cells. So, then we treated HUVECs with TNF- $\alpha$ for various time points $(6-, 12-$, and 24-hrs) and found significant up-regulation of PTP1B mRNA levels already by 6 hrs of treatment, which is further increased by $24 \mathrm{hrs}$ (Fig. $3.13 \mathrm{~A}$ ).

Next, we performed the western blot analysis using anti-PTP1B antibody and found significantly increase in expression of PTP1B protein only after $24 \mathrm{hrs}$ of TNF-a treatment (Fig. 3.13B). These results highlight that PTP1B is up-regulated during inflammatory response in ECs. 
A

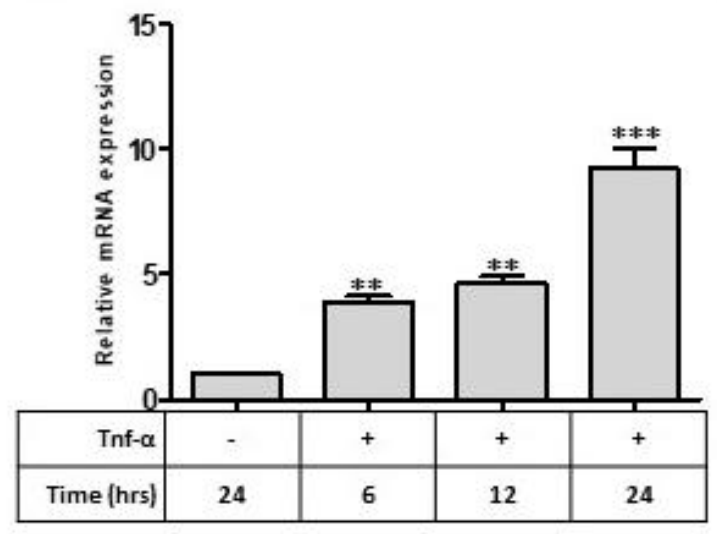

B
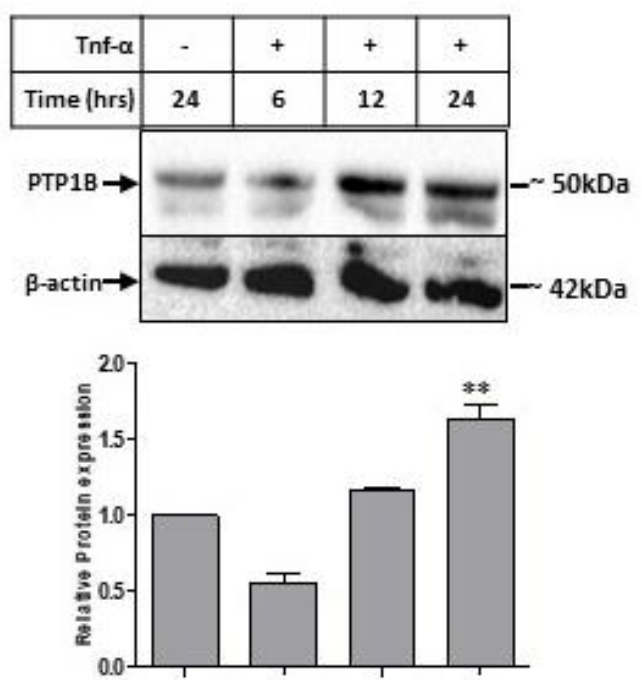

Figure 3.13. Induction of PTP1B expression in HUVECs. A, Bar graph showing relative mRNA levels of PTP1B after normalization to house-keeping gene GAPDH in control, and TNF- $\alpha$ treatments (6-, 12- and 24-hrs). B, Western blot image (upper panel) and the corresponding relative quantification bar graph (lower panel) showing the protein expression of PTP1B in control and TNF- $\alpha$ treated (6-, 12- and 24-hrs). Immunoblotting for $\beta$-actin was used as a loading control. The band intensities of Midkine were normalized to $\beta$-actin. Values are mean \pm SEM of two biological replicates and asterisks indicate statistical significance of ${ }^{* * *} p<0.001$ and ${ }^{* *} p<0.01$

In this context it is interesting to note that deletion or pharmacological inhibition of PTP1B in mice protects them against diabetes and obesity (Ali et al., 2009) and hypertension (Bruder-Nascimento et al., 2015). It has been reported that PTP1B deletion reduces endothelial dysfunction by improving Nitric oxide (NO) production, improves cardiac dysfunction and cardiac inflammation (Coquerel et al., 2014). Importantly endothelial specific PTP1B deletion in mice (Endo.Ptp 1b.KO) protected them against induced cardiac hypertrophy and fibrosis in TAC model (Gogiraju et al., 2016). This finding prompted us to analyse the global proteome of heart tissues derived from Endo.Ptp1b.KO to identify possible targets/pathways of Ptp1b, which are responsible for the observed rescue.

\subsection{Global Proteome analysis}

In order to achieve a comparative global proteome profiling between Endo.Ptp1b.KO and Wild type (Endo.Ptp1b.WT) mouse hearts, the strategy illustrated in figure 3.11 was employed. Briefly, mice with loxP flanked Ptp $1 b$ allele $\left(P t p 1 b^{f / f f}\right)$ were bred either with Wild type mice or with transgenic mice expressing a fusion protein of Cre recombinase-estrogen receptor under the control of endothelial receptor tyrosine kinase promoter 
(Tie2.ERT2.Cre). The off-spring were then fed with Tamoxifen diet to induce Cre recombinase activity. Afterwards, 10-12 weeks-old females WT and KO females were subjected to TAC or SHAM surgery (Fig. 3.14). In TAC group, a band is placed around the aorta of mice to induce pressure on heart, while SHAM mice (an experimental control) group also underwent surgery although aortic arch was exposed but no band was placed around the aorta. This strategy gave rise to four groups of animals namely: 1. Wild-type SHAM (WT-SHAM), 2. Wild-type TAC (WT-TAC), 3. Endothelial-specific Ptp1b knockout SHAM (KO-SHAM), and 4, Endothelial-specific Ptp1b knockout TAC (KO-TAC). After 20 weeks of TAC/SHAM surgery, five independent animals were taken from each group as biological replicates and whole heart tissue lysates were prepared in the presence of protease and phosphatase inhibitors (Fig. 3.14). Afterwards, $50 \mu \mathrm{g}$ of proteins were tryptic digested and the resulting peptides were subjected to liquid chromatography-tandem mass spectrometry (LC-MS/MS) analysis.

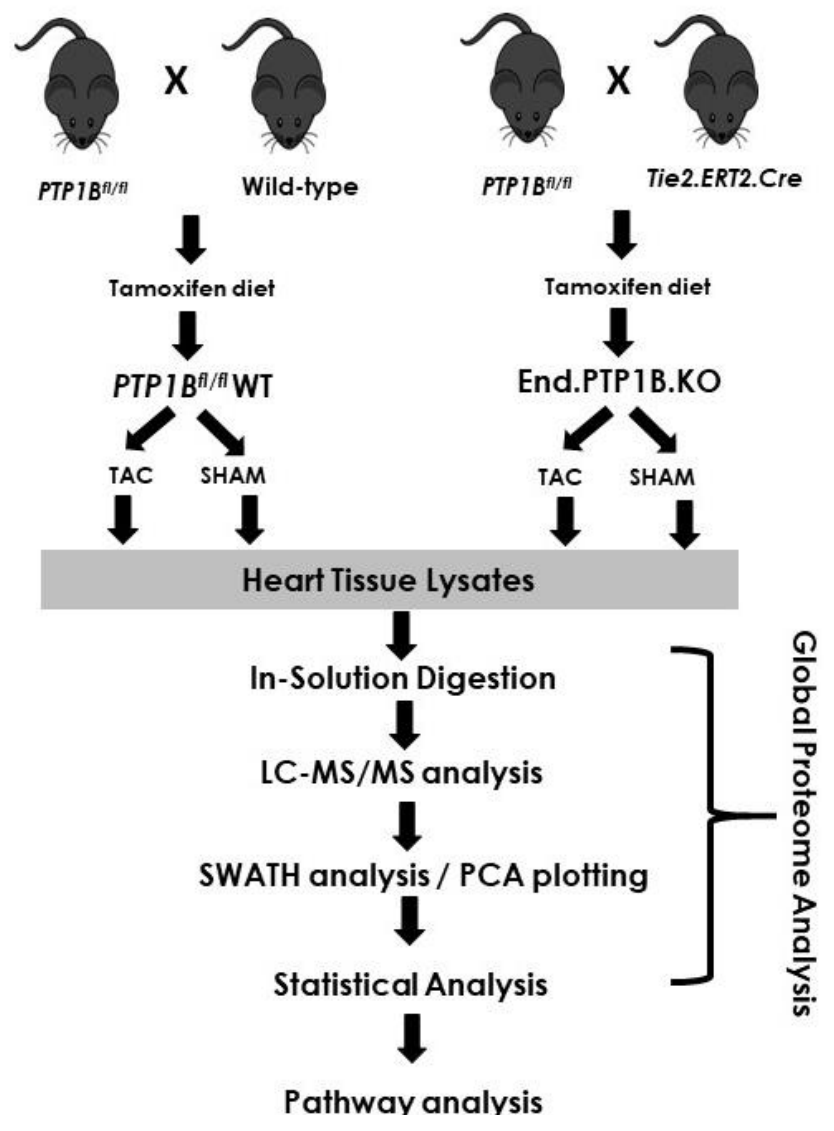

Fig. 3.14. Schematic illustration of strategy for global proteome analysis. Ptp $1 b^{A / f l}$ mice were either crossed with Wild type or with transgenic Tie2.ERT2. Cre mice and the resulting off-spring (6-week-old) were fed with tamoxifen diet for other 6-weeks to induce Cre-recombinase activity and to obtain Endo.Ptp1b.WT or Endo.Ptp1b.KO mice, respectively. Both animal groups underwent the TAC or SHAM surgery and gave rise to four groups: WT-TAC, WT-SHAM, KO-SHAM and KO-TAC. Total heart proteins were tryptic digested and identified by LC-MS/MS analysis and quantified by SWATH 
analysis. Then Perseus based statistical analysis was employed to identify the significant proteins. STRING pathway analysis was performed to identify the sub-cellular localization of proteins for better understanding of their functions.

Following the data acquisition, we performed quantitative proteome analysis by using Sequential Window Acquisition of all THeoretical fragment ion spectra (SWATH) in data independent acquisition (DIA) mode. Overall, we identified a total of 899 common proteins in all four groups and quantified 697 proteins with 1\% False Discovery Rate (FDR). Then, we analysed all 697 proteins by using directed Principal Component Analysis (PCA) to get an overview of variability in our data. We observed four clusters representing four analysed groups with all the biological and technical replicates falling into their respective clusters (Fig. 3.15). WT-SHAM and KO-SHAM were more closely clustered to each other, indicating less variability among the SHAM groups. Interestingly, TAC groups (KO-TAC and WT TAC) represented two distinct clusters, highlighting that the rescue of cardiac overload has a different proteome (Fig. 3.15).

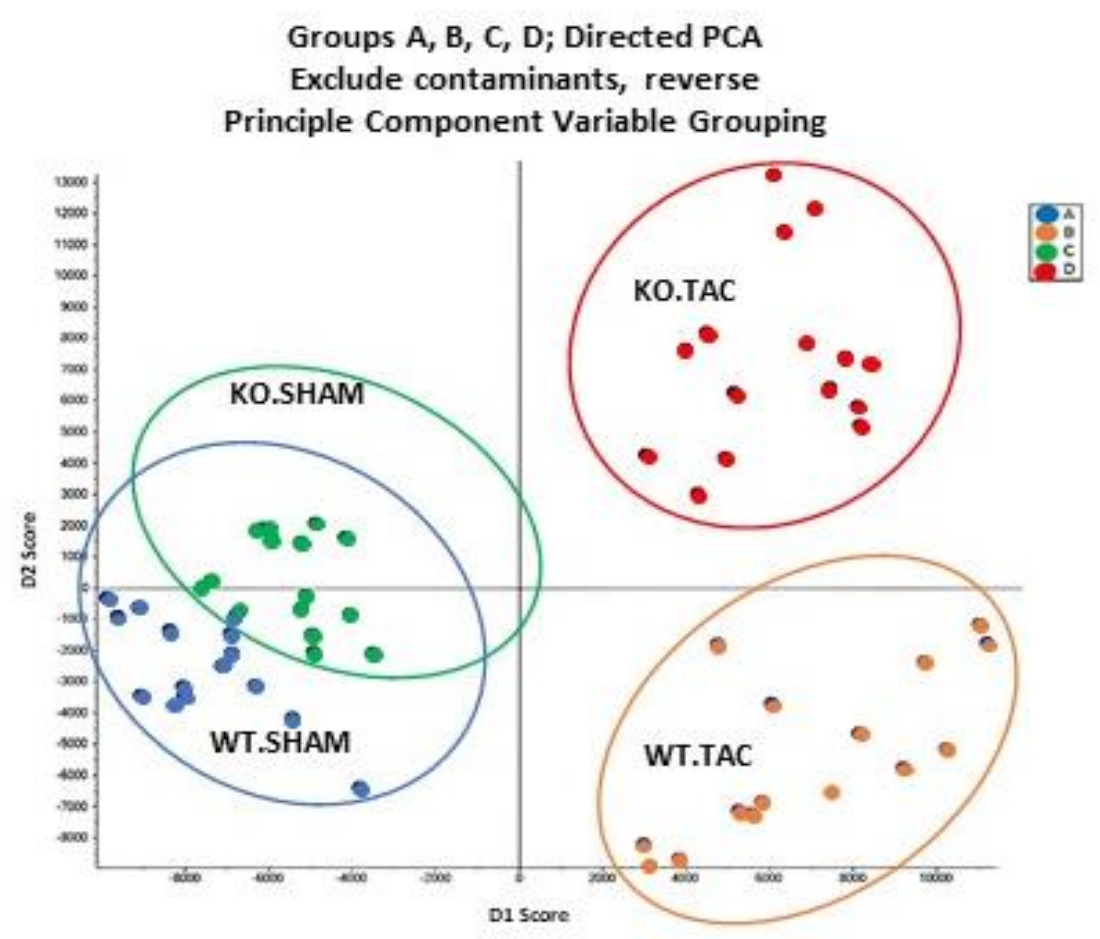

Fig. 3.15. Global proteome analysis: PCA analysis based on whole-proteome levels, which confirmed the presence of the 4 different groups. Different groups were assigned to proteins on basis of differential expression Blue colour represents WT-SHAM group, Orange colour represents WT-TAC, Green colour represents KO-SHAM and Red colour represents KO-TAC group. 


\subsubsection{Statistical Analysis using Perseus Software}

After-knowing that KO-TAC and WT-TAC mice had a different proteome compared to SHAM groups, we decided to perform statistical analysis using Perseus software to identify proteins that have significant expression differences. For this reason, we compared the following groups:

Group A: WT-SHAM and KO-SHAM Group B: WT-SHAM and WT-TAC

Group C: KO-SHAM and KO-TAC Group D: WT-TAC and KO-TAC

Next, we calculated $p$-values for all four groups followed by fold change calculation. We considered proteins with $p$ values $<0.05$ and fold change cut-off value of 1.5 as significantly different and used them for further analysis. In Group A, we found 8 proteins to be upregulated, while 58 proteins to be down-regulated in KO-SHAM as compared to WT-SHAM (Table 3.1, 3.2, and Fig. 3.16) In Group B, 60 and 70 proteins were up- and downregulated, respectively, (Table 3.3, 3.4, and Fig. 3.16) in WT-TAC as compared to WTSHAM. In Group C, 150 proteins were up-regulated while 53 proteins were down-regulated in KO-TAC as compared to KO-SHAM, (Tables 3.5, 3.6, and Fig. 3.16). In Group D: 43 proteins were up-regulated while 36 were down-regulated in KO-TAC as WT-TAC (Table 3.7, 3.8, and Fig. 3.16). 


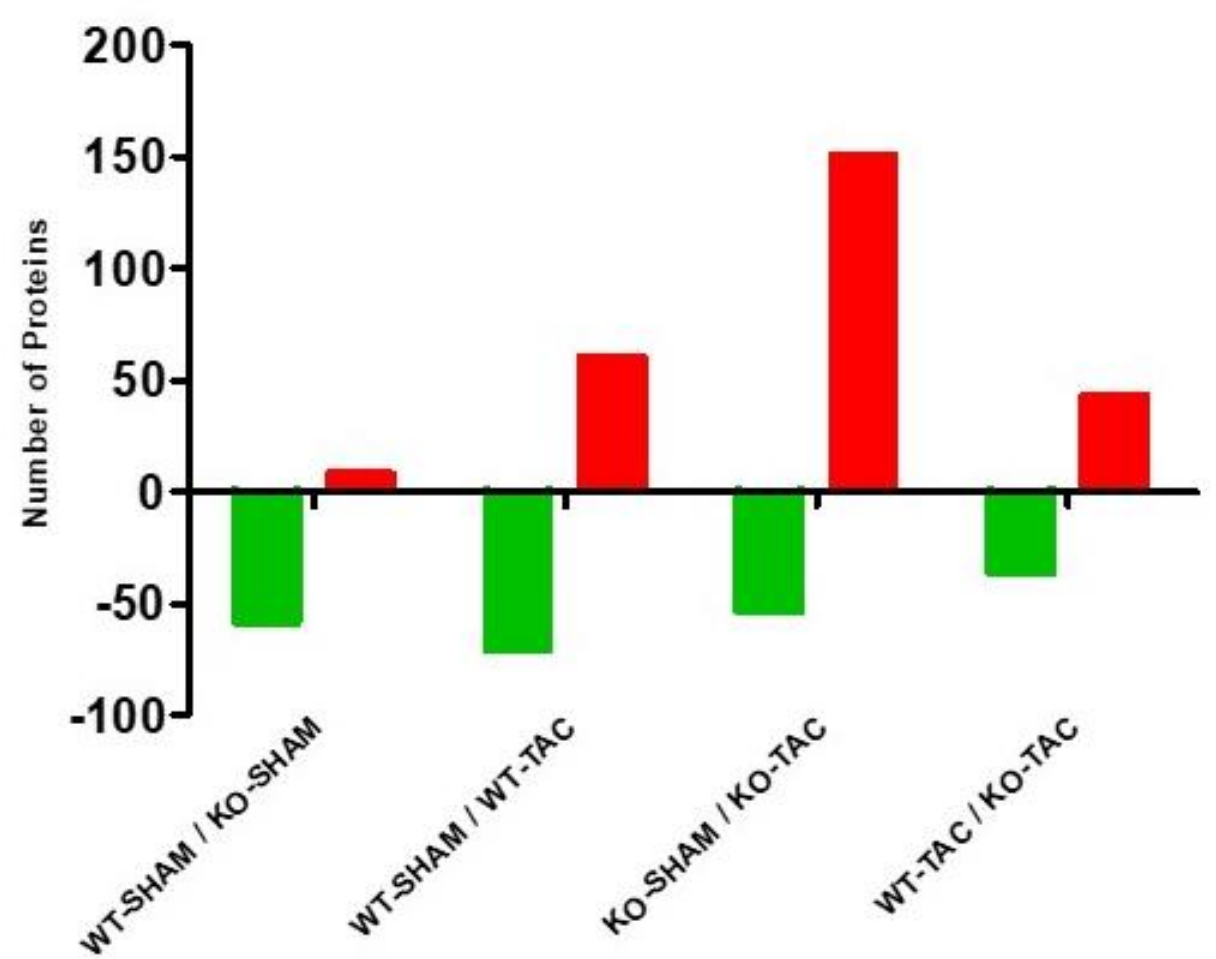

Figure 3.16. Statistical summary of Global proteome analysis: Bar graph indicating number of up-regulated (red) and down-regulated (green) proteins in all four groups. The number of proteins in bar graph denote those that reached statistical significance using Welch's T-test.

Next, we converted the p-values into -log10 (p-values) and fold change was converted in $\log 2$ (log2 FC) and volcano plots were plotted using - $\log 10$ of $p$-values against $\log 2$ of fold change (Fig. 3.17A-D).

\section{Table 3.1}

\begin{tabular}{|l|l|l|}
\hline \multicolumn{2}{|c|}{ Group A: Up-regulated Proteins in KO-SHAM vs WT-SHAM } \\
\hline Uniprot Accession & Protein & Fold Change \\
\hline NDUA3_MOUSE & NADH dehydrogenase 1 alpha subcomplex subunit 3 & 9.261803 \\
\hline CXA1_MOUSE & Gap junction alpha-1 protein & 2.333574 \\
\hline 2AAA_MOUSE & $\begin{array}{l}\text { Serine/threonine-protein phosphatase 2A 65 kDa regulatory } \\
\text { subunit A alpha isoform }\end{array}$ & 2.271057 \\
\hline Q7JCZ3_MOUSE & Cytochrome b & 2.19333 \\
\hline A0A0G2JH00_MOUSE & Protein Igkv4-61 & 2.1896 \\
\hline MYL6_MOUSE & Myosin light polypeptide 6 & 1.99142 \\
\hline
\end{tabular}




\begin{tabular}{|l|l|l|}
\hline NDUA7_MOUSE & NADH dehydrogenase 1 alpha subcomplex subunit 7 & 1.679112 \\
\hline ACADS_MOUSE & Short-chain specific acyl-CoA dehydrogenase, mitochondrial & 1.588391 \\
\hline
\end{tabular}

\section{Table 3.2}

\begin{tabular}{|c|c|c|}
\hline \multicolumn{3}{|c|}{ Group A: Down-regulated Proteins in KO-SHAM vs WT-SHAM } \\
\hline Uniprot Accession & Protein & Fold Change \\
\hline SPA3K_MOUSE & Serine protease inhibitor $\mathrm{A} 3 \mathrm{~K}$ & 5.724768 \\
\hline Q3UER8_MOUSE & Fibrinogen gamma chain & 5.59055 \\
\hline Q58E64_MOUSE & Elongation factor 1-alpha & 4.41397 \\
\hline Q9CPX4_MOUSE & Ferritin & 4.184854 \\
\hline Q52L87_MOUSE & Tubulin alpha-1C chain & 3.284082 \\
\hline Q58E35_MOUSE & $60 \mathrm{~S}$ acidic ribosomal protein $\mathrm{P} 1$ & 3.249365 \\
\hline E9Q5L2_MOUSE & Inter alpha-trypsin inhibitor, heavy chain 4 & 3.172823 \\
\hline Q149V4_MOUSE & Histone $\mathrm{H} 2 \mathrm{~A}$ & 2.851051 \\
\hline AN32A_MOUSE & Acidic leucine-rich nuclear phosphoprotein 32 family member $\mathrm{A}$ & 2.690346 \\
\hline D3Z576_MOUSE & Filamin-C & 2.55819 \\
\hline FIBA_MOUSE & Fibrinogen alpha chain & 2.505103 \\
\hline COF1_MOUSE & Cofilin-1 & 2.465072 \\
\hline Q5DQJ3_MOUSE & $\begin{array}{l}\text { Capping protein (Actin filament) muscle Z-line, alpha 2, isoform } \\
\text { CRA_c }\end{array}$ & 2.453384 \\
\hline GNAS1_MOUSE & $\begin{array}{l}\text { Guanine nucleotide-binding protein G(s) subunit alpha isoforms } \\
\text { XLas }\end{array}$ & 2.332206 \\
\hline A2AFJ1_MOUSE & Histone-binding protein RBBP7 & 2.283441 \\
\hline Q546K1_MOUSE & Cytoglobin & 2.23178 \\
\hline RTN2_MOUSE & Isoform 2 of Reticulon-2 & 2.221354 \\
\hline PP1B_MOUSE & $\begin{array}{l}\text { Serine/threonine-protein phosphatase PP1-beta catalytic } \\
\text { subunit }\end{array}$ & 2.218909 \\
\hline Q8BVQ9_MOUSE & $26 S$ protease regulatory subunit 7 & 2.188379 \\
\hline SDCG8_MOUSE & Serologically defined colon cancer antigen 8 homolog & 2.157165 \\
\hline RS9_MOUSE & 40S ribosomal protein S9 & 2.09963 \\
\hline A4FUS1_MOUSE & $40 S$ ribosomal protein $\mathrm{S} 16$ & 2.052678 \\
\hline Q8C5G6_MOUSE & Toll-interacting protein & 2.029818 \\
\hline Q5SUC3_MOUSE & Calnexin & 2.009529 \\
\hline A0A075B6A0_MOUSE & $\lg$ mu chain $\mathrm{C}$ region & 2.008865 \\
\hline S4R1E5_MOUSE & Glutathione peroxidase & 2.006824 \\
\hline ANXA3_MOUSE & Annexin A3 & 1.970541 \\
\hline TERA_MOUSE & Transitional endoplasmic reticulum ATPase & 1.908613 \\
\hline Q499X4_MOUSE & Synaptic vesicle membrane protein VAT-1 homolog & 1.900839 \\
\hline A3KGQ6_MOUSE & Actin-related protein $2 / 3$ complex subunit 5 & 1.857499 \\
\hline Q3U4U6_MOUSE & T-complex protein 1 subunit gamma & 1.843001 \\
\hline Q792A4_MOUSE & Cox7ah & 1.834669 \\
\hline A0A087WS46_MOUSE & Elongation factor 1-beta & 1.830365 \\
\hline
\end{tabular}




\begin{tabular}{|c|c|c|}
\hline HSPB2_MOUSE & Heat shock protein beta-2 & 1.825187 \\
\hline Q564E8_MOUSE & $60 S$ ribosomal protein $\mathrm{L} 4$ & 1.806892 \\
\hline Q545X8_MOUSE & $40 S$ ribosomal protein S4 & 1.798365 \\
\hline DNJB4_MOUSE & DNA homolog subfamily B member 4 & 1.794935 \\
\hline TM14C_MOUSE & Transmembrane protein $14 \mathrm{C}$ & 1.781072 \\
\hline Q3U367_MOUSE & 4-trimethylaminobutyraldehyde dehydrogenase & 1.756965 \\
\hline GRPE1_MOUSE & GrpE protein homolog 1, mitochondrial & 1.749118 \\
\hline A0A0H2UH17_MOUSE & Ubiquitin associated protein 2-like, isoform CRA_b & 1.72855 \\
\hline Q5M8M8_MOUSE & $60 S$ ribosomal protein L29 & 1.704919 \\
\hline G5E8J6_MOUSE & Histidine rich calcium binding protein, isoform CRA_a & 1.694952 \\
\hline Q543B9_MOUSE & Prolyl endopeptidase & 1.684433 \\
\hline CAND1_MOUSE & Cullin-associated NEDD8-dissociated protein 1 & 1.678813 \\
\hline Q5M9J2_MOUSE & Histidine triad nucleotide binding protein 2 & 1.663793 \\
\hline CD47_MOUSE & Leukocyte surface antigen CD47 & 1.662129 \\
\hline RL27A_MOUSE & $60 S$ ribosomal protein L27a & 1.648814 \\
\hline TNNC1_MOUSE & Troponin C, slow skeletal and cardiac muscles & 1.632444 \\
\hline USMG5_MOUSE & Up-regulated during skeletal muscle growth protein 5 & 1.60865 \\
\hline Q3UCD9_MOUSE & Cathepsin D & 1.604517 \\
\hline A0A0U1RQ27_MOUSE & Methylmalonyl CoA epimerase, isoform CRA_C & 1.573786 \\
\hline B9EHNO_MOUSE & Ubiquitin-activating enzyme $\mathrm{E} 1$, Chr X & 1.573732 \\
\hline Q3TJY2_MOUSE & WD repeat domain 1 & 1.573184 \\
\hline E9Q7N5_MOUSE & Ribosome-releasing factor 2 , mitochondrial & 1.551304 \\
\hline VTDB_MOUSE & Vitamin D-binding protein & 1.543408 \\
\hline GDIR1_MOUSE & Rho GDP-dissociation inhibitor 1 & 1.542594 \\
\hline Q3UL22_MOUSE & Chaperonin subunit 8 (Theta), isoform CRA_a & 1.524054 \\
\hline
\end{tabular}

\section{Table 3.3}

\begin{tabular}{|l|l|l|}
\hline \multicolumn{2}{|c|}{ Group B: Up-regulated Proteins in WT-TAC vS WT-SHAM } \\
\hline Uniprot Accession & Protein & Fold Change \\
\hline B2RXX9_MOUSE & Myosin, heavy polypeptide 7, cardiac muscle, beta & 22.46804 \\
\hline A2AEX8_MOUSE & Four and a half LIM domains 1, isoform CRA_b & 4.475918 \\
\hline Q8BFQ1_MOUSE & Prosaposin & 3.895401 \\
\hline APOA2_MOUSE & Apolipoprotein A-II & 3.506413 \\
\hline Q546K1_MOUSE & Cytoglobin & 3.194436 \\
\hline NDUA3_MOUSE & NADH dehydrogenase 1 alpha subcomplex subunit 3 & 2.944347 \\
\hline D9J302_MOUSE & ENH isoform 1e & 2.922764 \\
\hline TBB5_MOUSE & Tubulin beta & 2.672675 \\
\hline NDUB2_MOUSE & $\begin{array}{l}\text { NADH dehydrogenase 1 beta subcomplex subunit 2, } \\
\text { mitochondrial }\end{array}$ & 2.565567 \\
\hline Q4FJQ6_MOUSE & $\begin{array}{l}\text { Serine (Or cysteine) peptidase inhibitor, clade B, member 6a, } \\
\text { isoform CRA_a }\end{array}$ & 2.548441 \\
\hline H7BX64_MOUSE & Sarcolemmal membrane-associated protein & 2.520141 \\
\hline G5E8T9_MOUSE & Hydroxyacyl glutathione hydrolase & 2.472386 \\
\hline
\end{tabular}




\begin{tabular}{|c|c|c|}
\hline Q7JCZ3_MOUSE & Cytochrome b & 2.44693 \\
\hline E9QLZ9_MOUSE & Protein enabled homolog & 2.410786 \\
\hline G3X9L6_MOUSE & MCG55033 & 2.36428 \\
\hline A0A0A6YWR2_MOUSE & Ig gamma-1 chain $\mathrm{C}$ region secreted form & 2.243095 \\
\hline UGPA_MOUSE & UTP--glucose-1-phosphate uridylyltransferase & 2.227797 \\
\hline TXD17_MOUSE & Thioredoxin domain-containing protein 17 & 2.17689 \\
\hline F6ZFU0_MOUSE & Elongation factor 1-delta & 2.131331 \\
\hline HEMO_MOUSE & Hemopexin & 2.129784 \\
\hline HDHD2_MOUSE & $\begin{array}{l}\text { Haloacid dehalogenase-like hydrolase domain-containing protein } \\
2\end{array}$ & 2.074073 \\
\hline ANXA7_MOUSE & Annexin A7 & 2.073107 \\
\hline Q6YJU1_MOUSE & Fetuin-B & 1.985978 \\
\hline STIP1_MOUSE & Stress-induced-phosphoprotein 1 & 1.984306 \\
\hline CAH1_MOUSE & Carbonic anhydrase 1 & 1.964014 \\
\hline S4R1E5_MOUSE & Glutathione peroxidase & 1.961202 \\
\hline TOM70_MOUSE & Mitochondrial import receptor subunit TOM70 & 1.925854 \\
\hline OBSCN_MOUSE & Obscurin & 1.912945 \\
\hline PSA6_MOUSE & Proteasome subunit alpha type- 6 & 1.904566 \\
\hline F6TCV0_MOUSE & Serine protease HTRA2, mitochondrial & 1.896703 \\
\hline Q3T9Z2_MOUSE & Glyoxylate reductase/hydroxypyruvate reductase & 1.882532 \\
\hline SLIRP_MOUSE & SRA stem-loop-interacting RNA-binding protein, mitochondrial & 1.877301 \\
\hline GCAM_MOUSE & Ig gamma-2A chain $\mathrm{C}$ region, membrane-bound form & 1.873636 \\
\hline TIM13_MOUSE & Mitochondrial import inner membrane translocase subunit Tim13 & 1.869761 \\
\hline Q543M7_MOUSE & Importin subunit alpha & 1.850016 \\
\hline Q545C7_MOUSE & Cysteine and glycine-rich protein 3 & 1.848998 \\
\hline Q542Y0_MOUSE & $\mathrm{NAD}(\mathrm{P}) \mathrm{H}$ dehydrogenase [quinone] 1 & 1.803744 \\
\hline ENOB_MOUSE & Beta-enolase & 1.801966 \\
\hline Z4YK42_MOUSE & NAD kinase 2, mitochondrial & 1.786285 \\
\hline MIRO1_MOUSE & Isoform 4 of Mitochondrial Rho GTPase 1 & 1.779831 \\
\hline F6WYM0_MOUSE & Septin-2 & 1.776692 \\
\hline A0A0G2JH00_MOUSE & Protein Igkv4-61 & 1.770807 \\
\hline HSPB6_MOUSE & Heat shock protein beta- 6 & 1.759373 \\
\hline MYL1_MOUSE & Myosin light chain $1 / 3$, skeletal muscle isoform & 1.757874 \\
\hline E9Q616_MOUSE & Protein Ahnak & 1.751377 \\
\hline OLA1_MOUSE & Obg-like ATPase 1 & 1.74836 \\
\hline PYRD_MOUSE & Dihydroorotate dehydrogenase (quinone), mitochondrial & 1.735936 \\
\hline GPD1L_MOUSE & Isoform 2 of Glycerol-3-phosphate dehydrogenase 1-like protein & 1.702011 \\
\hline A2AIW9_MOUSE & Mitochondrial-processing peptidase subunit alpha & 1.684361 \\
\hline A0A0G2JFH2_MOUSE & Microtubule-associated protein & 1.681806 \\
\hline 2AAA_MOUSE & $\begin{array}{l}\text { Serine/threonine-protein phosphatase } 2 \mathrm{~A} 65 \mathrm{kDa} \text { regulatory } \\
\text { subunit A alpha isoform }\end{array}$ & 1.677898 \\
\hline F2Z456_MOUSE & NADH-cytochrome b5 reductase & 1.657675 \\
\hline PDC6I_MOUSE & Programmed cell death 6-interacting protein & 1.656956 \\
\hline BDH_MOUSE & D-beta-hydroxybutyrate dehydrogenase, mitochondrial & 1.64578 \\
\hline D2KHZ9_MOUSE & Glyceraldehyde-3-phosphate dehydrogenase & 1.633885 \\
\hline
\end{tabular}




\begin{tabular}{|l|l|l|}
\hline Q3TJY2_MOUSE & WD repeat domain 1 & 1.600595 \\
\hline DNJA2_MOUSE & DnaJ homolog subfamily A member 2 & 1.588696 \\
\hline B7ZCI2_MOUSE & Nebulette & 1.584902 \\
\hline F8WIA1_MOUSE & CAP-Gly domain-containing linker protein 1 & 1.575521 \\
\hline E9Q3X0_MOUSE & Major vault protein & 1.567592 \\
\hline LEG1_MOUSE & Galectin-1 & 1.566273 \\
\hline
\end{tabular}

\section{Table 3.4}

\begin{tabular}{|c|c|c|}
\hline \multicolumn{3}{|c|}{ Group B: Down-regulated Proteins in WT-TAC vs WT-SHAM } \\
\hline Uniprot Accession & Protein & Fold Change \\
\hline Q58E35_MOUSE & $60 S$ acidic ribosomal protein $\mathrm{P} 1$ & 7.177066 \\
\hline Q9CPX4_MOUSE & Ferritin & 4.297613 \\
\hline A1BG_MOUSE & Alpha-1B-glycoprotein & 4.065593 \\
\hline Q8BTU6_MOUSE & Eukaryotic initiation factor $4 \mathrm{~A}-\mathrm{II}$ & 3.96271 \\
\hline G5E8J6_MOUSE & Histidine rich calcium binding protein, isoform CRA_a & 3.869917 \\
\hline FRIH_MOUSE & Ferritin heavy chain & 3.731264 \\
\hline Q52L87_MOUSE & Tubulin alpha-1C chain & 3.45146 \\
\hline ABCB8_MOUSE & ATP-binding cassette sub-family B member 8 , mitochondrial & 3.345933 \\
\hline Q3U367_MOUSE & 4-trimethylaminobutyraldehyde dehydrogenase & 3.233596 \\
\hline KV3A4_MOUSE & Ig kappa chain V-III region 50S10.1 & 2.888454 \\
\hline SPA3K_MOUSE & Serine protease inhibitor $\mathrm{A} 3 \mathrm{~K}$ & 2.837102 \\
\hline E9QMK9_MOUSE & Protein 9030617O03Rik & 2.813919 \\
\hline THIM_MOUSE & 3-ketoacyl-CoA thiolase, mitochondrial & 2.793903 \\
\hline B2RUK5_MOUSE & Methylcrotonoyl-CoA carboxylase beta chain, mitochondrial & 2.621667 \\
\hline CES1D_MOUSE & Carboxylesterase 1D & 2.557144 \\
\hline A0A075B6A0_MOUSE & Ig mu chain $\mathrm{C}$ region & 2.486141 \\
\hline RLA2_MOUSE & $60 S$ acidic ribosomal protein $\mathrm{P} 2$ & 2.451661 \\
\hline Q5DQJ3_MOUSE & $\begin{array}{l}\text { Capping protein (Actin filament) muscle Z-line, alpha 2, isoform } \\
\text { CRA_c }\end{array}$ & 2.363354 \\
\hline ECl1_MOUSE & Enoyl-CoA delta isomerase 1, mitochondrial & 2.353803 \\
\hline NDUB4_MOUSE & NADH dehydrogenase 1 beta subcomplex subunit 4 & 2.310692 \\
\hline Q4FK74_MOUSE & ATP synthase subunit delta, mitochondrial & 2.270961 \\
\hline Q0PD66_MOUSE & RAB1B, member RAS oncogene family, isoform CRA_C & 2.267102 \\
\hline CXA1_MOUSE & Gap junction alpha-1 protein & 2.243188 \\
\hline NIT2_MOUSE & Omega-amidase NIT2 & 2.156661 \\
\hline Q32P04_MOUSE & Keratin 5 & 2.149984 \\
\hline A0A0U1RPI1_MOUSE & Phospholemman & 2.125259 \\
\hline Q7JCX7_MOUSE & Cytochrome c oxidase subunit 3 & 2.097943 \\
\hline G3X9J1_MOUSE & Sodium/calcium exchanger 1 & 2.091153 \\
\hline LDHB_MOUSE & L-lactate dehydrogenase $B$ chain & 2.079872 \\
\hline NDUB1_MOUSE & NADH dehydrogenase 1 beta subcomplex subunit 1 & 2.04942 \\
\hline COQ3_MOUSE & Ubiquinone biosynthesis O-methyltransferase, mitochondrial & 2.02944 \\
\hline Q3U7Z6_MOUSE & MCG113582 & 2.011059 \\
\hline
\end{tabular}




\begin{tabular}{|c|c|c|}
\hline LAMA2_MOUSE & Laminin subunit alpha-2 & 1.985002 \\
\hline ADCK3_MOUSE & Atypical kinase ADCK3, mitochondrial & 1.960187 \\
\hline HHATL_MOUSE & Protein-cysteine N-palmitoyltransferase HHAT-like protein & 1.927543 \\
\hline A0A0A0MQ68_MOUSE & Glutaryl-CoA dehydrogenase, mitochondrial & 1.866263 \\
\hline ACOT2_MOUSE & Acyl-coenzyme A thioesterase 2, mitochondrial & 1.865219 \\
\hline TERA_MOUSE & Transitional endoplasmic reticulum ATPase & 1.861359 \\
\hline TACO1_MOUSE & Translational activator of cytochrome $\mathrm{c}$ oxidase 1 & 1.841498 \\
\hline TRAP1_MOUSE & Heat shock protein $75 \mathrm{kDa}$, mitochondrial & 1.810444 \\
\hline CAD13_MOUSE & Cadherin-13 & 1.796888 \\
\hline ECHD2_MOUSE & Enoyl-CoA hydratase domain-containing protein 2 , mitochondrial & 1.775955 \\
\hline HCDH_MOUSE & Hydroxyacyl-coenzyme A dehydrogenase, mitochondrial & 1.769064 \\
\hline PYGM_MOUSE & Glycogen phosphorylase, muscle form & 1.768924 \\
\hline RM12_MOUSE & 39S ribosomal protein L12, mitochondrial & 1.757314 \\
\hline IVD_MOUSE & Isovaleryl-CoA dehydrogenase, mitochondrial & 1.751905 \\
\hline RL14_MOUSE & $60 S$ ribosomal protein $\mathrm{L} 14$ & 1.72281 \\
\hline CD47_MOUSE & Leukocyte surface antigen CD47 & 1.714945 \\
\hline ECHB_MOUSE & Trifunctional enzyme subunit beta, mitochondrial & 1.709272 \\
\hline CAV1_MOUSE & Caveolin-1 & 1.707292 \\
\hline Q80ZZ0_MOUSE & EH domain-containing protein 1 & 1.685678 \\
\hline USMG5_MOUSE & Up-regulated during skeletal muscle growth protein 5 & 1.670951 \\
\hline B2RS41_MOUSE & Aldhehyde dehydrogenase family 5 , subfamily $\mathrm{A} 1$ & 1.670343 \\
\hline TMM65_MOUSE & Transmembrane protein 65 & 1.664084 \\
\hline CACP_MOUSE & Carnitine O-acetyltransferase & 1.659263 \\
\hline NNRE_MOUSE & $\mathrm{NAD}(\mathrm{P}) \mathrm{H}$-hydrate epimerase & 1.647341 \\
\hline GSTK1_MOUSE & Glutathione S-transferase kappa 1 & 1.64678 \\
\hline Q14AZ9_MOUSE & Zinc binding alcohol dehydrogenase, domain containing 2 & 1.633292 \\
\hline CPT2_MOUSE & Carnitine O-palmitoyltransferase 2, mitochondrial & 1.616862 \\
\hline MTFP1_MOUSE & Mitochondrial fission process protein 1 & 1.613282 \\
\hline ACADM_MOUSE & Medium-chain specific acyl-CoA dehydrogenase, mitochondrial & 1.598459 \\
\hline F8WIT2_MOUSE & Annexin & 1.589367 \\
\hline TNNC1_MOUSE & Troponin C, slow skeletal and cardiac muscles & 1.584537 \\
\hline ECHA_MOUSE & Trifunctional enzyme subunit alpha, mitochondrial & 1.576044 \\
\hline Q497X4_MOUSE & Cathepsin R & 1.570785 \\
\hline Q569W4_MOUSE & Acetyl-coenzyme A synthetase & 1.554089 \\
\hline B1AR28_MOUSE & Very long-chain-specific acyl-CoA dehydrogenase, mitochondrial & 1.537874 \\
\hline E9Q800_MOUSE & MICOS complex subunit MIC60 & 1.527675 \\
\hline PDK2_MOUSE & $\begin{array}{l}\text { [Pyruvate dehydrogenase (acetyl-transferring)] kinase isozyme 2, } \\
\text { mitochondrial }\end{array}$ & 1.523358 \\
\hline
\end{tabular}

\section{Table 3.5}

\begin{tabular}{|l|l|l|}
\hline Uniprot Accession & Protein & Fold Change
\end{tabular}




\begin{tabular}{|c|c|c|}
\hline Q546K1_MOUSE & Cytoglobin & 8.179658 \\
\hline B2RXX9_MOUSE & Myosin, heavy polypeptide 7 , cardiac muscle, beta & 7.751299 \\
\hline Q3UER8_MOUSE & Fibrinogen gamma chain & 6.92076 \\
\hline NNTM_MOUSE & $N A D(P)$ transhydrogenase, mitochondrial & 6.404869 \\
\hline Q8C5G6_MOUSE & Toll-interacting protein & 4.803583 \\
\hline S4R1E5_MOUSE & Glutathione peroxidase & 4.76199 \\
\hline RTN2_MOUSE & Isoform 2 of Reticulon-2 & 4.211308 \\
\hline GNAS1_MOUSE & $\begin{array}{l}\text { Guanine nucleotide-binding protein G(s) subunit alpha isoforms } \\
\text { XLas }\end{array}$ & 4.191142 \\
\hline Q58E64_MOUSE & Elongation factor 1 -alpha & 4.157227 \\
\hline Q792A4_MOUSE & Cox7ah & 4.135588 \\
\hline F6TCV0_MOUSE & Serine protease HTRA2, mitochondrial & 4.06327 \\
\hline Q3UP42_MOUSE & Protein S100-A9 & 3.921346 \\
\hline Q542X7_MOUSE & Chaperonin subunit 2 (Beta), isoform CRA_a & 3.866951 \\
\hline Q8BH80_MOUSE & Vesicle-associated membrane protein, associated protein $B$ and $C$ & 3.813203 \\
\hline A3KGQ6_MOUSE & Actin-related protein $2 / 3$ complex subunit 5 & 3.709066 \\
\hline TM14C_MOUSE & Transmembrane protein $14 \mathrm{C}$ & 3.630261 \\
\hline Q54219_MOUSE & $26 \mathrm{~S}$ protease regulatory subunit 4 & 3.286168 \\
\hline PDIA1_MOUSE & Protein disulfide-isomerase & 3.276221 \\
\hline SPA3K_MOUSE & Serine protease inhibitor A3K & 3.256386 \\
\hline Q3UW66_MOUSE & Sulfurtransferase & 3.194263 \\
\hline A2AFJ1_MOUSE & Histone-binding protein RBBP7 & 3.132479 \\
\hline A2AEX8_MOUSE & Four and a half LIM domains 1, isoform CRA_b & 3.085688 \\
\hline E9Q5L2_MOUSE & Inter alpha-trypsin inhibitor, heavy chain 4 & 3.072361 \\
\hline E9Q7N5_MOUSE & Ribosome-releasing factor 2, mitochondrial & 2.989694 \\
\hline D3Z576_MOUSE & Filamin-C & 2.882748 \\
\hline ANXA3_MOUSE & Annexin A3 & 2.876219 \\
\hline TKT_MOUSE & Transketolase & 2.855865 \\
\hline Q4FJQ6_MOUSE & $\begin{array}{l}\text { Serine (Or cysteine) peptidase inhibitor, clade } \mathrm{B}, \text { member } 6 \mathrm{a}, \\
\text { isoform CRA_a }\end{array}$ & 2.822981 \\
\hline Q6YJU1_MOUSE & Fetuin-B & 2.779361 \\
\hline PYRD_MOUSE & Dihydroorotate dehydrogenase (quinone), mitochondrial & 2.772408 \\
\hline DNJB4_MOUSE & DnaJ homolog subfamily B member 4 & 2.739464 \\
\hline HSPB2_MOUSE & Heat shock protein beta-2 & 2.739235 \\
\hline OLA1_MOUSE & Obg-like ATPase 1 & 2.72664 \\
\hline A2AIW9_MOUSE & Mitochondrial-processing peptidase subunit alpha & 2.687923 \\
\hline Q5SUC3_MOUSE & Calnexin & 2.665787 \\
\hline B1AX58_MOUSE & Plastin-3 & 2.637293 \\
\hline Q3TJY2_MOUSE & WD repeat domain 1 & 2.619171 \\
\hline D9J302_MOUSE & $\mathrm{ENH}$ isoform $1 \mathrm{e}$ & 2.592252 \\
\hline HDHD2_MOUSE & $\begin{array}{l}\text { Haloacid dehalogenase-like hydrolase domain-containing protein } \\
2\end{array}$ & 2.579931 \\
\hline Q3UCD9_MOUSE & Cathepsin D & 2.520039 \\
\hline Q5SW88_MOUSE & Protein Rab1a & 2.514497 \\
\hline Z4YK42_MOUSE & NAD kinase 2, mitochondrial & 2.498911 \\
\hline
\end{tabular}




\begin{tabular}{|c|c|c|}
\hline AOAOH2UH17_MOUSE & Ubiquitin associated protein 2-like, isoform CRA_b & 2.497335 \\
\hline B2RTM0_MOUSE & Histone $\mathrm{H} 4$ & 2.495948 \\
\hline Q5M9J2_MOUSE & Histidine triad nucleotide binding protein 2 & 2.442678 \\
\hline ATOX1_MOUSE & Copper transport protein ATOX1 & 2.436779 \\
\hline Q91XH5_MOUSE & Sepiapterin reductase & 2.39974 \\
\hline MIRO1_MOUSE & Isoform 4 of Mitochondrial Rho GTPase 1 & 2.379248 \\
\hline PP1B_MOUSE & Serine/threonine-protein phosphatase PP1-beta catalytic subunit & 2.369575 \\
\hline Q3U4U6_MOUSE & T-complex protein 1 subunit gamma & 2.3335 \\
\hline A0A0R4J093_MOUSE & UMP-CMP kinase & 2.320654 \\
\hline QCR10_MOUSE & Cytochrome b-c1 complex subunit 10 & 2.277176 \\
\hline COX7C_MOUSE & Cytochrome c oxidase subunit 7C, mitochondrial & 2.270833 \\
\hline CAND1_MOUSE & Cullin-associated NEDD8-dissociated protein 1 & 2.261506 \\
\hline Q543M7_MOUSE & Importin subunit alpha & 2.232002 \\
\hline Q8BVQ9_MOUSE & $26 S$ protease regulatory subunit 7 & 2.208435 \\
\hline STIP1_MOUSE & Stress-induced-phosphoprotein 1 & 2.197041 \\
\hline TXD17_MOUSE & Thioredoxin domain-containing protein 17 & 2.165337 \\
\hline Q9CPX4_MOUSE & Ferritin & 2.161834 \\
\hline F8WIA1_MOUSE & CAP-Gly domain-containing linker protein 1 & 2.122414 \\
\hline SDCG8_MOUSE & Serologically defined colon cancer antigen 8 homolog & 2.121559 \\
\hline Q8BFQ1_MOUSE & Prosaposin & 2.119551 \\
\hline PDIA6_MOUSE & Protein disulfide-isomerase A6 & 2.068221 \\
\hline Q499X4_MOUSE & Synaptic vesicle membrane protein VAT-1 homolog & 2.05867 \\
\hline Q561N5_MOUSE & $40 S$ ribosomal protein $\mathrm{S} 18$ & 2.053017 \\
\hline ES1_MOUSE & ES1 protein homolog, mitochondrial & 2.051459 \\
\hline Q3U6L3_MOUSE & Glutaredoxin, isoform CRA_a & 2.049559 \\
\hline PSA6_MOUSE & Proteasome subunit alpha type- 6 & 2.049024 \\
\hline NDUC2_MOUSE & NADH dehydrogenase 1 subunit $\mathrm{C} 2$ & 2.047106 \\
\hline H7BX64_MOUSE & Sarcolemmal membrane-associated protein & 2.045809 \\
\hline CO6A1_MOUSE & Collagen alpha-1(VI) chain & 2.038665 \\
\hline COMT_MOUSE & Catechol O-methyltransferase & 2.035218 \\
\hline ANXA7_MOUSE & Annexin A7 & 2.034 \\
\hline NDUS3_MOUSE & NADH dehydrogenase iron-sulfur protein 3 , mitochondrial & 2.0308 \\
\hline Q545S0_MOUSE & Sulfurtransferase & 2.007492 \\
\hline UGPA_MOUSE & UTP--glucose-1-phosphate uridylyltransferase & 2.00286 \\
\hline G5E8T9_MOUSE & Hydroxyacyl glutathione hydrolase & 1.99599 \\
\hline GTR4_MOUSE & Solute carrier family 2 , facilitated glucose transporter member 4 & 1.990184 \\
\hline PDC6I_MOUSE & Programmed cell death 6-interacting protein & 1.981432 \\
\hline ANXA5_MOUSE & Annexin A5 & 1.964818 \\
\hline MAOX_MOUSE & NADP-dependent malic enzyme & 1.933061 \\
\hline CD47_MOUSE & Leukocyte surface antigen CD47 & 1.93226 \\
\hline Q3TV21_MOUSE & Frataxin & 1.929493 \\
\hline ODB2_MOUSE & $\begin{array}{l}\text { Lipoamide acyltransferase component of branched-chain alpha- } \\
\text { keto acid dehydrogenase complex, mitochondrial }\end{array}$ & 1.928248 \\
\hline VAPA_MOUSE & Vesicle-associated membrane protein-associated protein $A$ & 1.90772 \\
\hline PA1B2_MOUSE & Platelet-activating factor acetylhydrolase IB subunit beta & 1.895529 \\
\hline
\end{tabular}




\begin{tabular}{|c|c|c|}
\hline NDUAD_MOUSE & NADH dehydrogenase 1 alpha subcomplex subunit 13 & 1.890973 \\
\hline MCAT_MOUSE & Mitochondrial carnitine/acylcarnitine carrier protein & 1.889925 \\
\hline BACH_MOUSE & Isoform C of Cytosolic acyl coenzyme A thioester hydrolase & 1.876667 \\
\hline Q5ND36_MOUSE & Alpha-2-antiplasmin & 1.863565 \\
\hline F2Z456_MOUSE & NADH-cytochrome b5 reductase & 1.861127 \\
\hline HSPB6_MOUSE & Heat shock protein beta- 6 & 1.847715 \\
\hline COF1_MOUSE & Cofilin-1 & 1.828459 \\
\hline PLIN4_MOUSE & Perilipin-4 & 1.827155 \\
\hline Q5HZK3_MOUSE & Proteasome (Prosome, macropain) 28 subunit, alpha & 1.816172 \\
\hline E9Q1W0_MOUSE & Calcium/calmodulin-dependent protein kinase type II subunit delta & 1.814084 \\
\hline SYDC_MOUSE & Aspartate--tRNA ligase, cytoplasmic & 1.807108 \\
\hline E9PUD2_MOUSE & Dynamin-1-like protein & 1.799964 \\
\hline Q3TX38_MOUSE & Voltage-dependent anion-selective channel protein 3 & 1.778391 \\
\hline B3AT_MOUSE & Band 3 anion transport protein & 1.77496 \\
\hline Q542G9_MOUSE & Annexin & 1.772496 \\
\hline Q80Y52_MOUSE & Heat shock protein 90, alpha (Cytosolic), class A member 1 & 1.764213 \\
\hline LAMP2_MOUSE & Lysosome-associated membrane glycoprotein 2 & 1.761354 \\
\hline B7ZCF1_MOUSE & $26 \mathrm{~S}$ protease regulatory subunit $6 \mathrm{~A}$ & 1.743877 \\
\hline Q497I3_MOUSE & Fatty acid binding protein 5 , epidermal & 1.740813 \\
\hline Q3TPZ5_MOUSE & Dynactin 2 & 1.713427 \\
\hline OXND1_MOUSE & Oxidoreductase NAD-binding domain-containing protein 1 & 1.710108 \\
\hline TBA8_MOUSE & Tubulin alpha-8 chain & 1.694346 \\
\hline BZW2_MOUSE & Basic leucine zipper and W2 domain-containing protein 2 & 1.690544 \\
\hline VWA8_MOUSE & von Willebrand factor A domain-containing protein 8 & 1.690259 \\
\hline NDRG2_MOUSE & Protein NDRG2 & 1.671721 \\
\hline Q544D4_MOUSE & Delta-sarcoglycan & 1.671108 \\
\hline GSTO1_MOUSE & Glutathione S-transferase omega-1 & 1.670085 \\
\hline BAG3_MOUSE & BAG family molecular chaperone regulator 3 & 1.664661 \\
\hline E9Q3X0_MOUSE & Major vault protein & 1.661372 \\
\hline PSMD6_MOUSE & $26 \mathrm{~S}$ proteasome non-ATPase regulatory subunit 6 & 1.649079 \\
\hline Q7JCZ1_MOUSE & Cytochrome c oxidase subunit 2 & 1.648254 \\
\hline NDUB2_MOUSE & $\begin{array}{l}\text { NADH dehydrogenase } 1 \text { beta subcomplex subunit } 2 \text {, } \\
\text { mitochondrial }\end{array}$ & 1.645909 \\
\hline Q8CBB6_MOUSE & Histone $\mathrm{H} 2 \mathrm{~B}$ & 1.645206 \\
\hline NDUA6_MOUSE & NADH dehydrogenase 1 alpha subcomplex subunit 6 & 1.643343 \\
\hline Q4FJU3_MOUSE & Crip2 protein & 1.642117 \\
\hline CATB_MOUSE & Cathepsin B & 1.640215 \\
\hline ETHE1_MOUSE & Persulfide dioxygenase ETHE1, mitochondrial & 1.634732 \\
\hline B0QZL1_MOUSE & Alpha-enolase & 1.633795 \\
\hline Q6GT24_MOUSE & Peroxiredoxin 6 & 1.626017 \\
\hline TIM13_MOUSE & Mitochondrial import inner membrane translocase subunit Tim13 & 1.615049 \\
\hline Q3T9Z2_MOUSE & Glyoxylate reductase/hydroxypyruvate reductase & 1.610213 \\
\hline G5E8R3_MOUSE & Pyruvate carboxylase & 1.597556 \\
\hline NDUS2_MOUSE & $\mathrm{NADH}$ dehydrogenase iron-sulfur protein 2, mitochondrial & 1.596723 \\
\hline D3Z636_MOUSE & Inorganic pyrophosphatase 2, mitochondrial & 1.59351 \\
\hline
\end{tabular}




\begin{tabular}{|l|l|l|}
\hline KAD1_MOUSE & Adenylate kinase isoenzyme 1 & 1.593473 \\
\hline ATAD3_MOUSE & ATPase family AAA domain-containing protein 3 & 1.586299 \\
\hline NDUV2_MOUSE & NADH dehydrogenase flavoprotein 2, mitochondrial & 1.569232 \\
\hline GDIR1_MOUSE & Rho GDP-dissociation inhibitor 1 & 1.563698 \\
\hline Q9MD82_MOUSE & NADH-ubiquinone oxidoreductase chain 5 & 1.562489 \\
\hline A2RTT4_MOUSE & MCG4297 & 1.558157 \\
\hline ENOB_MOUSE & Beta-enolase & 1.554284 \\
\hline Q3TS44_MOUSE & Proteasome subunit alpha type & 1.552829 \\
\hline G3X9J1_MOUSE & Sodium/calcium exchanger 1 & 1.550129 \\
\hline ARPC2_MOUSE & Actin-related protein 2/3 complex subunit 2 & 1.542713 \\
\hline GRPE1_MOUSE & GrpE protein homolog 1, mitochondrial & 1.538649 \\
\hline Q91V77_MOUSE & Protein S100 & 1.537994 \\
\hline F6WYM0_MOUSE & Septin-2 & 1.530294 \\
\hline HSPB1_MOUSE & Heat shock protein beta-1 & 1.52291 \\
\hline TLN1_MOUSE & Talin-1 & 1.52168 \\
\hline Q545X8_MOUSE & 40S ribosomal protein S4 & 1.519066 \\
\hline A0A087WS46_MOUSE & Elongation factor 1-beta & 1.514587 \\
\hline B9EHN0_MOUSE & Ubiquitin-activating enzyme E1, Chr X & 1.514322 \\
\hline A0A0G2JFH2_MOUSE & Microtubule-associated protein & 1.510462 \\
\hline Q9DCY1_MOUSE & Peptidyl-prolyl cis-trans isomerase & 1.504742 \\
\hline
\end{tabular}

\section{Table 3.6}

\begin{tabular}{|l|l|l|}
\hline \multicolumn{3}{|c|}{ Group C: Down-regulated Proteins KO-TAC vS KO-SHAM } \\
\hline Uniprot accession & Protein & Fold Change \\
\hline NDUA3_MOUSE & NADH dehydrogenase 1 alpha subcomplex subunit 3 & 7.130738 \\
\hline DHSD_MOUSE & $\begin{array}{l}\text { Succinate dehydrogenase cytochrome b small subunit, } \\
\text { mitochondrial }\end{array}$ & 5.199991 \\
\hline A1BG_MOUSE & Alpha-1B-glycoprotein & 4.615402 \\
\hline CXA1_MOUSE & Gap junction alpha-1 protein & 4.604655 \\
\hline NDUB3_MOUSE & NADH dehydrogenase 1 beta subcomplex subunit 3 & 2.99022 \\
\hline 68MP_MOUSE & 6.8 kDa mitochondrial proteolipid & 2.836409 \\
\hline Q8BTU6_MOUSE & Eukaryotic initiation factor 4A-II & 2.696546 \\
\hline G5E8J6_MOUSE & Histidine rich calcium binding protein, isoform CRA_a & 2.535813 \\
\hline Q58E35_MOUSE & $60 S$ acidic ribosomal protein P1 & 2.5039 \\
\hline RL8_MOUSE & $60 S$ ribosomal protein L8 & 2.355288 \\
\hline A1AT4_MOUSE & Alpha-1-antitrypsin 1-4 & 2.341611 \\
\hline FRIH_MOUSE & Ferritin heavy chain & 2.261752 \\
\hline ILK_MOUSE & Integrin-linked protein kinase & 2.200704 \\
\hline Q6P5I3_MOUSE & S-(hydroxymethyl)glutathione dehydrogenase & 2.184623 \\
\hline RLA2_MOUSE & $60 S$ acidic ribosomal protein P2 & 2.169577 \\
\hline NDUB4_MOUSE & NADH dehydrogenase 1 beta subcomplex subunit 4 & 2.161729 \\
\hline 2AAA_MOUSE & $\begin{array}{l}\text { Serine/threonine-protein phosphatase 2A 65 kDa regulatory } \\
\text { subunit A alpha isoform }\end{array}$ & 2.154025 \\
\hline
\end{tabular}




\begin{tabular}{|c|c|c|}
\hline Q497X4_MOUSE & Cathepsin R & 2.131074 \\
\hline Q3U367_MOUSE & 4-trimethylaminobutyraldehyde dehydrogenase & 2.036147 \\
\hline Q3UX28_MOUSE & Brain protein 44 -like & 2.023577 \\
\hline K22O_MOUSE & Keratin, type II cytoskeletal 2 oral & 1.984574 \\
\hline Q91VA7_MOUSE & Isocitrate dehydrogenase [NAD] subunit, mitochondrial & 1.982698 \\
\hline Q544X6_MOUSE & Ferrochelatase & 1.94918 \\
\hline E9QMK9_MOUSE & Protein 9030617003Rik & 1.948365 \\
\hline MTX2_MOUSE & Metaxin-2 & 1.925818 \\
\hline E9Q7L0_MOUSE & Protein Ogdhl & 1.919471 \\
\hline KV3A4_MOUSE & Ig kappa chain V-III region 50S10.1 & 1.917761 \\
\hline TRAP1_MOUSE & Heat shock protein $75 \mathrm{kDa}$, mitochondrial & 1.910707 \\
\hline RL14_MOUSE & 60S ribosomal protein L14 & 1.89362 \\
\hline APOH_MOUSE & Beta-2-glycoprotein 1 & 1.887297 \\
\hline B1AU25_MOUSE & Apoptosis-inducing factor 1 , mitochondrial & 1.839261 \\
\hline Q921R2_MOUSE & $40 \mathrm{~S}$ ribosomal protein $\mathrm{S} 13$ & 1.827666 \\
\hline ARF5_MOUSE & ADP-ribosylation factor 5 & 1.821445 \\
\hline MYL6_MOUSE & Myosin light polypeptide 6 & 1.812493 \\
\hline CES1D_MOUSE & Carboxylesterase 1D & 1.801977 \\
\hline Q497F1_MOUSE & Troponin I, cardiac 3 & 1.796747 \\
\hline ABCB8_MOUSE & ATP-binding cassette sub-family B member 8, mitochondrial & 1.787346 \\
\hline PPIF_MOUSE & Peptidyl-prolyl cis-trans isomerase $\mathrm{F}$, mitochondrial & 1.75551 \\
\hline LDHB_MOUSE & L-lactate dehydrogenase $B$ chain & 1.730964 \\
\hline DYL2_MOUSE & Dynein light chain 2, cytoplasmic & 1.729737 \\
\hline IVD_MOUSE & Isovaleryl-CoA dehydrogenase, mitochondrial & 1.698572 \\
\hline Q58EV5_MOUSE & High mobility group box 1 & 1.66142 \\
\hline ADCK3_MOUSE & Atypical kinase ADCK3, mitochondrial & 1.616159 \\
\hline Q3TGZ3_MOUSE & Isocitrate dehydrogenase [NAD] subunit, mitochondrial & 1.608976 \\
\hline CAD13_MOUSE & Cadherin-13 & 1.603548 \\
\hline Q6ZWZ6_MOUSE & $40 \mathrm{~S}$ ribosomal protein $\mathrm{S} 12$ & 1.601422 \\
\hline B2RS41_MOUSE & Aldhehyde dehydrogenase family 5 , subfamily $\mathrm{A} 1$ & 1.598923 \\
\hline RM12_MOUSE & $39 S$ ribosomal protein L12, mitochondrial & 1.596099 \\
\hline PLIN5_MOUSE & Perilipin-5 & 1.58571 \\
\hline RTN3_MOUSE & Reticulon-3 & 1.555692 \\
\hline SDHA_MOUSE & Succinate dehydrogenase flavoprotein subunit, mitochondrial & 1.534129 \\
\hline TPM1_MOUSE & Tropomyosin alpha-1 chain & 1.52837 \\
\hline
\end{tabular}

\section{Table 3.7}

\begin{tabular}{|l|l|l|}
\hline \multicolumn{3}{|c|}{ Group D: Up-regulated Proteins in KO-TAC vs WT-TAC } \\
\hline Uniprot Accession & Protein & Fold Change \\
\hline Q542X7_MOUSE & Chaperonin subunit 2 (Beta), isoform CRA_a & 4.011262 \\
\hline QCR10_MOUSE & Cytochrome b-c1 complex subunit 10 & 2.610662 \\
\hline G3X9J1_MOUSE & Sodium/calcium exchanger 1 & 2.551344 \\
\hline
\end{tabular}




\begin{tabular}{|c|c|c|}
\hline PSMD6_MOUSE & $26 \mathrm{~S}$ proteasome non-ATPase regulatory subunit 6 & 2.527684 \\
\hline NDUF4_MOUSE & NADH dehydrogenase 1 alpha subcomplex assembly factor 4 & 2.488534 \\
\hline Q91XH5_MOUSE & Sepiapterin reductase & 2.382154 \\
\hline Q9CPX4_MOUSE & Ferritin & 2.220084 \\
\hline NDUC2_MOUSE & NADH dehydrogenase 1 subunit $\mathrm{C} 2$ & 2.129968 \\
\hline GTR4_MOUSE & glucose transporter member 4 & 2.048086 \\
\hline NDUA6_MOUSE & NADH dehydrogenase 1 alpha subcomplex subunit 6 & 2.046164 \\
\hline Q5M9J2_MOUSE & Histidine triad nucleotide binding protein 2 & 2.004136 \\
\hline CD47_MOUSE & Leukocyte surface antigen CD47 & 1.993659 \\
\hline Q8BH80_MOUSE & Vesicle-associated membrane protein, associated protein $B$ and $\mathrm{C}$ & 1.94817 \\
\hline A3KGQ6_MOUSE & Actin-related protein $2 / 3$ complex subunit 5 & 1.892679 \\
\hline QCR8_MOUSE & Cytochrome b-c1 complex subunit 8 & 1.850327 \\
\hline NDUV2_MOUSE & NADH dehydrogenase flavoprotein 2, mitochondrial & 1.801866 \\
\hline NDUS3_MOUSE & NADH dehydrogenase iron-sulfur protein 3 , mitochondrial & 1.794648 \\
\hline Q80ZZ0_MOUSE & EH domain-containing protein 1 & 1.786371 \\
\hline TM14C_MOUSE & Transmembrane protein $14 \mathrm{C}$ & 1.77977 \\
\hline Q5SW88_MOUSE & Protein Rab1a & 1.767473 \\
\hline CATB_MOUSE & Cathepsin B & 1.757032 \\
\hline NDUAD_MOUSE & NADH dehydrogenase 1 alpha subcomplex subunit 13 & 1.752319 \\
\hline THIM_MOUSE & 3-ketoacyl-CoA thiolase, mitochondrial & 1.74534 \\
\hline Q7JCZ1_MOUSE & Cytochrome c oxidase subunit 2 & 1.739015 \\
\hline Q4FK74_MOUSE & ATP synthase subunit delta, mitochondrial & 1.726552 \\
\hline OXND1_MOUSE & Oxidoreductase NAD-binding domain-containing protein 1 & 1.715566 \\
\hline Z4YK42_MOUSE & NAD kinase 2, mitochondrial & 1.703483 \\
\hline Q5NCJ9_MOUSE & Cytochrome b-c1 complex subunit 9 & 1.680291 \\
\hline CY1_MOUSE & Cytochrome $\mathrm{c} 1$, heme protein, mitochondrial & 1.673262 \\
\hline B2RTM0_MOUSE & Histone $\mathrm{H} 4$ & 1.663071 \\
\hline Q792A4_MOUSE & Cox7ah & 1.659221 \\
\hline CPT2_MOUSE & Carnitine O-palmitoyltransferase 2, mitochondrial & 1.658698 \\
\hline Q8C5G6_MOUSE & Toll-interacting protein & 1.640463 \\
\hline A0A0R3P9C8_MOUSE & NADH dehydrogenase 1 alpha subcomplex subunit 9, mitochond & 1.637799 \\
\hline VWA8_MOUSE & von Willebrand factor A domain-containing protein 8 & 1.63688 \\
\hline Q4FJU3_MOUSE & Crip2 protein & 1.623828 \\
\hline TACO1_MOUSE & Translational activator of cytochrome $\mathrm{c}$ oxidase 1 & 1.604731 \\
\hline HHATL_MOUSE & Protein-cysteine N-palmitoyltransferase HHAT-like protein & 1.593732 \\
\hline HCDH_MOUSE & Hydroxyacyl-coenzyme A dehydrogenase, mitochondrial & 1.580108 \\
\hline F6TCV0_MOUSE & Serine protease HTRA2, mitochondrial & 1.580029 \\
\hline Q9MD82_MOUSE & NADH-ubiquinone oxidoreductase chain 5 & 1.575971 \\
\hline RTN2_MOUSE & Isoform 2 of Reticulon-2 & 1.57565 \\
\hline Q3UAI3_MOUSE & CD36 antigen, isoform CRA_a & 1.563316 \\
\hline NDUS7_MOUSE & NADH dehydrogenase iron-sulfur protein 7 , mitochondrial & 1.562736 \\
\hline
\end{tabular}


Table 3.8

\begin{tabular}{|c|c|c|}
\hline \multicolumn{3}{|c|}{ Group D: Down-regulated Proteins in KO-TAC vs WT-TAC } \\
\hline Uniprot Accessio & Protein & Fold Change \\
\hline TBB5_MOUSE & Tubulin beta- 5 chain & 2.981927 \\
\hline DHSD_MOUSE & Succinate dehydrogenase cytochrome b small subunit, mitochondrial & 2.481798 \\
\hline F6ZFU0_MOUSE & Elongation factor 1-delta & 2.425279 \\
\hline Q8BFQ1_MOUSE & Prosaposin & 2.378969 \\
\hline CAH2_MOUSE & Carbonic anhydrase 2 & 2.376344 \\
\hline GCAM_MOUSE & Ig gamma-2A chain $\mathrm{C}$ region, membrane-bound form & 2.374465 \\
\hline APOH_MOUSE & Beta-2-glycoprotein 1 & 2.372747 \\
\hline NDUA3_MOUSE & NADH dehydrogenase 1 alpha subcomplex subunit 3 & 2.266877 \\
\hline 68MP_MOUSE & $6.8 \mathrm{kDa}$ mitochondrial proteolipid & 2.150122 \\
\hline CAH1_MOUSE & Carbonic anhydrase 1 & 2.136464 \\
\hline RL8_MOUSE & $60 S$ ribosomal protein L8 & 2.089221 \\
\hline Q52L67_MOUSE & Gpsn2 protein & 2.083124 \\
\hline Q544X6_MOUSE & Ferrochelatase & 2.051581 \\
\hline B2RXX9_MOUSE & Myosin, heavy polypeptide 7 , cardiac muscle, beta & 2.026056 \\
\hline ARF5_MOUSE & ADP-ribosylation factor 5 & 1.889291 \\
\hline E9QLZ9_MOUSE & Protein enabled homolog & 1.845804 \\
\hline Q54519_MOUSE & Protein S100 & 1.809239 \\
\hline Q921R2_MOUSE & $40 S$ ribosomal protein $\mathrm{S} 13$ & 1.805659 \\
\hline MTX2_MOUSE & Metaxin-2 & 1.787365 \\
\hline A2AEX8_MOUSE & Four and a half LIM domains 1, isoform CRA_b & 1.778477 \\
\hline K22O_MOUSE & Keratin, type II cytoskeletal 2 oral & 1.770453 \\
\hline Q6P5I3_MOUSE & S-(hydroxymethyl)glutathione dehydrogenase & 1.725549 \\
\hline Q91VB8_MOUSE & Alpha globin 1 & 1.700586 \\
\hline E9Q616_MOUSE & Protein Ahnak & 1.698923 \\
\hline TPM1_MOUSE & Tropomyosin alpha-1 chain & 1.659611 \\
\hline B2MWM9_MOUSE & Calreticulin & 1.6296 \\
\hline 2AAA_MOUSE & $\begin{array}{l}\text { Serine/threonine-protein phosphatase } 2 \mathrm{~A} 65 \mathrm{kDa} \text { regulatory subunit } \mathrm{A} \\
\text { alpha isoform }\end{array}$ & 1.591433 \\
\hline Q497F1_MOUSE & Troponin I, cardiac 3 & 1.588601 \\
\hline PLAK_MOUSE & Junction plakoglobin & 1.587987 \\
\hline Q543J5_MOUSE & Antithrombin & 1.53439 \\
\hline CO3_MOUSE & Complement C3 & 1.530253 \\
\hline MLRV_MOUSE & Myosin regulatory light chain 2 , ventricular/cardiac muscle isoform & 1.528094 \\
\hline DYL2_MOUSE & Dynein light chain 2, cytoplasmic & 1.52776 \\
\hline TMOD1_MOUSE & Tropomodulin-1 & 1.517943 \\
\hline Q549A5_MOUSE & Clusterin & 1.516287 \\
\hline Q564F3_MOUSE & $40 \mathrm{~S}$ ribosomal protein $\mathrm{S} 3 \mathrm{a}$ & 1.494773 \\
\hline
\end{tabular}


A

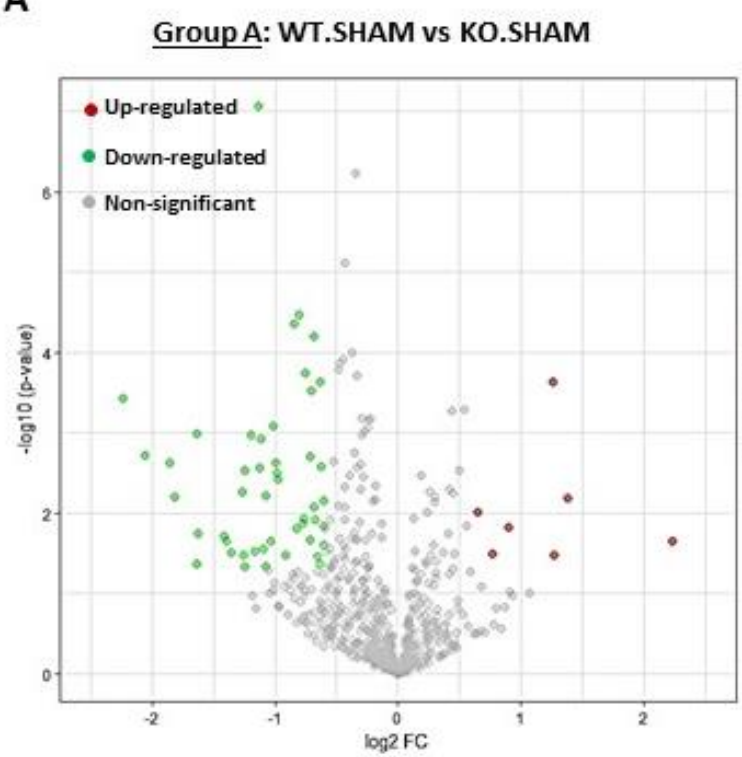

C

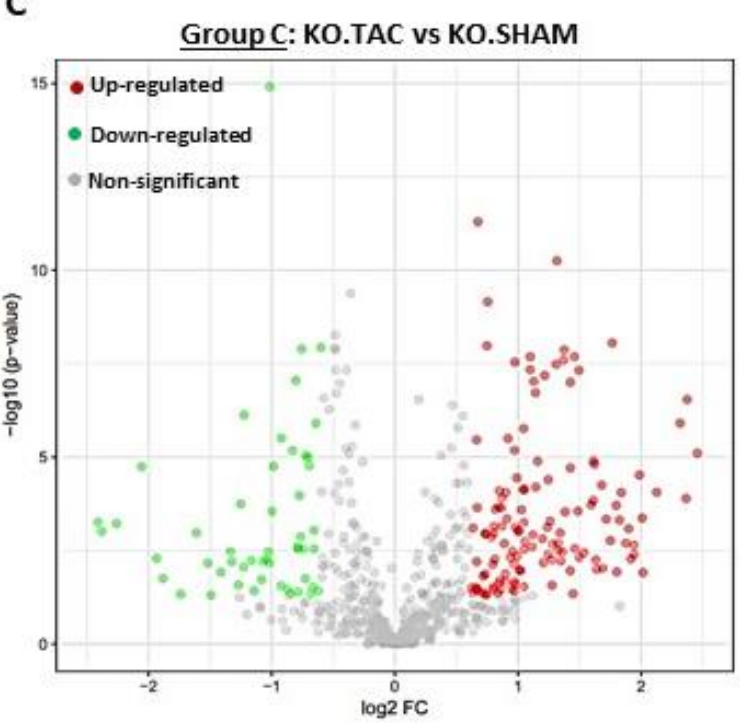

\section{B Group B: WT.TAC vs WT.SHAM}

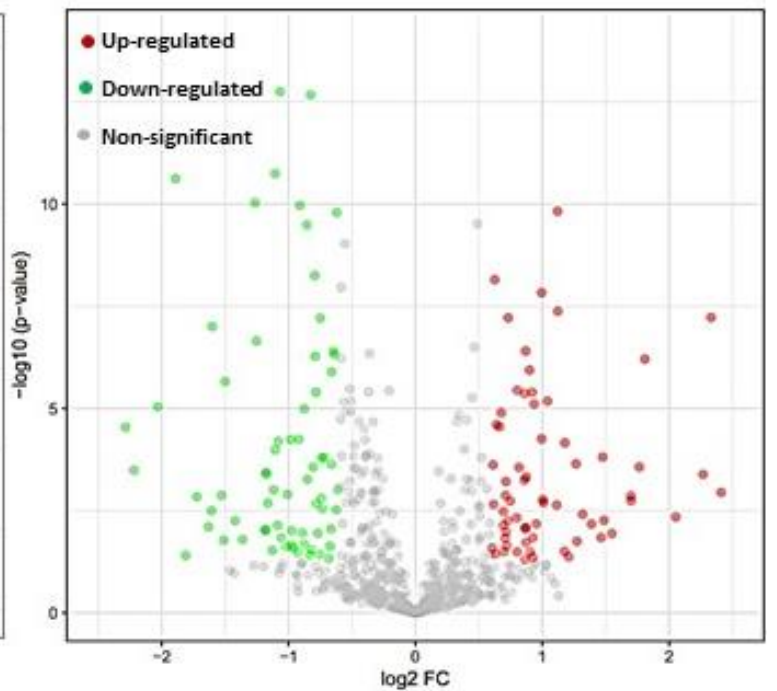

D

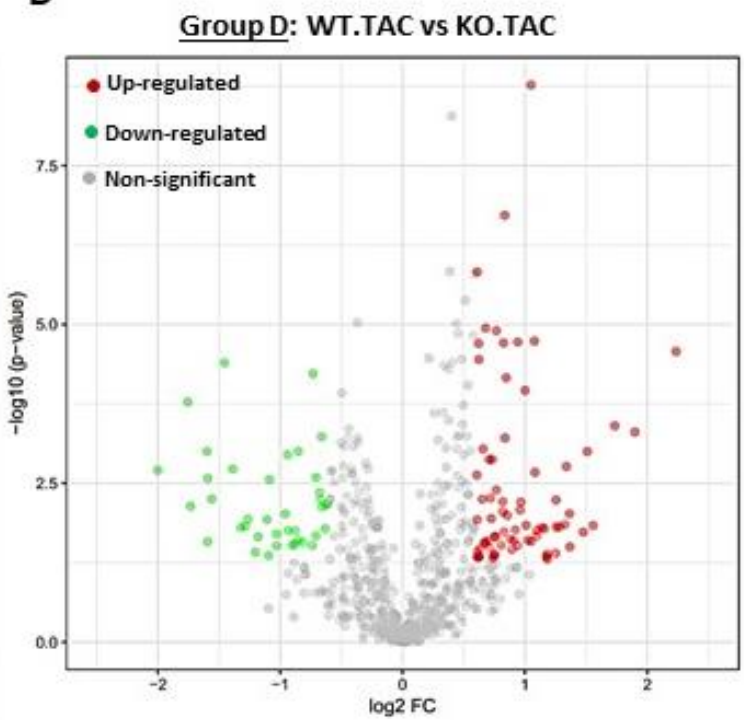

Fig. 3.17. Distribution of identified proteins in global proteome analysis: A-D Volcano plots illustrating the proteomic data log2 fold-change (log2 FC) on $X$ axis and statistical significance distribution $\log 10$ of $p$ values $(-\log 10$ ( $p$-value)) on $Y$-axis of the proteomic data. Data points in red and green indicate proteins defined to be significantly differentially expressed based upon 1.5 -fold change and $\mathrm{p}$ value of 0.005 . Proteins in red denote those that reached statistical significance using Welch's T-test and are up-regulated while Proteins in green shows significant down-regulation.

\subsubsection{Functional characterization of proteome data using STRING analysis}

For detailed characterization of proteome, we used STRING analysis to categorize differentially expressed proteins according to their subcellular localization as assigned by Gene ontology (GO) annotations for better understanding of protein functions. 


\subsubsection{Characterization of Group A (WT-SHAM vs KO-SHAM) proteome}

Previously, we observed in PCA plot (Fig. 3.15) that WT-SHAM and KO-SHAM are closely clustered to each other showing less variability. Perseus analysis showed there were only 8 up-regulated proteins in KO-SHAM as compared to WT-SHAM while there were 58 downregulated proteins. We analysed these up and down- regulated proteins using STRING analysis. We observed 5 proteins belonging to (cytosol, 63\%) while 3 proteins belong to mitochondria (37\%) in KO-SHAM (Fig. 3.18A). Up-regulated proteins are showing a small cluster of mitochondrial proteins based on their function and localisation while cytoplasmic proteins are not showing any significant link (Fig. 3.18B). Similarly, we observed out of 58 down-regulated proteins in KO-SHAM, $73 \%$ proteins belonging to cytoplasm, $17 \%$ proteins to cytoskeleton while 10\% to ribosome in (Fig. 3.18C). The STRING analysis revealed a functional link between down-regulated proteins in KO-SHAM based on their function and localisation one cluster of proteins belong to ribosomal proteins (Fig. 3.18D). Some other proteins are also linked but they do not form a major interconnecting cluster. 
A

\% Subcellular localisation of

Up-regulated Proteins

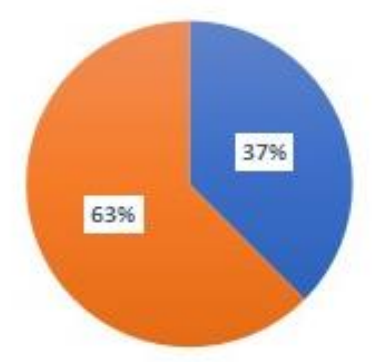

- Mitochondrial Cytoplasmic

C

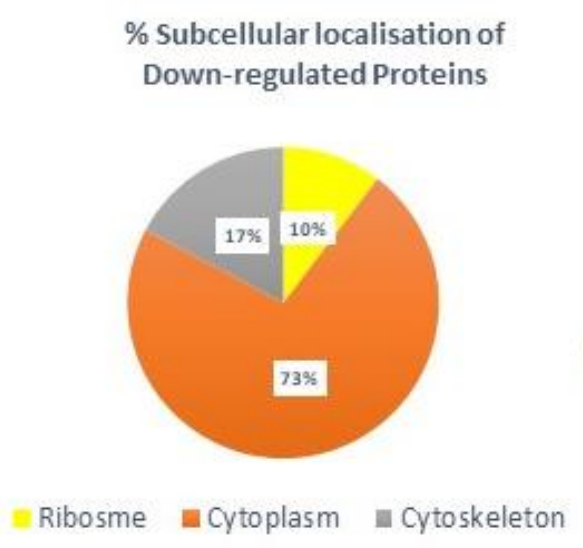

B

STRING cluster analysis

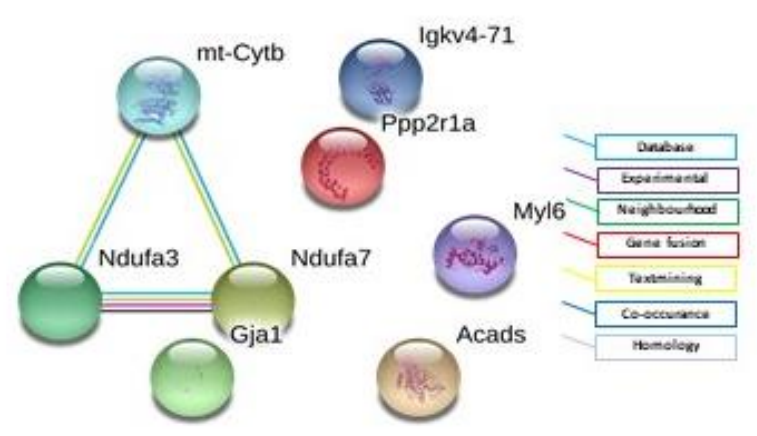

D

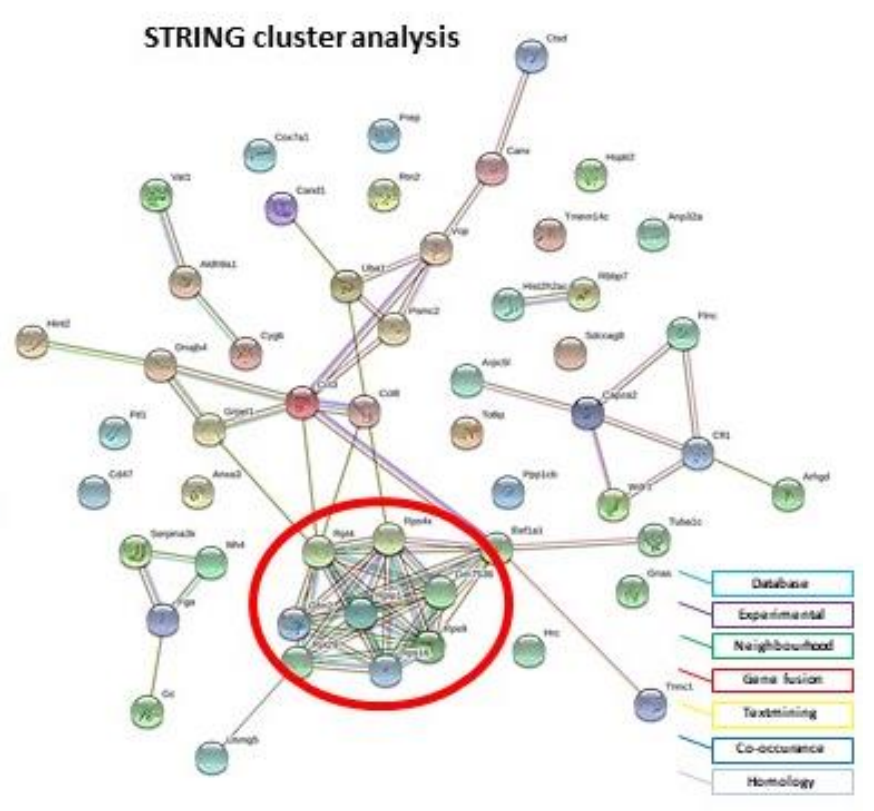

Fig. 3.18. Characterization for Group A, WT-SHAM vs KO-SHAM proteome. Pie chart shows proteins according to their subcellular location assigned by STRING and Gene-ontology (GO). Percentage of up-regulated proteins (A) and down-regulated proteins (C), Orange colour shows localised in cytoplasm, blue colour represents proteins localised in mitochondria, yellow colour represents ribosomal proteins and grey colour represents cytoskeleton proteins. Analysis of protein interaction network by STRING (B \& D), Coloured lines between the proteins indicate the protein co-occurrence, co-expression, known interactions and predicted interactions. Up-regulated proteins do not present any specific interaction or cluster (B) however Interconnecting ribosomal proteins (red circle) represents a functionally associated protein cluster in down-regulated proteins (D). B and D full view available as Supplementary Fig. S1. 


\subsubsection{Characterization of Group B (WT-SHAM vs WT-TAC) proteome}

Next, we analysed group B: WT-SHAM comparison with WT-TAC. Previously, we observed in PCA plot (Fig. 3.15) that WT-SHAM and WT -TAC are showing clusters variability which would be interesting to analyse. Perseus analysis showed there were 60 up-regulated and 70 down-regulated proteins in WT-TAC as compared to WT-SHAM.

A

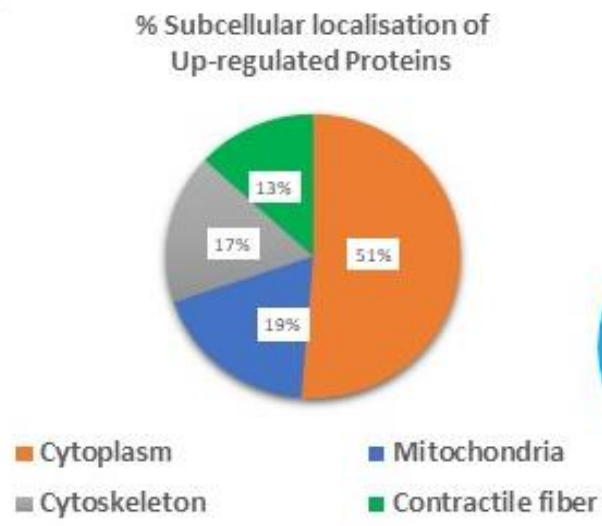

C

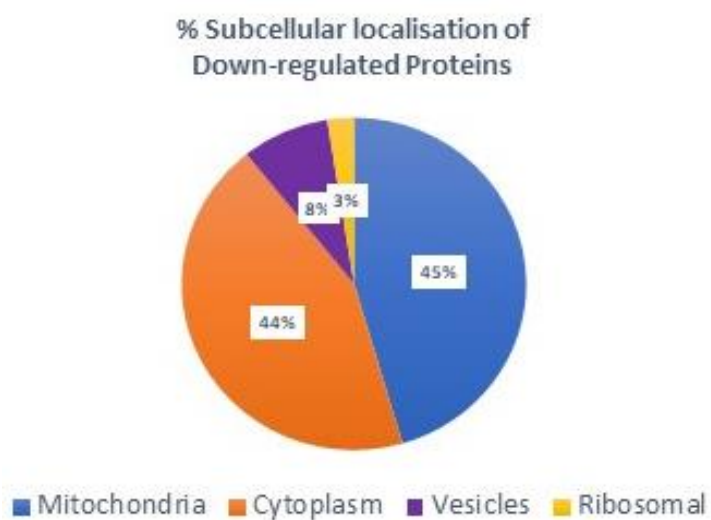

B

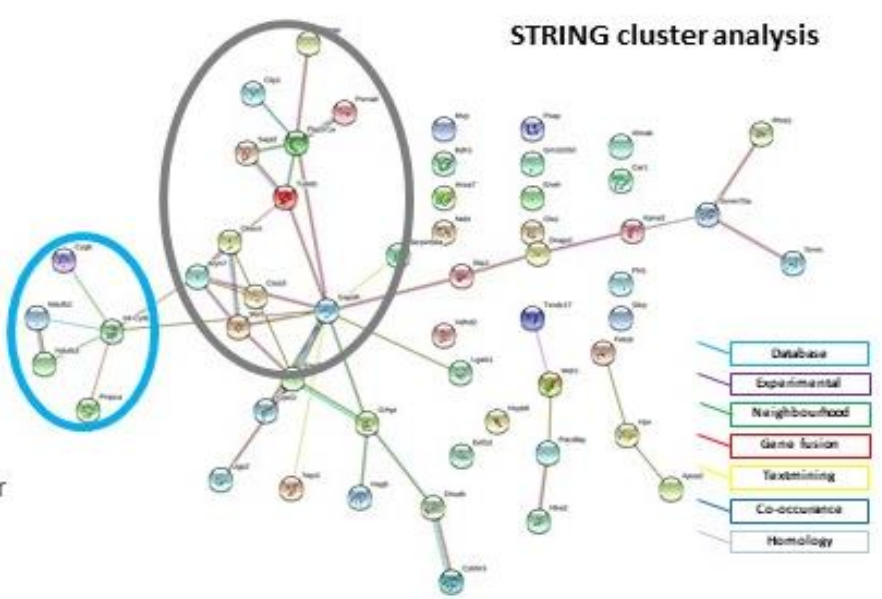

D

STRING cluster analysis

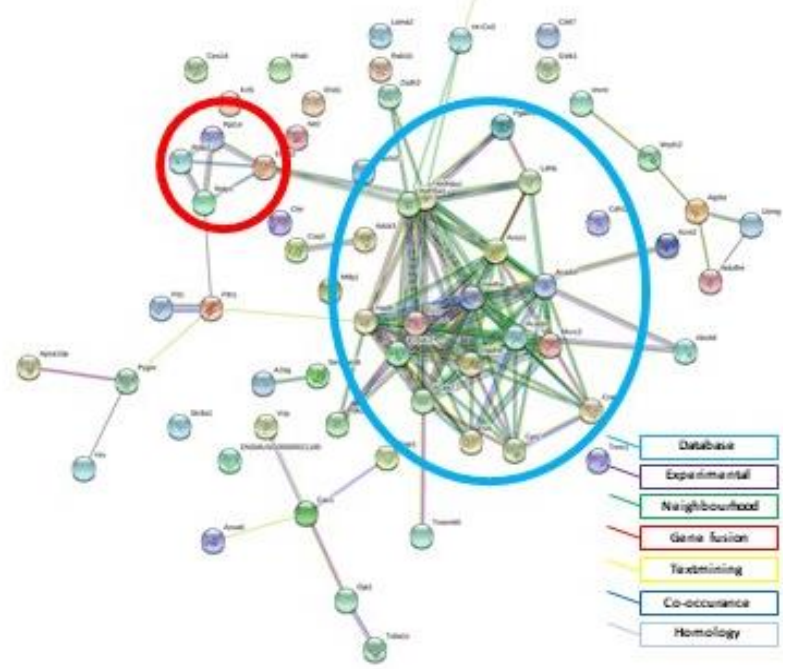

Fig. 3.19. Characterization for Group B, WT-SHAM vs WT-TAC proteome. Pie chart shows proteins according to their subcellular location assigned by STRING and Gene-ontology (GO). \%age of up-regulated proteins (A) and downregulated proteins $(\mathbf{C})$, Orange colour shows proteins localised in cytoplasm, blue colour represents the mitochondrial, green colour represents contractile fibre associated proteins, grey colour represents cytoskeleton proteins, purple colour represents vesicle associated proteins and yellow colour represents ribosomal associated proteins. Analysis of protein interaction network by STRING (B \& D), Coloured lines between the proteins indicate the protein co-occurrence, coexpression, known interactions and predicted interactions. Up-regulated proteins show interconnecting mitochondrial proteins (blue circle) represents a functionally associated protein cluster, while cytoskeleton associated proteins are showing another cluster (grey circle) in up-regulated proteins (B). Interconnecting mitochondrial proteins (blue circle) 
represents a functionally associated protein cluster, while ribosomal associated proteins are showing another cluster (red circle) in down-regulated proteins (D). B and D full view available as Supplementary Fig. S2 and S3 respectively.

We further analysed these up and down- regulated proteins using STRING analysis. We observed out of 60 up-regulated proteins in WT-TAC, $51 \%$ proteins belonging to cytoplasm, $19 \%$ to mitochondrial, $17 \%$ proteins to cytoskeleton while $13 \%$ to contractile fibre proteins (Fig. 3.19A). The STRING analysis revealed functional link between upregulated proteins in WT-TAC, based on their function and localisation. One cluster of proteins (blue circle) belong to mitochondrial proteins while second cluster (grey circle) represents cytoskeleton associated proteins (Fig. 3.19B). Some other proteins are also linked but they do not form a major interconnecting cluster. Most of them are multiple enzymes either involved in translocation of molecules or associated with different metabolic pathways. Similarly, we observed out of 70 down-regulated proteins in WT-TAC, $44 \%$ proteins belonging to cytoplasm, $45 \%$ proteins to mitochondria, $8 \%$ to vesicles while $3 \%$ to ribosome in (Fig. 3.19C). Interestingly, majority of down-regulated proteins are linked to mitochondria in WT-TAC. The STRING analysis revealed functional link between downregulated proteins in WT-TAC, based on their function and localisation. One major cluster of proteins (blue circle) belong to mitochondrial proteins while second cluster (red circle) represents Ribosomal associated proteins (Fig. 3.19D). Some other proteins are also linked but they do not form a major interconnecting cluster. Most of them are multiple enzymes which are associated with different metabolic pathways.

\subsubsection{Characterization of Group C (KO-SHAM vs KO-TAC) proteome}

Next, we analysed group C, KO-SHAM comparison with KO-TAC. Previously, we observed in PCA plot (Fig. 3.15) that KO-SHAM and KO -TAC are showing clusters variability which would be interesting to analyse. Perseus analysis showed there were 150 up-regulated and 53 down-regulated proteins in KO-TAC as compared to KO-SHAM. We further analysed these up and down-regulated proteins using STRING analysis. We observed out of 150 up-regulated proteins in KO-TAC, $40 \%$ proteins belonging to cytoplasm, $28 \%$ to vesicles, $17 \%$ to mitochondrial, $13 \%$ proteins to cytoskeleton while $2 \%$ to proteasome associated proteins (Fig. 3.20A). The STRING analysis revealed functional link between up-regulated proteins in KO-TAC, based on their function and localisation. One cluster of 
proteins (blue circle) belong to mitochondrial proteins while second cluster (green circle) represents proteasome-

A

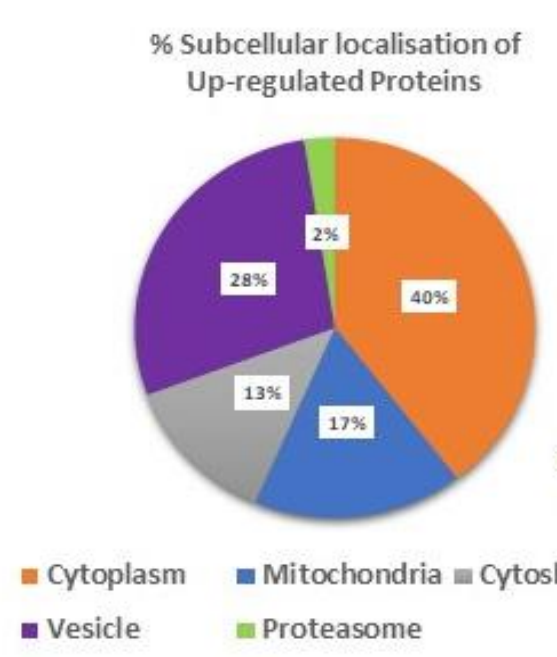

C

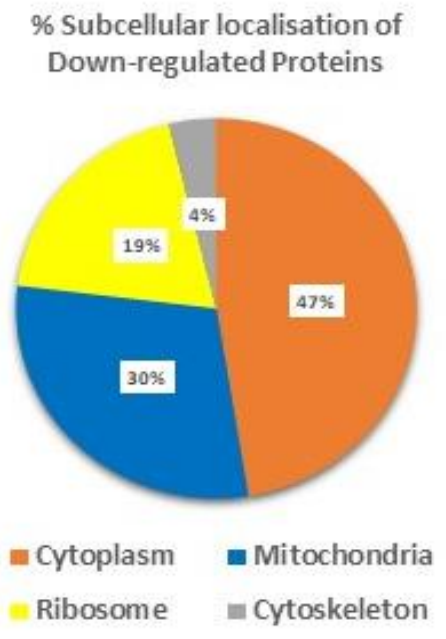

B

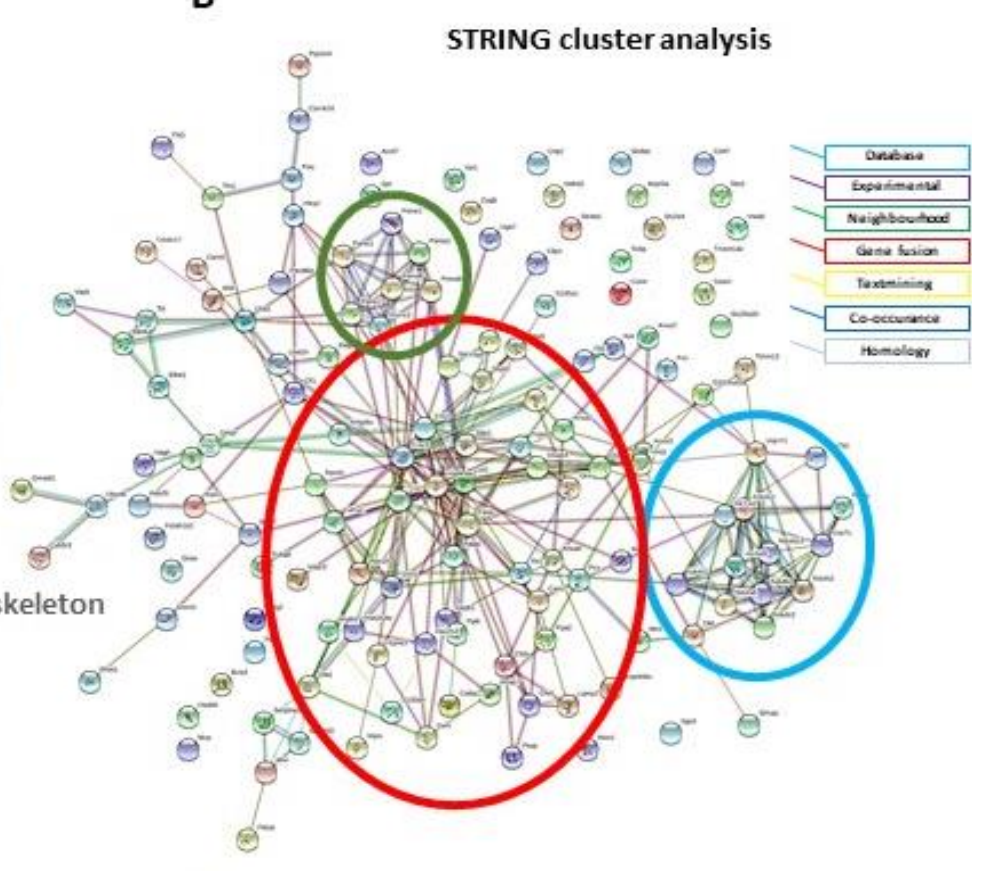

D

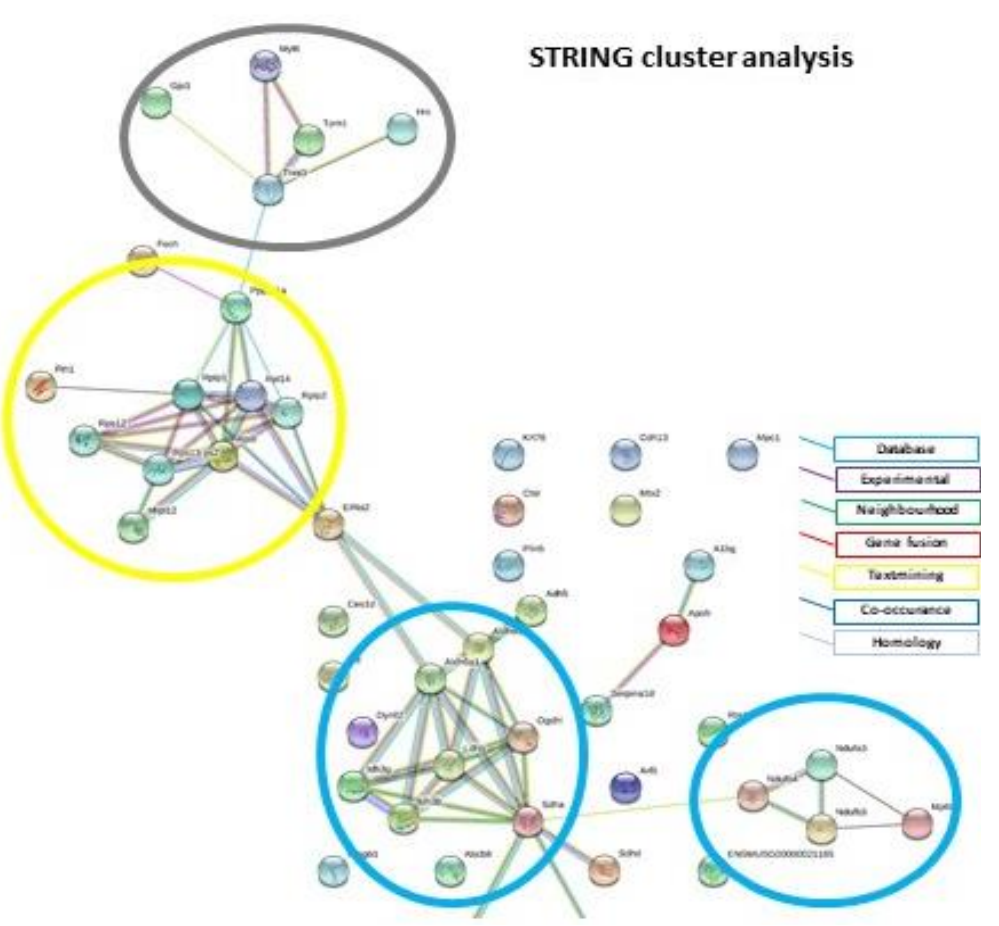

Fig. 3.20. Characterization for Group C, KO-SHAM vs KO-TAC proteome. Pie chart shows proteins according to their subcellular location assigned by STRING and Gene-ontology (GO). \% of up-regulated proteins (A) and down-regulated proteins $(\mathbf{C})$, Orange colour shows proteins localised in cytoplasm, blue colour represents the mitochondrial, green colour represents contractile fibre associated proteins, grey colour represents cytoskeleton proteins, purple colour represents vesicle associated proteins, Light-green colour represents proteasome associated proteins and yellow colour represents ribosomal associated proteins. Analysis of protein interaction network by STRING (B\& D), Coloured lines between the proteins indicate the protein co-occurrence, co-expression, known interactions and predicted interactions. Up-regulated proteins show interconnecting major cluster (red circle) represents multiple enzymes involved in metabolic pathways and cytoskeleton associated proteins while, mitochondrial proteins (blue circle) represents a functionally associated protein 
cluster, while proteasome associated proteins are showing another cluster (green circle) (B). Interconnecting major clusters show mitochondrial proteins (blue circle), ribosomal proteins (yellow circle) and cytoskeleton proteins (grey circle) in down-regulated proteins (D). B and D full view available as Supplementary Fig. S4 and S5 respectively.

-associated proteins (Fig. 3.20B), Interestingly third major cluster have proteins which are mainly enzymes either involved in translocation of molecules or associated with different metabolic pathways and some cytoskeleton associated proteins. In down-regulated proteins we observed out of 53 proteins in KO-TAC, 47\% proteins belonging to cytoplasm, $30 \%$ proteins to mitochondria, $19 \%$ to ribosome while $8 \%$ to cytoskeleton (Fig. $3.20 \mathrm{C}$ ). The STRING analysis revealed functional link between down-regulated proteins in KOTAC, based on their function and localisation. Two clusters of proteins (blue circle) belong to mitochondrial proteins, one cluster (yellow circle) associated with ribosomal proteins while one small cluster (grey circle) represents cytoskeleton associated proteins (Fig. 3.20D).

\subsubsection{Characterization of Group D (WT-TAC vs KO-TAC) proteome}

Next, we analysed group D, WT-TAC comparison with KO-TAC. Previously, we observed in PCA plot (Fig. 3.15) that WT-TAC and KO -TAC are showing clusters variability which would be interesting to analyse. Perseus analysis showed there were 43 up-regulated and 36 down-regulated proteins in KO-TAC as compared to WT-TAC. We further analysed these up and down- regulated proteins using STRING analysis. We observed out of 43 upregulated proteins in $\mathrm{KO}-\mathrm{TAC}, 47 \%$ proteins belonging to cytoplasm, 33\% to mitochondrial, while $20 \%$ to vesicles associated proteins (Fig. 3.21A). The STRING analysis revealed functional link between up-regulated proteins in KO-TAC, based on their function and localisation. One major cluster of proteins (blue circle) belong to mitochondrial proteins (Fig. 3.21B). We observed out of 36 down-regulated proteins in KO-TAC, 39\% proteins belonging to cytoplasm, $22 \%$ to vesicle associated, $21 \%$ to cytoskeleton, $10 \%$ proteins to mitochondria and $8 \%$ to ribosome (Fig. 3.21C). The STRING analysis revealed functional link between down-regulated proteins in KO-TAC, based on their function and localisation. One cluster (yellow circle) associated with ribosomal proteins while one small cluster (grey circle) represents cytoskeleton associated proteins (Fig. 3.21D). 
A

B

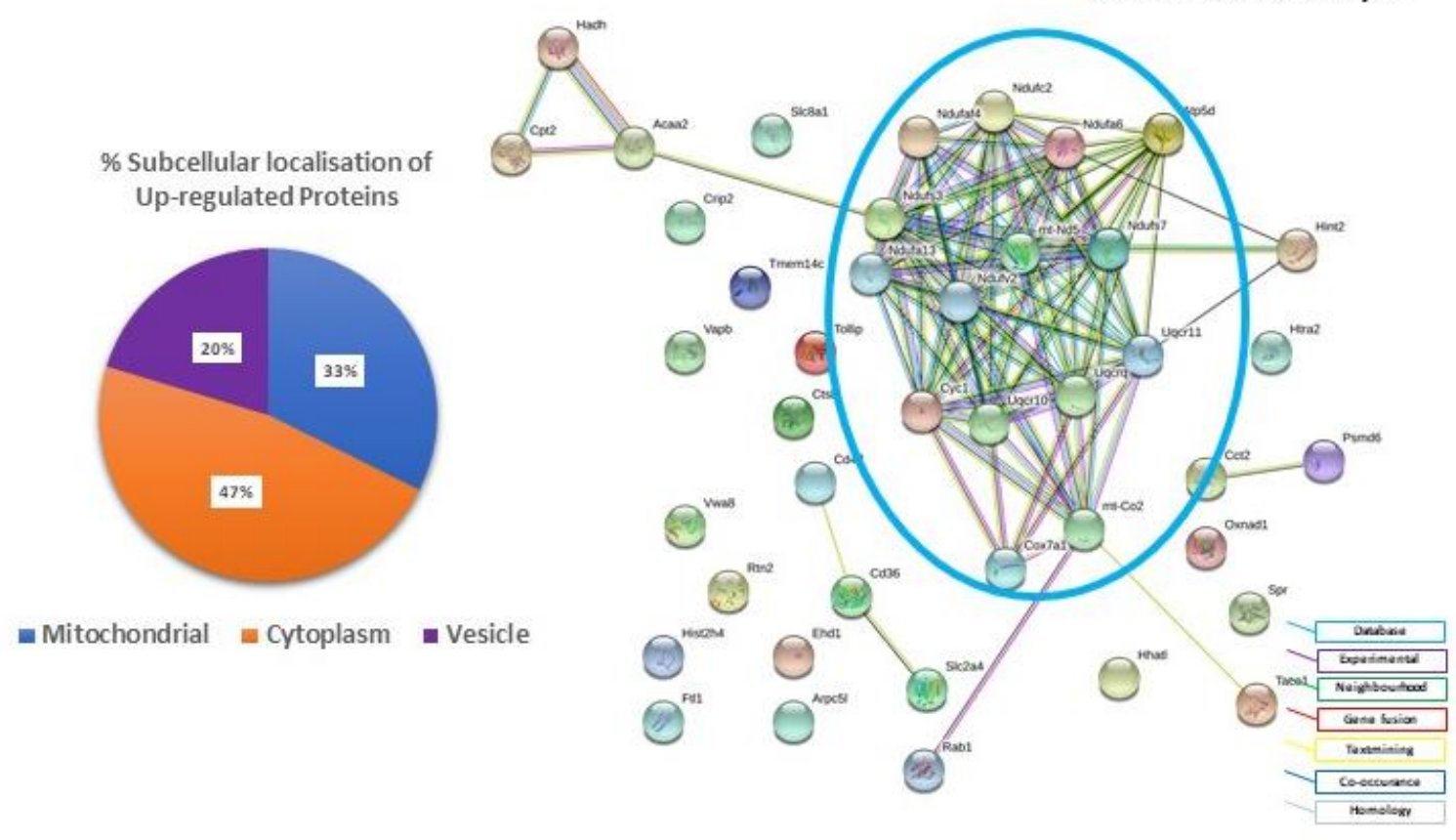

C

D

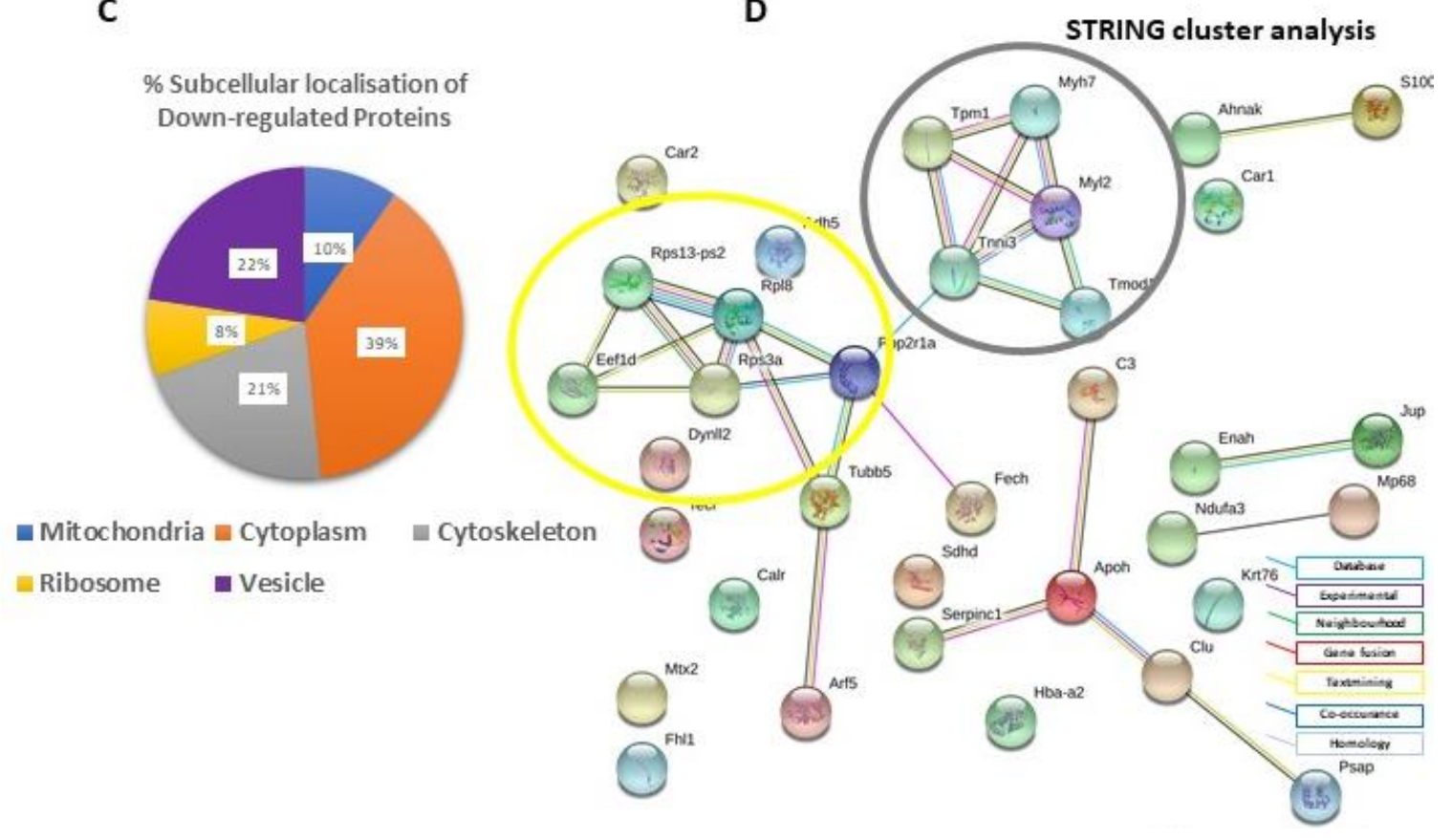

Fig. 3.21. Characterization for Group D, WT-TAC vs KO-TAC proteome. Pie chart shows proteins according to their subcellular location assigned by STRING and Gene-ontology (GO). \% of up-regulated proteins (A) and down-regulated proteins (C), Orange colour shows proteins localised in cytoplasm, blue colour represents the mitochondrial, green colour represents contractile fibre associated proteins, grey colour represents cytoskeleton proteins, purple colour represents vesicle associated proteins, and yellow colour represents ribosomal associated proteins. Analysis of protein interaction network by STRING, Coloured lines between the proteins indicate the protein co-occurrence, co-expression, known interactions and predicted interactions. Up-regulated proteins show Interconnecting major cluster shows mitochondrial proteins (blue circle) (B). Interconnecting major clusters show ribosomal proteins (yellow circle) and cytoskeleton proteins (grey circle) (D). B and D full view available as Supplementary Fig. S6 and S7 respectively. 
Interestingly in our data, group D (WT-TAC/KO-TAC) up-regulated proteins were associated with mitochondrial function in functional annotation and in cluster analysis (Fig. 3.19B). We further investigated the proteins which were associated with mitochondria and found out NADH dehydrogenase complex (Complex I of ETC) proteins were dominating the cluster (Fig. 3.19B). It is interesting to note that mitochondrial Complex I deficiency in the mouse heart results in severe hypertrophic cardiomyopathy due to selective loss of Ndufs4 (Chouchani et al., 2014). We found 9 out of 45 subunits of Complex I to be upregulated in KO-TAC mice.

\subsubsection{Ingenuity Pathway Analysis (IPA) for significantly differentiated proteins in Group C and D}

Then we concentrated more on KO-TAC vs KO-SHAM (Group C) and KO-TAC vs. WTTAC (Group D) groups to identify signalling pathways that are differentially regulated in KO-TAC hearts and might have been responsible for observed cardiac hypertrophy rescue.

For this reason, we employed IPA tool, which confirmed that the identified proteins of KOTAC mice hearts are majorly associated with mitochondrial dysfunction ( $p$-value $9.72 e^{-13}$ ), Oxidative phosphorylation ( $p$-value $6.44 \mathrm{e}^{-13}$ ), and Sirtuin signalling pathway ( $p$-value $1.47 \mathrm{e}^{-}$ ${ }^{06}$ ) (Fig. 3.22A-B). Both STRING and IPA analyses highlighted increased number of proteins belongings to mitochondrial activity and oxidative phosphorylation in KO-TAC mice hearts. These analyses suggest that restored mitochondrial function might be a contributing factor in improved cardiac function. In order to verify this hypothesis, we made use of our in-vitro model of inflammation and investigated the effect of PTP1b inhibition on mitochondrial activity. 
A

\begin{tabular}{|c|c|c|}
\hline \multicolumn{3}{|l|}{ Top Canonical Pathways } \\
\hline Name & $p$-value & Overlap \\
\hline Oxidative Phosphorylation & $6.44 \mathrm{E}-13$ & $8.3 \% 9 / 109$ \\
\hline Mitochondrial Dysfunction & $9.72 \mathrm{E}-13$ & $5.8 \% \quad 10 / 171$ \\
\hline Sirtuin Signaling Pathway & $1.47 \mathrm{E}-06$ & $2.4 \% 7 / 292$ \\
\hline TCA Cycle II (Eukaryotic) & $1.38 \mathrm{E}-05$ & $12.5 \% 3 / 24$ \\
\hline Acute Phase Response Sig & $3.39 \mathrm{E}-04$ & $2.4 \% \quad 4 / 170$ \\
\hline
\end{tabular}

B

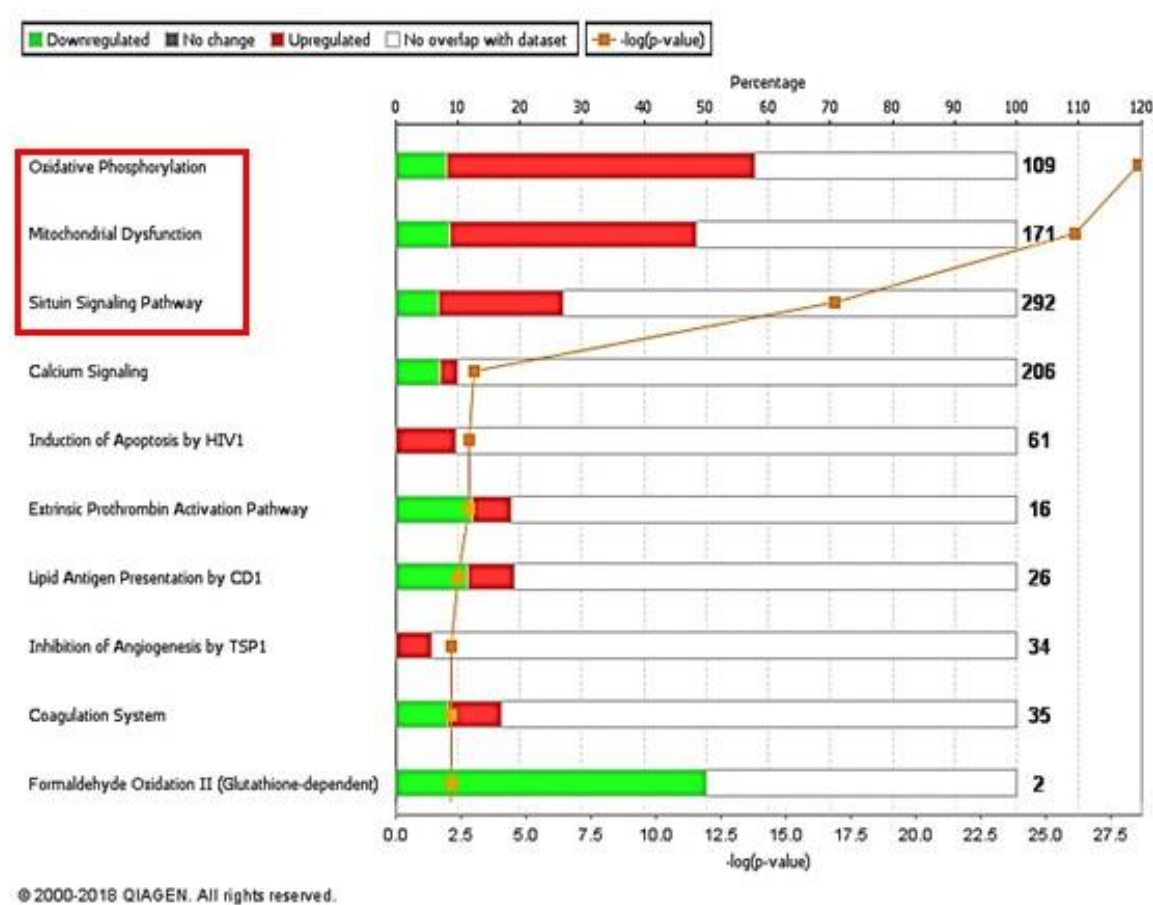

Fig. 3.22. Ingenuity pathway analysis (IPA) of KO-TAC vs WT-TAC proteins: A. Top Canonical Pathways displays the most significant Pathways across the entire dataset. The significance values of the canonical pathways were calculated by Fisher's exact test right-tailed. B. Stacked Bar chart plotted between -log of p-values (orange) and percentage are displaying the number of up-regulated (red), down-regulated (green) proteins, and orange square represents the -log of $p$-values of proteins from data set, in each Canonical Pathway.

\subsubsection{PTP1b Inhibition in HUVECs}

To inhibit the function of PTP1B in HUVECs we decided to use Claramine Sulphate, a known and commercially available inhibitor of PTP1B. To establish the concentration of Claramine required for efficient inhibition of PTP1B function, we treated HUVECs with different concentrations of Claramine (10,50 and $100 \mu \mathrm{M})$ in four groups 1. Control - non-

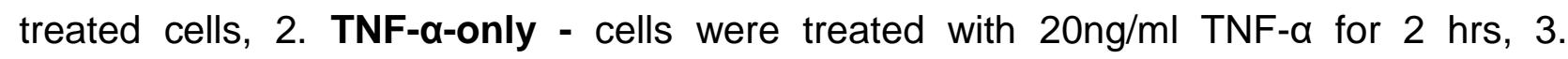
Claramine-only - cells were treated with either 10,50 or $100 \mu \mathrm{M}$ Claramine for $30 \mathrm{~min}$ and 
4. Claramine+TNF- $\alpha$ - cells were treated with either 10,50 or $100 \mu \mathrm{M}$ Claramine for 30 mins followed by TNF- $\alpha$ treatment for 2 hrs. Cells were harvested after treatment and analysed for the expression levels of $p$-AKT, which is a target of PTP1B to confirm the inhibition of PTP1B function. TNF- $\alpha$ treated cells showed reduced expression of $p-A K T$, while $100 \mu \mathrm{M}$ of Claramine-only showed significantly higher expression of p-AKT as compared to control cells (Fig. 3.23). When cells are treated with Claramine followed by TNF- $\alpha$, there is no difference in expression of $\mathrm{p}-\mathrm{AKT}$ as compared to non-treated control. This data indicates that PTP1B inhibition leads to increased phosphorylation in of AKT, however, TNF- $\alpha$ treatment reverses this phosphorylation to basal level. After successfully establishing the PTP1B inhibition model in HUVECs, we went on to study the role of PTP1B in restoring mitochondrial function.
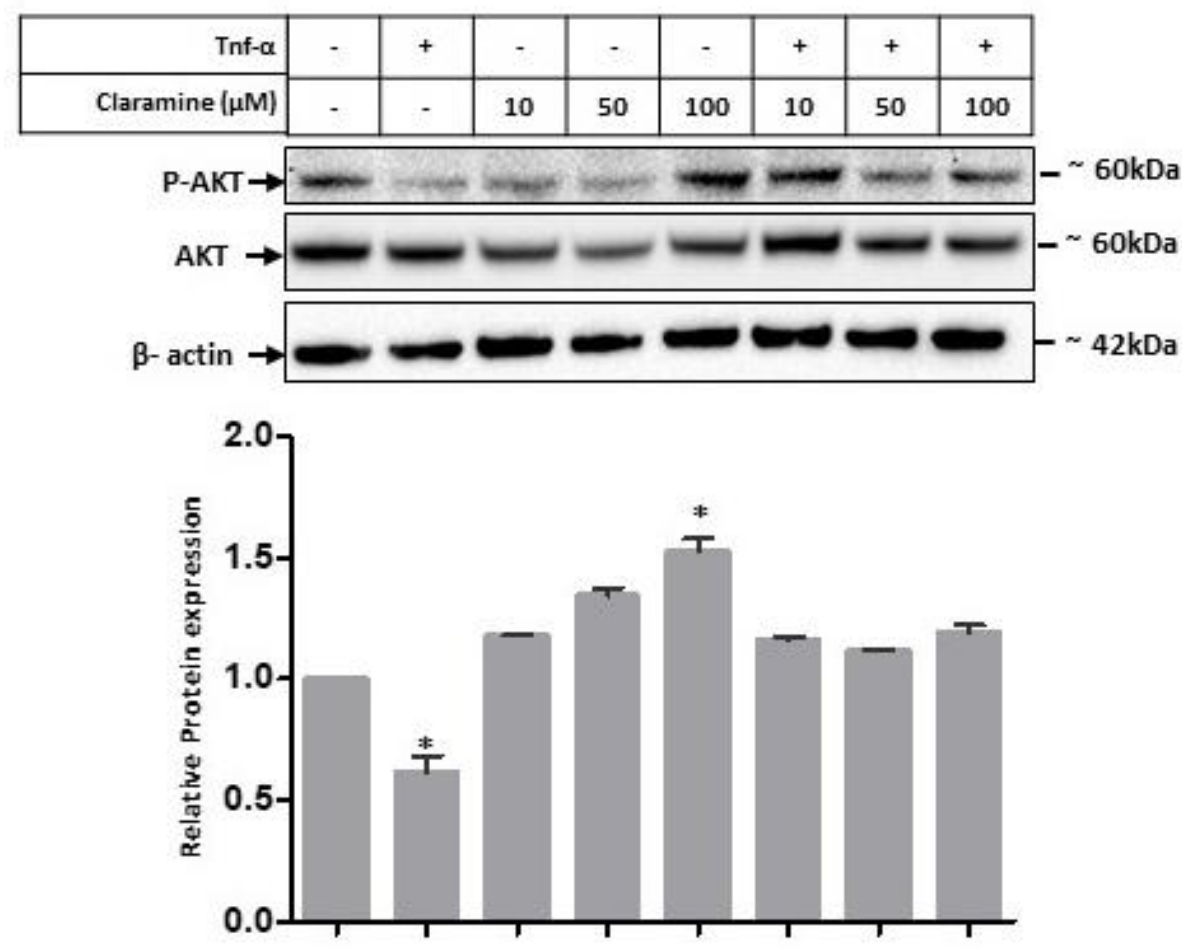

Fig. 3.23. PTP1B inhibition in HUVECs. Western Blot shows relative Protein expression of $p$-AKT normalised to housekeeping $\beta$-actin then to AKT in Control, TNF- $\alpha$-only, Claramine-only $(10,50,100 \mu \mathrm{M})$, Claramine $(10,50,100 \mu \mathrm{M})+\mathrm{TNF}-$ $\alpha$. Values are mean \pm SEM of two biological replicates and asterisk indicate statistical significance of ${ }^{*} p<0.05$ compared to control group. 


\subsubsection{Mitochondrial function assay}

To study the role of PTP1B in mitochondrial function, we treated HUVECs in five different groups 1. Control non-treated cells, 2. Claramine-only - cells treated with Claramine for $30 \mathrm{~min}, 3$ : TNF- $\alpha-o n l y$ - cells treated with $1500 \mathrm{units} / \mathrm{ml}$ of TNF- $\alpha$ for $6 \mathrm{hrs}$ to induce mitochondrial dysfunction, 4: Claramine+TNF- $\alpha$ - in which cells were pre-treated with Claramine for $30 \mathrm{~min}$ followed by TNF- $\alpha$ for $6 \mathrm{hrs}$ and 5: Mitochondrial toxin - cells treated with Sodium Azide $100 \mu \mathrm{M}$ as a positive control of mitochondrial toxicity. Thereafter, Cytotoxicity and ATP production was measured by using Mitochondrial ToxGlo ${ }^{\text {TM }}$ Assay kit (Promega).

The Mitochondrial ToxGlo ${ }^{\mathrm{TM}}$ Assay is a live cell-based assay that measures mitochondrial dysfunction. The assay detects the changes in cell membrane integrity and cellular ATP levels. Firstly, cell membrane integrity is analysed via detection of dead cell protease activity. If treatments are cytotoxic then the added fluorogenic peptide can cross the cell membrane and give significant signal as compared to viable cells of control. Next, ATP detection reagent is used to lyse the cells and generate a luminescent signal. To minimise the ATP production from glycolysis, all treatments were done in DMEM containing Galactose and in the absence of serum to avoid Glucose. Our data showed that there is a reduced ATP production when cells were treated with TNF- $\alpha$ or Positive controlmitochondrial toxin, highlighting the mitochondrial dysfunction during inflammatory response. However, pre-treatment with PTP1B inhibitor, Claramine, protects HUVECs from TNF- $\alpha$ induced mitochondrial dysfunction (Fig. 3.24). In parallel, cytotoxicity was measured, which indicated no significant difference among various treatments, indicating that the observed depletion of ATP, especially in TNF- $\alpha$ treatment, is not due to necrosis, but rather due to the inherent mitochondrial dysfunction. 


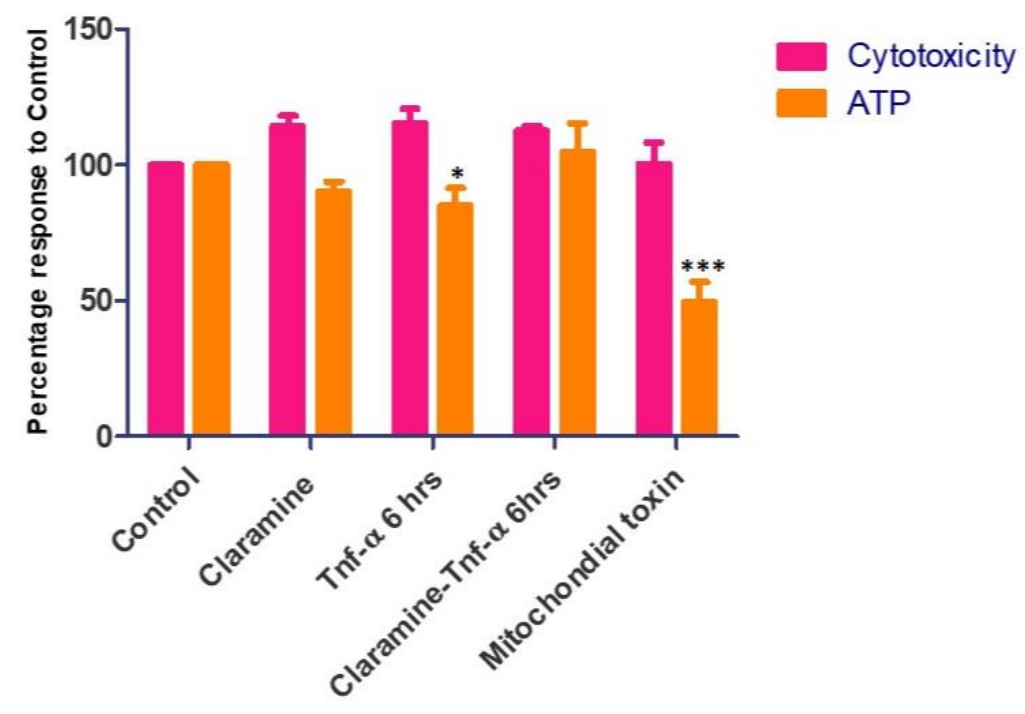

Fig. 3.24. Evaluation of mitochondrial toxicity in in-vitro inflammatory model. HUVECs were treated in following groups as 1. not-treated control, 2. Claramine only-, TNF- $\alpha-o n l y-$, Claramine+TNF- $\alpha$ and Mitochondrial toxin (Sodium Azide). The percentage of Cytotoxicity (pink) and ATP production (orange) was measured by using Mitochondrial ToxGlo kit (Promega). Values are mean \pm SEM of two biological replicates and asterisks indicate statistical significance of ${ }^{* * *} p<$ 0.001 , and ${ }^{*} p<0.05$.

Our data showed that KO-TAC heart improved cardiac function after deletion of endothelial specific PTP1B because of improved mitochondrial function. 


\section{Discussion}

Atherosclerosis, a chronic inflammatory disorder of vascular system, is one of the major contributing cause in development and progression of CVDs including coronary artery disease and heart attack (Verstraete, 1990; Zampetaki et al., 2010). Inflammatory response can lead to ECs activation and dysfunction which is considered as initiative trigger in the development of atherosclerosis (Chien, 2008; Xiao et al., 2014). Hypertension, hyperglycemia, disturbed blood flow, hypercholesteremia, and inflammatory mediators in blood like LPS (secreted by microbes) and TNF- $\alpha$ (secreted by cells, in response to LPS) can initiate inflammatory response in ECs via activation of NF-KB (Fig. 4.1) (Bleakley et al., 2015; Dart and Chin-Dusting, 1999; Hadi et al., 2005; Higashi et al., 2012; Messner and Bernhard, 2014).

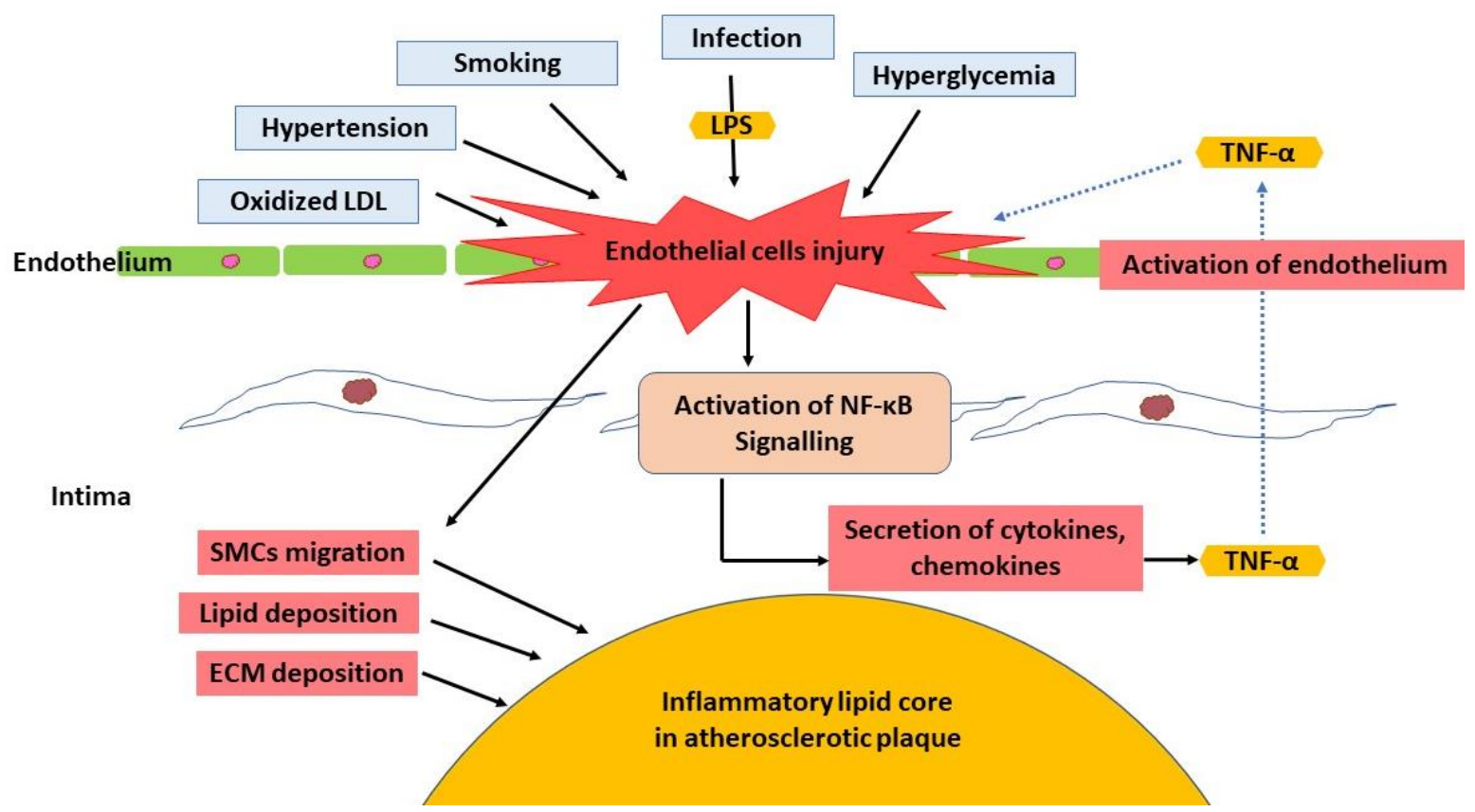

Figure 4.1. Factors involved in ECs activation, inflammation, and in atherosclerotic plaque development Factors involved in ECs activation, inflammation, monolayer disruption and progression of atherosclerotic plaque development. Smoking, hypertension, hyperglycemia, and LPS secreted by bacteria can cause ECs injury which results in activation of NF-KB signalling pathway. Cytokines, chemokines and growth factor secreted by activated ECs participate in further the development of atherosclerotic plaque.

Among these causative agents LPS and TNF- $\alpha$, pro-inflammatory mediators, are used for establishing in-vitro inflammatory models. LPS and TNF- $\alpha$ lead to ECs activation, expression of adhesion molecules on ECs and activation of various signalling pathways including NF-KB pathway. LPS secreted by bacteria activates toll like receptor 4 (TLR4), 
which leads to CD14 dependent activation of Src-kinase which phosphorylates Caveolin1 (Cav-1) (Wong et al., 2004). Phosphorylated Cav-1 leads to formation of a complex including Interleukin receptor associated kinase 1 (IRAK1), (IRAK4) and Myeloid differentiation primary response 88 (MyD88) which leads to NF-KB signalling pathway activation (Fig. 4.2) (Jiao et al., 2013; Wong et al., 2004).

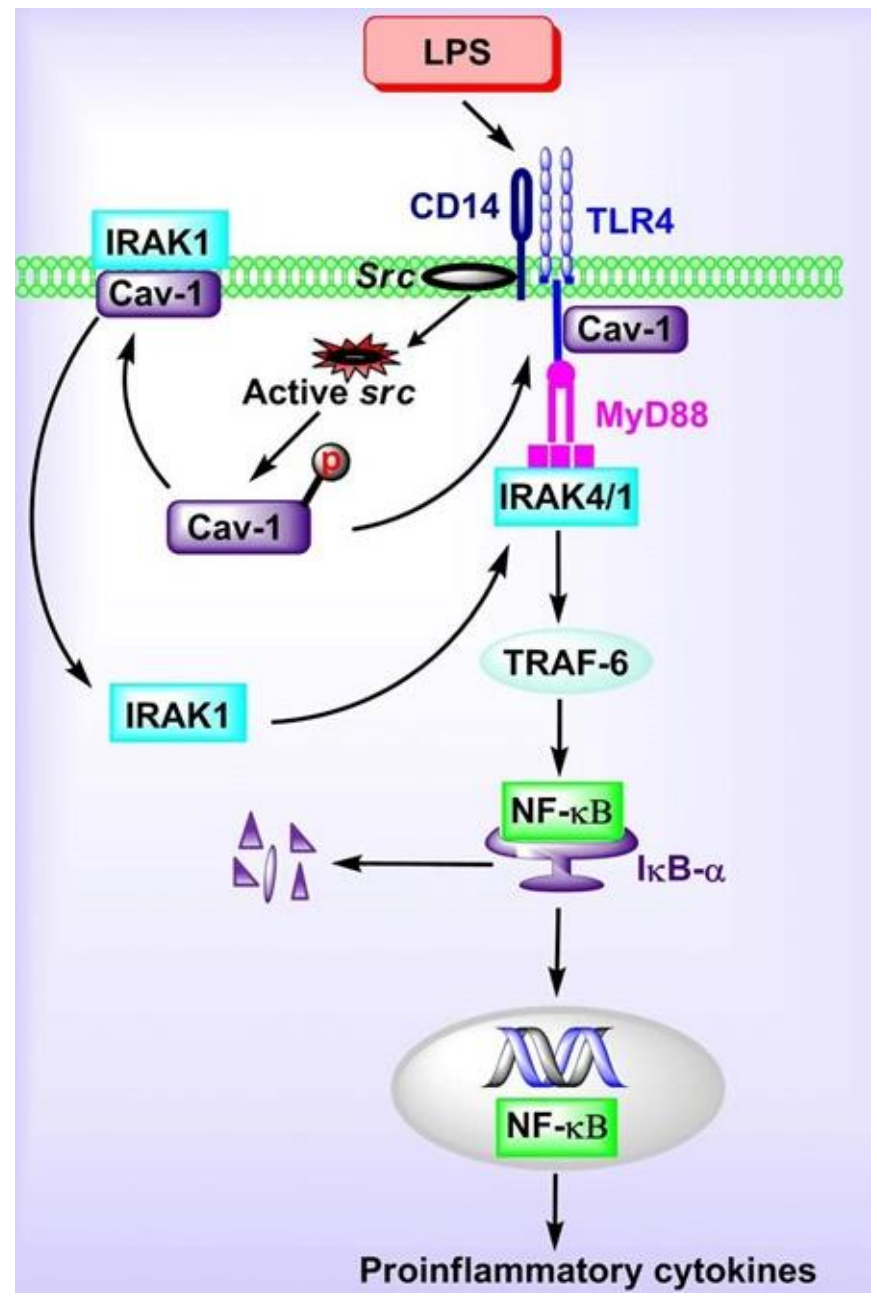

Figure 4.2. LPS mediated NF-KB activation LPS activates TLR4 which leads to CD14 mediated activation of Srckinase which phosphorylates Cav-1. Phosphorylated Cav-1 leads to formation of IRAK1-IRAK4-MyD88 complex formation which leads to TRAF-6 based activation of IKB kinase which activates NF-KB signalling pathway (Figure published by Jiao et al., 2013).

TNF- $\alpha$, a proinflammatory cytokine, binds to ECs surface via tumor necrosis factor receptor (TNFR) and activates NF-KB signalling pathway via activation of IKB kinase. TNF- $\alpha$ stimulation leads to multiple changes leading to ECs dysfunction (Zhang et al., 2009). These changes include reduced eNO production, reduced ECs specific vasodilation and vascular repair. It also increases oxidative stress, apoptosis, vascular inflammation, cell 
infiltration which contributes in the development of atherosclerosis (Fig. 4.3) (Zhang et al., 2009).

$\mathrm{Nf}-\mathrm{kB}$, a key mediator of inflammation, translocates from cytoplasm into nucleus and initiates transcription of pro-inflammatory markers (Brown et al., 2014; Xiao et al., 2014). Inflammatory markers include adhesion molecules like VCAM-I and SELE which are expressed on activated ECs and binds to monocytes (Gerhardt and Ley, 2015). Activated ECs also release cytokines like IL6 and IL8 which further recruits immune cells to ECs (Gerhardt and Ley, 2015; Ramji and Davies, 2015).

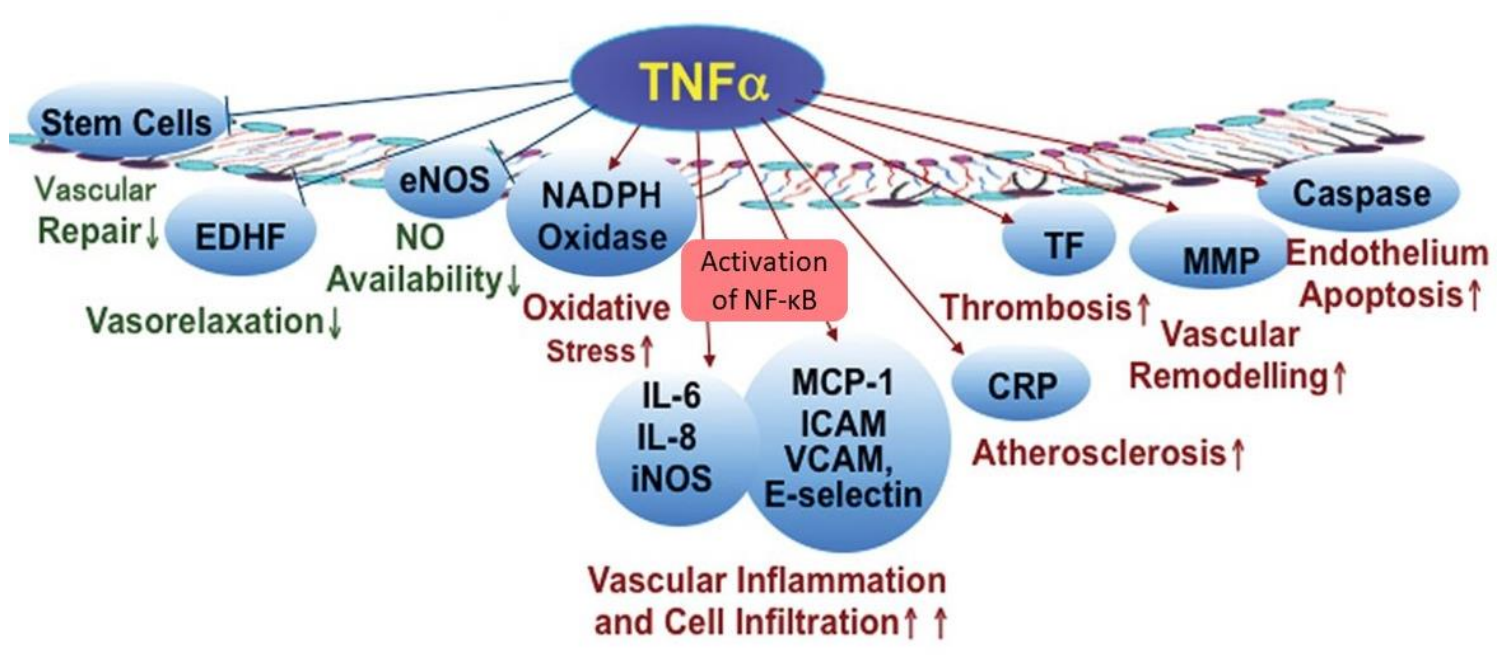

Figure 4.3. TNF- $\alpha$ mediated NF-kB activation contributes in ECs dysfunction TNF- $\alpha$ activates TNFR which leads to activation of IKB kinase which activates NF-KB signalling pathway. Activated NF-KB contributes in inflammation and increases cellular infiltration across ECs monolayer (Figure adapted from Zhang et al., 2009).

We used HUVECs as our in-vitro model for induction of inflammation in atherosclerosis and first to study the function of BRD4 in this model system. In agreement with the literature, HUVECs treated with TNF- $\alpha$ showed successful translocation of NF-KB into nucleus as compared to control cells. Our data shows significant increase of proinflammatory markers SELE and VCAMI in HUVECs after TNF- $\alpha$ treatment. Together, localisation of NF-KB inside the nucleus and overexpression of pro-inflammatory markers confirms the establishment of inflammatory phenotype in HUVECs.

BRD4 is an epigenetic factor and a member of transcription initiation machinery. It recognises and binds to the acetylated chromatin and recruit RNA polymerase to initiate transcription (Crowe et al., 2016; Devaiah et al., 2016; Devaiah et al., 2012). BRD4 is involved in multiple cellular activities but its role in inflammation came into view in 2013 
where BRD4 was reported as a partner of NF-KB on chromatin during inflammation (Xu and Vakoc, 2014a). To elaborate the role of BRD4 during inflammation, we used HUVECs as in-vitro model. We first used JQ1, a classical inhibitor of BRD4 to inhibit the function of BRD4. JQ1 binds to bromodomain I and II of BRD4 with hydrogen bonding interaction and blocks its ability to bind to acetylated chromatin (Bid and Kerk, 2016). We first treated the HUVECs with JQ1 followed by TNF- $\alpha$ treatment. Our data showed that pre-treatment with $500 \mathrm{nM}$ of JQ1 for 4 hrs significantly reduced the expression of inflammatory markers. We observed 10-fold and 17-fold reduction in expression of SELE and VCAM-I, respectively, as compared to TNF- $\alpha$-only treated cells. These results are in support of previous studies that showed BRD4 binds to SE regions of pro-inflammatory genes along with NF-KB during inflammation. These results also indicate that the inhibition of BRD4 function reduces the expression of $B R D 4$ and pro-inflammatory markers during inflammation.

Further on our immunostaining data showed that inhibition of BRD4 function did not affect the subcellular localization of $\mathrm{Nf}-\mathrm{KB}$. These results confirm that JQ1 treatment does not affect the NF-KB translocation into the nucleus during inflammatory response and the observed reduced expression of inflammatory markers is due to inhibition of BRD4 function.

JQ1 is an efficient inhibitor of BRD4, however, it also interacts with other members of BET family (Huang et al., 2016; Huang et al., 2017). Short half-life of JQ1 also make it unsuitable for animal studies and clinical trials (Wadhwa and Nicolaides, 2016). Hence, we decided to analyse another inhibitor of BRD4, RVX208, which is orally active and was already in clinical trials for atherosclerosis, diabetes, Alzheimer's disease, and chronic kidney disease (Picaud et al., 2013; Wadhwa and Nicolaides, 2016). It has been reported that RVX208 treatment increases the apolipoprotein A-I (Apo-A-I) and High-density cholesterol $(\mathrm{HDL})$ in blood which reduces multiple adverse effects observed in cardiac patients (Ghosh et al., 2017). Interestingly, BRD4, but not BRD2 and BRD3, knockdown increases the mRNA levels of Apo-A-I confirming it as a target of BRD4 (Zengerle et al., 2015). Isothermal titration calorimetry studies on BET proteins with RVX208 showed 4-fold higher binding affinity of RVX208 to BRD4 as compared to other BET family members (Kempen et al., 2013).

Collectively from literature review we know that RVX208 is a stable, orally active, already in clinical trials drug which blocks BRD4 activity and improves the cardiac function (Gilham 
et al., 2016). Keeping these facts in mind, we decided to comparatively analyse effects of RVX208 and JQ1 in our studies. Previous studies have used variable concentrations of JQ1 for variable time points including $250 \mathrm{nM}$ for 6 hrs (Huang et al., 2016), $500 \mathrm{nM}$ for 3 hrs (Huang et al., 2017), $500 \mathrm{nM}$ for 4 hrs (Picaud et al., 2013), $500 \mathrm{nM}$ for $24 \mathrm{hrs}$ (Bid et al., 2016) and 1-2 $\mu \mathrm{M}$ of JQ1 for 24 hrs (Bid et al., 2016).

As per our knowledge there are no studies reporting the RVX208 treatment on HUVECs. However, we found two studies on primary human hepatocytes which showed treatment with $30 \mu \mathrm{M}$ RVX208 for 24 and 48 hrs for inhibition of BRD4 (Gilham et al., 2016; Wasiak et al., 2016). Another study on C11 macrophage cell line used 50 to $100 \mu \mathrm{M}$ of RVX208 for 72 hrs (Lu et al., 2017). In order to establish RVX208 concentration necessary to inhibit the inflammatory phenotype, we initially used different concentrations of RVX208 for 4 hrs which did not reduce the expression of inflammatory markers even at higher concentration of $200 \mu \mathrm{M}$. We treated HUVECs with 30 and $60 \mu \mathrm{M}$ of RVX208 for 12-, 24- and 48-hrs followed by TNF- $\alpha$ for $12 \mathrm{hrs}$. We observed $12 \mathrm{hrs}$ pre-treatment was significantly reducing the expression of pro-inflammatory marker. On the basis of these experiments, we treated HUVECs with $60 \mu \mathrm{M}$ of RVX208 followed by 12 hrs TNF- $\alpha$ treatment for BRD4 inhibition. As we did not find any difference in $4 \mathrm{hrs}$ and $12 \mathrm{hrs}$ treatment with JQ1 we used $500 \mathrm{nM}$ of JQ1 for 12 hrs followed by 12 hrs TNF-a treatment.

Comparative analysis indicated that 12 hrs pre-treatment with RVX208 reduces the expression of SELE by 2.8-fold and of VCAM-1 and IL6 by 3-fold and 7-fold, respectively, as compared to TNF- $\alpha$ treatment. However, pre-treatment with JQ1 reduces the expression of SELE by 6 -fold and of VCAM-1 and IL6 by 8 -fold and 10-fold, respectively, as compared to TNF- $\alpha$ treatment. This indicates that both JQ1 and RVX208 could efficiently inhibit the inflammatory response, however, JQ1 is rather more efficient than RVX208, and the reason could be non-specific interaction of JQ1 with BRD2 and BRD3, if any (Bid and Kerk, 2016; Huang et al., 2016; Huang et al., 2017; Wadhwa and Nicolaides, 2016; Zengerle et al., 2015). Interestingly, BRD4 is highly expressed in ECs as compared to BRD2 and BRD3 hence the results obtained with JQ1 might be directly attributable to BRD4 inhibition in these cells (Huang et al., 2016).

Another interesting observation from our treatments was whenever we treat the cells with RVX208-only or JQ1-only we observed the reduction of VCAM-I and IL6 expression as compared to DMSO control. RVX208-only treatment reduced the expression of VCAM-I 
and IL6 by 1.4-fold and 3-fold, respectively. Similarly, JQ1-only treatment reduces the expression of $V C A M-I$ and IL 6 by 2.5 -fold and 2.3 -fold, respectively. This data shows that BRD4 is also responsible for basal level gene expression of VCAM-I and ILG, therefore, in presence of only inhibitors basal level gene expression of these genes is also reduced. Brown et al. (2014) performed ChIP-Seq analysis and identified BRD4 binding on SEs of several pro-inflammatory genes including VCAM-I in presence and absence of TNF- $\alpha$.

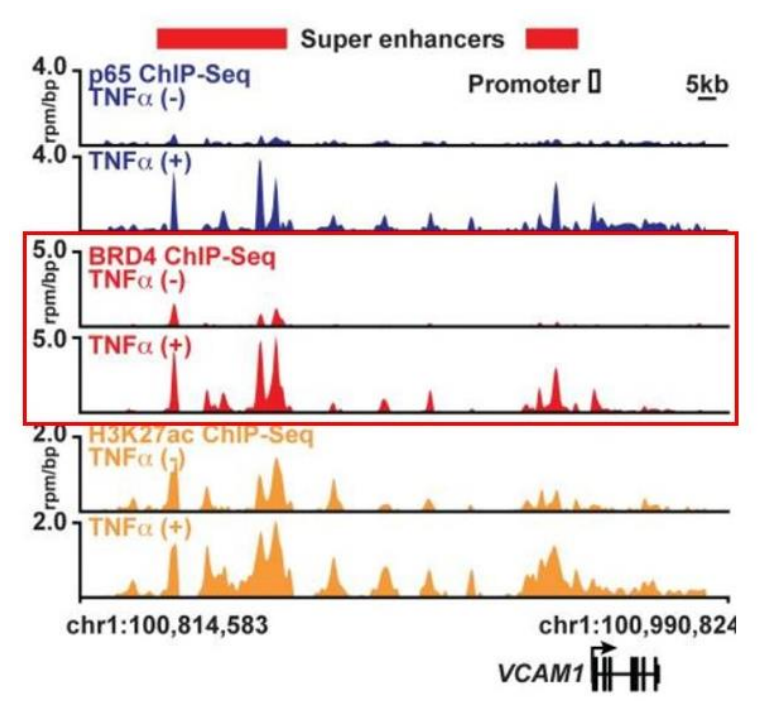

Figure 4.4 BRD4 genome binding in absence and presence of TNF- $\alpha$. Heat maps showing gene track ChIP-seq signals for BRD4 (red) at VCAM-I loci untreated (top) and TNF- $\alpha$ treated (bottom). (Figure published by Brown et al., 2014).

In normal resting ECs, BRD4 binds at VCAM-lenhancer region and that binding increases upon treatment with TNF- $\alpha$ (Fig. 4.4). This evidence shows that BRD4 binds to SEs region of VCAM-I, and perhaps also on IL6, in resting ECs. This might be the reason for the observed reduced expression of both genes in RVX208-only and JQ1-only treated cells.

BRD4 isoforms namely BRD4-long (BRD4-L) and BRD4-short (BRD4-S) are known for their differential roles in different diseases. In particular, over expression of BRD4-S is associated with enhanced metastasis in colon and breast cancers (Alsarraj et al., 2013; $\mathrm{Hu}$ et al., 2015). On the other hand, BRD4-L isoform has been reported to reduce metastasis (Alsarraj et al., 2013; Hu et al., 2015). Owing to these facts we were interested to determine the expression of BRD4 isoforms in normal as well as in inflammatory states of ECs. Hence, we studied the expression pattern of BRD4 isoforms not only in HUVECs 
in normal and inflammatory conditions, but also during BRD4 inhibition studies and in CVDs models like cardiac hypertrophy and aging hearts.

Our data indicated that mRNA expression of BRD4-total was high when HUVECs are treated with TNF- $\alpha$ for $12 \mathrm{hrs}$. We wanted to investigate further that the increased expression of $B R D 4$ during inflammatory stress is due to higher expression of $B R D 4-L$ or BRD4-S. We analyzed isoform specific mRNA expression and observed in case of inflammatory stimulus both isoforms have non-significant higher expression, however at protein levels we found short isoform to be significantly high during inflammatory stress. Moreover, we also observed BRD4-S high protein expression in cells which were pretreated with inhibitors followed by TNF- $\alpha$ treatment. As discussed previously that BRD4-S has been known for its role in enhancing cancer metastasis. Similarly, the observed increased expression of BRD4-S during inflammatory response in ECs may indicate its role in ECs dysfunction. However, further studies aimed at selective knock-down of BRD4 isoforms are necessary to confirm these results.

Similarly, when we treat cells with inhibitors-only we observed reduced expression of BRD4-total. These results indicate that BRD4 inhibitors not only inhibit the function of BRD4 but also reduce its expression suggesting auto-regulation of BRD4 expression. These results are in agreement with data of Wang et al. (2016) which reported suppression of BRD4 mRNA and protein expression in oral carcinoma cell line (Cal27), when treated with $500 \mathrm{nM}$ to $1 \mu \mathrm{M}$ of JQ1 (Wang et al., 2016). The reduced expression of BRD4-total in our system is due to reduced expression of BRD4- $L$ at mRNA and protein levels. We observed higher expression of $B R D 4-L$ in HUVECs, hence we may conclude that JQ1 and RVX208 both effect BRD4-L levels in HUVECs probably because of its higher expression.

Aging is a major non-modifiable risk factor, which contributes in progression of CVDs. Agespecific cardiac remodelling includes left ventricle hypertrophy, fibrosis, cardiomyocytes lengthening, and deposition of ECM in cardiac tissues leads to multiple pathological cardiac conditions (Cheng et al., 2009; Mottonen et al., 2017). One interesting hallmark of age specific cardiac remodelling is isoform shift of Myosin heavy chains in heart. Embryonic heart mainly consists of $\beta-\mathrm{MHC}$ which converts to $\alpha-\mathrm{MHC}$ in adult heart (Gupta, 2007; Mahdavi et al., 1984). As the mammals age, $\beta$-MHC became dominant again (Gupta, 2007). Similarly, in cardiac hypertrophy, $\alpha-M H C$ disappears, and $\beta-M H C$ is dominantly expressed (Gupta, 2007; Herron et al., 2001; Hudson et al., 2011). Keeping these changes 
in mind, we decided to analyse whether BRD4 isoforms have any role during cardiac remodelling due to aging and cardiac hypertrophy. The mRNA expression analysis of Brd4total, Brd4-L and Brd4-S did not show any significant differences in mice hearts of 1 to 18months-old except for 6-months-old mice heart, which showed significantly higher Brd4-S expression. Protein expression of BRD4-S was only detectable till 8-months old and BRD4$L$ was significantly higher in 18-months-old mice hearts. However, further analysis on older than 18 months could further explain that whether BRD4-L isoform is high at old age or not. It is known that there is an increase in expression of inflammatory genes in pressure overloaded hearts (Chen et al., 2015; Souders et al., 2012). BRD4 inhibition reduces the expression of pro-fibrotic genes and have been recommended as a treatment of fibrosis (Stratton et al., 2017). In this context, we decided to analyse the expression of BRD4 protein in cardiac fibrosis model of TAC mice. Our data showed that there is no significant expression difference in TAC and SHAM mice. This data may indicate that BRD4 isoforms expression may not vary during cardiac remodelling of hearts in hypertrophy or during aging process.

ECs monolayer is classified as largest organ due to its active contribution in physiological pathways (Aird, 2004; Anggard, 1990). It acts as a barrier between blood and tissues and regulates immune system, transport of nutrients and growth of new vessels by secreting biologically active substances (Aird, 2004; Anggard, 1990). Inflammation, microbial infection, ROS and increased oxidative stress due to oxidized LDL in blood can cause ECs monolayer disruption and which can lead to progression of multiple diseases including atherosclerosis (Ji et al., 2015).

We decided to analyse the effect of inflammatory stimulus on ECs barrier function in-vitro. HUVEC monolayers were established and were first treated with TNF- $\alpha$ to analyse the effect of inflammatory stimulus. We observed reduction in trans endothelial electric resistance, increase in permeability across the monolayer with 12 hrs of TNF- $\alpha$ treatment. These results support a previous study of Urbano et al. (2017), which reported increase of permeability across ECs with TNF- $\alpha$ treatment. Our data also indicate that whenever HUVECs were pre-treated with RVX208 or JQ1 followed by TNF- $\alpha$ treatment inhibited the barrier dysfunction. These results indicate that BRD4 inhibition is reducing the expression of pro-inflammatory cytokines and adhesion molecules and protecting the ECs monolayer from disruption. 
Huang et al. (2016) previously reported that JQ1 treatment reduced the VEGF-induced permeability by blocking p21-activated kinase-1 (PAK-1) and eNOS activation. From literature review we know that VEGFR2 is also responsible for activation of p38MAPK pathway and in line with this, Huang et al. (2017) in another study reported that JQ1 treatment suppresses vascular inflammation via blocking of p38MAPK pathway. Interestingly, our group previously reported increased permeability in human epithelial colorectal adenocarcinoma cells (Caco-2 cells) is due to a cytokine Midkine, an upstream regulator of p38MAPK pathway (Khan et al., 2017). It has been reported that cytokines can modulate the cytoskeleton of ECs and open the gaps between adjacent cells which increase the permeability of ECs monolayers (Ramji and Davies, 2015). Among different cytokines, Midkine is an interesting target as it has been reported to be highly expressed in inflammatory kidney disease and atherosclerosis (Salaru et al., 2013; Salaru et al., 2016).

Based on these evidences, we decided to analyse Midkine expression in ECs monolayer in the presence of inflammatory stimuli. Our data showed increased expression of MIDKINE at mRNA level after 6 hrs of TNF- $\alpha$ treatment and it steadily increased until $72 \mathrm{hrs}$ of treatment. At protein level we observed MIDKINE to be secreted from HUVECs after 36 hrs of TNF- $\alpha$ treatment and it steadily increased until $72 \mathrm{hrs}$ of treatment. This data is in agreement with the higher expression of Midkine during inflammatory response which is responsible for increased permeability (Fig. 4.5) (Salaru et al., 2013; Salaru et al., 2016). In conclusion, our data shows ECs monolayer disruption is due to increased expression of MIDKINE. This data is in agreement with previous studies which reported the increased expression of MIDKINE in atherosclerotic plaques (Salaru et al., 2013). 


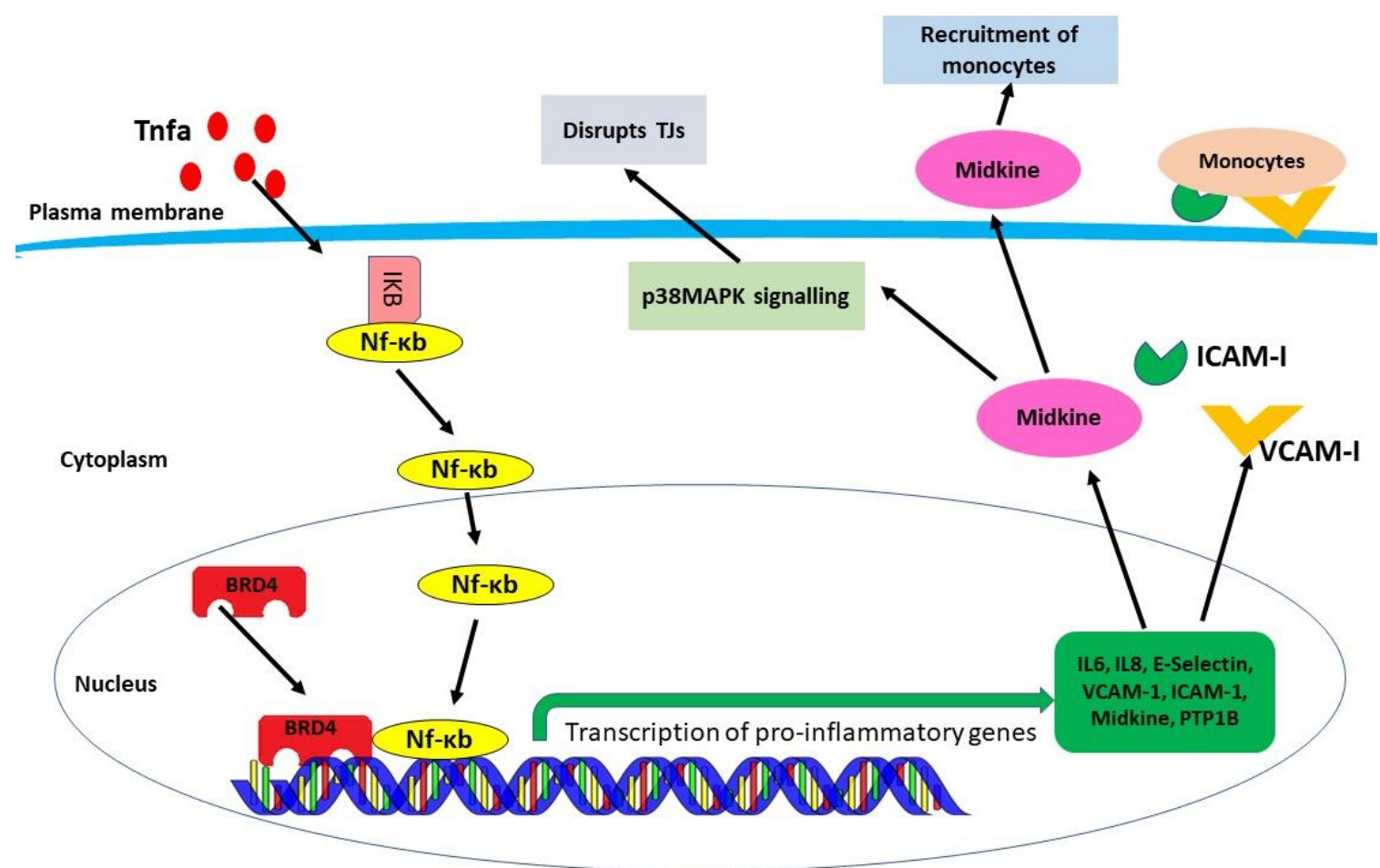

Figure 4.5. Summary of inflammatory response in ECs, Inflammatory stimuli (TNF- $\alpha$ ) induces activation and translocation of NF-kB into nucleus. NF-KB along with BRD4 protein transcribe pro-inflammatory markers including 1: ICAM, VCAM-I, which express on ECs monolayer and bind to monocytes from blood 2: MIDKINE, which contributes in recruitment of monocytes and activates p38MAPK mediated disruption of tight junctions in ECs and increase the permeability of ECs monolayer.

As our data showed that BRD4 inhibition is protecting ECs monolayer from inflammation and protecting monolayer integrity. We were interested to analyse what is the effect of BRD4 inhibition on expression of Midkine. Our data shows that pre-treatment with RVX208 or JQ1 significantly reduces the expression of Midkine at mRNA and protein levels. On the basis of these results we concluded that BRD4 inhibition not only protecting ECs from inflammatory phenotype but also protecting their barrier function via reducing the expression of Midkine (Fig. 4.6). Taken together, further animal studies should be conducted to analyses effect of BRD4 in-vivo to decide whether BRD4 inhibition could be used as therapeutic approach in treatment of atherosclerosis and other inflammatory diseases. 


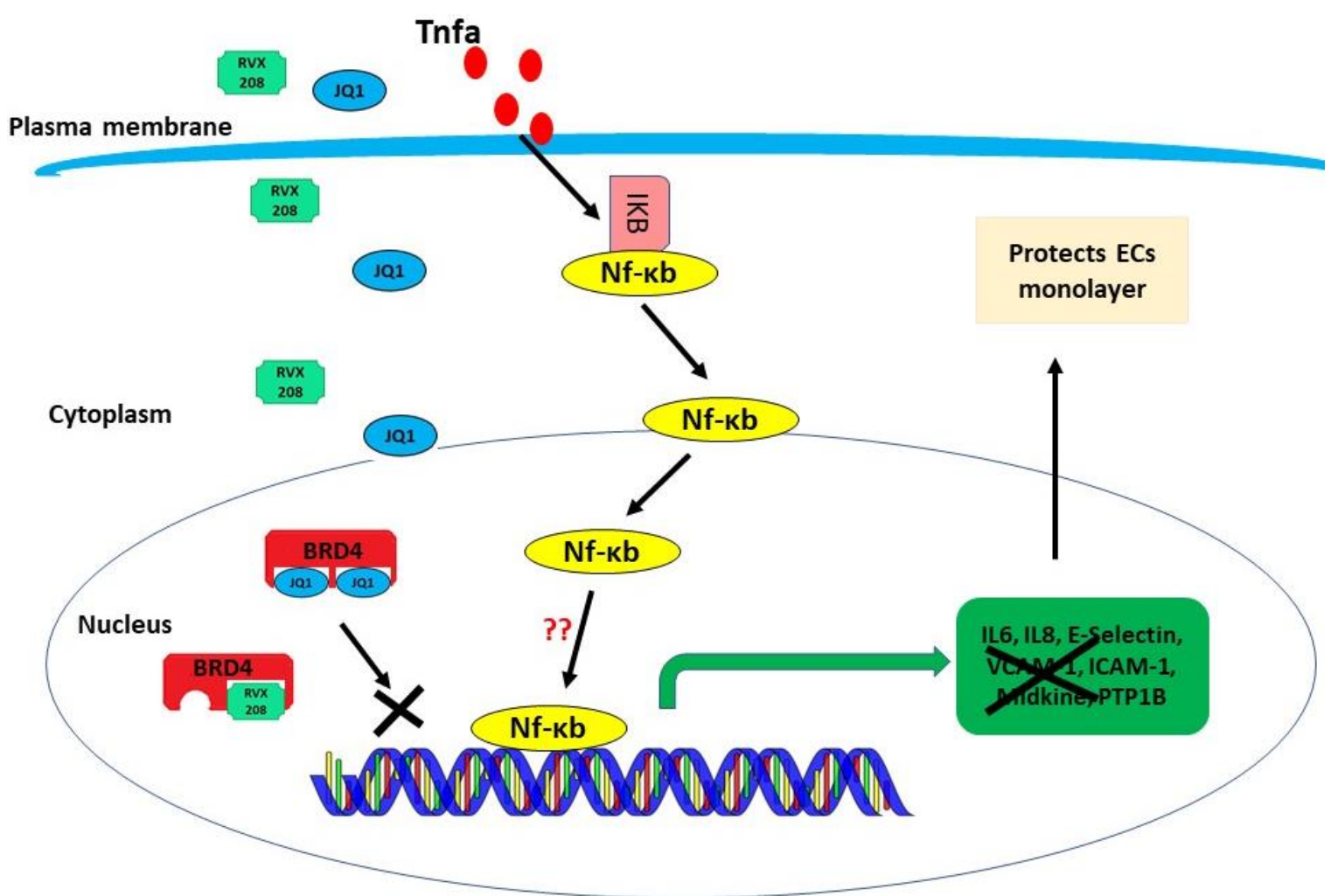

Figure 4.6 BRD4 inhibition reduces the expression of pro-inflammatory markers: Small molecular inhibitors of BRD4 binds to BRD4 bromodomains and inhibits its binding to chromatin, which reduces the expression of proinflammatory markers and protects ECs from activation and tight junction disruption.

Next, we wanted to study the role of PTP1B, a target of BRD4 and NF-kB pathway and it has been reported to be overexpressed during inflammation (Zabolotny et al., 2008). PTP1B is a phosphatase which is ubiquitously expressed in mammalian tissues and is responsible for activation and deactivation of multiple signalling pathways (Zabolotny et al., 2008). PTP1B dephosphorylates insulin receptor, EGFR/VEGFR receptor, platelet derived growth factor receptor (PDGFR), Colony stimulating factor 1 receptor (CSFR-1), and insulin growth factor receptor (IGFR) (Fig. 4.7). In ECs, PTP1B overexpression leads to ECs dysfunction, inhibition of VEGFR2 signalling pathway resulting in impaired ECs migration and angiogenesis (Lanahan et al., 2014; Thiebaut et al., 2016; Zabolotny et al., 2008). PTP1B inhibition protects the mice from developing atherosclerotic plaques (Thompson et al., 2017). 


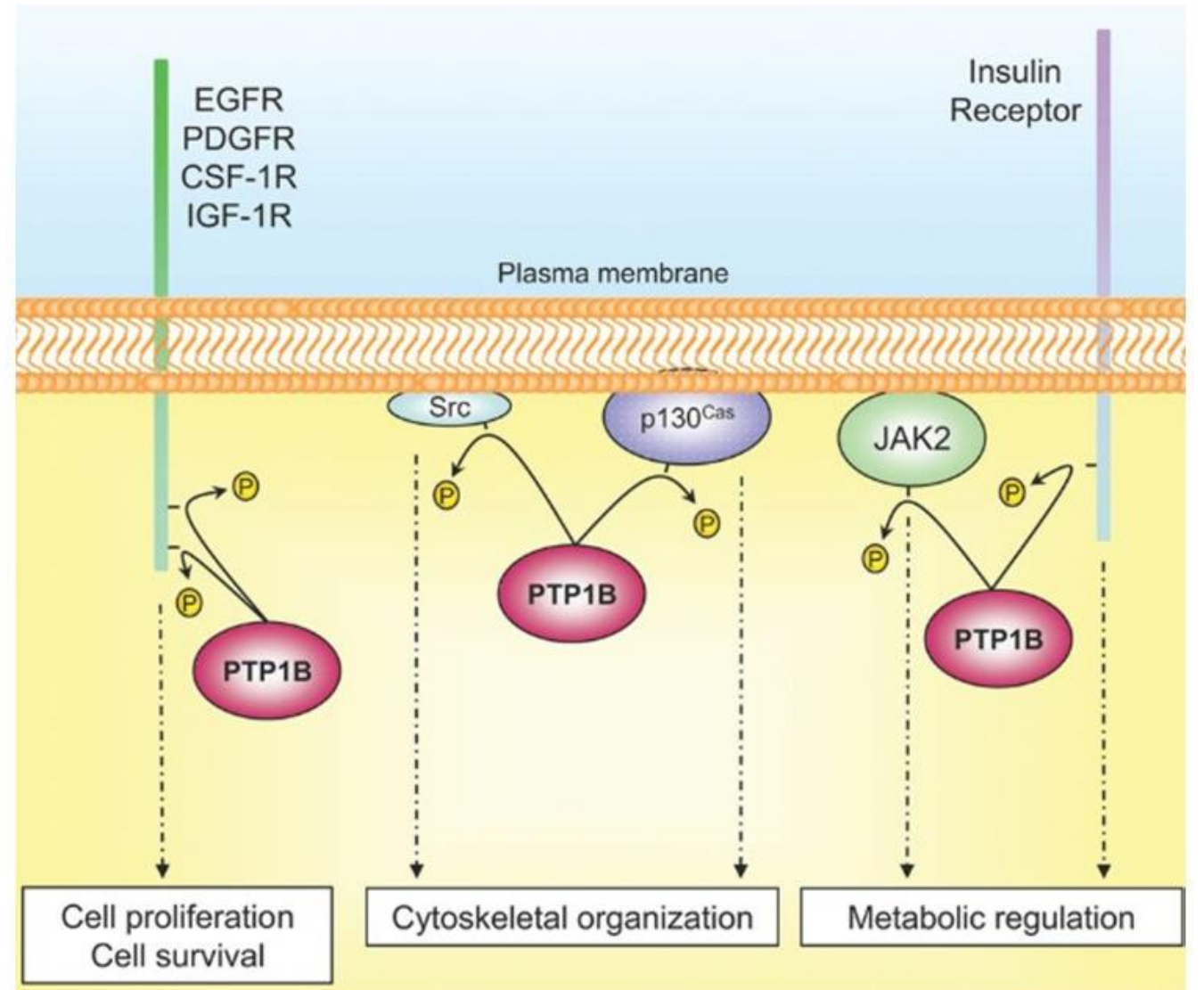

Figure 4.7 PTP1B target receptors: PTP1B dephosphorylates insulin receptor, VEGFR receptor, PDGFR, CSF1R, IGF-1R receptor and regulates metabolic pathways, cytoskeleton organization, cell proliferation and survival. (Figure published by Tiganis et al., 2007).

We observed significantly higher expression of PTP1B gene and protein in HUVECs, when treated with TNF- $\alpha$. These results are in agreement with previous studies on muscle myoblasts cell line (C2C12) and adipose tissues, which showed over expression of PTP1B when treated with TNF- $\alpha$ (Panzhinskiy et al., 2013; Zabolotny et al., 2008).

PTP1B inhibition studies have shown it as a promising approach in treatment of obesity, diabetes, hypertension, ECs dysfunction, cardiac dysfunction, and cardiac inflammation (Ali et al., 2009; Bruder-Nascimento et al., 2015; Coquerel et al., 2014). A recent study by Gogiraju et al., 2016 showed ECs specific deletion of PTP1B (Endo.Ptp1b.KO) protects the mice from cardiac hypertrophy and cardiac fibrosis in TAC model (Gogiraju et al., 2016). However, it was intriguing to further investigate the proteins and pathways which were affected in Endo.Ptp1b.KO heart tissues. To answer this, we performed a quantitative global proteome analysis on wild type and Endo.Ptp1b.KO mice with and without induced pressure via aortic constriction. 
Our proteomic analysis could identify 899 proteins in all four groups (WT-SHAM, WT-TAC, KO-SHAM, KO-TAC) with differential expression, however with 1\% FDR we could quantify and consider only 697 proteins for further analysis. Previously, proteomic studies on TAC mice reported 538 proteins (Dai et al., 2013). As per our knowledge this is the first study to show cardiac proteome analysis of ECs with Ptp1b specific deletion.

Our data showed only 8 up-regulated and 58 down-regulated proteins in KO-SHAM as compared to WT-SHAM. Pathway analysis showed that all these proteins do not belong to one specific pathway but included interesting targets like Gap junction alpha-1 protein, Calnexin, ribosomal proteins, Glutathione oxidase and cytoskeleton proteins. Gap junction alpha-1 protein (GJA1), also known as connexin43, is one of the major cardiac gap junction protein and a marker of cardiac lineage (Eshkiki et al., 2017; Kurtenbach et al., 2014). It has been reported that PTP1B knock down results in overexpression of GJA1 which interrupts the embryonic stem cells differentiation towards cardiac lineage (Eshkiki et al., 2017). Interestingly, a previous study has reported that reduced expression of GJA1 is a marker of congestive heart failure (Kurtenbach et al., 2014). In line with these previous observations, our data showed 2-fold up-regulation of GJA1 in KO-SHAM as compared to WT-SHAM which may indicate improved cardiac gap junctions in Ptp1b KO animals. Calnexin is an endoplasmic reticulum (ER) membranous protein which is responsible for folding of secretory and membrane bounded proteins (Lee et al., 2015). It has been reported that Calnexin forms a complex with C-terminal of PTP1B and act as an anchor of PTP1B in ER (Lee et al., 2015). We observed a 2-fold decreased expression of Calnexin in KO-SHAM. This data suggests that KO of PTP1B leads to down-regulation of Calnexin through unknown mechanism. Moreover, it has also been reported that Calnexin-PTP1B interaction recruits PTP1B to ribosome-translocon complex ensuring its post-translation modification role on various targets (Lee et al., 2015). Ribosome translocon complex includes ribosomal subunits like 60S and 40S (Voorhees et al., 2014). Interestingly we observed reduced expression of multiple ribosomal protein of $60 \mathrm{~S}$ subunit (Proteins: P1, L4, L27A and L29) and of 40S subunit (S4, S9 and S16) up to 1.5-fold to 2-fold. However, we did not find any translocon protein to be down-regulated. Oxidative stress leads to inactivation of PTP1B via its oxidation (Geraghty et al., 2013). Glutathione peroxidase (Gpx) is a common intracellular antioxidant enzyme which has higher activity in response to inflammatory stimuli and oxidative stress (Geraghty et al., 2013; Mobasher et al., 2013). It has been reported that Gpx-activity did not increased in PTP1B deficient hepatocytes 
during induced hepatotoxicity and cells show increased antioxidant defence (Mobasher et al., 2013). We observed 2-fold down-regulation of Gpx in KO-SHAM tissues which may indicate that Ptp1b deletion protects the hearts against ROS stress. PTP1B is known to regulate adhesion and migration of cells via dephosphorylation of cytoskeleton proteins like Actin, Filamin C, Cofilin and Fibrinogen (Arregui et al., 2013; Sumi et al., 1999; Wang et al., 2018). Cellular movements depend on polymerisation and depolymerisation of actin cytoskeleton (Sumi et al., 1999). VEGFR phosphorylation leads to activation of p38MAPK pathway which induces actin polymerisation via phosphorylates of cofilin, an acting binding protein (Wang et al., 2018). PTP1B is a regulator of VEGFR signalling and PTP1B deletion from ECs leads to increased phosphorylation of VEGFR (Gogiraju et al., 2016; Lanahan et al., 2014). Interestingly we found that in absence of PTP1B multiple cytoskeleton proteins are more than 2-fold down-regulated including fibrinogen and actin related proteins: cofilin and filamin C. This data shows that absence of PTP1B may not only affect the cytoskeleton proteins function but also might affect their expression. Hence, further studies are important to answer these observations.

Next, we analysed WT-SHAM and WT-TAC proteins and our data showed 60 up-regulated and 70 down-regulated proteins in WT-TAC as compared to WT-SHAM. Multiple previous studies analysed TAC hearts for global proteome, however, we reported highest number of differentially regulated proteins in WT-TAC mice which is 130 proteins as compared to previously reported 123 (proteomics study) and 95 (transcriptomics + proteomics study) (Dai et al., 2013; Lau et al., 2018). Pathway analysis showed that up-regulated proteins exhibit one cluster of cytoskeleton proteins. It has been reported previously that pressure overloaded hearts exhibit increased density of microtubules component which contributes to stiffness of cardiac muscle (Dai et al., 2013; Lau et al., 2018; Tagawa et al., 1997). We observed some interesting cytoskeleton proteins in WT-TAC, including Myosin heavy chain, Four and a half LIM domains 1 (FHL1) and $\beta$-Tubulin, to be up-regulated.

Myosin is a main molecule of thick filament of cardiac muscle which generates movement by hydrolysis of ATP (Gupta, 2007). Myosin consists of 2 heavy chains (MHCs) (encoded by gene $\alpha$ and $\beta$ ) and four light chains (MLCs) (Gupta, 2007; Mahdavi et al., 1984). In rodents $\beta-\mathrm{MHC}$ expresses predominantly in ventricles and before birth, expression of $\alpha$ MHC starts to increase in ventricles and dominates in young rodents (Gupta, 2007). Isoform shift of Myosin from $\alpha-\mathrm{MHC}$ to $\beta-\mathrm{MHC}$ is an important hallmark of hypertrophy (Gupta, 2007; Herron et al., 2001; Hudson et al., 2011). We observed 22-fold up-regulation 
in $\beta-M H C$ in WT-TAC as compared to WT-SHAM further validating our proteome analysis. Four and a half LIM domain proteins domain (FHL) proteins, such as FHL1 are nonenzymatic proteins associated with cytoskeleton (McGrath et al., 2006). Higher expression of FHL1 at transcriptome and proteome level is considered as a marker of cardiac hypertrophy (Dai et al., 2013; Lau et al., 2018). We observed 4-fold up-regulation of FHL1 in WT-TAC mice as compared to WT-SHAM. On the other hand Tubulin protein is a dimer of $\alpha$ - and $\beta$-tubulin and it has been reported that $\beta$-tubulin expression is high in human failing hearts with cardiac hypertrophy (Aquila-Pastir et al., 2002). We also observed 2.4fold higher expression of $\beta$-tubulin in hypertrophic TAC mice.

Interestingly in down-regulated proteins of WT-TAC, we observed one major cluster consisting of mitochondrial associated proteins. Previous studies have also reported that pressure overloaded hearts have mitochondrial dysfunction due to down-regulation of oxidation-reduction associated proteins, fatty acid metabolism proteins and electron transport chain associated proteins (Dai et al., 2013; Lau et al., 2018). Mitochondrial dynamics change during cardiac remodelling in CVDs (Vasquez-Trincado et al., 2016). Myocardial infarction, atherosclerosis, diabetic cardiomyopathy and cardiac hypertrophy poses increased energy demand stress on mitochondria in cardiac tissue which leads to cell death, mitochondrial DNA damage, and metabolic disorder, respectively (Fig. 4.8) (Vasquez-Trincado et al., 2016). 


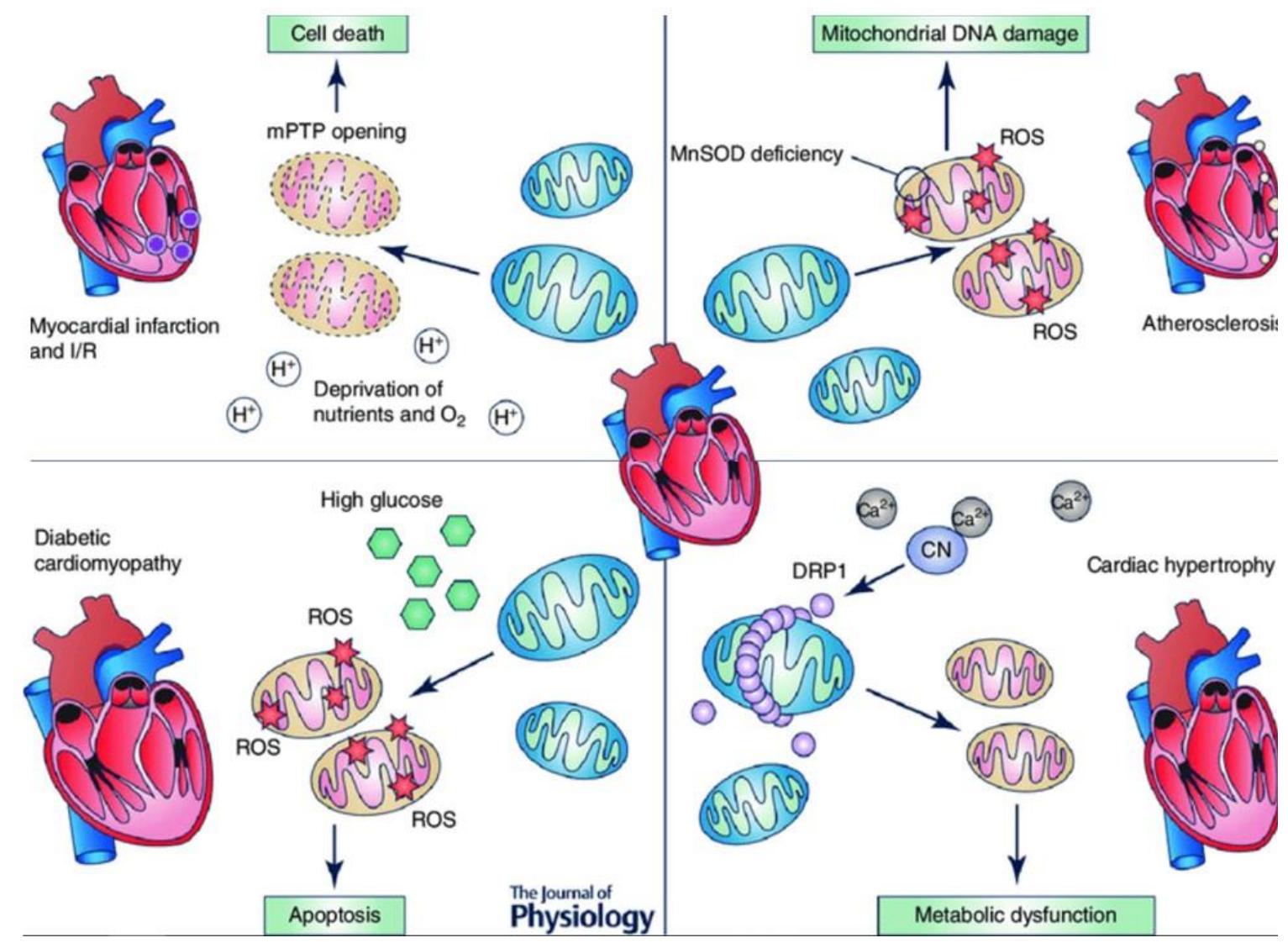

Figure 4.8 Mitochondrial dysfunction in CVDs: CVDs leads to extensive cardiac remodelling and metabolic stress on cardiac tissues. In myocardial infarction cardiac cells are deprived of oxygen and nutrients which leads to mitochondrial permeability transition pore opening ( (MPTP) causing cell death. Cardiac tissues face higher ROS in atherosclerosis which cause mitochondrial DNA damage. In diabetic cardiomyopathy, apoptosis is high due to increased ROS production. In cardiac hypertrophy, pressure overload leads to increase demand of mitochondrial biogenesis and energy production and failure of it leads to increase demand of mitochondrial biogenesis and energy production and failure leads to metabolic dysfunction in hypertrophic hearts (Figure published by Vasquez et al., 2016).

Previously, proteomic study and IPA analysis on pressure overload heart showed attenuated mitochondrial function, oxidative phosphorylation and Rho based actin cytoskeleton polymerisation (RhoGDI signalling) and Rho GTPase signalling (Dai et al., 2013). In support of these findings our WT-TAC data after IPA analysis showed similar results (Fig.4.9). We observed attenuated pathways including oxidative phosphorylation ( $\mathrm{p}$ value $5.19 \mathrm{e}^{-05}$ ), mitochondrial dysfunction ( $p$ value $1.97 \mathrm{e}^{-04}$ ), RhoGDI signalling ( $p$ value $2.19 \mathrm{e}^{-04}$ ), and Rho Family GTPases signalling ( $p$ value $6.15 \mathrm{e}^{-04}$ ) (Fig. 4.9).

Furthermore, IPA analysis revealed a list of possible phenotypic symptoms on the basis of differentially regulated proteins which includes cardiac arrythmia, dilation, heart failure, cardiac fibrosis and cardiac infarction (Fig. 4.11). Interestingly these phenotypic symptoms 
resemble to TAC induced cardiac hypertrophy in WT mice hearts. This data also validates the hypertrophy in WT mice.

\begin{tabular}{lc} 
Summary of Analysis - Observation 2 & \\
\hline Top Canonical Pathways & \\
\hline Name & p-value \\
\hline Oxidative Phosphorylation & $5.19 \mathrm{E}-05$ \\
Mitochondrial Dysfunction & $1.97 \mathrm{E}-04$ \\
RhoGDI Signaling & $2.19 \mathrm{E}-04$ \\
Signaling by Rho Family GTPases & $6.15 \mathrm{E}-04$ \\
Glycerol-3-phosphate Shuttle & $2.73 \mathrm{E}-03$ \\
Cardiotoxicity & \\
Name & p-value \\
\hline Cardiac Arrythmia & $1.43 \mathrm{E}-02-6.83 \mathrm{E}-04$ \\
Cardiac Dilation & $3.96 \mathrm{E}-02-1.09 \mathrm{E}-03$ \\
Heart Failure & $9.53 \mathrm{E}-03-9.53 \mathrm{E}-03$ \\
Cardiac Fibrosis & $1.56 \mathrm{E}-02-1.56 \mathrm{E}-02$ \\
Cardiac Infarction & $1.70 \mathrm{E}-01-1.70 \mathrm{E}-01$ \\
\hline
\end{tabular}

Figure 4.9 IPA analysis of WT-TAC proteins compared to WT-SHAM proteins: IPA analysis showing top canonical pathways attenuated in WT-TAC (upper panel) which includes oxidative phosphorylation, mitochondrial dysfunction, RhoGDI signalling and RhoGTPases signalling. IPA analysis also revealed cardiotoxic effect of these proteins and pathways in WT-TAC resembling to hypertrophic heart properties (lower panel).

Further on we analysed which proteins could be more significantly differentially regulated in our proteome data causing above mentioned mitochondrial dysfunction. In support of this hypothesis, we observed in our down-regulated protein data some mitochondrial associated proteins which have been previously reported to be down-regulated during pressure overload. NADH dehydrogenase is a largest enzyme complex consisting of almost 44 subunits and functions in mitochondrial Electron Transport Chain (ETC). It has been reported that NADH dehydrogenase 1 - $\beta$ subcomplex subunit 4 is down-regulated in pressure overloaded failing hearts (Bugger et al., 2010). Interestingly, we found also this protein to be 2.3-fold downregulated in our WT-TAC samples. Moreover, we found also another subunit, NADH dehydrogenase 1 - $\beta$ subcomplex subunit 1 , to be down-regulated up to 2 -fold.

ATP synthase subunit delta is a subunit of mitochondrial ATP synthase enzyme which is responsible for ATP synthesis and is reported to be downregulated in failing hearts (Bugger 
et al., 2010). We observed 2.2-fold down-regulation of ATP synthase subunit delta in WTTAC. It has been reported that pressure overloaded hearts exhibit down-regulation of multiple fatty acid $\beta$ oxidation pathway proteins at transcriptome and proteome level (Lau et al., 2018). We observed multiple fatty acid metabolism associated enzymes to be downregulated in WT-TAC mice including 3-ketoacyl-CoA thiolase (2.79-fold), MethylcrotonoylCoA carboxylase (2.62-fold), Carboxylesterase 1 (2.5-fold), Enoyl-CoA delta isomerase 1 (2.3-fold) and biosynthesis O-methyltransferase (2.02-fold). These findings show that WTTAC had impaired fatty acid metabolism, yet another hallmark of hypertrophy (Lau et al., 2018). Collectively, our data showed down-regulation of above-mentioned proteins which have been reported previously in WT-TAC mice. However, we observed many other mitochondrial associated proteins which have not been reported previously such as ATP binding cassette protein (3.34-fold), Phospholemman (2.12-fold), Sodium/calcium exchanger 1 (2.09), Glutaryl-CoA dehydrogenase (1.86-fold), Acyl-coenzyme A thioesterase 2 (1.86-fold), Enoyl-CoA hydratase (1.77-fold), Hydroxyacyl-coenzyme A dehydrogenase (1.76-fold), Acetyl-coenzyme A synthetase (1.55-fold) etc. Together with the known mitochondrial proteins, all these down-regulated proteins confirm that WT-TAC mice have mitochondrial dysfunction and impaired metabolic pathways.

Table 4.1 shows the comparison of some published studies with our proteomic study, we observed similar pattern of proteins in WT-TAC as published already in different proteomic studies.

Table 4.1. Comparison of our proteomic results with different published LC-MS/MS based proteomic studies on TAC animals

\begin{tabular}{|c|c|c|c|c|c|}
\hline & $\begin{array}{l}\text { Dao-Fu Dai } \\
\text { et al., } 2013\end{array}$ & $\begin{array}{l}\text { Dao-Fu Dai } \\
\text { et al., } 2012\end{array}$ & $\begin{array}{l}\text { Rüdebusch } \\
\text { et al., } 2017\end{array}$ & $\begin{array}{l}\text { Bugger et al., } \\
2009\end{array}$ & Our study \\
\hline Animal model & C57BL6 mice & C57BL6 mice & C57BL6 mice & $\begin{array}{l}\text { Sprague-Dawley } \\
\text { Rat }\end{array}$ & C57BL6 mice \\
\hline $\begin{array}{l}\text { Heart collection } \\
\text { after surgery } \\
\text { (days) }\end{array}$ & 28 & 28 & 56 & 140 & 140 \\
\hline Sample type & Left ventricle & Purified & Left ventricle & Mitochondria & Heart lysates \\
\hline
\end{tabular}




\begin{tabular}{|c|c|c|c|c|c|}
\hline & & mitochondria & & & \\
\hline Search engine & SEQUEST & SEQUEST & SEQUEST & $\ldots \ldots$. & MASCOT \\
\hline Statistical test & $\begin{array}{l}\text { Two-way } \\
\text { ANOVA }\end{array}$ & $\begin{array}{l}\text { Two-way } \\
\text { ANOVA }\end{array}$ & $\begin{array}{l}\text { Two-way } \\
\text { ANOVA }\end{array}$ & T-test & T-test \\
\hline $\begin{array}{l}\text { P-value/ } \\
\text { Fold change }\end{array}$ & $0.05 / 1.5$-fold & $0.05 / 1.5$-fold & 0.05/2-fold & $0.05 />0.5$-fold & $0.05 / 1.5$-fold \\
\hline $\begin{array}{l}\text { Number of } \\
\text { differentially } \\
\text { regulated } \\
\text { proteins }\end{array}$ & 121 & 96 & 99 & 70 & 130 \\
\hline $\begin{array}{l}\text { Canonical } \\
\text { Pathways }\end{array}$ & $\begin{array}{l}\text { Actin signalling } \\
\text { pathway, Rho } \\
\text { signalling, Fatty } \\
\text { acid, Glycolysis, } \\
\text { Oxidative } \\
\text { phosphorylation }\end{array}$ & $\begin{array}{c}\text { ETC, } \\
\text { Transmembrane } \\
\text { transport, ATP } \\
\text { synthesis, protein } \\
\text { folding }\end{array}$ & $\begin{array}{l}\text { Actin signalling } \\
\text { pathway, Rho } \\
\text { signalling, Fatty } \\
\text { acid, Oxidative } \\
\text { phosphorylation }\end{array}$ & $\begin{array}{c}\text { Fatty acid, } \\
\text { Oxidative } \\
\text { phosphorylation }\end{array}$ & $\begin{array}{l}\text { Actin signalling } \\
\text { pathway, Rho } \\
\text { signalling, Fatty } \\
\text { acid, Glycolysis, } \\
\text { Oxidative } \\
\text { phosphorylation }\end{array}$ \\
\hline $\begin{array}{l}\text { Mitochondrial } \\
\text { proteins }\end{array}$ & Downregulated & $\ldots$. & $\ldots \ldots$. & Downregulated & Downregulated \\
\hline
\end{tabular}

Next, we analysed 2 groups having PTP1B deficiency, namely SHAM and TAC. Previously, Gogiraju et al., 2016 reported reduced fibrosis and hypertrophy in KO-TAC. We observed different proteome in KO-TAC as compared to KO-TAC and we found 150 up-regulated and 53 downregulated proteins. Pathway analysis showed that up-regulated proteins includes mitochondrial proteins, proteasome associated proteins and proteins involved in secretion and transportation. In down-regulated proteins we observed small clusters of mitochondria, cytoskeleton, and ribosomal proteins.

As mentioned above WT-TAC mice having higher expression of $\beta-\mathrm{MHC}$ (up to 22-fold) is a major hallmark of cardiac hypertrophy. Unexpectedly, we also observed increased expression of $\beta$-MHC (up to 7-fold) in KO-TAC, however, this up regulation is 3-fold less as compared to WT-TAC. Even though PTP1B deficiency in mice is protecting from cardiac fibrosis and hypertrophy, still TAC surgery might be inserting pressure overload on cardiac tissues. KO-TAC showed up-regulation of multiple mitochondrial associated proteins as 
compared to SHAM mice. TAC surgery exhibits pressure on cardiac tissues which leads to increased mitochondrial biogenesis to compensate the energy demand (Rosca et al., 2013). However hypertrophic hearts exhibit reduced mitochondrial biogenesis which leads to cellular death (Rosca and Hoppel, 2013). Our data suggest that KO hearts have increased mitochondrial biogenesis and have balance between energy demand and production. KO-TAC mice hearts also showed over-expression of proteasome associated proteins. Proteasomal over-expression can be related to over-expression of proteins in TAC mice to degrade misfolded proteins. However, it has also been reported that downregulation of PTP1B improves the proteasomal function in neuroblastoma cell lines (Jeon et al., 2017). So, over-expression of proteasomal subunits could be an effect of PTP1B deficiency, in general.

PTP1B-deficient mice hearts are protected from hypertrophy and fibrosis as compared to WT-TAC. Proteome analysis between WT-TAC and KO-TAC mice showed 43 upregulated and 36 down-regulated proteins in KO-TAC as compared to WT-TAC. STRING analysis showed mitochondrial cluster in up-regulated proteins while in down-regulated proteins showed cluster of ribosomal and cytoskeleton proteins. This data is quite interesting and related to previous discussion that KO-TAC showed less hypertrophy and fibrosis as compared to WT-TAC. Mitochondrial dysfunction is associated with pressure overload in human and mice hearts (Dai et al., 2013; Dai et al., 2012; Lau et al., 2018). Our data showed PTP1B deficiency leads to improved mitochondrial function via increased expression of mitochondrial proteins.

Previously 10 subunits of NADH were found downregulated in WT-TAC mice (Dai et al., 2012). We observed 9 subunits of NADH dehydrogenase to be up-regulated in KO-TAC. We observed some other mitochondrial up-regulated proteins like 3 subunits of Cytochrome b-c1 complex, 3-ketoacyl-CoA thiolase, Cytochrome c oxidase, ATP-synthase subunit delta, Hydroxyacyl-coenzyme $A$ dehydrogenase and $\mathrm{NADH}$-ubiquinone oxidoreductase.

Some other interesting up-regulated proteins include $26 \mathrm{~S}$ proteasome complex, which have been reported to protect mice from cardiac hypertrophy (Dickhout and Austin, 2006). We also observed up-regulation of Sepiapterin reductase, a crucial enzyme in (6R)-Ierythro-5,6,7,8-tetrahydrobiopterin (BH4) biosynthesis (Sumi-Ichinose et al., 2017). BH4 is an important cofactor of NOS enzyme and it reverses the oxidative stress, cardiac 
hypertrophy, and fibrosis in a 5-week-old pressure induced hypertrophy model (Moens et al., 2008; Sumi-Ichinose et al., 2017). Overexpression of Sepiapterin reductase in KO-TAC indicates that PTP1B deficiency in ECs may improving the eNO production which is contributing in reducing the oxidative stress, fibrosis, and hypertrophy. FHL1 a marker of cardiac hypertrophy and was up to 1.77-fold downregulated in KO-TAC as compared to WT-TAC. Glucose transporter 4 (GLUT4) is responsible for glucose uptake and deficiency of it leads to cardiac hypertrophy (Wende et al., 2017). We observed up-regulation of GLUT4 in KO-TAC mice as compared to WT-TAC showing improved glucose metabolism in KO-TAC.

Among down-regulated proteins, we identified multiple ribosomal proteins. As discussed previously that PTP1B deficiency leads to reduced expression of ribosomal proteins. However, as PTP1B deficient hearts have reduced fibrosis and hypertrophy, this might be the reason that there is reduced protein biosynthesis machinery. KO-TAC mice also showed down-regulation of cytoskeleton associated proteins, notably $\beta-\mathrm{MHC}$, a hallmark of hypertrophy, was down-regulated in KO-TAC as compared to WT-TAC. Elevated levels of Integrin linked kinase, that physically links $\beta$-integrins with the actin cytoskeleton, have been reported in human cardiac hypertrophy and induces hypertrophy in mice (Lu et al., 2006). In line with these observations, we found up to 3-fold down-regulation of integrin linked kinase in KO-TAC as compared to WT-TAC. It has been reported that there is an increase in transcription of $\beta 1$ - and $\beta 2$-tubulin genes in cardiac hypertrophy (Narishige et al., 1999). We observed 2.98-fold down-regulation of Tubulin- $\beta$ in KO-TAC as compared to WT-TAC. Other than cytoskeleton associated proteins we also observed that KO-TAC mice have decreased expression Carbonic Anhydrase I and II (CA-I and CA-II) as compared to WT-TAC. Carbonic Anhydrases (CAs) are a group of enzymes which are responsible for the reversible conversion of $\mathrm{CO}_{2}$ and $\mathrm{H}_{2} \mathrm{O}$ in $\mathrm{HCO}_{3}{ }^{-}$and $\mathrm{H}^{+}$. Inhibition of CAs in rats showed reduced hypertrophy. CA-II expression is high in cardiac hypertrophic mice (Alvarez et al., 2013). Interestingly, human hypertrophic ventricles show increased expression of CA-II and CA-IV expression (Alvarez et al., 2013). In our study we found that KO-TAC mice have decreased expression CA-I and CA-II as compared to WT-TAC suggesting Carbon metabolism is balanced in KO-TAC model. 


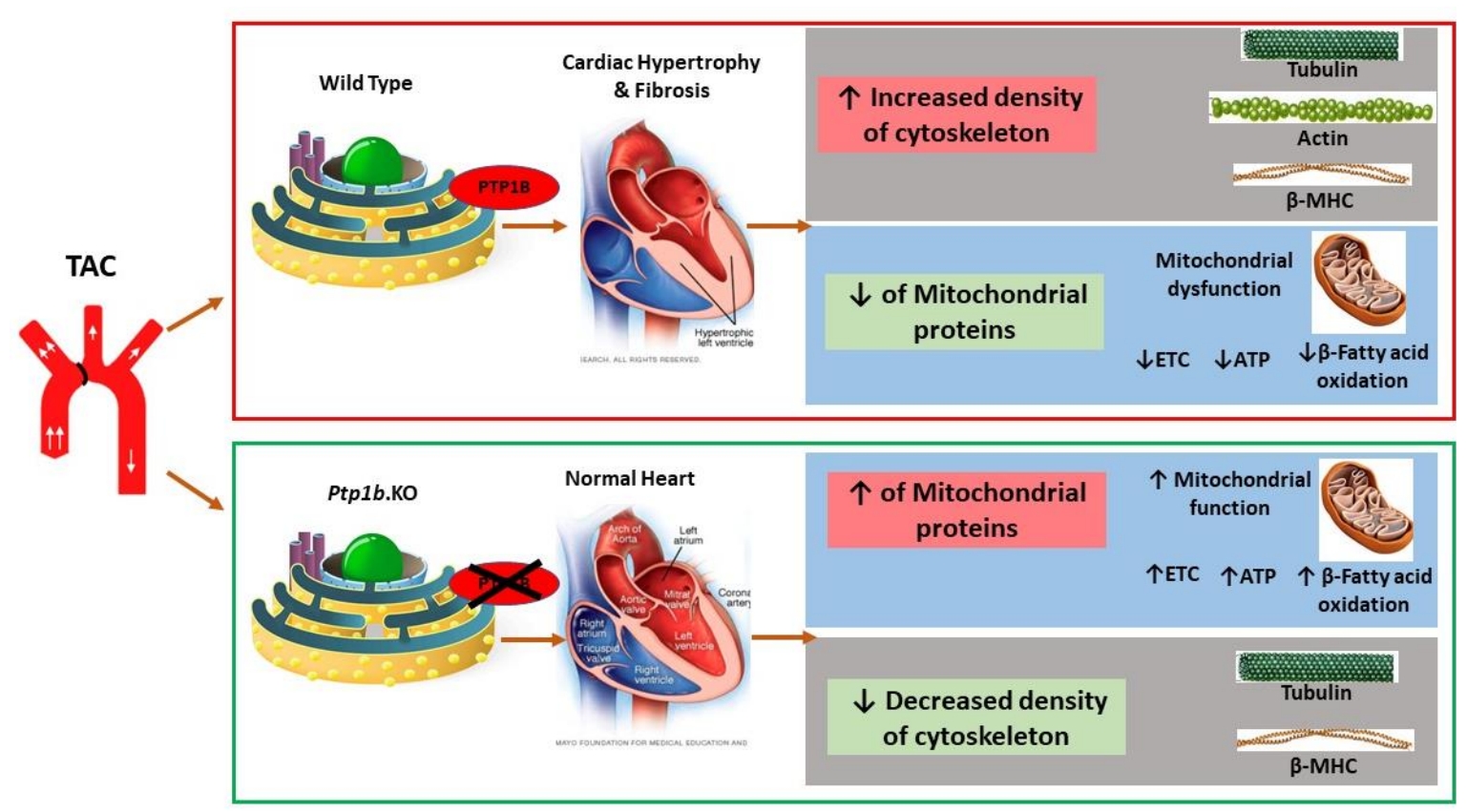

Figure 4.10 Summary of global proteome comparison in WT-TAC and KO-SHAM: TAC operation induces hypertrophy in wild type hearts (upper panel) due to up-regulation of cytoskeleton associated proteins and mitochondrial dysfunction due to down-regulation of mitochondrial proteins related to electron transport chain (ETC), ATP synthesis and Fatty acid metabolism. However, Ptp1b.KO mice hearts (lower panel) do not develop hypertrophy and fibrosis in response to TAC due to up-regulation of mitochondrial proteins and down-regulation of cytoskeleton associated proteins.

In summary our proteome data strongly supports that PTP1B deficiency protects the mice from cardiac hypertrophy and fibrosis due to improved mitochondrial function and reduced cytoskeleton density (Fig. 4.10). To support these observations, we performed in vitro studies using PTP1b inhibitor in ECs to confirm the improved mitochondrial function. We observed that TNF- $\alpha$ treatment induces the mitochondrial functional stress which leads to reduced production of ATP. This observation is supported a study by Kalogeris et al., 2014, who reported decrease in ATP production and mitochondrial mass in ECs in response to TNF-a (Kalogeris et al., 2014). Furthermore, we were able to analyse successfully that PTP1B inhibition prior to TNF- $\alpha$ treatment in ECs protects the cells from mitochondrial dysfunction as well as the restoration of ATP production.

In conclusion our study elaborates that BRD4 inhibition reduces the inflammation and could be a therapeutic target for atherosclerosis. Furthermore, PTP1B inhibition improves the cardiac function in pressure overloaded hearts by improving the mitochondrial function in Ptp1b-KO mice (Fig. 4.11). 

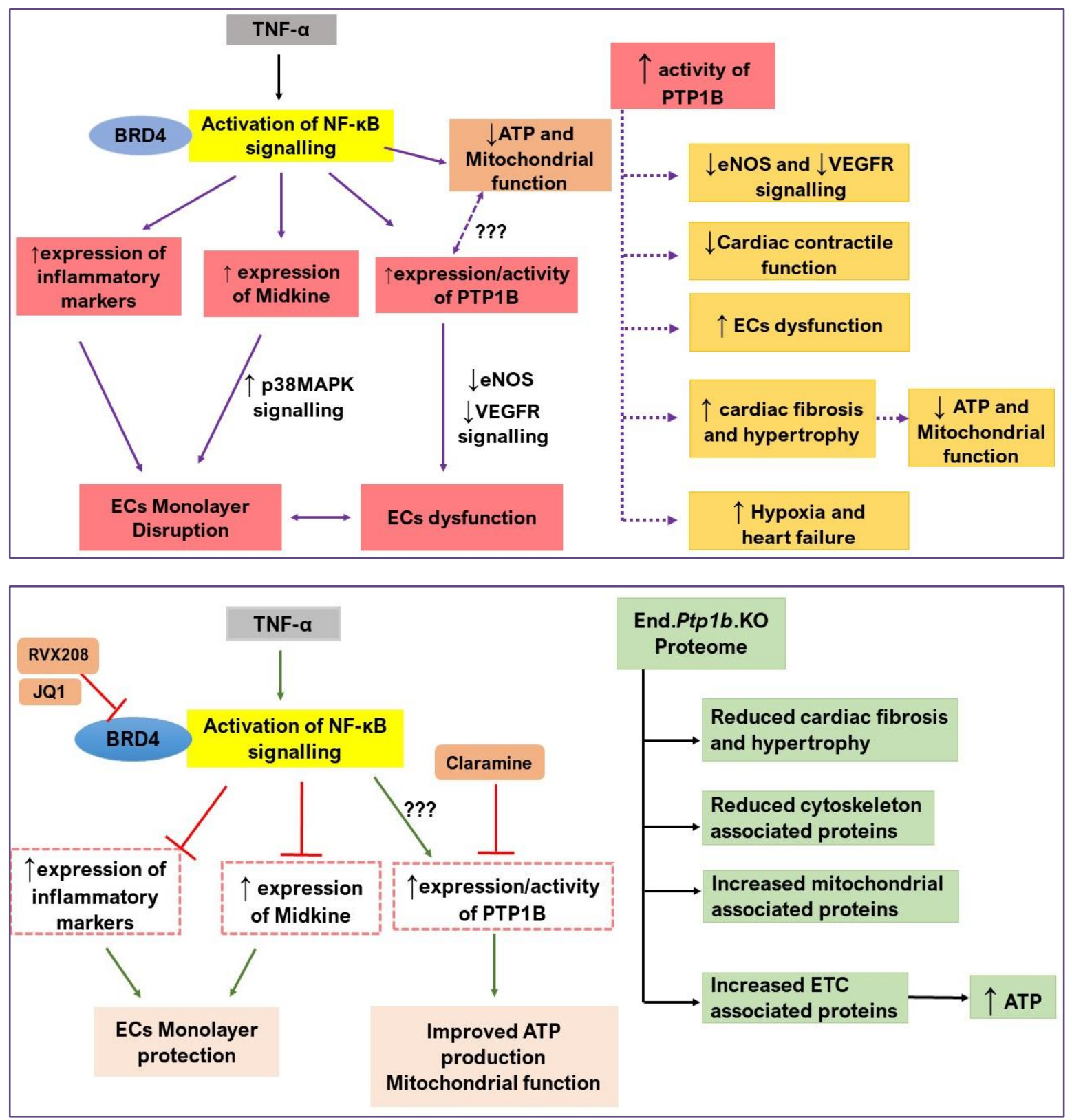

Figure 4.11 Conclusion: TNF- $\alpha$, BRD4 and PTP1B function on ECs and CVDs: Upper Panel :TNF- $\alpha$ induces activation of NF-KB, BRD4 and NF-KB transcribe inflammatory markers, Midkine and PTP1B. Increased expression of inflammatory markers and Midkine contributes in ECs dysfunction and monolayer disruption. Increased expression of PTP1B is known for reduced expression of eNOS and VEGFR signalling, an indication of ECs dysfunction. Increased activity of PTP1B have been reported to be associated with reduced cardiac contractile function, reduced ATP production, increased cardiac fibrosis, hypertrophy, ECs dysfunction, hypoxia and heart failure. Lower Panel: Our data showed RVX208/JQ1 based BRD4 inhibition reduces the expression of inflammatory markers, Midkine and it protects ECs monolayer from disruption. Further studies should be conducted to analyse the effect of BRD4 inhibition on PTP1B expression. Our data showed that PTP1B inhibition with Claramine recovers the ATP production loss induced by TNF- $\alpha$ treatment. Our data showed the Endo.Ptp1b.KO reduces the cardiac hypertrophy and fibrosis by reducing the cytoskeleton associated protein expression and increasing the mitochondrial protein expression to compensate the energy demand in pressure overloaded hearts. 


\section{Summary}

Vascular and lymphatic system is lined by Endothelial cells (ECs) monolayer or endothelium. Healthy endothelium maintains vessel functions by releasing different substances including Nitric Oxide (NO). Cardiovascular risk factors induce ECs dysfunction, which leads to the increase permeability of ECs and development of atherosclerosis. In response to inflammatory stimulus, NF-kB signalling pathway becomes activated. NF-KB signalling is responsible for releasing cytokines and expressing adhesion molecules on ECs surface. BRD4 is a chromatin regulator transcription factor, which binds to super enhancers of basal cell state maintaining gene loci. BRD4 redistributes on chromatin during inflammation and along with NF-KB binds to super enhancers of inflammatory gene loci.

In the present study, we analysed the role of BRD4 and its isoforms in ECs dysfunction and investigated whether BRD4 inhibition restores ECs function under inflammationstimulating conditions. We established an in vitro model of inflammatory phenotype using he treatment of HUVECs with TNF-a. Our study indicates that BRD4 short isoform is overexpressed in HUVECs during inflammation along with increased expression of inflammatory markers, Midkine, and PTP1B. Pre-treatment with BRD4 inhibitors (JQ1 and RVX-208) abrogates ECs activation and dysfunction via reducing the expression of pro inflammatory markers. We also observed that TNF- $\alpha$ treated HUVECs showed increased permeability which indicates disrupted monolayer integrity. In contrast, monolayer integrity was retained when HUVECs pre-treated with BRD4 inhibitors.

Elevated levels of Midkine has been reported in human and mice atherosclerotic plaques. It has also been reported to disrupt monolayer integrity via activation of downstream signalling pathways. HUVECs secrete Midkine when treated to 48-72 hours with TNF- $\alpha$. BRD4 inhibition with JQ1 and RVX208 reduced the Midkine secretion indicating BRD4 inhibition is retaining the monolayer integrity by reduce Midkine signalling. As far as we know, this is the first study that identified the role of BRD4 in regulation of Midkine, an upstream regulator of p38MAPK signalling pathway. Our data pointed out that an orally administered RVX208 drug can contribute in atherosclerosis treatment. Our study gives an indication that BRD4 inhibition can be used as a therapeutic option for treatment of atherosclerosis. 
Our results confirms the previous observations stating PTP1B inhibition is beneficial in ECs, in-vitro and in-vivo. This is the first study to report the heart global proteome of PTP1B-deficient mice and its contribution in prevention of cardiac fibrosis and hypertrophy. Our data shed light on cardio-protective role of PTP1B deletion by identifying the improvement of mitochondrial function in cardiac hypertrophy animals. Furthermore, this report proved in in-vitro study that PTP1B inhibition improves the ATP production in ECs. From our results, it can be suggested that PTP1B inhibition can be a therapeutic option in prevention of cardiac fibrosis and hypertrophy after myocardial infarction. This study provides essential understanding into beneficial roles of BRD4 and Ptp1b in CVDs, especially, atherosclerosis and cardiac hypertrophy. 


\section{Bibliography}

Aird, W. C. (2004) Endothelium as an organ system. Crit Care Med. 32:S271-S279.

Ali, M. I., P. Ketsawatsomkron, E. J. Belin de Chantemele, J. D. Mintz, K. Muta, C. Salet, S. M. Black, M. L. Tremblay, D. J. Fulton, M. B. Marrero, and D. W. Stepp. (2009) Deletion of protein tyrosine phosphatase $1 \mathrm{~b}$ improves peripheral insulin resistance and vascular function in obese, leptinresistant mice via reduced oxidant tone. Circ. Res. 105:1013-1022.

Alonso, A., J. Sasin, N. Bottini, I. Friedberg, I. Friedberg, A. Osterman, A. Godzik, T. Hunter, J. Dixon, and T. Mustelin. (2004) Protein tyrosine phosphatases in the human genome. Cell 117:699-711.

Alsarraj, J., F. Faraji, T. R. Geiger, K. R. Mattaini, M. Williams, J. Wu, N. H. Ha, T. Merlino, R. C. Walker, A. D. Bosley, Z. Xiao, T. Andresson, D. Esposito, N. Smithers, D. Lugo, R. Prinjha, A. Day, N. P. Crawford, K. Ozato, K. Gardner, and K. W. Hunter. (2013) BRD4 short isoform interacts with RRP1B, SIPA1 and components of the LINC complex at the inner face of the nuclear membrane. PLoS. One. 8:e80746.

Alsarraj, J., R. C. Walker, J. D. Webster, T. R. Geiger, N. P. Crawford, R. M. Simpson, K. Ozato, and K. W. Hunter. (2011) Deletion of the proline-rich region of the murine metastasis susceptibility gene Brd4 promotes epithelial-to-mesenchymal transition- and stem cell-like conversion. Cancer Res. 71:3121-3131.

Alvarez, B. V., A. L. Quon, J. Mullen, and J. R. Casey. (2013) Quantification of carbonic anhydrase gene expression in ventricle of hypertrophic and failing human heart. BMC. Cardiovasc. Disord. 13:2.

Ambler, C. A., J. L. Nowicki, A. C. Burke, and V. L. Bautch. (2001) Assembly of trunk and limb blood vessels involves extensive migration and vasculogenesis of somite-derived angioblasts. Dev. Biol. 234:352-364.

Anggard, E. E. (1990) The endothelium--the body's largest endocrine gland? J. Endocrinol. 127:371375.

Aquila-Pastir, L. A., N. R. DiPaola, R. G. Matteo, N. G. Smedira, P. M. McCarthy, and C. S. Moravec. (2002) Quantitation and distribution of beta-tubulin in human cardiac myocytes. J. Mol. Cell Cardiol. 34:1513-1523.

Arregui, C. O., A. Gonzalez, J. E. Burdisso, and A. E. Gonzalez Wusener. (2013) Protein tyrosine phosphatase PTP1B in cell adhesion and migration. Cell Adh. Migr. 7:418-423.

Aveleira, C. I. A., C. M. Lin, S. F. Abcouwer, A. F. Ambrã Ásio, and D. A. Antonetti. (2010) TNF-a Signals Through PKC/NF-kB to Alter the Tight Junction Complex and Increase Retinal Endothelial Cell Permeability. Diabetes. 59:2872-2882.

Banquet, S., E. Gomez, L. Nicol, F. Edwards-Levy, J. P. Henry, R. Cao, D. Schapman, B. Dautreaux, F. Lallemand, F. Bauer, Y. Cao, C. Thuillez, P. Mulder, V. Richard, and E. Brakenhielm. (2011) 
Arteriogenic therapy by intramyocardial sustained delivery of a novel growth factor combination prevents chronic heart failure. Circulation. 124:1059-1069.

Barr, A. J. (2010) Protein tyrosine phosphatases as drug targets: strategies and challenges of inhibitor development. Future. Med. Chem. 2:1563-1576.

Bence, K. K., M. Delibegovic, B. Xue, C. Z. Gorgun, G. S. Hotamisligil, B. G. Neel, and B. B. Kahn. (2006) Neuronal PTP1B regulates body weight, adiposity and leptin action. Nat. Med. 12:917-924.

Besnier, M., A. Galaup, L. Nicol, J. P. Henry, D. Coquerel, A. Gueret, P. Mulder, E. Brakenhielm, C. Thuillez, S. Germain, V. Richard, and A. Ouvrard-Pascaud. (2014) Enhanced angiogenesis and increased cardiac perfusion after myocardial infarction in protein tyrosine phosphatase 1Bdeficient mice. FASEB J. 28:3351-3361.

Bid, H. K. and S. Kerk. (2016) BET bromodomain inhibitor (JQ1) and tumor angiogenesis. Oncoscience. 3:316-317.

Bid, H. K., D. A. Phelps, L. Xaio, D. C. Guttridge, J. Lin, C. London, L. H. Baker, X. Mo, and P. J. Houghton. (2016) The Bromodomain BET Inhibitor JQ1 Suppresses Tumor Angiogenesis in Models of Childhood Sarcoma. Mol. Cancer Ther. 15:1018-1028.

Bleakley, C., P. K. Hamilton, R. Pumb, M. Harbinson, and G. E. McVeigh. (2015) Endothelial Function in Hypertension: Victim or Culprit? J. Clin Hypertens. 17:651-654.

Brown, J. D., C. Y. Lin, Q. Duan, G. Griffin, A. Federation, R. M. Paranal, S. Bair, G. Newton, A. Lichtman, A. Kung, T. Yang, H. Wang, F. W. Luscinskas, K. Croce, J. E. Bradner, and J. Plutzky. (2014) NF-kB directs dynamic super enhancer formation in inflammation and atherogenesis. Mol. Cell. 56:219-231.

Bruder-Nascimento, T., B. R. Butler, D. J. Herren, M. W. Brands, K. K. Bence, and E. J. Belin de Chantemele. (2015) Deletion of protein tyrosine phosphatase $1 \mathrm{~b}$ in proopiomelanocortin neurons reduces neurogenic control of blood pressure and protects mice from leptin- and sympathomediated hypertension. Pharmacol. Res. 102:235-244.

Bugger, H., M. Schwarzer, D. Chen, A. Schrepper, P. A. Amorim, M. Schoepe, T. D. Nguyen, F. W. Mohr, O. Khalimonchuk, B. C. Weimer, and T. Doenst. (2010) Proteomic remodelling of mitochondrial oxidative pathways in pressure overload-induced heart failure. Cardiovasc. Res. 85:376-384.

Callahan, M. K., K. A. Williams, P. Kivisakk, D. Pearce, M. F. Stins, and R. M. Ransohoff. (2004) CXCR3 marks CD4+ memory $\mathrm{T}$ lymphocytes that are competent to migrate across a human brain microvascular endothelial cell layer. J. Neuroimmunol. 153:150-157.

Carmeliet, P. (2005) Angiogenesis in life, disease and medicine. Nature 438:932-936.

Carreno, J. E., F. Apablaza, M. P. Ocaranza, and J. E. Jalil. (2006) Cardiac hypertrophy: molecular and cellular events. Rev. Esp. Cardiol. 59:473-486. 
Chaturvedi, N. 2003. ETHNIC DIFFERENCES IN CARDIOVASCULAR DISEASE. Heart. 89:681-686

Chen, W. Y., J. Hong, J. Gannon, R. Kakkar, and R. T. Lee. (2015) Myocardial pressure overload induces systemic inflammation through endothelial cell IL-33. Proc. Natl. Acad. Sci. U. S. A 112:7249-7254.

Cheng, S., V. R. Fernandes, D. A. Bluemke, R. L. McClelland, R. A. Kronmal, and J. A. Lima. (2009) Age-related left ventricular remodeling and associated risk for cardiovascular outcomes: the MultiEthnic Study of Atherosclerosis. Circ. Cardiovasc. Imaging 2:191-198.

Chiang, C. M. (2009) Brd4 engagement from chromatin targeting to transcriptional regulation: selective contact with acetylated histone $\mathrm{H} 3$ and H4. F100O. Biol. Rep. 1:98.

Chien, S. (2008) Effects of disturbed flow on endothelial cells. Ann. Biomed. Eng 36:554-562.

Chouchani, E. T., C. Methner, G. Buonincontri, C. H. Hu, A. Logan, S. J. Sawiak, M. P. Murphy, and T. Krieg. (2014) Complex I deficiency due to selective loss of Ndufs4 in the mouse heart results in severe hypertrophic cardiomyopathy. PLoS. One. 9:e94157.

Conti, P. and Y. Shaik-Dasthagirisaeb. (2015) Atherosclerosis: a chronic inflammatory disease mediated by mast cells. Cent. Eur. J. Immunol. 40:380-386.

Coquerel, D., R. Neviere, E. Delile, P. Mulder, X. Marechal, D. Montaigne, S. Renet, I. Remy-Jouet, E. Gomez, J. P. Henry, J. C. do Rego, V. Richard, and F. Tamion. (2014) Gene deletion of protein tyrosine phosphatase $1 \mathrm{~B}$ protects against sepsis-induced cardiovascular dysfunction and mortality. Arterioscler. Thromb. Vasc. Biol. 34:1032-1044.

Crawford, N. P., J. Alsarraj, L. Lukes, R. C. Walker, J. S. Officewala, H. H. Yang, M. P. Lee, K. Ozato, and K. W. Hunter. (2008) Bromodomain 4 activation predicts breast cancer survival. Proc. Natl. Acad. Sci. U. S. A 105:6380-6385.

Crowe, B. L., R. C. Larue, C. Yuan, S. Hess, M. Kvaratskhelia, and M. P. Foster. (2016) Structure of the Brd4 ET domain bound to a C-terminal motif from gamma-retroviral integrases reveals a conserved mechanism of interaction. Proc. Natl. Acad. Sci. U. S. A 113:2086-2091.

Dai, D. F., E. J. Hsieh, T. Chen, L. G. Menendez, N. B. Basisty, L. Tsai, R. P. Beyer, D. A. Crispin, N. J. Shulman, H. H. Szeto, R. Tian, M. J. MacCoss, and P. S. Rabinovitch. (2013) Global proteomics and pathway analysis of pressure-overload-induced heart failure and its attenuation by mitochondrialtargeted peptides. Circ. Heart Fail. 6:1067-1076.

Dai, D. F., E. J. Hsieh, Y. Liu, T. Chen, R. P. Beyer, M. T. Chin, M. J. MacCoss, and P. S. Rabinovitch. (2012) Mitochondrial proteome remodelling in pressure overload-induced heart failure: the role of mitochondrial oxidative stress. Cardiovasc. Res. 93:79-88.

Dart, A. M. and J. P. Chin-Dusting. (1999) Lipids and the endothelium. Cardiovasc. Res. 43:308-322.

Deaton, C., E. S. Froelicher, L. H. Wu, C. Ho, K. Shishani, and T. Jaarsma. (2011) The global burden of cardiovascular disease. Eur. J. Cardiovasc. Nurs. 10 Suppl 2:S5-13. 
Devaiah, B. N., C. Case-Borden, A. Gegonne, C. H. Hsu, Q. Chen, D. Meerzaman, A. Dey, K. Ozato, and D. S. Singer. (2016) BRD4 is a histone acetyltransferase that evicts nucleosomes from chromatin. Nat. Struct. Mol. Biol. 23:540-548.

Devaiah, B. N., B. A. Lewis, N. Cherman, M. C. Hewitt, B. K. Albrecht, P. G. Robey, K. Ozato, R. J. Sims, III, and D. S. Singer. (2012) BRD4 is an atypical kinase that phosphorylates serine2 of the RNA polymerase II carboxy-terminal domain. Proc. Natl. Acad. Sci. U. S. A 109:6927-6932.

Dhingra, R. and R. S. Vasan. (2011) Age as a Cardiovascular Risk Factor. med clin north america. Med Clin North Am. 96:87-91

Dickhout, J. G. and R. C. Austin. (2006) Proteasomal regulation of cardiac hypertrophy: is demolition necessary for building? Circulation 114:1796-1798.

Dormond, O., A. Foletti, C. Paroz, and C. Ruegg. (2001) NSAIDs inhibit alpha V beta 3 integrinmediated and $\mathrm{Cdc} 42 /$ Rac-dependent endothelial-cell spreading, migration and angiogenesis. Nat. Med. 7:1041-1047.

Drugs.R.D. (2011) Rvx 208. Drugs R. D. 11:207-213.

Elchebly, M., P. Payette, E. Michaliszyn, W. Cromlish, S. Collins, A. L. Loy, D. Normandin, A. Cheng, J. Himms-Hagen, C. C. Chan, C. Ramachandran, M. J. Gresser, M. L. Tremblay, and B. P. Kennedy. (1999) Increased insulin sensitivity and obesity resistance in mice lacking the protein tyrosine phosphatase-1B gene. Science 283:1544-1548.

Eshkiki, Z. S., M. H. Ghahremani, P. Shabani, S. G. Firuzjaee, A. Sadeghi, H. Ghanbarian, and R. Meshkani. (2017) Protein tyrosine phosphatase 1B (PTP1B) is required for cardiac lineage differentiation of mouse embryonic stem cells. Mol. Cell Biochem. 425:95-102.

Falk, E. (1992) Why do plaques rupture? Circulation 86:III30-III42.

Forouhi, N. G. and N. Sattar. (2006) CVD risk factors and ethnicity--a homogeneous relationship? Atheroscler. Suppl 7:11-19.

Friedl, J., M. Puhlmann, D. L. Bartlett, S. K. Libutti, E. N. Turner, M. F. Gnant, and H. R. Alexander. (2002) Induction of permeability across endothelial cell monolayers by tumor necrosis factor (TNF) occurs via a tissue factor-dependent mechanism: relationship between the procoagulant and permeability effects of TNF. Blood 100:1334-1339.

Geraghty, P., A. A. Hardigan, A. M. Wallace, O. Mirochnitchenko, J. Thankachen, L. Arellanos, V. Thompson, J. M. D'Armiento, and R. F. Foronjy. (2013) The glutathione peroxidase 1-protein tyrosine phosphatase $1 \mathrm{~B}$-protein phosphatase $2 \mathrm{~A}$ axis. A key determinant of airway inflammation and alveolar destruction. Am. J. Respir. Cell Mol. Biol. 49:721-730.

Gerhardt, T. and K. Ley. (2015) Monocyte trafficking across the vessel wall. Cardiovasc. Res. 107: 321-30 
Ghosh, G. C., R. Bhadra, R. K. Ghosh, K. Banerjee, and A. Gupta. (2017) RVX 208: A novel BET protein inhibitor, role as an inducer of apo A-I/HDL and beyond. Cardiovasc. Ther. 35. 1755.

Gilham, D., S. Wasiak, L. M. Tsujikawa, C. Halliday, K. Norek, R. G. Patel, E. Kulikowski, J. Johansson, M. Sweeney, and N. C. Wong. (2016) Corrigendum to "RVX-208, a BET-inhibitor for treating atherosclerotic cardiovascular disease, raises ApoA-I/HDL and represses pathways that contribute to cardiovascular disease". Atherosclerosis 253:345.

Gogiraju, R., M. R. Schroeter, M. L. Bochenek, A. Hubert, T. Munzel, G. Hasenfuss, and K. Schafer. (2016) Endothelial Deletion of Protein Tyrosine Phosphatase-1B Protects Against Pressure Overload-Induced Heart Failure in Mice. Cardiovasc. Res. 111: 204-16.

Gomez, E., M. Vercauteren, B. Kurtz, A. Ouvrard-Pascaud, P. Mulder, J. P. Henry, M. Besnier, A. Waget, H. R. Hooft Van, M. L. Tremblay, R. Burcelin, C. Thuillez, and V. Richard. (2012) Reduction of heart failure by pharmacological inhibition or gene deletion of protein tyrosine phosphatase $1 \mathrm{~B}$. J. Mol. Cell Cardiol. 52:1257-1264.

Gupta, M. P. (2007) Factors controlling cardiac myosin-isoform shift during hypertrophy and heart failure. J. Mol. Cell Cardiol. 43:388-403.

Hadi, H. A., C. S. Carr, and S. J. Al. (2005) Endothelial dysfunction: cardiovascular risk factors, therapy, and outcome. Vasc. Health Risk Manag. 1:183-198.

Hajra, L., A. I. Evans, M. Chen, S. J. Hyduk, T. Collins, and M. I. Cybulsky. (2000) The NF-kappa B signal transduction pathway in aortic endothelial cells is primed for activation in regions predisposed to atherosclerotic lesion formation. Proc. Natl. Acad. Sci. U. S. A 97:9052-9057.

Hara, H., N. Takeda, and I. Komuro. (2017) Pathophysiology and therapeutic potential of cardiac fibrosis. Inflamm. Regen. 37:13.

Heineke, J. and J. D. Molkentin. (2006) Regulation of cardiac hypertrophy by intracellular signalling pathways. Nat. Rev. Mol. Cell Biol. 7:589-600.

Herron, T. J., F. S. Korte, and K. S. McDonald. (2001) Loaded shortening and power output in cardiac myocytes are dependent on myosin heavy chain isoform expression. Am. J. Physiol Heart Circ. Physiol 281:H1217-H1222.

Higashi, Y., Y. Kihara, and K. Noma. (2012) Endothelial dysfunction and hypertension in aging. Hypertens. Res. 35:1039-1047.

Holderfield, M. T. and C. C. Hughes. (2008) Crosstalk between vascular endothelial growth factor, notch, and transforming growth factor-beta in vascular morphogenesis. Circ. Res. 102:637-652.

Hu, Y., J. Zhou, F. Ye, H. Xiong, L. Peng, Z. Zheng, F. Xu, M. Cui, C. Wei, X. Wang, Z. Wang, H. Zhu, P. Lee, M. Zhou, B. Jiang, and D. Y. Zhang. (2015) BRD4 inhibitor inhibits colorectal cancer growth and metastasis. Int. J. Mol. Sci. 16:1928-1948. 
Huang, B., X. D. Yang, M. M. Zhou, K. Ozato, and L. F. Chen. (2009) Brd4 coactivates transcriptional activation of NF-kappaB via specific binding to acetylated RelA. Mol. Cell Biol. 29:1375-1387.

Huang, M., Q. Qiu, Y. Xiao, S. Zeng, M. Zhan, M. Shi, Y. Zou, Y. Ye, L. Liang, X. Yang, and H. Xu. (2016) BET Bromodomain Suppression Inhibits VEGF-induced Angiogenesis and Vascular Permeability by Blocking VEGFR2-mediated Activation of PAK1 and eNOS. Sci. Rep. 6:23770.

Huang, M., S. Zeng, Y. Zou, M. Shi, Q. Qiu, Y. Xiao, G. Chen, X. Yang, L. Liang, and H. Xu. (2017) The suppression of bromodomain and extra-terminal domain inhibits vascular inflammation by blocking NF-kappaB and MAPK activation. Br. J. Pharmacol. 174:101-115.

Hudson, B., C. Hidalgo, C. Saripalli, and H. Granzier. (2011) Hyperphosphorylation of mouse cardiac titin contributes to transverse aortic constriction-induced diastolic dysfunction. Circ. Res. 109:858866.

Hueso, L., C. Rios-Navarro, A. Ruiz-Sauri, F. J. Chorro, J. Nunez, M. J. Sanz, V. Bodi, and L. Piqueras. (2017) Dynamics and implications of circulating anti-angiogenic VEGF-A165b isoform in patients with ST-elevation myocardial infarction. Sci. Rep. 7:9962.

Huq, Michiko Moriyama, and Pradeep Kumar Ray. (2018) Risk Factors for Development of Cardiovascular Disease. Asian Hosptal and Health care management report https://www.asianhhm.com/medical-sciences/risk-factors-development-cardiovascular-disease.

Imes, C. C. and F. M. Lewis. (2014) Family history of cardiovascular disease (CVD), perceived CVD risk, and health-related behavior: A review of the literature. J. Cardiovasc. Nurs. 29:108-29.

Isogai, C., W. E. Laug, H. Shimada, P. J. Declerck, M. F. Stins, D. L. Durden, A. Erdreich-Epstein, and Y. A. DeClerck. (2001) Plasminogen activator inhibitor-1 promotes angiogenesis by stimulating endothelial cell migration toward fibronectin. Cancer Res. 61:5587-5594.

Jeon, Y. M., S. Lee, S. Kim, Y. Kwon, K. Kim, C. G. Chung, S. Lee, S. B. Lee, and H. J. Kim. (2017) Neuroprotective Effects of Protein Tyrosine Phosphatase 1B Inhibition against ER Stress-Induced Toxicity. Mol. Cells 40:280-290.

Ji, K. T., L. Qian, J. L. Nan, Y. J. Xue, S. Q. Zhang, G. Q. Wang, R. P. Yin, Y. J. Zhu, L. P. Wang, J. Ma, L. M. Liao, and J. F. Tang. (2015) Ox-LDL induces dysfunction of endothelial progenitor cells via activation of NF-kappaB. Biomed. Res. Int. 2015:175291.

Jiao, H., Y. Zhang, Z. Yan, Z. G. Wang, G. Liu, R. D. Minshall, A. B. Malik, and G. Hu. (2013) Caveolin1 Tyr14 phosphorylation induces interaction with TLR4 in endothelial cells and mediates MyD88dependent signaling and sepsis-induced lung inflammation. J. Immunol. 191:6191-6199.

Jing, L., C. M. Binkley, J. D. Suever, N. Umasankar, C. M. Haggerty, J. Rich, G. J. Wehner, S. M. Hamlet, D. K. Powell, A. Radulescu, H. L. Kirchner, F. H. Epstein, and B. K. Fornwalt. (2016) Cardiac remodeling and dysfunction in childhood obesity: a cardiovascular magnetic resonance study. J. Cardiovasc. Magn Reson. 18:28. 
Kachur, S., C. J. Lavie, S. A. de, R. V. Milani, and H. O. Ventura. (2017) Obesity and cardiovascular diseases. Minerva Med. 108:212-228.

Kadomatsu, K., P. Bencsik, A. Gorbe, C. Csonka, K. Sakamoto, S. Kishida, and P. Ferdinandy. (2014) Therapeutic potential of midkine in cardiovascular disease. Br. J. Pharmacol. 171:936-944.

Kalogeris, T. J., C. Baines, and R. J. Korthuis. (2014) Adenosine prevents TNFalpha-induced decrease in endothelial mitochondrial mass via activation of eNOS-PGC-1alpha regulatory axis. PLOS. One. 9:e98459.

Kathiresan, S. and D. Srivastava. (2012) Genetics of Human Cardiovascular Disease. Cell. 148:124257.

Kazakoff, P. W., T. R. McGuire, E. B. Hoie, M. Cano, and P. L. Iversen. (1995) An in vitro model for endothelial permeability: assessment of monolayer integrity. In Vitro Cell Dev. Biol. Anim. 31:846852.

Kempen, H. J., D. Bellus, O. Fedorov, S. Nicklisch, P. Filippakopoulos, S. Picaud, and S. Knapp. (2013) Stimulation of Hepatic Apolipoprotein A-I Production by Novel Thieno-Triazolodiazepines: Roles of the Classical Benzodiazepine Receptor, PAF Receptor, and Bromodomain Binding. Lipid Insights. 6:47-54.

Khan, N., L. Binder, D. V. K. Pantakani, and A. R. Asif. (2017) MPA Modulates Tight Junctions' Permeability via Midkine/PI3K Pathway in Caco-2 Cells: A Possible Mechanism of Leak-Flux Diarrhea in Organ Transplanted Patients. Front Physiol 8:438.

Khan, N., C. Lenz, L. Binder, D. V. Pantakani, and A. R. Asif. (2016) Active and Repressive ChromatinAssociated Proteome after MPA Treatment and the Role of Midkine in Epithelial Monolayer Permeability. Int. J. Mol. Sci. 17: E597

Kim, J., M. Montagnani, S. Chandrasekran, and M. J. Quon. (2012) Role of Lipotoxicity in Endothelial Dysfunction. Heart Fail. Clin. 8: 589-607.

Kobayashi, K., K. Maeda, M. Takefuji, R. Kikuchi, Y. Morishita, M. Hirashima, and T. Murohara. (2017) Dynamics of angiogenesis in ischemic areas of the infarcted heart. Sci. Rep. 7:7156.

Kolber, M. R. and C. Scrimshaw. (2014) Family history of cardiovascular disease. Can. Fam. Physcian. 60(11): 1016.

Kritchevsky, S. B., M. Cesari, and M. Pahor. (2005) Inflammatory markers and cardiovascular health in older adults. Cardiovasc. Res. 66:265-275.

Kurtenbach, S., S. Kurtenbach, and G. Zoidl. (2014) Gap junction modulation and its implications for heart function. Front Physiol 5:82.

Kwak, B. R., M. Back, M. L. Bochaton-Piallat, G. Caligiuri, M. J. Daemen, P. F. Davies, I. E. Hoefer, P. Holvoet, H. Jo, R. Krams, S. Lehoux, C. Monaco, S. Steffens, R. Virmani, C. Weber, J. J. Wentzel, and 
P. C. Evans. (2014) Biomechanical factors in atherosclerosis: mechanisms and clinical implications. Eur. Heart J. 35:3013-3020d.

Lamalice, L., B. F. Le, and J. Huot. (2007) Endothelial cell migration during angiogenesis. Circ. Res. 100:782-794.

Lanahan, A. A., D. Lech, A. Dubrac, J. Zhang, Z. W. Zhuang, A. Eichmann, and M. Simons. (2014) PTP1b is a physiologic regulator of vascular endothelial growth factor signaling in endothelial cells. Circulation 130:902-909.

Lau, E., Q. Cao, M. P. Y. Lam, J. Wang, D. C. M. Ng, B. J. Bleakley, J. M. Lee, D. A. Liem, D. Wang, H. Hermjakob, and P. Ping. (2018) Integrated omics dissection of proteome dynamics during cardiac remodeling. Nat. Commun. 9:120.

Lee, D., A. Kraus, D. Prins, J. Groenendyk, I. Aubry, W. X. Liu, H. D. Li, O. Julien, N. Touret, B. D. Sykes, M. L. Tremblay, and M. Michalak. (2015) UBC9-dependent association between calnexin and protein tyrosine phosphatase 1B (PTP1B) at the endoplasmic reticulum. J. Biol. Chem. 290:5725-5738.

Leon, B. M. and T. M. Maddox. (2015) Diabetes and cardiovascular disease: Epidemiology, biological mechanisms, treatment recommendations and future research. World Journal Diabetes. 13: $1246-58$

Leon, I. R., V. Schwammle, O. N. Jensen, and R. R. Sprenger. (2013) Quantitative assessment of insolution digestion efficiency identifies optimal protocols for unbiased protein analysis. Mol. Cell Proteomics. 12:2992-3005.

Libby, P. (2000) Changing concepts of atherogenesis. J. Intern. Med. 247:349-358.

Lovren, F., H. Teoh, and S. Verma. (2015) Obesity and atherosclerosis: mechanistic insights. Can. J. Cardiol. 31:177-183.

Low, J. L., C. L. Chai, and S. Q. Yao. (2014) Bidentate inhibitors of protein tyrosine phosphatases. Antioxid. Redox. Signal. 20:2225-2250.

Lu, H., P. W. Fedak, X. Dai, C. Du, Y. Q. Zhou, M. Henkelman, P. S. Mongroo, A. Lau, H. Yamabi, A. Hinek, M. Husain, G. Hannigan, and J. G. Coles. (2006) Integrin-linked kinase expression is elevated in human cardiac hypertrophy and induces hypertrophy in transgenic mice. Circulation 114:22712279.

Lu, P., Y. Shen, H. Yang, Y. Wang, Z. Jiang, X. Yang, Y. Zhong, H. Pan, J. Xu, H. Lu, and H. Zhu. (2017) BET inhibitors RVX-208 and PFI-1 reactivate HIV-1 from latency. Sci. Rep. 7:16646.

Mahdavi, V., A. P. Chambers, and B. Nadal-Ginard. (1984) Cardiac alpha- and beta-myosin heavy chain genes are organized in tandem. Proc. Natl. Acad. Sci. U. S. A 81:2626-2630.

Man, S., E. E. Ubogu, K. A. Williams, B. Tucky, M. K. Callahan, and R. M. Ransohoff. (2008) Human brain microvascular endothelial cells and umbilical vein endothelial cells differentially facilitate 
leukocyte recruitment and utilize chemokines for $\mathrm{T}$ cell migration. Clin Dev. Immunol. 2008:384982.

Manabe, I., T. Shindo, and R. Nagai. (2002) Gene expression in fibroblasts and fibrosis: involvement in cardiac hypertrophy. Circ. Res. 91:1103-1113.

Manning, G., D. B. Whyte, R. Martinez, T. Hunter, and S. Sudarsanam. (2002) The protein kinase complement of the human genome. Science 298:1912-1934.

Matz, R. L. and R. Andriantsitohaina. (2003) Age-related endothelial dysfunction : potential implications for pharmacotherapy. Drugs Aging 20:527-550.

McGrath, M. J., D. L. Cottle, M. A. Nguyen, J. M. Dyson, I. D. Coghill, P. A. Robinson, M. Holdsworth, B. S. Cowling, E. C. Hardeman, C. A. Mitchell, and S. Brown. (2006) Four and a half LIM protein 1 binds myosin-binding protein $\mathrm{C}$ and regulates myosin filament formation and sarcomere assembly. J. Biol. Chem. 281:7666-7683.

McLure, K. G., E. M. Gesner, L. Tsujikawa, O. A. Kharenko, S. Attwell, E. Campeau, S. Wasiak, A. Stein, A. White, E. Fontano, R. K. Suto, N. C. Wong, G. S. Wagner, H. C. Hansen, and P. R. Young. (2013) RVX-208, an inducer of ApoA-I in humans, is a BET bromodomain antagonist. PLoS. One. 8:e83190.

Messner, B. and D. Bernhard. (2014) Smoking and cardiovascular disease: mechanisms of endothelial dysfunction and early atherogenesis. Arterioscler. Thromb. Vasc. Biol. 34:509-515.

Michael Pittilo, R. (2000) Cigarette smoking, endothelial injury and cardiovascular disease. int. J. Exp. Pathol. 4:219-230

Michaelis, U. R. (2014) Mechanisms of endothelial cell migration. Cell Mol. Life Sci. 71:4131-4148.

Mobasher, M. A., A. Gonzalez-Rodriguez, B. Santamaria, S. Ramos, M. A. Martin, L. Goya, P. Rada, L. Letzig, L. P. James, A. Cuadrado, J. Martin-Perez, K. J. Simpson, J. Muntane, and A. M. Valverde. (2013) Protein tyrosine phosphatase 1B modulates GSK3beta/Nrf2 and IGFIR signaling pathways in acetaminophen-induced hepatotoxicity. Cell Death. Dis. 4:e626.

Moens, A. L., E. Takimoto, C. G. Tocchetti, K. Chakir, D. Bedja, G. Cormaci, E. A. Ketner, M. Majmudar, K. Gabrielson, M. K. Halushka, J. B. Mitchell, S. Biswal, K. M. Channon, M. S. Wolin, N. J. Alp, N. Paolocci, H. C. Champion, and D. A. Kass. (2008) Reversal of cardiac hypertrophy and fibrosis from pressure overload by tetrahydrobiopterin: efficacy of recoupling nitric oxide synthase as a therapeutic strategy. Circulation. 117:2626-2636.

Monaco, C. and E. Paleolog. (2004) Nuclear factor kappaB: a potential therapeutic target in atherosclerosis and thrombosis. Cardiovasc. Res. 61:671-682.

Montecucco, F., V. Braunersreuther, G. L. Viviani, S. Ã. Lenglet, and F. o. Mach. (2012) Update on the Pathophysiological Role of Intracellular Signaling Pathways in Atherosclerotic Plaques and Ischemic Myocardium. Current Signal Transduction Therapy. 2:104-110. 
Mottonen, M. J., O. Ukkola, J. Lumme, Y. A. Kesaniemi, H. V. Huikuri, and J. S. Perkiomaki. (2017) Cardiac Remodeling from Middle Age to Senescence. Front Physiol 8:341.

Najafova, Z., R. Tirado-Magallanes, M. Subramaniam, T. Hossan, G. Schmidt, S. Nagarajan, S. J. Baumgart, V. K. Mishra, U. Bedi, E. Hesse, S. Knapp, J. R. Hawse, and S. A. Johnsen. (2017) BRD4 localization to lineage-specific enhancers is associated with a distinct transcription factor repertoire. Nucleic Acids Res. 45:127-141.

Nakamura, Y., N. Patrushev, H. Inomata, D. Mehta, N. Urao, H. W. Kim, M. Razvi, V. Kini, K. Mahadev, B. J. Goldstein, R. McKinney, T. Fukai, and M. Ushio-Fukai. (2008) Role of protein tyrosine phosphatase $1 \mathrm{~B}$ in vascular endothelial growth factor signaling and cell-cell adhesions in endothelial cells. Circ. Res. 102:1182-1191.

Narishige, T., K. L. Blade, Y. Ishibashi, T. Nagai, M. Hamawaki, D. R. Menick, D. Kuppuswamy, and G. Cooper. (1999) Cardiac hypertrophic and developmental regulation of the beta-tubulin multigene family. J. Biol. Chem. 274:9692-9697.

Nicoletti, A. and J. B. Michel. (1999) Cardiac fibrosis and inflammation: interaction with hemodynamic and hormonal factors. Cardiovasc. Res. 41:532-543.

Nikolic, D., M. Rizzo, D. P. Mikhailidis, N. C. Wong, and M. Banach. (2015) An evaluation of RVX208 for the treatment of atherosclerosis. Expert. Opin. Investig. Drugs 24:1389-1398.

Panzhinskiy, E., J. Ren, and S. Nair. (2013) Protein tyrosine phosphatase 1B and insulin resistance: role of endoplasmic reticulum stress/reactive oxygen species/nuclear factor kappa B axis. PLoS. One. 8:e77228.

Picaud, S., C. Wells, I. Felletar, D. Brotherton, S. Martin, P. Savitsky, B. Diez-Dacal, M. Philpott, C. Bountra, H. Lingard, O. Fedorov, S. MÃ¹/4ller, P. E. Brennan, S. Knapp, and P. Filippakopoulos. (2013) RVX-208, an inhibitor of BET transcriptional regulators with selectivity for the second bromodomain. Proc. Natl. Acad. Sci. U. S. A. 49: 19754-9

Ramji, D. P. and T. S. Davies. (2015) Cytokines in atherosclerosis: Key players in all stages of disease and promising therapeutic targets. Cytokine Growth Factor Rev. 26:673-685.

Rio, D. C., M. Ares, Jr., G. J. Hannon, and T. W. Nilsen. (2010) Purification of RNA using TRIzol (TRI reagent). Cold Spring Harb. Protoc. 6:prot5439.

Rosca, M. G. and C. L. Hoppel. (2013) Mitochondrial dysfunction in heart failure. Heart Fail. Rev. 18:607-622.

Rosca, M. G., B. Tandler, and C. L. Hoppel. (2013) Mitochondria in cardiac hypertrophy and heart failure. J. Mol. Cell Cardiol. 55:31-41.

Rosiek, A. and K. Leksowski. (2016) The risk factors and prevention of cardiovascular disease: the importance of electrocardiogram in the diagnosis and treatment of acute coronary syndrome. Ther. Clin. Risk Manag. 12:1223-1229. 
Royall, J. A., R. L. Berkow, J. S. Beckman, M. K. Cunningham, S. Matalon, and B. A. Freeman. (1989) Tumor necrosis factor and interleukin 1 alpha increase vascular endothelial permeability. Am. J. Physiol 257:L399-L410.

Salaru, D., C. Arsenescu-Georgescu, Z. Halloul, M. Damerau, C. Albert, and P. R. Mertens. (2013) Expression of midkine, a multifunctional cytokine, in human atherosclerotic plaques. European Journal of Internal Medicine. 24: e38.

Salaru, D. L., C. Arsenescu-Georgescu, C. Chatzikyrkou, J. Karagiannis, A. Fischer, and P. R. Mertens. (2016) Midkine, a heparin-binding growth factor, and its roles in atherogenesis and inflammatory kidney diseases. Nephrol. Dial. Transplant. 31:1781-1787.

Samak, M., J. Fatullayev, A. Sabashnikov, M. Zeriouh, B. Schmack, M. Farag, A. F. Popov, P. M. Dohmen, Y. H. Choi, T. Wahlers, and A. Weymann. (2016) Cardiac Hypertrophy: An Introduction to Molecular and Cellular Basis. Med Sci. Monit. Basic Res. 22:75-79.

Schmittgen, T. D. and K. J. Livak. (2008) Analyzing real-time PCR data by the comparative C(T) method. Nat. Protoc. 3:1101-1108.

Sessa, W. C. (2004) eNOS at a glance. J. Cell Sci. 117:2427-2429.

Shimizu, I. and T. Minamino. (2016) Physiological and pathological cardiac hypertrophy. J. Mol. Cell Cardiol. 97:245-262.

Souders, C. A., T. K. Borg, I. Banerjee, and T. A. Baudino. (2012) Pressure overload induces early morphological changes in the heart. Am. J. Pathol. 181:1226-1235.

Srinivasan, B., A. R. Kolli, M. B. Esch, H. E. Abaci, M. L. Shuler, and J. J. Hickman. (2015) TEER measurement techniques for in vitro barrier model systems. J. Lab Autom. 20:107-126.

Stewart, J., G. Manmathan, and P. Wilkinson. (2017) Primary prevention of cardiovascular disease: A review of contemporary guidance and literature. JRSM. Cardiovasc. Dis. 6:2048004016687211.

Strait, J. B. and E. G. Lakatta. (2012) Aging-associated cardiovascular changes and their relationship to heart failure. Heart Fail. Clin. 8:143-164.

Stratton, M. S., S. M. Haldar, and T. A. McKinsey. (2017) BRD4 inhibition for the treatment of pathological organ fibrosis. F1000Res. 6:1015

Sumi, T., K. Matsumoto, Y. Takai, and T. Nakamura. (1999) Cofilin phosphorylation and actin cytoskeletal dynamics regulated by rho- and Cdc42-activated LIM-kinase 2. J. Cell Biol. 147:15191532.

Sumi-Ichinose, C., Y. Suganuma, T. Kano, N. Ihira, H. Nomura, K. Ikemoto, T. Hata, S. Katoh, H. Ichinose, and K. Kondo. (2017) Sepiapterin reductase gene-disrupted mice suffer from hypertension with fluctuation and bradycardia. Physiol Rep. 6: e13196 
Tagawa, H., N. Wang, T. Narishige, D. E. Ingber, M. R. Zile, and G. Cooper. (1997) Cytoskeletal mechanics in pressure-overload cardiac hypertrophy. Circ. Res. 80:281-289.

Tak, P. P. and G. S. Firestein. (2001) NF-kappaB: a key role in inflammatory diseases. J. Clin. Invest 107:7-11.

Talman, V. and H. Ruskoaho. (2016) Cardiac fibrosis in myocardial infarction-from repair and remodeling to regeneration. Cell Tissue Res. 365:563-581.

Tamrakar, A. K., C. K. Maurya, and A. K. Rai. (2014) PTP1B inhibitors for type 2 diabetes treatment: a patent review. Expert. Opin. Ther. Pat 24:1101-1115.

Tanaka, T. and M. lino. (2016) Nuclear Translocation of p65 is Controlled by Sec6 via the Degradation of IkappaBalpha. J. Cell Physiol 231:719-730.

Tardiff, J. C. (2006) Cardiac hypertrophy: stressing out the heart. J. Clin Invest 116:1467-1470.

Thiebaut, P. A., M. Besnier, E. Gomez, and V. Richard. (2016) Role of protein tyrosine phosphatase $1 \mathrm{~B}$ in cardiovascular diseases. J. Mol. Cell Cardiol. 101:50-57.

Thompson, D., N. Morrice, L. Grant, S. S. Le, K. Ziegler, P. Whitfield, N. Mody, H. M. Wilson, and M. Delibegovic. (2017) Myeloid protein tyrosine phosphatase 1B (PTP1B) deficiency protects against atherosclerotic plaque formation in the $\mathrm{ApoE}(-/-)$ mouse model of atherosclerosis with alterations in IL10/AMPKalpha pathway. Mol. Metab 6:845-853.

Tian, J., X. An, and L. Niu. (2017) Myocardial fibrosis in congenital and pediatric heart disease. Exp. Ther. Med 13:1660-1664.

Toischer, K., A. G. Rokita, B. Unsold, W. Zhu, G. Kararigas, S. Sossalla, S. P. Reuter, A. Becker, N. Teucher, T. Seidler, C. Grebe, L. Preuss, S. N. Gupta, K. Schmidt, S. E. Lehnart, M. Kruger, W. A. Linke, J. Backs, V. Regitz-Zagrosek, K. Schafer, L. J. Field, L. S. Maier, and G. Hasenfuss. (2010) Differential cardiac remodeling in preload versus afterload. Circulation 122:993-1003.

Tonks, N. K., C. D. Diltz, and E. H. Fischer. (1988) Purification of the major protein-tyrosinephosphatases of human placenta. J. Biol. Chem. 263:6722-6730.

Travers, J. G., F. A. Kamal, J. Robbins, K. E. Yutzey, and B. C. Blaxall. (2016) Cardiac Fibrosis: The Fibroblast Awakens. Circ. Res. 118:1021-1040.

Vasquez-Trincado, C., I. Garcia-Carvajal, C. Pennanen, V. Parra, J. A. Hill, B. A. Rothermel, and S. Lavandero. (2016) Mitochondrial dynamics, mitophagy and cardiovascular disease. J. Physiol 594:509-525.

Verstraete, M. (1990) Coronary atherosclerosis and thrombosis. Recenti Prog. Med 81:221-227.

Vidler, L. R., P. Filippakopoulos, O. Fedorov, S. Picaud, S. Martin, M. Tomsett, H. Woodward, N. Brown, S. Knapp, and S. Hoelder. (2013) Discovery of novel small-molecule inhibitors of BRD4 using structure-based virtual screening. J. Med. Chem. 56:8073-8088. 
von der Thusen, J. H., J. Kuiper, T. J. van Berkel, and E. A. Biessen. (2003) Interleukins in atherosclerosis: molecular pathways and therapeutic potential. Pharmacol. Rev. 55:133-166.

Voorhees, R. M., I. S. Fernandez, S. H. Scheres, and R. S. Hegde. (2014) Structure of the mammalian ribosome-Sec61 complex to 3.4 A resolution. Cell 157:1632-1643.

Wadhwa, E. and T. Nicolaides. (2016) Bromodomain Inhibitor Review: Bromodomain and Extraterminal Family Protein Inhibitors as a Potential New Therapy in Central Nervous System Tumors. cureus. 8(5):e620

Wang, L., X. Wu, P. Huang, Z. Lv, Y. Qi, X. Wei, P. Yang, and F. Zhang. (2016) JQ1, a small molecule inhibitor of BRD4, suppresses cell growth and invasion in oral squamous cell carcinoma. Oncol. Rep. 36:1989-1996.

Wang, Y., F. Yan, W. Zhang, S. Pang, and F. Jiang. (2018) Inhibiting Protein Tyrosine Phosphatase $1 \mathrm{~B}$ to Improve Regenerative Functions of Endothelial Cells. J. Cardiovasc. Pharmacol. 71:59-64.

Wang, Z. and T. Nakayama. (2010) Inflammation, a link between obesity and cardiovascular disease. Mediators. Inflamm. 2010:535918.

Wasiak, S., D. Gilham, L. M. Tsujikawa, C. Halliday, K. Norek, R. G. Patel, K. G. McLure, P. R. Young, A. Gordon, E. Kulikowski, J. Johansson, M. Sweeney, and N. C. Wong. (2016) Data on gene and protein expression changes induced by apabetalone (RVX-208) in ex vivo treated human whole blood and primary hepatocytes. Data Brief. 8:1280-1288.

Wende, A. R., J. Kim, W. L. Holland, B. E. Wayment, B. T. O'Neill, J. Tuinei, M. K. Brahma, M. E. Pepin, M. A. McCrory, I. Luptak, G. V. Halade, S. E. Litwin, and E. D. Abel. (2017) Glucose transporter 4-deficient hearts develop maladaptive hypertrophy in response to physiological or pathological stresses. Am. J. Physiol Heart Circ. Physiol 313:H1098-H1108.

Wong, F., C. Hull, R. Zhande, J. Law, and A. Karsan. (2004) Lipopolysaccharide initiates a TRAF6mediated endothelial survival signal. Blood 103:4520-4526.

Xiao, L., Y. Liu, and N. Wang. (2014) New paradigms in inflammatory signaling in vascular endothelial cells. Am. J. Physiol Heart Circ. Physiol 306:H317-H325.

Xu, Y. and C. R. Vakoc. (2014) Brd4 is on the move during inflammation. Trends cell biol. 24:615616.

Yip, S. C., S. Saha, and J. Chernoff. (2010) PTP1B: a double agent in metabolism and oncogenesis. Trends Biochem. Sci. 35:442-449.

Zabolotny, J. M., Y. B. Kim, L. A. Welsh, E. E. Kershaw, B. G. Neel, and B. B. Kahn. (2008) Proteintyrosine phosphatase $1 B$ expression is induced by inflammation in vivo. J. Biol. Chem. 283:1423014241. 
Zampetaki, A., L. Zeng, A. Margariti, Q. Xiao, H. Li, Z. Zhang, A. E. Pepe, G. Wang, O. Habi, E. deFalco, G. Cockerill, J. C. Mason, Y. Hu, and Q. Xu. (2010) Histone deacetylase 3 is critical in endothelial survival and atherosclerosis development in response to disturbed flow. Circulation 121:132-142.

Zengerle, M., K. H. Chan, and A. Ciulli. (2015) Selective Small Molecule Induced Degradation of the BET Bromodomain Protein BRD4. ACS Chem. Biol. 10:1770-1777.

Zhang, H., Y. Park, J. Wu, X. Chen, S. Lee, J. Yang, K. C. Dellsperger, and C. Zhang. (2009) Role of TNF-alpha in vascular dysfunction. Clin Sci. 116:219-230. 


\section{Supplementary material}

\section{Supplementary figure. S1: STRING cluster analysis}

A: WT-SHAM vs KO-SHAM up-regulated protein cluster B: WT-SHAM vs KO-SHAM down-regulated protein cluster

A
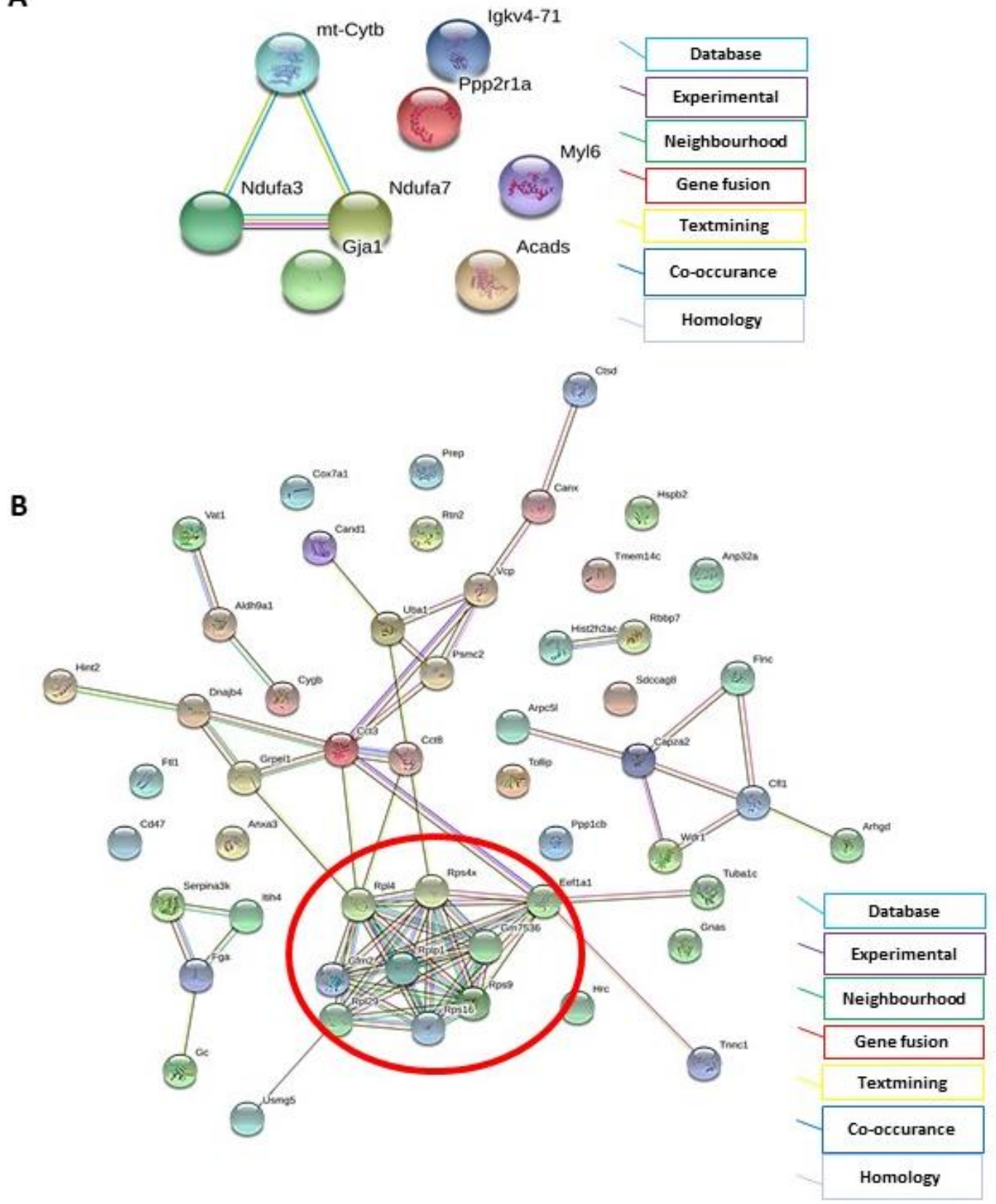

Fig. S1. Full view of STRING analysis of Group A, WT-SHAM vs KO-SHAM proteome. Analysis of protein interaction network by STRING, Coloured lines between the proteins indicate the protein co-occurrence, coexpression, known interactions and predicted interactions. Up-regulated proteins do not present any specific interaction or cluster (A) however Interconnecting ribosomal proteins (red circle) represents a functionally associated protein cluster in down-regulated proteins (B). 


\section{Supplementary figure. S2: STRING cluster analysis}

WT-SHAM vs WT-TAC up-regulated protein cluster

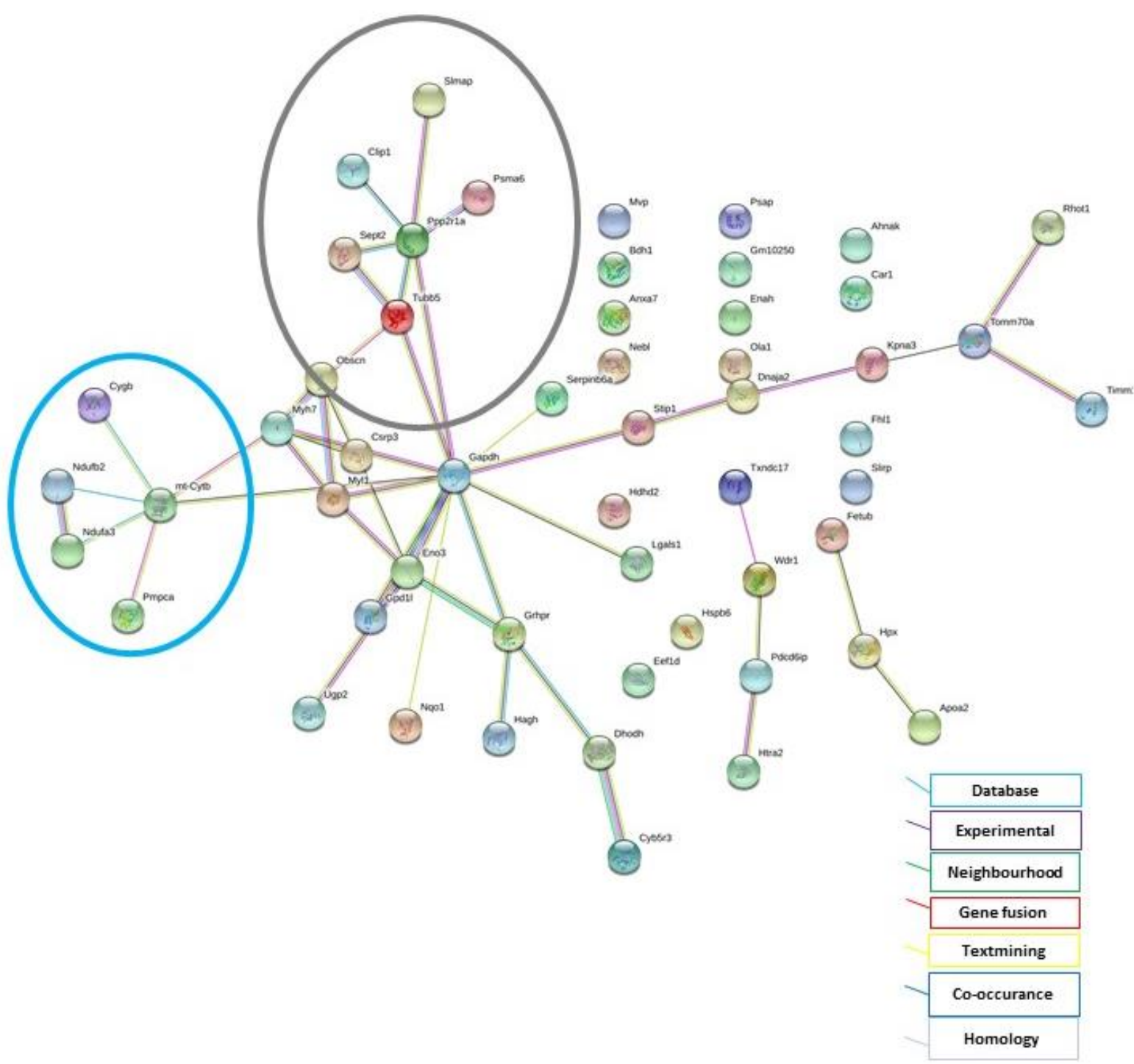

Fig. S2. Full view of STRING analysis of Group B, WT-SHAM vs WT-TAC proteome (up-regulated). Analysis of protein interaction network by STRING, Coloured lines between the proteins indicate the protein co-occurrence, co-expression, known interactions and predicted interactions. Up-regulated proteins show interconnecting mitochondrial proteins (blue circle) represents a functionally associated protein cluster, while cytoskeleton associated proteins are showing another cluster (grey circle) in up-regulated proteins. 


\section{Supplementary figure. S3: STRING cluster analysis}

WT-SHAM vs WT-TAC down-regulated protein cluster

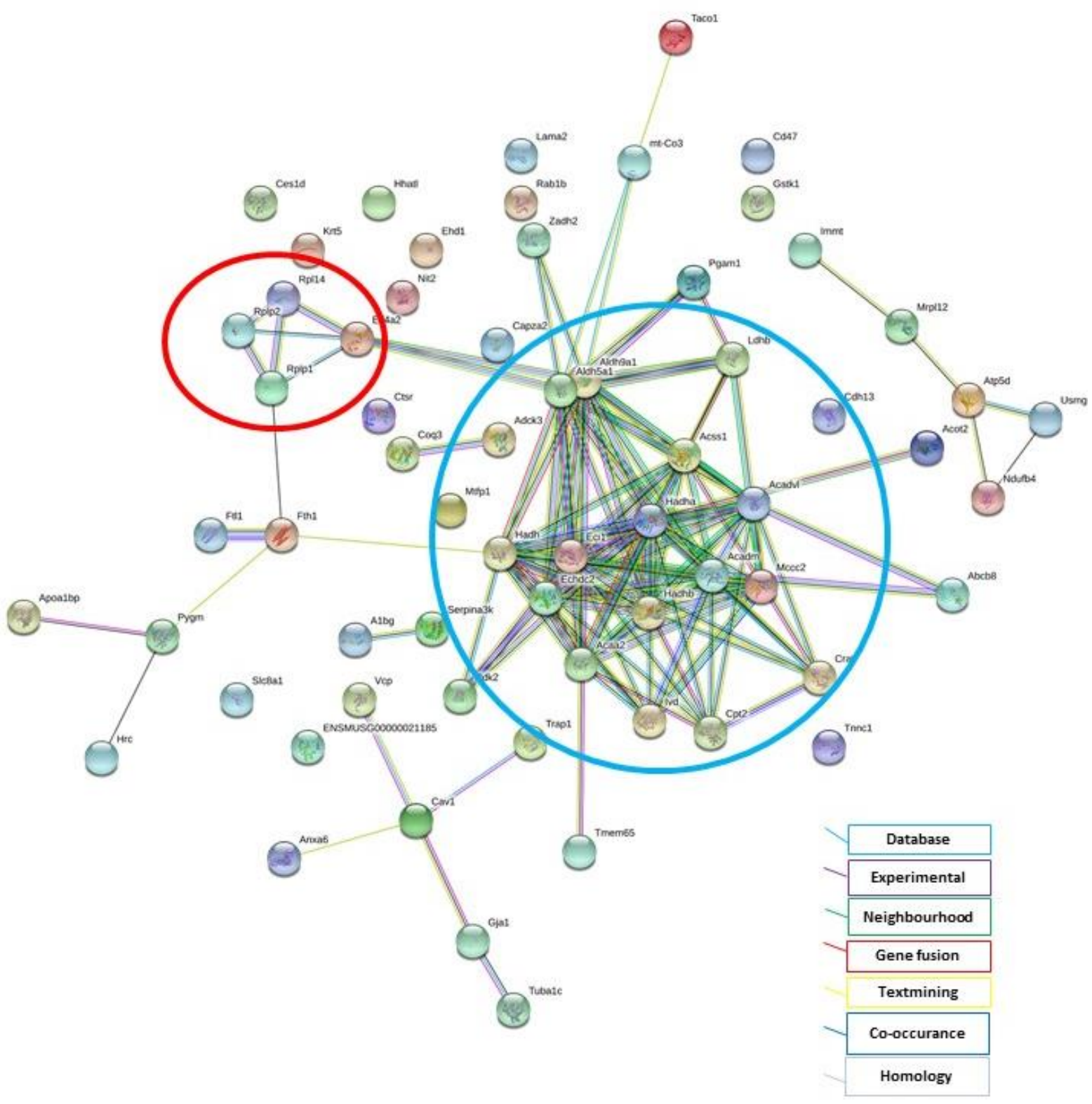

Fig. S3. Full view of STRING analysis of Group B, WT-SHAM vs WT-TAC proteome (down-regulated). Analysis of protein interaction network by STRING, Coloured lines between the proteins indicate the protein co-occurrence, coexpression, known interactions and predicted interactions. Interconnecting mitochondrial proteins (blue circle) represents a functionally associated protein cluster, while ribosomal associated proteins are showing another cluster (red circle) in downregulated proteins. 


\section{Supplementary figure. S4: STRING cluster analysis}

KO-SHAM vs KO-TAC up-regulated protein cluster

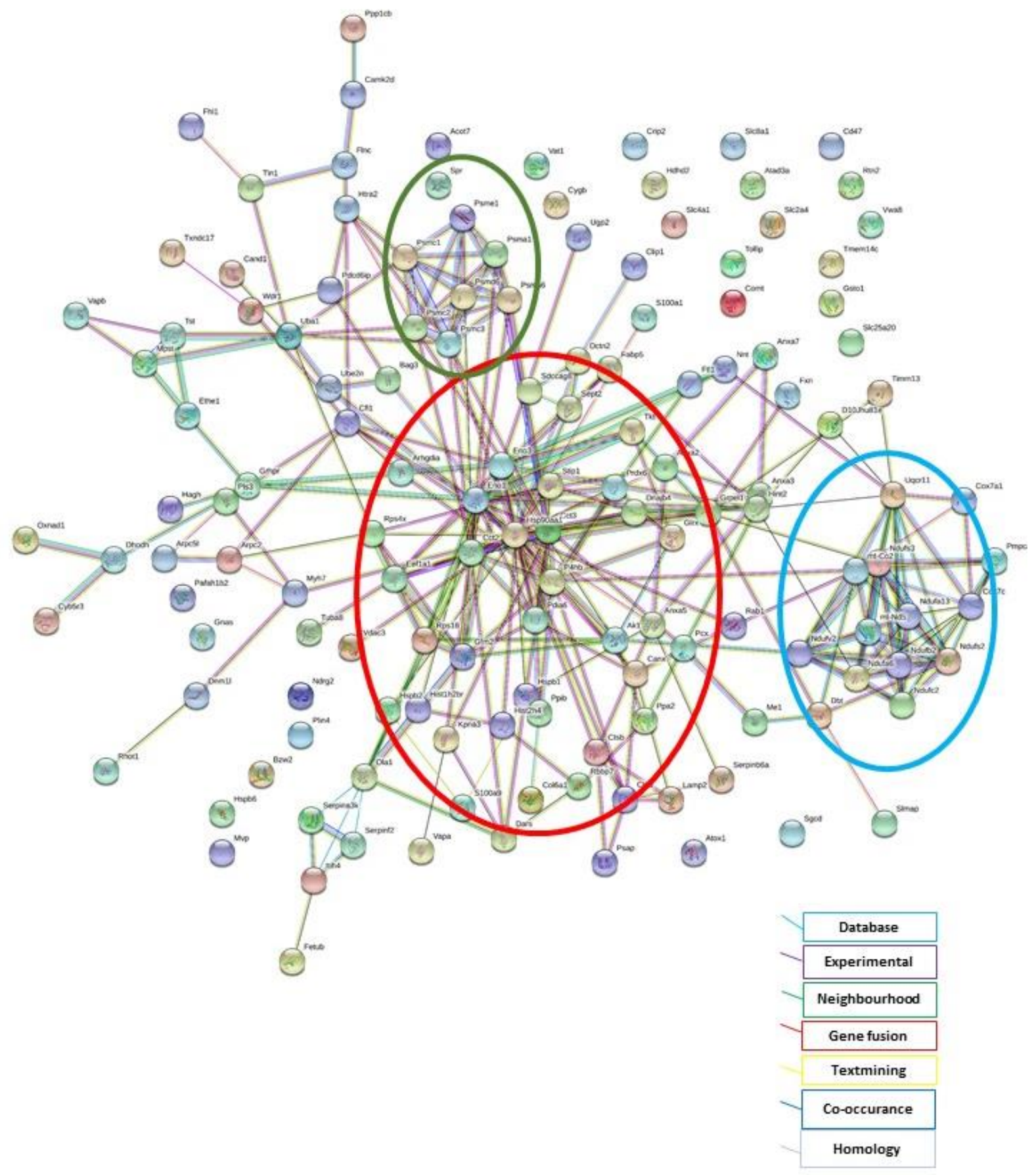

Fig. S4. Full view of STRING analysis of Group C, KO-SHAM vs KO-TAC proteome (up-regulated). Analysis of protein interaction network by STRING, Coloured lines between the proteins indicate the protein co-occurrence, co-expression, known interactions and predicted interactions. Up-regulated proteins show interconnecting major cluster (red circle) represents multiple enzymes involved in metabolic pathways and cytoskeleton associated proteins while, mitochondrial proteins (blue circle) represents a functionally associated protein cluster, while proteasome associated proteins are showing another cluster (green circle). 


\section{Supplementary figure. S5. STRING cluster analysis}

KO-SHAM vs KO-TAC down-regulated protein cluster

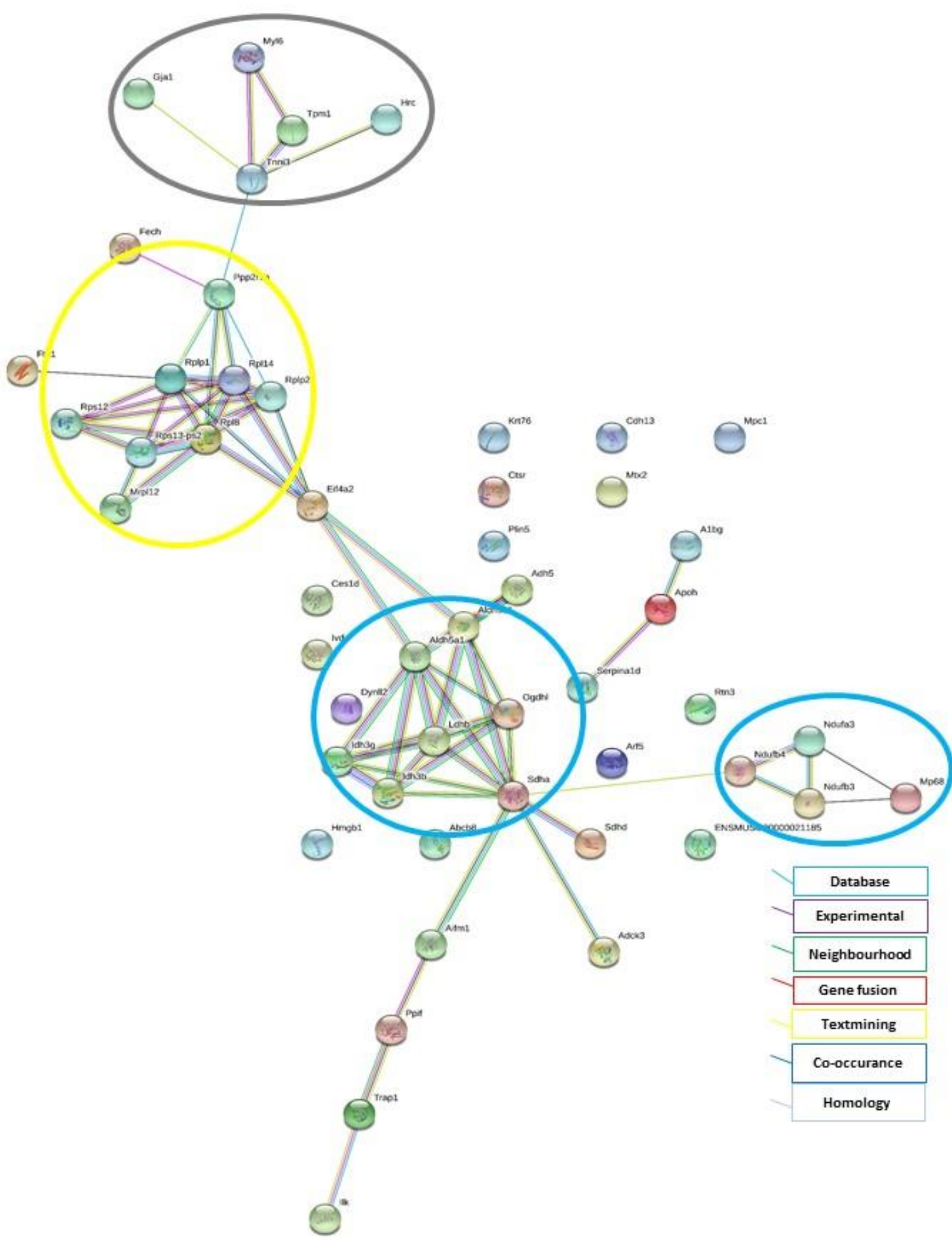

Fig. S5. Full view of STRING analysis of Group C, KO-SHAM vs KO-TAC proteome (down-regulated). Analysis of protein interaction network by STRING, Coloured lines between the proteins indicate the protein co-occurrence, coexpression, known interactions and predicted interactions. Interconnecting major clusters show mitochondrial proteins (blue circle), ribosomal proteins (yellow circle) and cytoskeleton proteins (grey circle) in down-regulated proteins. 


\section{Supplementary figure. S6: STRING cluster analysis}

WT-TAC vs KO-TAC up-regulated protein cluster

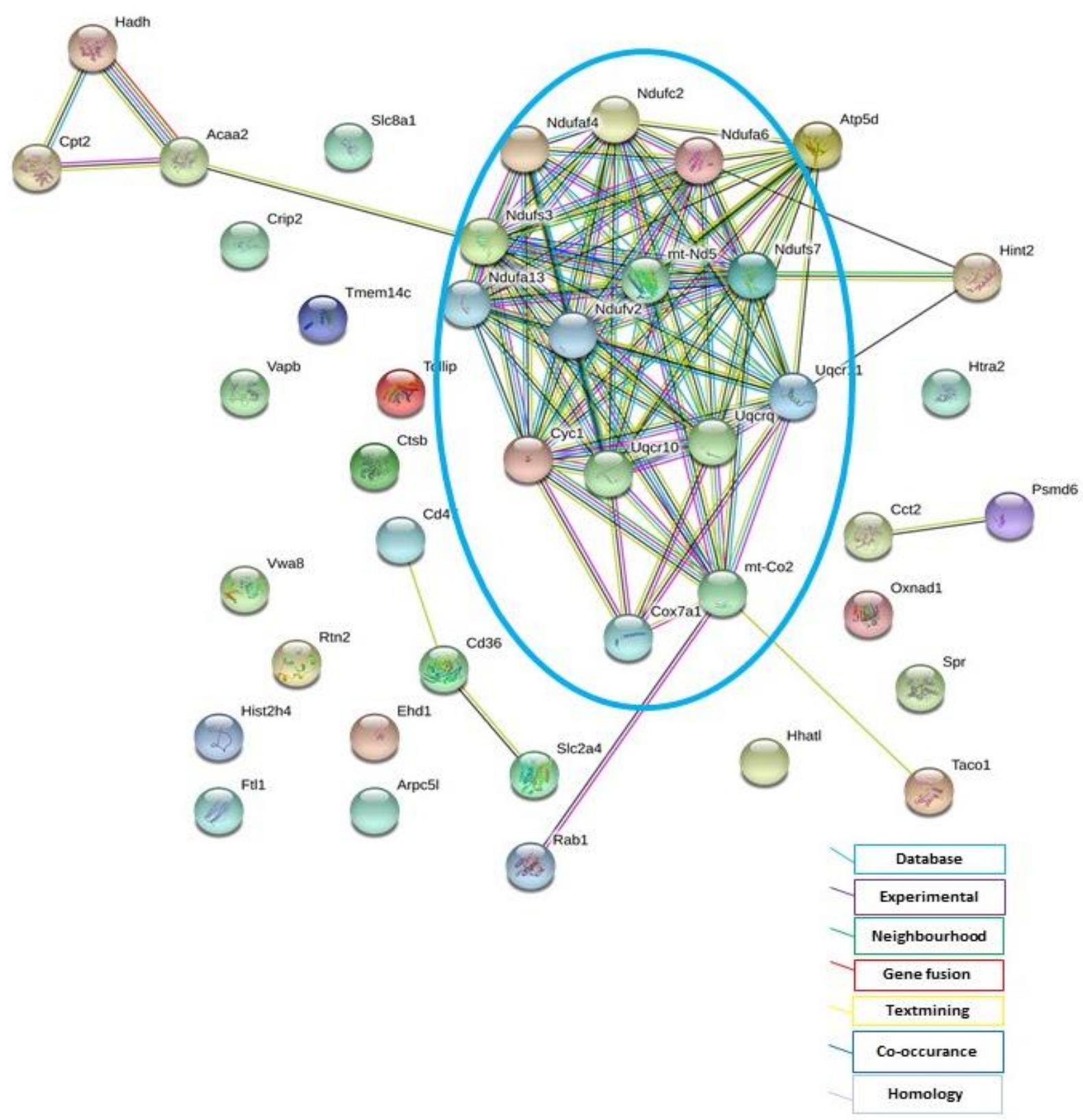

Fig. S6. Full view of STRING analysis of Group D, WT-TAC vs KO-TAC proteome (up-regulated). Analysis of protein interaction network by STRING, Coloured lines between the proteins indicate the protein co-occurrence, coexpression, known interactions and predicted interactions. Up-regulated proteins show Interconnecting major cluster shows mitochondrial proteins (blue circle). Interconnecting major clusters show ribosomal proteins (yellow circle) and cytoskeleton proteins (grey circle) (D). 


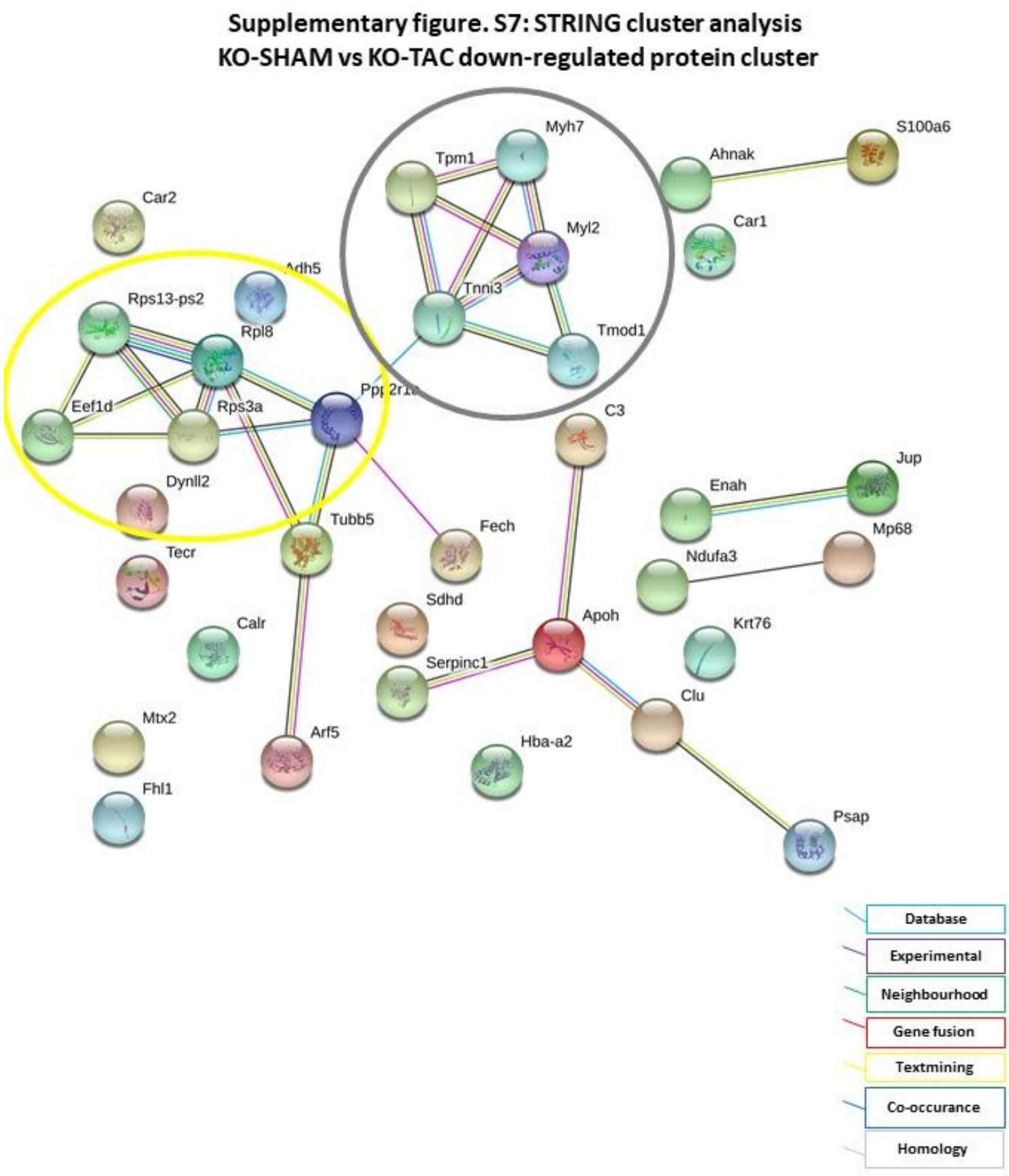

Fig. S7. Full view of STRING analysis of Group D, WT-TAC vs KO-TAC proteome (down-regulated). Analysis of protein interaction network by STRING, Coloured lines between the proteins indicate the protein co-occurrence, coexpression, known interactions and predicted interactions. Interconnecting major clusters show ribosomal proteins (yellow circle) and cytoskeleton proteins (grey circle). 


\section{Acknowledgements}

Ph.D. duration was one of the challenging times of my life, there are many people who helped me and made my journey feasible. There are not enough words to say thanks them for their help, love and guidance.

Firstly, I would like to express my deepest gratitude to my mentor Dr. Krishna Pantakani for providing me great opportunity to work with him. I would like to thank for his valuable support, guidance and for being always available to help me out not only in research work but in all other situations. I would like to thank him for his patience, enthusiasm and valuable scientific discussion and ideas for my Ph.D. work and projects. I also wanted to show my gratitude to him for guiding, editing and proof reading my annual reports, presentations, and without him this thesis too, would not have been completed. I consider myself very fortunate for being able to work with a very encouraging person.

I would like to sincerely thank Prof. Dr. Ibrahim Adham for accepting to be my first supervisor and member of my thesis committee. I would like to show my gratitude to his suggestions and guidance during my annual meetings and also for giving consent to edit my thesis.

I also owe gratitude to Prof. Dr. Sigrid Hoyer-Fender for being a part of my Thesis advisory committee and to be my co-referee. I want to thank her for providing guidance and support during my annual thesis committee meetings.

I would like to thank Prof. Dr. Abdul. R. Asif for providing an inspiring working environment in his research group at Institute for Clinical Chemistry for my Ph.D. studies. His valuable suggestions and support also guided me through difficult times of Ph.D. I would also like to thank him for proof reading my thesis despite his busy schedule.

I want to thank my institutional head Prof. Dr. Lutz Binder for allowing me to proceed my research work in Institute for Clinical Chemistry. I also want to thank him for travel grants and research funding whenever it was needed.

In my daily working environment, I have been blessed with a nice lab group. In my lab group firstly, I would like to thank Marlena Pantakani for scientific discussions and good friendship. She has been very helpful in optimization and troubleshooting of lab 
problems. Her calm and friendly nature also helped me in solving problems outside the work environment. I want to thank her for good time spent together.

I am indeed thankful to Reiner Andag, for his professional technical support and for being a great person to work with. I thank him for being so patient and helpful to multiple technical problems I would bring to him.

I also want to thank my other colleagues including Priyadharsini Raju, Rieke Welzbecher, Sandra Hartung, Ulrike and Jenny Gao for their support and help in lab. Most specifically, I want to thank my best friend Neelam Younas for her care, kindness, fantastic positive attitude to work and tremendous moral support in my difficult times. I want to thank her for our shared laughter, fights, late night walks, travel and drama. She has been an excellent listener and good advisor throughout these years. There are no words to explain how much she helped and guided me.

I also want to show my gratitude to Dr. Muzna Zahur for her friendship, care and support. Her nice and positive attitude always helped me to pass through difficult times.

I would like to thank my other friends from all over the world for their support and encouragement. Special thanks to Sehar Afreen (for long telephonic chit chat), Anum Ali Ahmad (for being positive and supportive), Humaira Gul (for shared laughter and encouragement) and Madiha Nasir (for lively discussions). I also want to thank Maryam Afzal (for humour and irritating me with her singing talent), Nida Amin (for being supportive) Atika Wahab (for being a nice company), and Mohsin Shafiq (for helping $\mathrm{me})$.

Last but not least I owe huge gratitude to my family members for their love, support, prayer and trust. This thesis for sure is a tribute to my Late Father "Shahid Bashir" for giving me strength and giving me confidence to achieve my goals. I want to thank my mother "Naveeda Shahid", but what can I say? There are no words to describe her love, care and courage. It wouldn't have been possible for me to complete my Ph.D. without your support and your faith in me.

Most specifically, I want to thank my eldest sister Mariam Shahid for being so supportive in my life. Her care, advise, love, scolding and continued moral support gave me strength to be here today. I want to thank her for being a role model for me with her bravery, courage and strength. I am indebted to her for giving a greatest gift of my life 
in September 2009 which brought brightness, happiness and purpose in my life. I want to show my gratitude to my sisters (Faiza, Humera, Sadia, Rabia, Binte-Azra and Fehda) and to my brothers (Farooq, Zubair, Faisal, Khurram, Imran, Talha and Ahsan) for their love, prayers and support. During my Ph.D. times, I missed you guys a lot, but it also gave me realisation that I have been blessed with a great, sweet and supportive family.

My acknowledgements are incomplete without special thanks to my little sweet stars Moeen, Hadiya, Umama, Mohid, Hafsa and Khizar. Without any doubt I missed you guys the most and your videos and pictures gave me happiness and a lift whenever I was sad. You guys made my visits memorable and cherishable.

At the end I would like to thank to Higher education commission Pakistan (HEC) and German Academic Exchange Service (DAAD) for funding my Ph.D. 


\section{Curriculum Vitae}

Name

Address

Email

Date of birth

Place of birth

Permanent address

Nationality

Languages

Sidra Shahid

Herman Rein Strasse 9, Apartment No. 314, 37075, Goettingen

shahidsidra@yahoo.com

21.05.1987

Lahore, Pakistan

109, Cll block, Johar Town Lahore, Pakistan

Pakistan

English, Urdu, German

\section{Educational Background}

2014 to date $\quad$ Ph.D. student, Dissertation: "Role of BRD4 and its target Ptp1b in Endothelial cells and in cardiovascular disease models"

Institute for Clinical Chemistry, UMG, Goettingen.

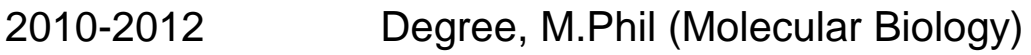

Dissertation: "Linkage analysis is a tool for carrier screening and genetic counselling of hearing impairment."

Centre of excellence in Molecular Biology (CEMB)

University of Punjab, Lahore Pakistan.

2007-2009 Degree, M.Sc (Microbiology and Molecular Genetics)

Department of Microbiology and Molecular Genetics (MMG)

University of Punjab, Lahore Pakistan.

2005-2007

Degree, B.Sc (Biology)

University of Punjab, Lahore Pakistan.

2003-2005

Degree F.Sc (pre-Medical)

Lahore board Pakistan. 


\section{Oral Presentation}

123. German Conference of Laboratory Medicine Mannheim, 30 $0^{\text {th }}$ September 2016. Title: "Phosphoproteome analysis of mouse hearts from endothelial specific knockout of Protein Tyrosine phosphatase 1B (PTP1B)". Abstract published online in "Clinical Chemistry and Laboratory Medicine (CCLM)", Volume 54, Issue 10, Pages eA367-eA473, ISSN (Online) 1437-4331, ISSN (Print) 1434-662".

\section{Poster Presentation}

125. German Conference of Laboratory Medicine Mannheim, 29 ${ }^{\text {th }}$ September 2018. Title: "Inhibition of BRD4 by JQ1 and RVX-208 rescues endothelial cell dysfunction in an in vitro model of atherosclerosis". Abstract will be published online in "Clinical Chemistry and Laboratory Medicine (CCLM), Volume 57. 